\title{
Indoor physical field estimation from sparse sensor observations
}

\section{JIANG CHAOYANG}

\section{School of Electrical and Electronic Engineering}

\author{
A thesis submitted to the Nanyang Technological University \\ in partial fulfillment of the requirements for the degree of \\ Doctor of Philosophy
}





\section{$\underline{\text { Statement of Originality }}$}

I hereby certify that the intellectual content of this thesis is the product of my original research work and has not been submitted for a higher degree to any other University or Institution. 



\section{Acknowledgements}

I would like to express my deepest gratitude to my advisor, Professor Soh Yeng Chai, for his professional guidance on my research works. He offered me valuable insights on the selection of research topics. He took time to improve my academic writing skills. He trained my independence on conducting research works. I want to express my thanks to him for his full academic support, his financial support, and his trust and encouragement. I feel extremely blessed under the supervision of Prof. Soh. I learned a lot from him, not only on projects, but also scientific attitudes, problem-solving skills, and presentation skills. I also wish to give my greatest gratitude to my co-advisor, Associate Professor Li Hua, for his support on CFD simulations. He guided me on choosing research directions and paper publications. I also wish to thank him for the suggestions and help on the experiments, the discussions and ideas, his support on job-seeking, and his trust and encouragement.

I would like to sincerely thank my group mate, Mustafa Khalid Masood, for his contributions to data collection and his help on polishing papers. I want to thank Zhu Qingchang for sharing the conference trip. I wish to thank Zhai Deqing for his help on the experiments. I also want to thank Dr. Lin Chen for his help on CFD simulation. Thanks to Zhou Xiaoli, Ji Ke, Zhang Xingyu, and Huynh Nam Khoa for their help on data collection. I would also like to thank another two group mates, Xu Jinming and Chen Zhenghua, for their help and pieces of valuable advice.

I would like to thank my previous office mate, Dr. Yu Hang, for his help on using SDPT3 and exchanging academic perspectives. I want to thank my current office mate, Wei Zhe. I shared almost every day with him my last three years Ph.D study: lunch time, coffee breaks, and sports time. Thanks to him for his precessional expertise on image editing. I also want to thank other office mates, Zhao Wei, Guan Zheming, Wang Mengmeng, Wang Hao, and Zhang Shuai, who made me feel comfort in our office. 
I would like to thank my wife, Zhao Yang, for her support, for her love, for everything she did for me. The marriage of us was the happiest moment during the four years Ph.D candidature. She motivated me to do better every day.

I want to take this chance to thank my parents for their support and endless unconditional love. Thanks to my two brothers for their help and their care to our parents. Great thanks to my parents-in-law for their understanding, support and suggestions. 


\section{Abstract}

Indoor physical fields (e.g. thermal map, pressure field, air velocity field, etc.) are very useful information for energy efficient air-conditioning system design. Computational fluid dynamics (CFD) simulation is the most popular tool to estimate indoor physical fields. But the simulation is computationally expensive and timeconsuming. The simulation results are also inaccurate compared with sensor observations. Therefore, a new method to rapidly find the accurate estimation of indoor physical fields is very interesting.

In this work, we show how sparse sensor observations can be effectively used for indoor physical fields estimation by solving the three research problems:

1. calibration of simulated physical fields using sparse sensor observations;

2. reconstruction of physical fields from sparse sensor observations;

3. sensor placement for the optimal physical field reconstruction.

Current CFD calibration work has mainly focused on the calibration of input parameters of CFD simulation. No reported publication has considered the calibration of the simulation results. We took inspiration from the image editing problem and developed a methodology to calibrate the simulated physical fields based on sparse sensor observations. We formulated the calibration work to be an optimization problem. The cost function consists of two terms. One term guarantees a good local adjustment of the simulated physical fields. The other term transmits the adjustment from local regions around sensing locations to the global domain. The proposed method can enhance the simulated physical field while preserving the overall original profile. An experiment was implemented in an air-conditioned room to demonstrate the feasibility of the method. Four sensor observations were used to calibrate a simulated thermal map with 167x365 data points. The experimental results show the effectiveness of the proposed method. 
We then provided a two-stage physical field reconstruction approach to rapidly reconstruct a physical field from the observed input parameters of the CFD simulation and sparse observations of the physical field. With a physical field database obtained from CFD simulations, we can find the principal component analysis (PCA) modes of the physical fields. In the first stage, we built a regression model between the input parameters and the PCA coefficients. To find this model, we provided a scaled extreme learning machine (sELM) algorithm. With the regression model, we can obtain the PCA coefficients and reconstruct an approximated physical field. In the second stage, we estimated the error of the approximated physical field from sparse sensor observations, with which we can correct the physical field estimated in the first stage. The proposed method is shown better than current methods even using fewer sensor observations.

With the above results, we noted that sensor placement is a critical problem for physical field reconstruction. To address the problem, we develop a new greedy algorithm, named maximal projection on minimum eigenspace (MPME). In this algorithm, we select the sensing locations one-by-one. The least number of required sensors can be determined by checking whether the estimation accuracy is satisfied after each sensing location is determined. The minimum eigenspace is defined as the eigenspace associated with the minimum eigenvalue of the dual observation matrix. For each sensing location, the projection of its observation vector onto the minimum eigenspace is shown to be monotonically decreasing w.r.t. the worst case error variance (WCEV) of the estimated parameters. We select the sensing location whose observation vector has the maximum projection onto the minimum eigenspace of the current dual observation matrix. The proposed MPME is shown to be one of the most computationally efficient algorithms. Our Monte-Carlo simulations show that MPME outperforms the state of the art, especially when the number of available sensors is very limited. The MPME algorithm can be easily used for the indoor physical field reconstruction, but the current methods cannot because of their high storage requirement and high computation cost.

Keywords: Sensor networks, computational fluid dynamics, physical field calibration, physical field reconstruction, sensor placement, image editing, principal component analysis, scaled extreme learning machine, inverse problem, greedy algorithm. 


\section{Contents}

Acknowledgements $\quad$ i

Abstract iii

List of Figures $\quad$ ix

List of Tables $\quad$ xiii

Symbols and Acronyms $\quad$ xv

1 Introduction 1

1.1 Background . . . . . . . . . . . . . . . . . 1

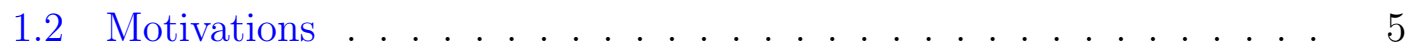

1.3 Literature review . . . . . . . . . . . . . . . 8

1.3.1 Calibration of the simulated physical field with sparse sensor observations . . . . . . . . . . . . . . 8

1.3.2 Physical field reconstruction from sparse sensor observations 10

1.3.3 Sensor placement for the optimal physical field reconstruction 12

1.4 Methodology . . . . . . . . . . . . . . . . . . . 14

1.5 Outline and contributions . . . . . . . . . . . . . 19

2 Calibration of simulated physical fields 23

2.1 Problem formulation . . . . . . . . . . . . . . . . . . 24

2.2 The proposed methodology . . . . . . . . . . . . . 25

2.3 Numerical method to solve the optimization problem . . . . . . . . 27

2.4 Computational efficient approximated solution _. . . . . . . . . 30

2.5 Experimental verification: indoor thermal map calibration . . . . . 32

2.5.1 Statement of the experiment . . . . . . . . . . . . . . . . . 32

2.5.2 CFD simulation . . . . . . . . . . . . . 33

2.5.3 The calibration results . . . . . . . . . . . . . . . . . . . . . . . . . 37

2.5.4 The influence of balance factor . . . . . . . . . . . . . . 39

2.5.5 The influence of magnitude variance . . . . . . . . . . . . 44

2.5.6 The influence of distance variance . . . . . . . . . . . . . 46

2.6 Concluding remarks . . . . . . . . . . . . . . . . . . . . . 48 
3 Physical field reconstruction $\quad 51$

3.1 Problem Formulation . . . . . . . . . . . . . . . . . . . 52

3.2 PCA for physical field reconstruction . . . . . . . . . . . . . . 54

3.2.1 PCA modes of the physical fields . . . . . . . . . . . 54

3.2.2 PCA coefficients estimation from the observed input parameters ........................ 57

3.2.3 Dominant PCA coefficients estimation from sparse observations 60

3.3 Two-stage physical field reconstruction . . . . . . . . . . . . . . 61

3.3.1 First-stage: physical field reconstruction from observed input parameters ....................... 62

3.3.1.1 Standard ELM for physical field reconstruction . . 62

3.3.1.2 Scaled ELM for physical field reconstruction . . . . 64

3.3.2 Second-stage: physical field enhancement based on the sparse sensor observations . . . . . . . . . . . . . . . . 68

3.4 Error analysis . . . . . . . . . . . . . . . . . . . 70

3.4.1 Error of the physical field estimated from the observed input parameters ...................... 70

3.4.2 Error of the physical field estimated from sparse sensor observations . . . . . . . . . . . . . . . 71

3.4.3 Error of the physical field estimated via TSPFR . . . . . . 72

3.4.4 Error comparison . . . . . . . . . . . . . . . . 73

3.5 Sensor placement . . . . . . . . . . . . . . . . 75

3.6 Number reduction of the sensor observations . . . . . . . . . . . . 76

3.7 Example: indoor thermal map reconstruction . . . . . . . . . . . . 79

3.7.1 Thermal map database . . . . . . . . . . . . . . . . . 80

3.7.2 The PCA modes . . . . . . . . . . . . . . . . . 82

3.7.3 Results and discussions . . . . . . . . . . . . . . . 83

3.7.4 Number reduction of the sensor observations . . . . . . . . . 91

3.8 Concluding remarks . . . . . . . . . . . . . . . . . . . . 95

4 Sensor placement for the optimal physical field reconstruction $\quad \mathbf{9 7}$

4.1 Problem formulation . . . . . . . . . . . . . . . . . . . . . . . . . 98

4.1.1 Physical field reconstruction . . . . . . . . . . . . . . . . . . . . . . . . 98

4.1 .2 Sensor placement . . . . . . . . . . . . . . 100

4.2 The state of the art . . . . . . . . . . . . . . . . . . . . 101

4.2.1 Convex relaxation . . . . . . . . . . . . . . . 101

4.2 SparSenSe . . . . . . . . . . . . . . . . . 102

4.2 .3 FrameSense . . . . . . . . . . . . . . . . . 102

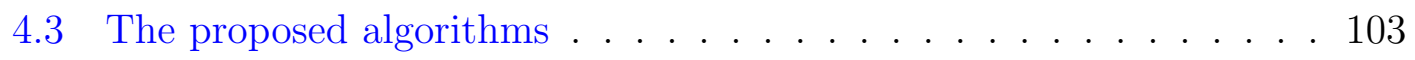

4.3.1 Minimum nonzero eigenvalue pursuit (MNEP) . . . . . . . 106

4.3.2 Maximal projection on minimum eigenspace (MPME) . . . . 109

4.3.3 Discussions about MNEP and MPME . . . . . . . . . . 115

4.4 Effectiveness of the MPME algorithm . . . . . . . . . . . . . 116

4.4.1 Comparison with convex relaxation . . . . . . . . . 120 
4.4.2 Comparison with SparSenSe . . . . . . . . . . . . . 121

4.4.3 Comparison with FrameSense . . . . . . . . . . . . . 123

4.4 Local optimization . . . . . . . . . . . . . . . . . 127

4.5 Computational cost of the MPME algorithm . . . . . . . . . . . . . 129

4.6 Storage requirement of the MPME algorithm . . . . . . . . . . . . 130

4.7 Concluding remarks . . . . . . . . . . . . . . . . 132

5 Conclusions and future work $\quad 135$

$\begin{array}{ll}\text { A Proof of Theorem 2 } & 141\end{array}$

B Proof of Theorem $3 \quad 143$

$\begin{array}{ll}\text { C Proof of Theorem } 4 & 145\end{array}$

$\begin{array}{ll}\text { Author's Publications } & 151\end{array}$

$\begin{array}{lc}\text { Bibliography } & 153\end{array}$ 



\section{List of Figures}

1.1 The temperature field (at degree centigrade) of the symmetric plane of Lecture Theatre 22 at Nanyang Technological University, Singapore. 3

1.2 The velocity streamline of an office room obtained from CFD simu-

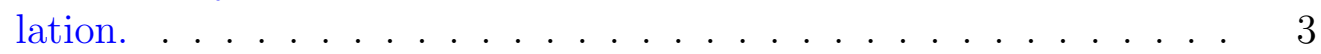

1.3 An simple example of image editing. The dots in the left image are used as control samples to edit the input image. . . . . . . . . . 15

1.4 The block diagram of the two-stage physical field reconstruction . . 16

2.1 Illustration of the low rank approximation of $W_{s}$. . . . . . . . . 31

2.2 The test bed: a $7.28 \mathrm{~m} \times 3.32 \mathrm{~m} \times 2.5 \mathrm{~m}$ room located at the Process Control and Instrumentation Lab in Nanyang Technological University, Singapore. The bottom figure is the top view sketch map. The red square and the red rectangle are the two outlets. The center one is for induced air, and the corner one is for exhaust air. The two blue rectangles represent the two cooling air inlets. The black triangle represents an air velocity sensor, as shown in Figure 2.3(A). The black dot represents a temperature sensor as shown in Figure 2.3(B). The four red stars and four red dots represent eight temperature sensors located $1.2 \mathrm{~m}$ above the ground. The four black squares are four heaters. The hight of the heaters were $1 \mathrm{~m}$ above the ground. 34

2.3 Sensors used for indoor parameters measurement. (A): velocity sensor used to measure the inlet air velocity. (B): temperature sensor used to measure the inlet cooling air temperature. (C): temperature sensor used to measure the temperature at $1.2 \mathrm{~m}$ hight, which is hung

2.4 The recorded inlet cooling air velocity and temperature during 11:17 to $13: 10$ on 26th May, 2016. . . . . . . . . . . . . . . 36

2.5 The temperature recording of the seven sensors (s1-s7) . . . . . . 37

2.6 Convergence history of the residuals. . . . . . . . . . . . . . 38

2.7 The thermal map (temperature field in ${ }^{\circ} \mathrm{C}$ ) $1.2 \mathrm{~m}$ above the ground. $\quad 39$

2.8 The estimated error $(\hat{\mathbf{v}})$ of the thermal map in Figure 2.7(A) w.r.t. different balance factor. $\sigma_{m}=1000$ and $\sigma_{d}=1$. . . . . . . . . 40

2.9 The errors of CFD solution and the calibrated thermal maps at the sensing locations s1-s8 with $\sigma_{m}=1000$ and $\sigma_{d}=1 \ldots 41$

2.10 The estimated error $(\hat{\mathbf{v}})$ of the thermal map in Figure 2.7(A) w.r.t. different magnitude variance $\sigma_{m} . \alpha=0.01$ and $\sigma_{d}=1 . \ldots . . .443$ 
2.11 The errors of CFD simulation result and the calibrated thermal maps at the sensing locations s1-s8 with $\alpha=0.01$ and $\sigma_{d}=1 \ldots 44$

2.12 The estimated error $(\hat{\mathbf{v}})$ of the thermal map in Figure 2.7(A) w.r.t. different distance variance $\sigma_{d} . \alpha=0.01$ and $\sigma_{m}=1000 \ldots \ldots$. . .

2.13 The errors of CFD simulation result and the calibrated thermal maps at the sensing locations s1-s8 with $\alpha=0.01$ and $\sigma_{m}=1000 \ldots 48$

3.1 The block diagram of the two-stage physical field reconstruction . . 61

3.2 The structure of the standard extreme learning machine (ELM). . . 63

3.3 The sigmoid function. . . . . . . . . . . . . . . . . . 65

3.4 Top view sketch map of the room and the four heaters. . . . . . . . 80

3.5 Percentage energy of the PCA modes . . . . . . . . . . . . . . 82

3.6 The ground truth $\left({ }^{\circ} \mathrm{C}\right)$ : the thermal map $1.2 \mathrm{~m}$ above the ground of the thermal lab in Figure 2.2, which is obtained from the CFD simulation. . . . . . . . . . . . . . .

3.7 The estimated thermal map $\left({ }^{\circ} \mathrm{C}\right)$ : spline interpolation technique is used to estimate the PCA coefficients from the observed input parameters $\mathbf{p}_{\mathrm{o}} \ldots \ldots \ldots \ldots \ldots \ldots$

3.8 The estimated thermal map $\left({ }^{\circ} \mathrm{C}\right)$ : the standard ELM is used to estimate the PCA coefficients from the observed input parameters $\mathbf{p}_{\mathrm{o}}$. Both the random weights and the random bias follow the normal distribution $\mathcal{N}\left(0,0.00025^{2}\right)$. The number of hidden neurons $L=$ 100 , and the regularization parameter $\gamma=0.00001 . \ldots . . .$.

3.9 The estimated thermal map $\left({ }^{\circ} \mathrm{C}\right)$ : the sELM is used to estimate the PCA coefficients from the observed input parameters $\mathbf{p}_{\mathrm{o}}$. Both the random weights and the random bias follow the normal distribution $\mathcal{N}\left(0,0.025^{2}\right)$. The number of hidden neurons $L=100$, and the regularization parameter $\gamma=0.00001 \ldots \ldots \ldots \ldots$

3.10 The estimated thermal map $\left({ }^{\circ} \mathrm{C}\right)$ : the least square technique is used to estimate the dominant PCA coefficients from the four sensor observations. The stars represent the sensing locations.

3.11 The estimated thermal map $\left({ }^{\circ} \mathrm{C}\right)$ : the TSPFR method is used to estimate the PCA coefficients from both the observed input parameters $\mathbf{p}_{\mathrm{o}}$, and the four sensor observations. In the first stage, we use the spline interpolation method to determine the regression model. The stars represent the sensing locations. . . . . . . . . . . .

3.12 The estimated thermal map $\left({ }^{\circ} \mathrm{C}\right)$ : the TSPFR method is used to estimate the PCA coefficients from both the observed input parameters $\mathbf{p}_{\mathrm{o}}$, and the four sensor observations. In the first stage, we use sELM to train the regression model. The stars represent the sensing locations. . . . . . . . . . . . . . . .

3.13 The error of estimated thermal maps. The PCA coefficients are estimated from the observed input parameters $\mathbf{p}_{\mathrm{o}}$ using three different regression models. . . . . . . . . . . . . . 
3.14 The error of the estimated thermal map in Figure $3.10\left({ }^{\circ} \mathrm{C}\right)$ : the least square technique is used to estimate the dominant PCA coefficients from the four sensor observations. . . . . . . . . . . . . .

3.15 The error of the estimated thermal maps. The TSPFR method is used to estimate the PCA coefficients from both the observed input parameters $\mathbf{p}_{\mathrm{o}}$ and the four sensor observations. . . . . . . .

3.16 The error of two estimated thermal maps. The TSPFR method is used to estimate the thermal map from the observed input parameters $\mathbf{p}_{\mathrm{o}}$ and TWO sensor observations. Only the first and the fourth PCA modes are used in the second stage. . . . . . . . . . .

4.1 The performance comparison between the MPME and other sensor placement methods for Example 1. For the MPME and the MNEP we do not consider the stopping criteria but show the mean WCEV index and the mean MSE index w.r.t. the number of sensor nodes, increasing from 20 to 40. For the SparSenSe method, we do not consider the minimum number of required sensor nodes but determine the sensing locations like the convex relaxation method by finding the indices of the $M$ largest elements of $\mathbf{w}^{*}$. . . . . . . . . . .

4.2 The performance comparison between the MPME and other sensor placement methods for Example 2. For the MPME and the MNEP we do not consider the stopping criteria but show the mean WCEV index and the mean MSE index w.r.t. the number of sensor nodes, increasing from 20 to 40. For the SparSenSe method, we do not consider the minimum number of required sensor nodes but determine the sensing locations like the convex relaxation method by finding the indices of the $M$ largest elements of $\mathbf{w}^{*}$. . . . . . . . . . . . 119

4.3 The mean condition number of the dual observation matrix $\boldsymbol{\Psi}_{k}=$ $\boldsymbol{\Phi}_{k}^{\mathrm{T}} \boldsymbol{\Phi}_{k}$ for Example 1. Here $\boldsymbol{\Phi}_{k}$ is obtained from the five sensor placement algorithms. . . . . . . . . . . . . . . . . 121

4.4 Selected sensing indices with SparSenSe for Example 3. . . . . . . . 122

4.5 The MSE index of $\hat{\boldsymbol{\alpha}}$ estimated from 20 to 40 sensor observations for Example 3. . . . . . . . . . . . . . . . . . . . . . . . 123

4.6 The mean WCEV index and mean MSE index of $\hat{\boldsymbol{\alpha}}$ estimated from 20 to 40 sensor observations for Example 4. . . . . . . . . . . . . 125

4.7 The performance comparison between MPME and five methods with local optimization for Example 1 . . . . . . . . . . . . . 126

4.8 The performance comparison between MPME and five methods with local optimization for Example 4. . . . . . . . . . . . . . 127 
4.9 The mean computation time of the five sensor placement algorithms for $N \times 20$ Gaussian random matrices where $N$ is from 100 to 1000 . In the simulations, the number of available sensor nodes, i.e. $M$, is set as 20 for the convex relaxation method, FrameSenSe, MNEP and MPME. The computation time is estimated by the MATLAB tic-toc commands. The simulation is run in a laptop with a $2.4 \mathrm{GHz}$ Intel i3-3110M processor. The mean computation time is the mean value of 50 different simulations, and for each simulation, $\tilde{\boldsymbol{\Phi}} \in \mathbb{R}^{N \times 20}$ with independent entries $\varphi_{i j} \sim \mathcal{N}(0,1) \ldots \ldots . \ldots . \ldots . \ldots 130$

4.10 The estimated thermal map using the proposed TSPFR method. The stars represent the sensing locations obtained from the proposed MPME algorithm. The signal representation matrix $\tilde{\boldsymbol{\Phi}}$ is obtained from the domiannt PCA modes. . . . . . . . . . . . . . . . . . 131 


\section{List of Tables}

2.1 Boundary conditions of the CFD simulation . . . . . . . . . . . . 37

2.2 The RMSE of the thermal maps data at the sensing locations w.r.t. different balance factor . . . . . . . . . . . . . . . . . 42

2.3 The RMSE of the thermal maps data at the sensing locations w.r.t. different magnitude variance $\sigma_{m} \ldots \ldots$. . . . . . . . . . . 45

2.4 The RMSE of the thermal maps data at the sensing locations w.r.t. different distance variance $\sigma_{d} \ldots \ldots \ldots$. . . . . . . . . 46

3.1 Percentage energy and cumulative energy content for PCA modes . 82

3.2 The performance indices of the estimated thermal maps . . . . . . . 90

3.3 The mean absolute error (mAE) of the estimated PCA coefficients for leave-one out cross validation $(\mathrm{LOOCV})$. . . . . . . . . . . . 91

3.4 The performance indices of the estimated thermal maps when the number of sensor observations is smaller than the number of dominant PCA modes. . . . . . . . . . . . . . . . . 93

3.5 The error of the dominant PCA coefficients estimated from different methods. . . . . . . . . . . . . . . . . . . 93

4.1 The computational effort of the five sensor placement methods . . . 130 



\section{Symbols and Acronyms}

\section{Symbols}

$\mathbb{R}^{n}$

$\|\cdot\|$

$\langle\cdot, \cdot\rangle$

$\Delta$

0

1

$O(\cdot)$

$(\cdot)^{\dagger}$

$\mathbf{E}(\cdot)$

$\operatorname{tr}(\cdot)$

$\operatorname{det}(\cdot)$

$\operatorname{span}(\cdot)$

$\operatorname{null}(\cdot)$

$\operatorname{rank}(\cdot)$

$\mathcal{N}\left(0, \sigma^{2}\right)$ $\mathcal{B}(1, p)$ the $n$-dimensional Euclidean space

the norm of a vector or matrix in Euclidean space

the inner product of two vectors

the Laplace operator

all zero entry matrix with proper dimension

all one entry column vector with proper dimension

order of magnitude

the Moore-Penrose pseudo inverse

the expectation operator of one random variable

the trace operator

the determinant operator

the spanned space operator

the null space operator

the rank operator of a matrix

normal distribution with zero mean and $\sigma^{2}$ variance

Bernoulli distribution with success probability $p$

\section{Acronyms}

ACBs Active Chilled Beam system

CFD Computational Fluid Dynamics

ELM Extreme Learning Machine

FP Frame Potential

HVACs Heating, Ventilating and Air-Conditioning systems 


$\begin{array}{ll}\text { LOOCV } & \text { Leave One Out Cross Validation } \\ \text { MIMO } & \text { Multi-Input-Multi-Output } \\ \text { MISO } & \text { Multi-Input-Single-Output } \\ \text { MNEP } & \text { Minimum Nonzero Eigenvalue Pursuit } \\ \text { MPME } & \text { Maximal Projection on Minimum Eigenspace } \\ \text { mAE } & \text { mean Absolute Error } \\ \text { MAE } & \text { Maximum Absolute Error } \\ \text { MSE } & \text { Mean Squared Error } \\ \text { MVUE } & \text { Minimum Variance Unbiased Estimation } \\ \text { PCA } & \text { Principal Components Analysis } \\ \text { PDE } & \text { Partial Differential Equation } \\ \text { PIV } & \text { Particle Image Velocimetry } \\ \text { RMSE } & \text { Root Mean Square Error } \\ \text { SAE } & \text { Sum of Absolute Error } \\ \text { sELM } & \text { scaled Extreme Learning Machine } \\ \text { SLFNs } & \text { Single-hidden-Layer Feedforward Neural-networks } \\ \text { SparSenSe } & \text { Sparse-aware Sensor Selection } \\ \text { TSPFR } & \text { Two-Stage Physical Field Reconstruction } \\ \text { WCEV } & \text { Worst Case Error Variance }\end{array}$




\section{Chapter 1}

\section{Introduction}

\subsection{Background}

The global energy usage and demand is growing rapidly as nations pursue economic growth and infrastructure development. It has led to environmental problems such as climate change and global warming. Around one-third of the energy consumption is consumed in buildings, and is still climbing steadily [1]. According to the recently published data, a large part of building energy consumption is attributable to Heating, Ventilating and Air-Conditioning (HVAC) systems [2-4]. Without a doubt, the upward trend of energy consumption is continuing; therefore, improving the energy efficiency of HVAC systems is increasingly important.

A recent review paper [3] showed that we can enhance energy efficiency of HVAC systems via three main perspectives:

- novel cooling devices (e.g., energy efficient heat/mass exchangers, compressors, and ventilation devices),

- innovative cooling system design (e.g., energy efficient dehumidification systems, renewable energy assisted air-conditioning systems, and district cooling), 
- intelligent air-control strategies.

The design of both the cooling system and the air-control strategy is closely related to the indoor airflow fields, such as temperature fields, air velocity fields, and pressure fields.

For some special buildings, e.g., data centers, the indoor thermal maps, pressure fields, and airflow patterns have been commonly used to help the energy efficiency of thermal and airflow management [5-8]. For general buildings, one 'perfect' $\mathrm{H}-$ VAC system should be able to maintain the occupants' thermal comfort sensation while being energy efficient. Indoor thermal maps and airflow patterns have been widely used to estimate the distribution of indoor human thermal comfort index [9-15], and to quantify indoor architectural-design [15-17]. On the other hand, the indoor temperature observed from sensor networks was used as feedback information for indoor temperature control [18-21]. Indoor thermal maps and air velocity fields can be used to analyze indoor airflow for localized air-conditioning [22-25], which can maintain the thermal comfort and minimize the energy consumption of HVACs. However, it is not easy to obtain the real-time indoor physical fields, and a few publications [26-28] began to consider the airflow control directly using indoor physical fields (i.e., temperature field and air velocity field) as the feedback information. If we can obtain the real-time indoor physical fields and combine them with the indoor heat sources and occupancy information for the real-time localized air-conditioning, the energy consumption of HVACs may be further reduced. Unfortunately, in real applications, it is not easy to directly measure the indoor physical fields.

\section{Computational fluid dynamics}

The most popular tool to estimate indoor physical fields is the computational fluid dynamics (CFD) technique [29]. CFD technique can provide the dense 2dimensional and/or 3-dimensional data, such as temperature, pressure, and velocity by solving a series of partial differential equations (PDEs) using a numerical 
method [30]. An indoor temperature field (i.e., thermal map) and a 3-dimensional velocity streamline obtained from CFD simulations are shown in Figure 1.1 and Figure 1.2, respectively. CFD simulation can map the sampled spatial domain to the corresponding physical field data, once the model parameters and boundary condition are given. It can tell us the global information of a physical field which is rather expensive or even impossible to obtain from a real experiment.

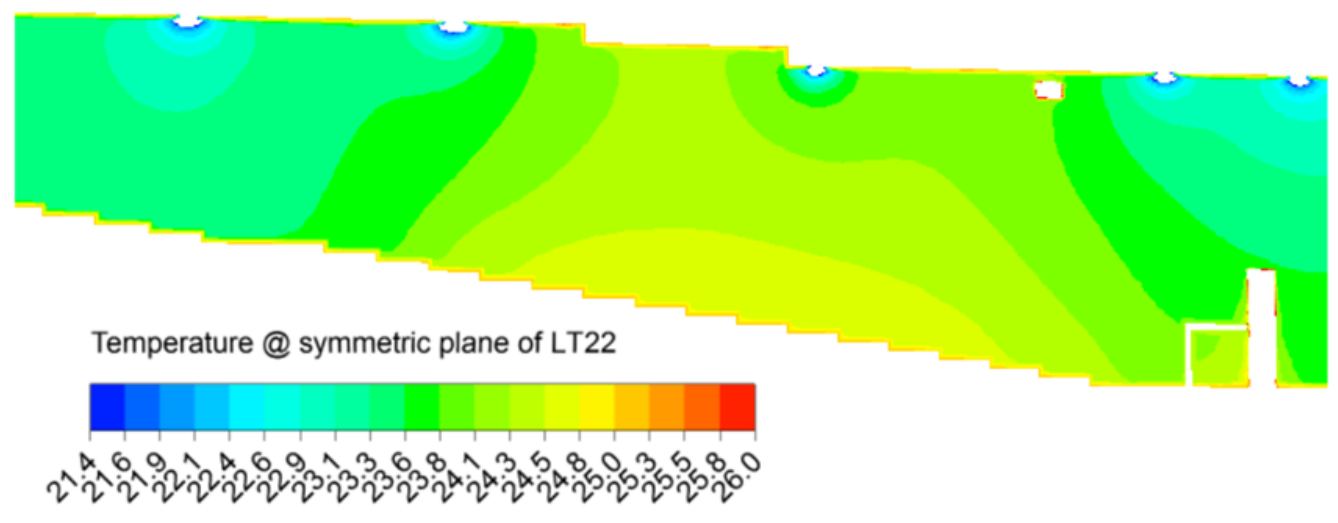

FiguRE 1.1: The temperature field (at degree centigrade) of the symmetric plane of Lecture Theatre 22 at Nanyang Technological University, Singapore.

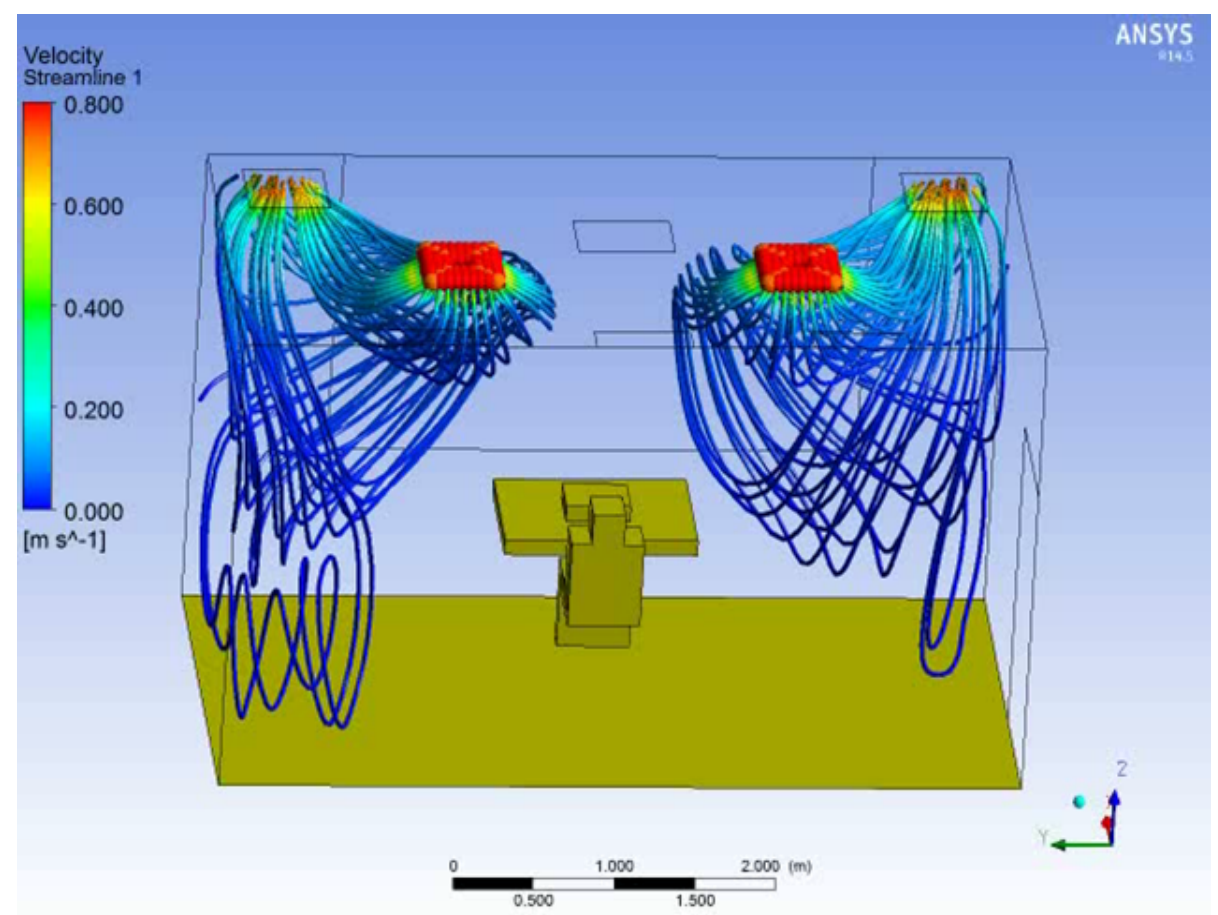

Figure 1.2: The velocity streamline of an office room obtained from CFD simulation. 
However, it is not easy to obtain reliable CFD simulation data due to the high computational cost and tedious calibration work. The accuracy of the CFD simulation strongly depends on the setting of boundary conditions and CFD models [31]. In practice, the real boundary conditions of the rooms or buildings are unavailable, and the idealized version is commonly used instead. For example, we may set certain temperature value for a wall for simplicity, but the real temperatures of deferent areas of the wall are probably different. Even though CFD modeling has been extensively researched, the reliability of CFD results remains a key concern when performing CFD simulations.

For many CFD applications, to produce reliable simulation results, we need to adjust the input parameters of the simulation to amend the agreement between the simulated results and the corresponding experimental data [32-34]. The input parameters can be boundary condition parameters [32], model parameters [32-34], etc. Tens to hundreds of simulation runs are required for the calibration process of finding acceptable model parameters. However, even a well calibrated CFD model cannot remove the gap between simulated and experimental data, which implies that the results of calibrated CFD models still have room for further improvement.

In addition, for the indoor environment, the input parameters are commonly varying due to the change of heat source distribution, the change of radiation condition, the change of the set-point of the air-conditioning system, etc. If the environment (e.g., heat sources) changes, which leads to the change of both the physical field and the input parameters of the CFD simulation, to estimate the indoor physical fields, we have to repeat the simulation. The time and computational cost of redoing the simulation for the varying parameters are unmanageable. Therefore, a new method which can quickly predict a physical field is highly desirable.

\section{Sensor observations}

Sensing indoor physical fields is quite difficult using general sensor networks because sensor networks can only observe the data at particular sparse locations. 
The thermal infrared cameras can provide a 2-dimensional thermal map of the surface of an object. It has been widely used for building diagnostics [35] and energy monitoring system [36]. The thermal infrared camera can record the radiant thermal energy emitted from an object and converse the heat flux to the temperature with the principle of heat transfer. But it cannot measure the air temperature and hence cannot provide the environmental temperature field.

Particle image velocimetry (PIV) measurement system can provide 2-dimensional or 3-dimensional indoor air velocity fields, which has widely used as the ground truth to verify the CFD simulation results $[37,38]$. However, when using a PIV system, the test-bed will be filled with seeding particles, which makes PIV systems suitable only for experimental environments and not appropriate for real scenarios.

Common sensors (e.g., temperature, pressure, and air velocity sensors) can only provide the single-spot measurements. Sensor networks can practically provide only sparse and isolated observations, but the observations are usually much more accurate than the CFD simulation results.

\subsection{Motivations}

Currently, the most popular way to estimate indoor physical fields, such as thermal maps and airflow patterns is the CFD simulations. However, the CFD simulation suffers from the following three issues:

- the simulation requires experts to carefully calibrate the CFD model;

- the simulation results are inaccurate compared with sensor observations;

- the simulation is computationally expensive and time-consuming.

Therefore, with only the CFD simulation, it is difficult to achieve an accurate estimation of the indoor physical fields of interest. In addition, as mentioned before, if the input parameters of the CFD simulation change, the physical field 
would change. To find the new physical field, the CFD simulation is required to be repeated. Since the indoor environment (e.g., the heat sources distribution) of most of buildings is usually irregular varying, we have to endlessly repeat the CFD simulations, which is quite tedious and time-consuming.

However, the indoor sensor networks can practically provide real-time accurate observations. Can we fuse the relatively inaccurate physical fields with the relatively accurate sensor observations? By fusing a simulated physical field and sparse sensor observations, one may find a better estimation of the real physical field. In addition, we are interested in the quick reconstruction of one physical field from sparse sensor observations. The main problem is how we can fuse the sparse sensor observations with simulated physical fields.

In the thesis, 'fusion' is accomplished in two fundamental ways:

1. the calibration of simulated physical fields using sparse sensor observations

2. the reconstruction of physical fields from sparse sensor observations and an off-line physical fields database obtained from CFD simulations.

The calibration work allows us to achieve a better estimation of indoor physical fields while the reconstruction work allows us to quickly obtain the estimation of indoor physical fields without doing CFD simulation. In the reconstruction work, one off-line database obtained from a group of CFD simulations is required. The calibration work can be used to enhance the simulated physical fields in the database.

The aim of this work is to directly estimate the indoor physical fields under various input parameters of CFD simulations from sparse sensor observations. In other words, if the input parameters (which correspond to the setting of air-condition systems, the power of heat sources, the heat sources distribution, etc.) change, the simulation is not required to be repeated, and we can quickly estimate the physical fields from sparse sensor observations. 
The ability to quickly reconstruct indoor physical fields can help us efficiently estimate the distribution of thermal comfort index and more precisely control the indoor airflow [11-14, 25]; therefore, improving the energy efficiency of HVACs. For data centers, the ability to quickly estimate the indoor thermal map and airflow patterns can make easy the energy efficient thermal management and task scheduling [6, 39-41].

The sensing locations play a key role in this physical field reconstruction problem. The spatial locations of the sensor nodes affect the error of the reconstructed physical field. The optimization of the sensing locations can even affect whether the reconstruction work can provide a reasonable solution or not. Finding the optimal sensor placement solution is a challenging combinatorial problem, which is typically NP-hard. It is usually too computationally expensive and even impossible to find the optimal solution. Hence, finding an effective suboptimal solution via computationally efficient method has achieved increasingly attention in recent years.

This dissertation provides a method to quickly estimate indoor physical fields from sparse sensor observations. To achieve this goal, we divide this work into the following three main steps, each of which corresponds to a research problem.

- Calibration of simulated physical fields with sparse sensor observations.

- Reconstruction of physical fields from sparse sensor observations.

- Sensor placement for the optimal physical field reconstruction.

Next, we review how the state-of-the-arts solve the three problems and indicate the room where the state-of-the-arts can be further improved. Following the literature review, we briefly present our methodologies and contributions to solve the three problems. 


\subsection{Literature review}

\subsubsection{Calibration of the simulated physical field with sparse sensor observations}

CFD simulation is the most popular tool to estimate indoor physical fields. But the simulation results are inaccurate compared with sensor observations. Currently, the CFD calibration refers to the process of adjusting turbulence model and input parameters of CFD simulations to minimize the gap between the simulation results and the experimental data [32-34, 42-45]. The error of the physical field estimated from a CFD simulation comes from:

- the assumptions in the physical model,

- the numerical approximation of the computational model,

- the choice of turbulent model,

- the ideal assumptions and uncertainty of the input parameters.

In practice, we do not know the exact mathematical model of the indoor dynamic airflow. The mathematical model used in a CFD simulation is an idealized physical model under some assumptions, for example, the assumption on the viscosity of airflow, and the assumptions on boundary conditions [30].

The numerical error is one of the main reasons for the inaccurate simulation results. The numerical model calibration refers to select proper numerical method (e.g., finite element method and finite volume method) and adjust grid generation to guarantee the numerical model can well match with the mathematical model. The grid generation is to discrete the spatial space of the indoor environment. The resolution of the mesh, i.e., the grid, is closely related both the quality of the numerical model and the computational cost. The adjustment of the grid generation is to balance the quality of numerical model and the computational cost of the 
CFD simulation [32, 42]. A full description of the numerical model calibration can be found in [44].

The choice of turbulent model also has a great influence on the simulated indoor physical field, Examples on the comparison of different turbulent models can be found in $[43,45]$.

Many current works have focused on the calibration of the input parameters of the simulation [32-34, 42]. The input parameters include the boundary condition parameters (e.g., the wall temperature, the inlet cooling air velocity, the heat sources, etc.) and some model parameters (e.g., the turbulent parameters). In practice, the real boundary conditions of the rooms or buildings are unavailable and the idealized version is used instead. For example, the temperature of a wall in many rooms is nonuniform, but for simplicity, in many applications, the wall temperature was assumed to be uniform and was set as a constant value, i.e., the observed temperature of certain point of the wall $[11,15,46]$.

The current CFD calibration work [32-34, 42-45] and the references therein mainly focused on the calibration of CFD model and its input parameters to narrow the gap between simulation results and sensor observations. To produce reliable simulation results, we need to properly set the computational model (mathematical and/or numerical models) and adjust the input parameters to amend the agreement between the simulated results and the corresponding experimental data [32-34]. Tens to hundreds of simulation runs are required for the calibration process of finding acceptable input parameters. All the calibration work are very computationally expensive and time-consuming. Even a well-calibrated CFD model with the optimal input parameters cannot remove the gap between simulated and experimental data, which implies that the results of calibrated CFD models still have room for further improvement.

To the best of our knowledge, no reported publication has focused on how to correct the simulated physical field with the experimental data, i.e., the sparse sensor observations. In this work, we directly calibrate the results of CFD simulations. 
The proposed methodology can be applied for general CFD simulations and be a valuable additional step of the standard CFD simulation procedure, which can further narrow the gap between the simulation results and the experimental data.

\subsubsection{Physical field reconstruction from sparse sensor ob- servations}

CFD simulation can provide an estimation of the indoor physical fields of interest. Both the simulation and the calibration work are computationally expensive and time-consuming, which motivate us to directly estimate the physical fields from sparse sensor observations. Sensor nodes usually can provide real-time observations. It is possible to merge the numerical model of a physical field with sparse sensor observations to estimate the physical field. However, it is still computationally expensive and current work mainly focused on simple 1-dimensional/2-dimensional toy examples [47-49].

Indoor physical fields are with 'dense' data. The reconstruction of a physical field from sparse observations is an ill-posed problem. Model-order-reduction techniques can be used to solve this ill-posed problem.

Principal component analysis (PCA) is also known as proper orthogonal decomposition in the fluid mechanics community. It is a powerful tool to represent high dimensional data by a low dimensional description [50]. A finite dimensional signal can be approximated by a combination of the dominant PCA modes whose number is much less than the dimension of the signal; therefore, once the dominant PCA modes are known, this signal can be adequately determined by the dominant PCA coefficients. But directly computing the PCA modes is difficult. To efficiently find them, Sirovich developed the method of snapshots, which was later extended and widely applied to various applications [51].

Two approaches are commonly used to estimate the PCA coefficients of one physical field of interest. 
- One approach is to find regression models to estimate the PCA coefficients from the observed input parameters of interest [53-56]. These parameters can be Rayleigh number [53], Mach number [54], parametric boundary conditions $[55,56]$, etc. With the observed input parameters, the PCA coefficients can be estimated from the regression model. Most of the current works [53-55] used the spline interpolation technique to train the regression models, which are multi-input-single-output (MISO) functions. For each PCA coefficient, we need to train a regression model. The spline interpolation technique has been widely used for regression problems. It can provide very good fitting performance if the number of input variable is less than four, while if the input variable are more than three, this technique may not be a good choice $[52]$.

- The other approach is to solve a linear equation formulated by sparse observations of the physical field [57-59]. With the PCA modes, we can easily find a linear measurement model for every single point of the physical field in terms of the PCA coefficients. If enough sensor observations are available, we can easily estimate the dominant PCA coefficients. To solve this linear inverse problem, the number of sensor observations cannot be less than the number of dominant PCA modes, and the sensing locations are very important in terms of the estimation performance.

The two approaches find PCA coefficients from the observed input parameters and sparse sensor observations, respectively. In the case that both the input parameters and sensor observations are available, how to fuse them to achieve a better estimation of the indoor physical field? If the number of sensor observations is less than the number of dominant PCA modes, how to estimate the indoor physical field? To the best of our knowledge, no reported publications have answered the two questions. 


\subsubsection{Sensor placement for the optimal physical field re- construction}

Sensor placement plays a critical role in the physical field reconstruction problem. Sensor placement is to seek the least number of required sensor nodes and their corresponding sensing locations within a known spatial domain such that the estimation accuracy can meet the requirement. Specifically, assuming that the observation models of all potential sensing locations are known, we want to determine the least number of required sensors with which the physical field of interest can be recovered within a predefined accuracy.

Obviously, one straightforward method is to evaluate the performance of all possible combinations of all potential sizes of the candidate sensing locations, and then select the one with the least number of sensor nodes that satisfies the required estimation accuracy. But such a combinatorial approach is computationally intractable. In practice, the direct enumeration is impossible since the number of potential sensing locations is large. Apart from the enumeration method, the optimal solution can also be obtained by branch-and-bound methods [62, 63], which unfortunately do take a very long time, even for a moderate scale problem [64]. Consequently, in recent years the sensor placement for physical field reconstruction has attracted increasing attention to find a suboptimal solution via computationally more efficient methods [59, 64-75].

Heuristics have been proposed to reduce the cost of the exhaustive search. The simplest one is to place sensor nodes at the spatial maxima and minima of proper orthogonal components of the physical field of interest [72]. This method is simple but only suitable for some special cases [59]. Other heuristics include genetic algorithms [73], particle swarm optimizer [74], tabu search [74], and cross-entropy optimization [75]. They all involve a prohibitive computational cost, and the solutions have no optimality guarantee. 
Joshi and Boyd [64] formulated the sensor placement problem as an elegant nonconvex optimization problem, and approximated it as a convex optimization problem by the relaxation of the nonconvex Boolean constraints that represent the sensor placements, to a convex box set. This convex relaxation was then used in many works $[69,70,76-79]$. The sensing locations can be easily determined based on the solution of the convex optimization problem. But the sensor placement may lead to an ill-conditioned observation model due to the gap between the nonconvex and the convex optimization problems, especially when the number of sensor nodes is very limited. Such a result has been shown to be no better than other works $[65,78,79]$. However, the authors in [64] provided a local optimization technique to improve the result. This technique is computationally expensive but some numerical examples showed that with the local optimization, the convex relaxation method can indeed provide very good results.

The sensor placement problem was also solved by some greedy algorithms in which the sensor locations are individually determined by optimizing some proxies of the error of the estimated physical field, such as the determinant of Fisher information matrix [71], and the condition number [59,67,68] or the frame potential [65] of the observation matrix. The $\eta$-confidence ellipsoid of the estimation error depends on the determinant of the Fisher information matrix [64], which was optimized using one greedy method in [71], but it is shown to be no better than other methods in the examples in [65]. For the sensor placement problem, the minimum requirement of the solution is that the observation model should be well-conditioned. Therefore, some researchers determined the sensing locations by minimizing the condition number of the observation matrix [59, 67, 68]. However, the condition number is a concept for a nonsingular matrix, and we need to firstly determine a group of sensing locations to guarantee that the observation matrix is nonsingular [67], which is, unfortunately, a combinatorial problem. Additionally, the minimum condition number of the observation matrix does not mean the minimum estimation error except when all the observation vectors have the same norm because the sensing energy should be considered, which is related to the signal-to-noise ratio. 
Recently, Ranieri et al. [65] provided a novel greedy algorithm by minimizing the frame potential of the observation matrix. This method is computationally efficient but: 1) like the condition number minimization, it is only effective for the case where all the observation vectors have the same norm; 2) it cannot guarantee that the observation matrix is well-conditioned.

All the above-mentioned works focused on the case where the number of sensor nodes is fixed. One sparse-promoting technique has been used to minimize the number of required sensor nodes by adding a sparsity-promoting penalty term to the cost function [69]. This method works well when the dimension of the estimated parameter (i.e., the PCA coefficients) is small (e.g., the dimension is set as 2 in the example of Ref. [69]). However, if the dimension of the estimated parameter is large (e.g., a few tens, which is very common in physical field reconstruction problems $[59,67,80])$, this method will be ineffective in determining the least number of required sensor nodes. Therefore, a new computationally efficient algorithm that can both determine the minimum number of required sensor nodes and provide a better sensor configuration is very attractive.

\subsection{Methodology}

\section{Calibration of the simulated physical field with sparse sensor observations}

Since sensor observations are much more accurate than simulation results, we ignore the difference between sensor observations and the real values. We use the sparse sensor observations as the ground truth. In addition, we assume that the simulation results can provide the rough global profile of the real physical fields, i.e., the profile of the simulated physical field is similar to that of the real physical field. If the CFD model is well-calibrated, this assumption can be easily satisfied. 


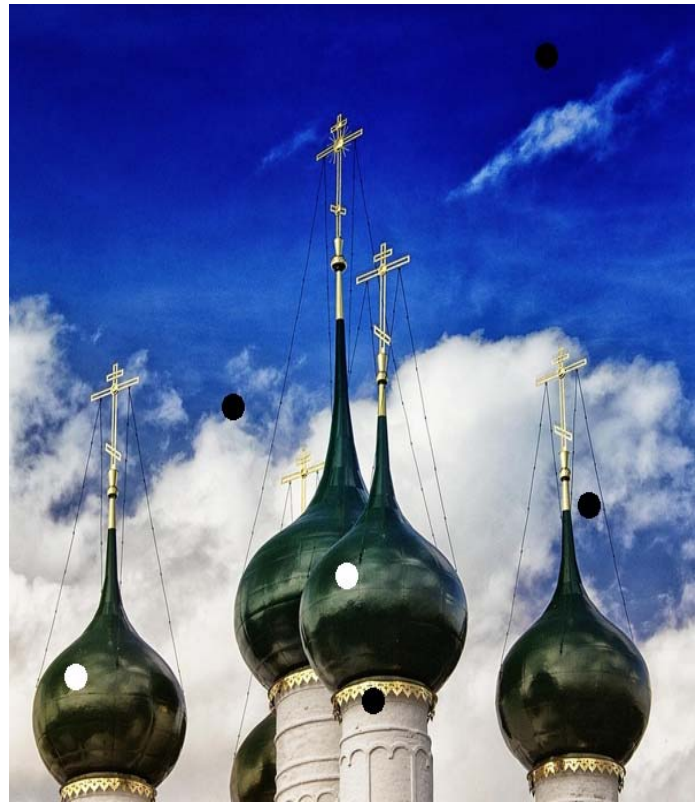

(A) Input image and control samples

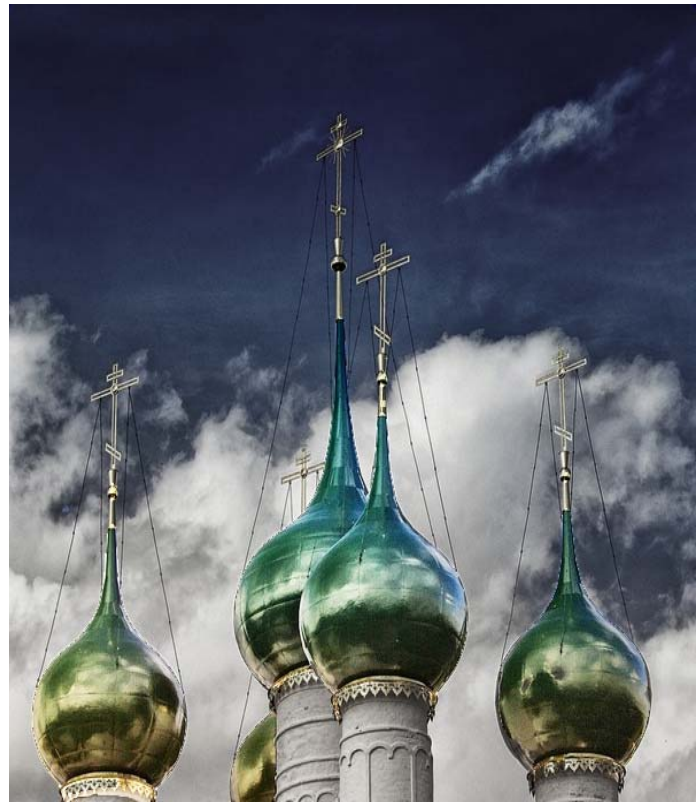

(B) Editing result

Figure 1.3: An simple example of image editing. The dots in the left image are used as control samples to edit the input image.

We have taken inspiration from the image editing technique [81, 82]. As shown in Figure 1.3, an image can be globally edited with sparse control samples. The right image is fused by the input image and the sparse control samples. This example is found from [82]. The result of image editing reserves the content of the original image but with the style of the sparse control samples. For the calibration of the indoor physical fields obtained from CFD simulations, we can view the sparse sensor observations as the control samples. The calibration problem can be viewed as a physical field editing problem.

Like the image editing problem, we formulate the calibration of the simulated physical field as an optimization problem. The objective function is a sum of two parts. One part is responsible for narrowing the difference between sensor observations and simulation results, while the other part guarantees the similarity between the CFD simulation results and the calibration results. The optimization problem is solved by a numerical method. To test the proposed method, we did a CFD simulation for an air-conditioned room in which we arranged eight temperature sensors. With the help of four sensor observations, an indoor thermal map obtained from 
the CFD simulation was well calibrated. The other four sensor observations were used to test the performance of the calibration.

\section{Physical field reconstruction from sparse sensor observations}

In this work, we consider the case that both the input parameters of interest and some sparse locations of the physical field are observed. We present a twostage physical field reconstruction (TSPFR) method by combining the two current approaches to achieve better estimation result. The new approach can quickly reconstruct the physical field of interest. The block diagram of the new approach is shown in Figure 1.4.

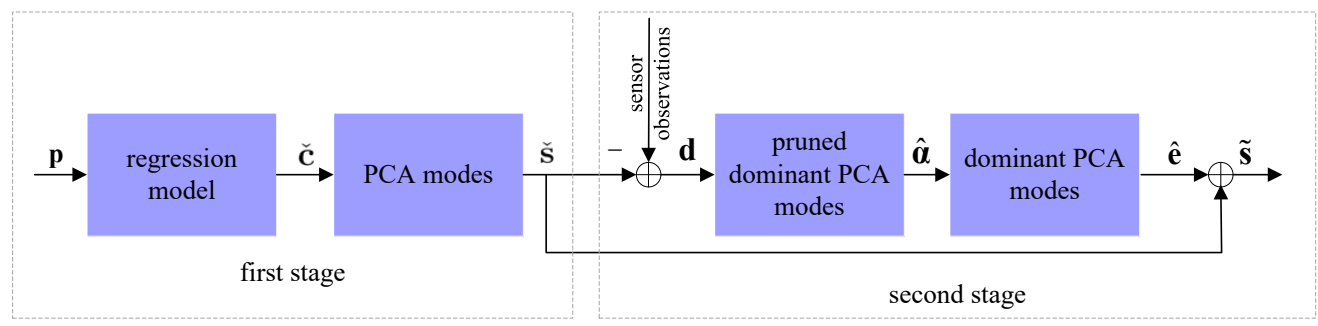

FiguRE 1.4: The block diagram of the two-stage physical field reconstruction

Using the method of snapshots [60], we can easily find the PCA modes from the known physical field database obtained from off-line CFD simulations under various input parameters.

In the first stage, we train a regression model to estimate the PCA coefficients from the observed input parameters of CFD simulations. In previous work, spline interpolation [53-55] was used to train the regression models. To find a better regression model, we propose a scaled extreme learning machine (sELM) algorith$\mathrm{m}$, which is a variation of the standard ELM algorithm [61]. Here, sELM is a powerful approach to train a neural network, which is a multi-input-multi-output (MIMO) regression model. Compared with the spline interpolation, we train only one regression model when using sELM. With the observed input parameters, we 
can estimate all the PCA coefficients with which we can approximately reconstruct the indoor physical field.

Compared with the physical field reconstructed in the first stage, sensor observations of the field are considered to be more accurate. However, the observations only provide information of the sensing locations. For simplicity, we introduce an error field, i.e., the difference between the indoor physical field reconstructed in the first stage and the real field. With the sensor observations, we can obtain a linear measurement model of the error field.

In the second stage, we can obtain the dominant PCA coefficients of the error field from the measurement model by solving a least square problem. Then, we can reconstruct the error field with which we can modify the physical field estimated in the first stage. Such a modification is to correct the dominant PCA components of the physical field reconstructed in the first stage.

In addition, in the first stage, some of the PCA components may be well estimated, which are not required to be corrected in the second stage. The leave-one-outcross-validation (LOOCV) technique can be used to test the regression model and determine the PCA components which can be well-estimated in the first stage. We can simply remove the PCA components that are well-estimated in the first stage from the low-dimensional approximation of the error field in the second stage. As mentioned before, the number of sensor observations cannot be less than the number of PCA modes used in the second stage. In this case, the number of PCA modes used in the second stage is reduced. Hence, we can reduce the number of sensor observations.

We apply the proposed TSPFR approach to estimate the indoor thermal maps of one air-conditioned room. The results demonstrate that compared with the current one-stage methods, the proposed two-stage method can provide better estimation of the thermal map with less sensor observations. 


\section{Sensor placement for the optimal physical field reconstruc- tion}

Sensor placement can be formulated as a combinatorial problem. The exhaustive searching approaches are computationally intractable and impractical. In this work, We propose a new greedy algorithm to minimize the number of required sensor nodes and determine their locations for the physical field reconstruction problem such that the estimation error meets the requirement. The new method is called maximal projection on minimum eigenspace(MPME).

The measurement models of all potential sensing locations are a prior known, which can be obtained from the dominant PCA modes. Each measurement model is a linear equation in terms of the PCA coefficients. We select the sensing locations oneby-one. In this way, the least number of required sensor nodes can be determined by checking whether the estimation accuracy is satisfied after each sensing location is determined. The minimum eigenspace is defined as the eigenspace associated with the minimum eigenvalue of the dual observation matrix. For each sensing location, the projection of its observation vector onto the minimum eigenspace is shown to be monotonically decreasing with respect to the worst case error variance (WCEV) of the estimated parameters, i.e., the PCA coefficients.

We select the sensing location whose observation vector has the maximum projection onto the minimum eigenspace of the current dual observation matrix. The proposed MPME is shown to be one of the most computationally efficient algorithms. Our Monte-Carlo simulations showed that MPME outperforms the convex relaxation method [64], the SparSenSe method [69], and the FrameSense method [65] in terms of WCEV and the mean square error (MSE) of the estimated parameters, especially when the number of available sensor nodes is very limited. 


\subsection{Outline and contributions}

The aim of this work is to quickly estimate an accurate indoor physical field of interest from sparse sensor observations. To achieve the goal, we develop the following three research problems.

- Calibration of simulated physical fields with sparse sensor observations.

- Reconstruction of physical fields from sparse sensor observations.

- Sensor placement for the optimal physical field reconstruction.

For each of the three research problems, we use a chapter to detail it in this dissertation. In the following, we present a brief summary of each chapter and its contributions.

In Chapter 2, we develop the problem of calibrating simulated physical fields with sparse sensor observations. For this work:

1. We propose a mathematical formulation of the simulated physical field calibration problem.

2. We formulate the proposed calibration problem as an optimization problem inspired by the image editing problem.

3. We provide a numerical method to solve the optimization problem. With the proposed method, we can obtain an explicit solution of the calibrated physical field in which we need to solve the inverse of a matrix with a very large dimension.

4. We provide a computationally efficient method to find the approximated solution of the inverse of the matrix.

5. We apply the proposed methodology to an air-conditioned room. We use four sensor observations to calibrate a thermal map with $167 \times 365$ data points. The experimental results show the effectiveness of the proposed method. 
In Chapter 3, we focus on the reconstruction of indoor physical fields. We fuse the information of observed input parameters of the CFD simulation and the sparse observations of the physical field to achieve better estimation. For this work:

1. We propose a two-stage physical field reconstruction (TSPFR) approach to estimate an indoor physical field from the observed input parameters of the CFD simulation and sparse sensor observations of the physical field.

2. In the first stage, we build a regression model to estimate all PCA coefficients of interest. To find this regression model, we provide an sELM algorithm which is shown better and more convenient than the commonly used spline interpolation method.

3. In the second stage, we use sparse sensor observations to modify the dominant PCA coefficients and therefore improve the estimation accuracy.

4. We theoretically show why the proposed TSPFR approach outperforms the two current one stage approaches.

5. Using the proposed TSPFR method for physical field reconstruction, the number of required sensor observations can be less than the number of dominant PCA modes.

6. We apply the proposed TSPFR approach to estimate the indoor thermal maps of one air-conditioned room. The results demonstrate that compared with the current one-stage methods, the proposed two-stage method can provide a better estimation of the thermal map with fewer sensor observations.

In Chapter 4, we try to find the optimal sensing locations for the physical field reconstruction problem. Many publications have focused on this problem. We propose a new algorithm, named maximal projection on minimum eigenspace (MPME), which has the following advantages:

1. The MPME is computationally one of the most efficient sensor placement algorithms. 
2. The MPME can readily determine the minimum number of required sensor nodes.

3. The storage requirement of the MPME is much less than the state-of-the-arts. In the example of indoor thermal map estimation, the convex relaxation, the SparSenSe and the FrameSense methods cannot work on a workstation with 16GB RAM due to the shortage of memory. However, the proposed MPME algorithm can immediately provide the solution in a laptop with 2GB RAM.

4. The MPME outperforms the convex relaxation method [64], the SparSenSe method [69], and the FrameSense method [65] in terms of the WCEV and the mean squared error (MSE) of the estimated parameters, especially when the number of available sensor nodes is very limited.

5. The MPME can guarantee that the observation matrix is well-conditioned but the convex relaxation, the SparSenSe and the FrameSense methods cannot guarantee such a condition, especially when the number of available sensor nodes is very limited.

6. If the observation vectors of all potential sensing locations have the same norm, the FrameSense with local optimization [64] can provide the best solution. But this assumption is not true for general physical field reconstruction problem. The observation vector is one row of the matrix consists of all dominant PCA modes. The rows of this matrix do have different norms. For general sensor placement problems, the MPME without local optimization outperforms the state-of-the-art with local optimization.

In Chapter 5, we summarize the thesis and discuss various directions for future work. 



\section{Chapter 2}

\section{Calibration of simulated physical fields}

To estimate one physical field, the computational fluid dynamics (CFD) simulation is the most popular tool, especially for the indoor environment. Because of the mismatch between the real physical model and the idealized mathematical model, the computational error, and the error of the observed input parameters, the simulated physical fields always have a gap with the experimental results. To narrow the gap, researchers in this community mainly focused on the improvement of the numerical methods and the calibration of the input parameters. In this chapter, we develop a method to calibrate the simulated physical fields with sparse sensor observations, which can narrow the gap between the simulation results and the experimental results. To the best of our knowledge, no reported publication has focused on this problem.

This chapter is organized as follows. In Section 2.1, we formulate the simulated physical field calibration problem. In Section 2.2, we detailed the proposed methodology and formulate the calibration problem as an optimization problem. In Section 2.3, we present a numerical method to solve the optimization problem. We then provide a computational efficient approximated solution in Section 2.4. In 
Section 2.5, we calibrated a thermal map of an air-conditioned room to verify the effectiveness of the proposed method. The conclusions are given in Section 2.6.

\subsection{Problem formulation}

We denote a real physical field (e.g., thermal map, airflow pattern, and pressure field) of interest by $f(\mathbf{x})$, where $\mathbf{x}=(x, y, z) \in \Omega$ with $\Omega$ being the domain of interest. The physical field obtained from CFD simulation is a numerical solution, which can be simply interpolated to be a continuous one. We denote a continuous physical field interpolated from a CFD solution by $f_{c}(\mathbf{x})$, which can be described as

$$
f_{c}(\mathbf{x})=f(\mathbf{x})+v(\mathbf{x}), \quad \mathbf{x} \in \Omega
$$

where $v(\mathbf{x})$ represents the error of the simulated physical field. We assume that $\max _{\mathbf{x} \in \Omega} v(\mathbf{x}) \leq v_{\max }$, and $v_{\max }$ is a small value compared with the magnitude of $f(\mathbf{x})$.

We denote one of the sensor observations of the physical field by

$$
s_{k}=f\left(\mathbf{x}_{k}\right)+n_{k}, \quad \mathbf{x}_{k}=\left(x_{k}, y_{k}, z_{k}\right) \in \Omega_{s} \subset \Omega, k=1,2, \ldots m
$$

where $n_{k}$ is the measurement noise of the $k$-th observation, $\Omega_{s}$ is the set of all sensing locations, and $m$ is the number of available sensor observations. Without loss of generality, we assume that $\left|n_{k}\right| \ll\left|v\left(\mathbf{x}_{k}\right)\right|$ for all $1 \leq k \leq m$.

In practice, $f_{c}(\mathbf{x}), \mathbf{x} \in \Omega$ and $s_{k}, 1 \leq k \leq m$ are known. In what follows, we estimate $v(\mathbf{x}), \mathbf{x} \in \Omega$ based on $f_{c}(\mathbf{x}), \mathbf{x} \in \Omega$ and $s_{k}, 1 \leq k \leq m$. With the estimated $v(\mathbf{x})$, we can easily correct the simulated physical field $f_{c}(\mathbf{x})$. 


\subsection{The proposed methodology}

In this section, we formulate the problem of calibrating a simulated physical field to be an optimization problem.

Since $\left|n_{k}\right| \ll\left|v\left(\mathbf{x}_{k}\right)\right|$ for all $1 \leq k \leq m$, we ignore the measurement noise. We denote the error of the simulated physical field at the sensing locations by $e_{k}$, and from (2.1) and (2.2) we can obtain

$$
e_{k}=f_{c}\left(\mathbf{x}_{k}\right)-s_{k}=v\left(\mathbf{x}_{k}\right)-n_{k} \approx v\left(\mathbf{x}_{k}\right), 1 \leq k \leq m
$$

In the remainder of this chapter, we ignore the measurement noise and view $e_{k}$ as the real error of $f_{c}\left(\mathbf{x}_{k}\right)$.

To estimate the error of the simulated physical field $f_{c}(\mathbf{x})$, i.e., $v(\mathbf{x})$, we minimize the following cost function

$$
J_{0}=\int_{\Omega} \sum_{k=1}^{m} w\left(\mathbf{x}, \mathbf{x}_{k}\right)\left[v(\mathbf{x})-e_{k}\right]^{2} \mathrm{~d} \mathbf{x}+\lambda \iint_{\Omega \times \Omega} w(\mathbf{x}, \mathbf{y})[v(\mathbf{x})-v(\mathbf{y})]^{2} \mathrm{~d} \mathbf{x} \mathrm{d} \mathbf{y}
$$

where

$$
w(\mathbf{x}, \mathbf{y})=\exp \left(-\left\|f_{c}(\mathbf{x})-f_{c}(\mathbf{y})\right\|^{2} / \sigma_{m}\right) \exp \left(-\|\mathbf{x}-\mathbf{y}\|^{2} / \sigma_{d}\right)
$$

is a priori known weight, which can be directly calculated from the simulated physical field. Note that $w(\mathbf{x}, \mathbf{y})$ represents the affinity between $v(\mathbf{x})$ and $v(\mathbf{y})$. Both $\sigma_{m}$ and $\sigma_{d}$ are positive, which will be elaborated later. It is obvious that $w(\mathbf{x}, \mathbf{y}) \in[0,1]$ and that $w(\mathbf{x}, \mathbf{y})$ is very small unless the locations $\mathbf{x}$ and $\mathbf{y}$ are near and their corresponding physical value $f_{c}(\mathbf{x})$ and $f_{c}(\mathbf{y})$ are similar.

Solving the minimization problem (2.3), we can find an estimation $v(\mathbf{x})$, which we denote by $\hat{v}(\mathbf{x})$. With $\hat{v}(\mathbf{x})$, we can calibrate the simulated physical field $f_{c}(\mathbf{x})$, and the calibration result is

$$
\hat{f}(\mathbf{x})=f_{c}(\mathbf{x})-\hat{v}(\mathbf{x})
$$


The cost function (2.3) is the summation of two parts. The first part is responsible for the regions around sensing locations. In the region around $\mathbf{x}_{k}$, the value of $v(\mathbf{x})$ is forced to be similar to $e_{k}$, especially when $f_{c}(\mathbf{x})$ is similar to $f_{c}\left(\mathbf{x}_{k}\right)$. Consequently, in the region around $\mathbf{x}_{k}$, the physical value is calibrated to be similar to the sensor observation $s_{k}$, especially for the subregion in which $f_{c}(\mathbf{x})$ is similar to $f_{c}\left(\mathbf{x}_{k}\right)$. In this way, in the region around $\mathbf{x}_{k}, \hat{f}(\mathbf{x})$ preserves the profile of $f_{c}(\mathbf{x})$ but the exact values are adjusted to be similar to the sensor observation $s_{k}$. The first term in (2.3) can guarantee a good local calibration for the simulated physical field, but it has insignificant influence on the regions far away from $\mathbf{x}_{k}$ for all $1 \leq k \leq m$, in which $w\left(\mathbf{x}, \mathbf{x}_{k}\right) \approx 0$.

On the other hand, the second term has a global influence, which can be explained from the following two perspectives: 1) the second term can transmit the adjustment from the local regions around the sensing locations to the whole physical field; 2 ) the second term can guarantee that in any local region, the gradient of $\hat{v}(\mathbf{x})$ is small if in that region the simulated values $f_{c}(\mathbf{x})$ are similar, which guarantees the profile of $\hat{f}(\mathbf{x})$ is similar to that of the simulated physical field $f_{c}(\mathbf{x})$.

The optimization problem is controlled by three parameters: $\lambda, \sigma_{m}$, and $\sigma_{d} . \lambda$, which we call the balance factor, can balance the contribution of the two terms in (2.3). A small $\lambda$ leads to good calibration in the local regions around sensing locations, while a large $\lambda$ can globally reduce the gradient of the adjustment at the cost of the calibration accuracy in the local regions around the sensing locations.

In Equations (2.3)-(2.4), w(x, y) is the product of the following two Gaussian functions, with $\sigma_{m}$ and $\sigma_{d}$ controlling the rate of decay of the two gaussian functions, respectively.

$$
\begin{aligned}
& \exp \left(-\left\|f_{c}(\mathbf{x})-f_{c}(\mathbf{y})\right\|^{2} / \sigma_{m}\right) \\
& \exp \left(-\|\mathbf{x}-\mathbf{y}\|^{2} / \sigma_{d}\right)
\end{aligned}
$$

We call $\sigma_{m}$ the magnitude variance, which mainly depends on the dynamic range of the simulated physical field. If the local gradient of the physical field is large, a 
large $\sigma_{m}$ is proper. Otherwise the first Gaussian function will be very small which may dilute the influence of the second Gaussian function. On the other hand, if the thermal map has a low dynamic range, a relatively small $\sigma_{m}$ is proper.

We call $\sigma_{d}$ the distance variance. It controls the scope of the local region around one of the sensing locations in which the physical data can be calibrated by minimizing the first term in (2.3). In other words, in the particular local region, the sensor observation have a significant influence on the physical field calibration.

\subsection{Numerical method to solve the optimization problem}

It is difficult for us to directly minimize $J_{0}$ in (2.3) to find the estimation of the error, i.e., $v(\mathbf{x}), \mathbf{x} \in \Omega$. Numerical methods can make the optimization problem easily solvable.

We denote the discrete space of the domain $\Omega$ in the CFD simulation by $\Omega_{D}=$ $\left\{\mathbf{y}_{1}, \mathbf{y}_{2}, \ldots, \mathbf{y}_{N}\right\}$. We assume that $\Omega_{s} \subset \Omega_{D}$, which implies that each sensing location is constrained to be one of the mesh points. Hence, we can denote $\mathbf{x}_{k}=\mathbf{y}_{l_{k}}$ and $\Omega_{s}=\left\{\mathbf{y}_{l_{1}}, \mathbf{y}_{l_{2}}, \ldots \mathbf{y}_{l_{m}}\right\}$, where $l_{k}$ is the mesh index of the $k$-th sensing location. Then, we can approximate the cost function $J_{0}$ in (2.3) as

$$
J_{0} \approx J_{1}=\sum_{i=1}^{N} \sum_{k=1}^{m} w_{i l_{k}}\left(v_{i}-e_{k}\right)^{2}+\lambda \sum_{i}^{N} \sum_{j}^{N} w_{i j}\left(v_{i}-v_{j}\right)^{2}
$$

where $w_{i j}=w\left(\mathbf{y}_{i}, \mathbf{y}_{j}\right)$ and $v_{i}=v\left(\mathbf{y}_{i}\right)$. We introduce a new matrix $\mathbf{W}=\left[w_{i j}\right]$, i.e., $w_{i j}$ is the entry of $\mathbf{W}$ located at the $i$-th row and $j$-th column. 
For a more elegant description,we use the following cost function $J$ instead of $J_{1}$

$$
\begin{aligned}
J= & \frac{J_{1}}{\lambda}=\frac{1}{\lambda} \sum_{i=1}^{N} \sum_{k=1}^{m} w_{i l_{k}}\left(v_{i}-e_{k}\right)^{2}+\sum_{i}^{N} \sum_{j}^{N} w_{i j}\left(v_{i}-v_{j}\right)^{2} \\
= & \frac{1}{\lambda} \sum_{k=1}^{m}\left(\mathbf{v}-e_{k} \mathbf{1}\right)^{\mathrm{T}} W_{l_{k}}^{\mathrm{D}}\left(\mathbf{v}-e_{k} \mathbf{1}\right)+\sum_{j=1}^{N} \mathbf{v}^{\mathrm{T}}\left(I-A_{j}\right)^{\mathrm{T}} W_{j}^{\mathrm{D}}\left(I-A_{j}\right) \mathbf{v} \\
= & \mathbf{v}^{\mathrm{T}}\left(\frac{1}{\lambda} \sum_{k=1}^{m} W_{l_{k}}^{\mathrm{D}}+\sum_{j=1}^{N}\left(I-A_{j}\right)^{\mathrm{T}} W_{j}^{\mathrm{D}}\left(I-A_{j}\right)\right) \mathbf{v}-\left(\frac{2}{\lambda} \sum_{k=1}^{m} e_{k} \mathbf{w}_{l_{k}}^{\mathrm{T}}\right) \mathbf{v} \\
& +\frac{1}{\lambda} \sum_{k=1}^{m} e_{k}^{2} \operatorname{Tr}\left(W_{l_{k}}^{\mathrm{D}}\right) \\
= & \mathbf{v}^{\mathrm{T}} H \mathbf{v}-2 \mathbf{b}^{\mathrm{T}} \mathbf{v}+\mathbf{c}
\end{aligned}
$$

where $\mathbf{v}=\left[v_{1}, v_{2}, \ldots, v_{N}\right]^{\mathrm{T}}, \mathbf{1} \in \mathbb{R}^{N}$ is a vector with all 1 entries, $W_{j}^{\mathrm{D}}=\operatorname{diag}\left\{\mathbf{w}_{j}\right\}$, $\mathbf{w}_{j}$ is the $j$-th column of $\mathbf{W}$, and $A_{j}$ is a matrix with all 1 entries in $j$-th column and all 0 entries in others. Here, $\operatorname{diag}\{\cdot\}$ and $\operatorname{Tr}(\cdot)$ are diagonal matrix and trace operators, respectively. It is clear that

$$
\mathbf{b}=\frac{1}{\lambda} \sum_{k=1}^{m} e_{k} \mathbf{w}_{l_{k}}
$$

and

$$
\begin{aligned}
H & =\frac{1}{\lambda} \sum_{k=1}^{m} W_{l_{k}}^{\mathrm{D}}+\sum_{j=1}^{N}\left(I-A_{j}\right)^{\mathrm{T}} W_{j}^{\mathrm{D}}\left(I-A_{j}\right) \\
& =\frac{1}{\lambda} \sum_{k=1}^{m} W_{l_{k}}^{\mathrm{D}}+\sum_{j=1}^{N} W_{j}^{\mathrm{D}}-A_{j}^{\mathrm{T}} W_{j}^{\mathrm{D}}-W_{j}^{\mathrm{D}} A_{j}+A_{j}^{\mathrm{T}} W_{j}^{\mathrm{D}} A_{j} \\
& =\frac{1}{\lambda} \sum_{k=1}^{m} W_{l_{k}}^{\mathrm{D}}+\operatorname{diag}\left\{2 \sum_{j=1}^{N} \mathbf{w}_{j}\right\}-2 \mathbf{W} \\
& =D-W_{s}
\end{aligned}
$$


where

$$
\begin{aligned}
\sum_{j=1}^{N} W_{j}^{\mathrm{D}} & =\operatorname{diag}\left\{\sum_{j=1}^{N} \mathbf{w}_{j}\right\} \\
\sum_{j=1}^{N} A_{j}^{\mathrm{T}} W_{j}^{\mathrm{D}} & =\mathbf{W}^{\mathrm{T}}=\mathbf{W} \\
\sum_{j=1}^{N} W_{j}^{\mathrm{D}} A_{j}^{\mathrm{T}} & =\mathbf{W} \\
A_{j}^{\mathrm{T}} W_{j}^{\mathrm{D}} A_{j} & =\operatorname{diag}\left\{\sum_{j=1}^{N}\left(\mathbf{w}_{j}^{\mathrm{r}}\right)^{\mathrm{T}}\right\}=\operatorname{diag}\left\{\sum_{j=1}^{N} \mathbf{w}_{j}\right\}
\end{aligned}
$$

Here, $\mathbf{w}_{j}^{\mathrm{r}}$ is the $j$-th row of $\mathbf{W}$. Since $\mathbf{W}$ is symmetric, $\left(\mathbf{w}_{j}^{\mathrm{r}}\right)^{\mathrm{T}}=\mathbf{w}_{j}$.

The matrix $D$ in (2.8) is a diagonal matrix, and

$$
\begin{aligned}
D & =\frac{1}{\lambda} \sum_{k=1}^{m} W_{l_{k}}^{\mathrm{D}}+\operatorname{diag}\left\{2 \sum_{j=1}^{N} \mathbf{w}_{j}\right\} \\
W_{s} & =2 \mathbf{W}
\end{aligned}
$$

To minimize the cost function $J$, we take the derivative of (2.7) with respective to $\mathbf{v}$ and set it as zero, i.e.,

$$
\frac{\mathrm{d} J}{\mathrm{~d} \mathbf{v}}=2(H \mathbf{v}-\mathbf{b})=0
$$

From (2.9), we can easily obtain

$$
\hat{\mathbf{v}}=H^{-1} \mathbf{b}=\left(D-W_{s}\right)^{-1} \mathbf{b}
$$

Substituting $\hat{\mathbf{v}}$ into (2.5), we obtain the calibrated physical field

$$
f\left(\mathbf{y}_{i}\right)=f_{c}\left(\mathbf{y}_{i}\right)-\hat{v}_{i}, \quad \mathbf{y}_{i} \in \Omega_{\mathrm{D}} \text { and } 1 \leq i \leq N
$$

For the matrix $H \in \mathbb{R}^{N \times N}, N$ is the number of mesh points. Generally, $N$ is very large and therefore, the computational cost of solving $H^{-1}$ in (2.10) is extremely 
expensive. In practice, we cannot directly obtain $\hat{\mathbf{v}}$ from $(2.10)$. Thus, we provide a computationally efficient method to find the approximated solution of $\hat{\mathbf{v}}$.

\subsection{Computational efficient approximated solu- tion}

To avoid directly solving the inverse of the matrix $H$, which has a very large dimension, we provide an approximated solution of the inverse problem. A similar method has been previously applied for image editing [81].

Considering the definition of $w_{i j}$, i.e., $w_{i j}=w\left(\mathbf{y}_{i}, \mathbf{y}_{j}\right)$, we find that each column of W represents the affinities between the point ( whose mesh index is the column index) and the whole physical field. The neighbouring points have similar affinities with other points. Hence, the neighbouring columns have similar entries, especially for the entries far away from the diagonal, which implies that the neighbouring columns of $\mathbf{W}$ have a large correlation coefficient. Therefore, the matrix $\mathbf{W}$ has many near zero eigenvalues, and it can be approximated by a low rank matrix.

Since the matrix $W_{s}=2 \mathbf{W} \in \mathbb{R}^{N \times N}, W_{s}$ can also be approximated by a low rank matrix. We rewrite the symmetric matrix $W_{s}$ as

$$
W_{s}=\left[\begin{array}{cc}
A & B^{T} \\
B & C
\end{array}\right]
$$

where $A \in \mathbb{R}^{n \times n}, B \in \mathbb{R}^{(N-n) \times n}$, and $C \in \mathbb{R}^{(N-n) \times(N-n)}$.

We assume that the number of dominant eigenvalues of $W_{s}$ is $n$. Using the eigen decomposition and ignoring all the near zero eigenvalues, we can approximate $W_{s}$ as

$$
W_{s}=U \Lambda U^{\mathrm{T}} \approx U\left[\begin{array}{cc}
\Lambda_{1} & \mathbf{0} \\
\mathbf{0} & \mathbf{0}
\end{array}\right] U^{\mathrm{T}}=V V^{\mathrm{T}}
$$


where $U$ is an orthogonal matrix, $\Lambda$ is a diagonal matrix with all eigenvalues of $W_{s}$ in the diagonal, $\Lambda_{1}=\Lambda(1: n, 1: n)$ consists of all dominant eigenvalues of $W_{s}$ in its diagonal, and $V \in \mathbb{R}^{N \times n}$ has exactly rank $n$. We denote $V$ by

$$
V=\left[\begin{array}{l}
X \\
Y
\end{array}\right]
$$

where $X$ and $Y$ have the same sizes as $A$ and $B$ in (2.12), respectively. Then, we can obtain that

$$
W_{s} \approx\left[\begin{array}{c}
X \\
Y
\end{array}\right]\left[\begin{array}{ll}
X^{\mathrm{T}} & Y^{\mathrm{T}}
\end{array}\right]=\left[\begin{array}{cc}
X X^{\mathrm{T}} & X Y^{\mathrm{T}} \\
Y X^{\mathrm{T}} & Y Y^{\mathrm{T}}
\end{array}\right]
$$

As illustrated in Figure 2.1, which was firstly used in [81], comparing (2.13) and (2.12), we find

$$
C \approx Y Y^{\mathrm{T}}=Y X^{\mathrm{T}}\left(\left(X^{\mathrm{T}}\right)^{-1} X^{-1}\right) X Y^{\mathrm{T}}=B A^{-1} B^{\mathrm{T}}
$$
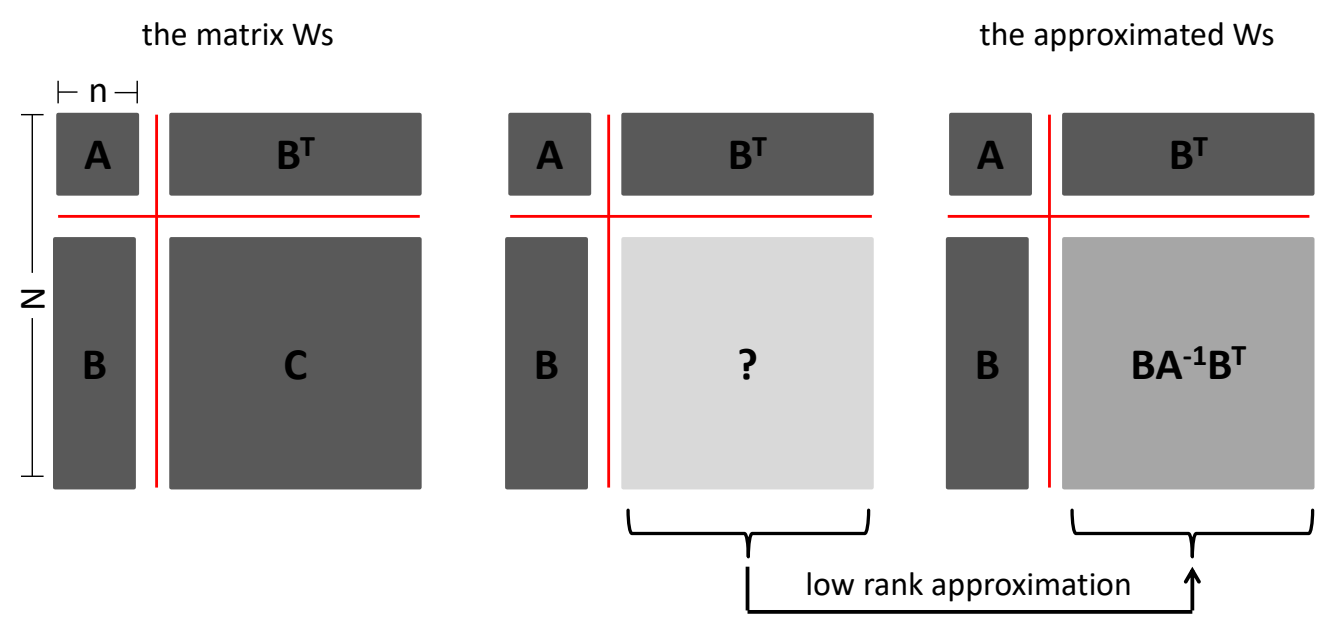

FIgURE 2.1: Illustration of the low rank approximation of $W_{s}$.

Substituting (2.14) into (2.12) yields

$$
W_{s} \approx\left[\begin{array}{cc}
A & B^{\mathrm{T}} \\
B & B A^{-1} B^{\mathrm{T}}
\end{array}\right]=\left[\begin{array}{l}
A \\
B
\end{array}\right] A^{-1}\left[\begin{array}{cc}
A & B^{\mathrm{T}}
\end{array}\right]=Z A^{-1} Z^{\mathrm{T}}
$$


where $Z=\left[\begin{array}{l}A \\ B\end{array}\right]$ is the first $n$ column of $W_{s}$. Using this way, we can find a low rank approximation of $W_{s}$, which can both resolve the storage and computational cost problems in solving $H^{-1}$.

Substituting the approximation of $W_{s}$ in (2.15) into (2.8) and using the Woodbury formula [83], we can obtain

$$
H^{-1} \approx\left(D-Z A^{-1} Z^{\mathrm{T}}\right)^{-1}=D^{-1}-D^{-1} Z\left(-A+Z^{T} D^{-1} Z\right)^{-1} Z^{T} D^{-1}
$$

Then, substituting (2.16) into (2.10) yields

$$
\hat{\mathbf{v}}=D^{-1} \mathbf{b}-D^{-1} Z\left(-A+Z^{\mathrm{T}} D^{-1} Z\right)^{-1} Z^{\mathrm{T}} D^{-1} \mathbf{b}
$$

In (2.17), we need to solve the inverse of two matrices. $D$ is a diagonal matrix and its inverse can be easily solved. Because $\left(-A+Z^{\mathrm{T}} D^{-1} Z\right) \in \mathbb{R}^{n \times n}$ and $n \ll N$, solving $\left(-A+Z^{\mathrm{T}} D^{-1} Z\right)^{-1}$ is computationally much more efficient.

Substituting (2.17) into (2.11), we can obtain the calibrated physical field. Next, we show an experiment to support the proposed methodology.

\subsection{Experimental verification: indoor thermal map calibration}

\subsubsection{Statement of the experiment}

We tested the proposed methodology for a $7.28 m \times 3.32 m \times 2.5 m$ room in the School of Electrical and Electronic Engineering, Nanyang Technological University, which is shown in Figure 2.2. An Active Chilled Beam system (ACBs) was installed for air conditioning, as shown on the ceiling of the room in Figure 2.2(A). One air outlet is at the corner of the room. The walls and the door are made by thermal insulation 
materials, and the windows are double glazing. Hence, in the CFD simulation, we simply set the walls, the door, and the windows as insulated walls.

As shown in Figure 2.2, the room contains four heaters $(0.76 m \times 1.2 m \times 0.02 m)$. As shown in Figure 2.2(B), the left two heaters are 300W, and the other two are $400 \mathrm{~W}$. The height above the ground of the top surfaces of the heaters is $1.02 \mathrm{~m}$. The bottom faces of the heaters were made by insulated materials. One sensor was used to measure the velocity of the inlet cooling air, as shown in Figure 2.3(A). Another sensor was used to measure the inlet cooling air temperature, which is shown in Figure 2.3(B). The other eight sensors were used to measure the temperature of a horizontal plane $1.2 \mathrm{~m}$ above the ground. Four sensors were hung and suspended just above centers of the four heaters, respectively. The four sensors are represented by stars (s1-s4) in Figure 2.2(B). One of the four is shown in Figure 2.3(C). Three sensors (s5-s7) were placed as the middle plane of the room. The sensor s8 was placed $20 \mathrm{~cm}$ from the wall. All the eight sensors (s1-s8) were $1.2 \mathrm{~m}$ above the ground.

The experiment was carried out on 26th May, 2016. The four heaters and the air-condition system were turned on at 9:30 am. We collected the velocity and temperature of the inlet cooling air (via the sensors $\mathrm{s}_{v}$ and $\mathrm{s}_{t}$, respectively) from 11:17 to 13:10. The data is shown in Figure 2.4. The sensors s1-s7 recorded the temperature of the $1.2 \mathrm{~m}$ plane from 9:30 to $13: 10$, the data for which is shown in Figure 2.5. The sensor s8 cannot store the sensor readings. We have recorded that after 11:00, the readings of sensor s8 remained at $25.6^{\circ} \mathrm{C}$.

\subsubsection{CFD simulation}

The simulation was performed using the commercial CFD software Ansys FLUENT v.13.0. The proposed calibration methodology has the potential to be applied for dynamic CFD simulations if both the real-time simulation results and sensor observations are available. In this work, however, for simplicity we only consider the steady state simulation results. 


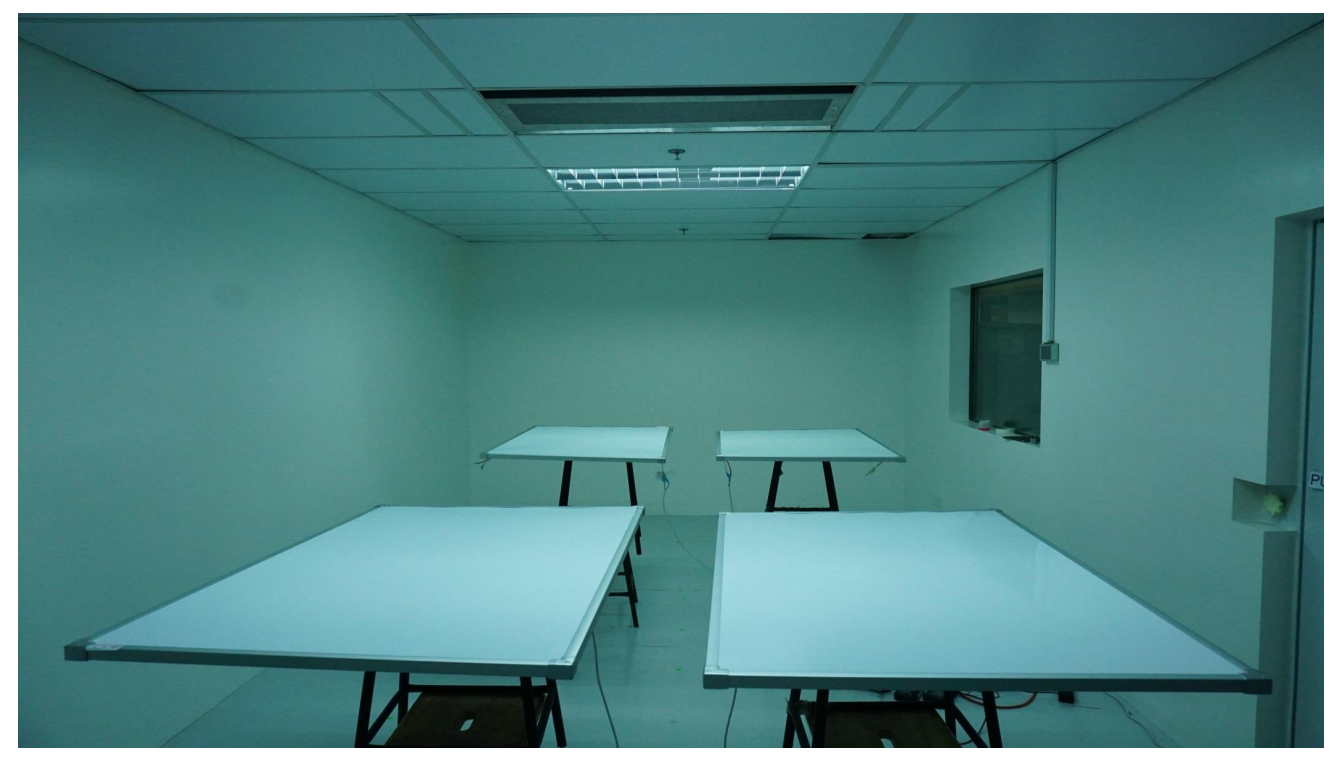

(A) Room layout

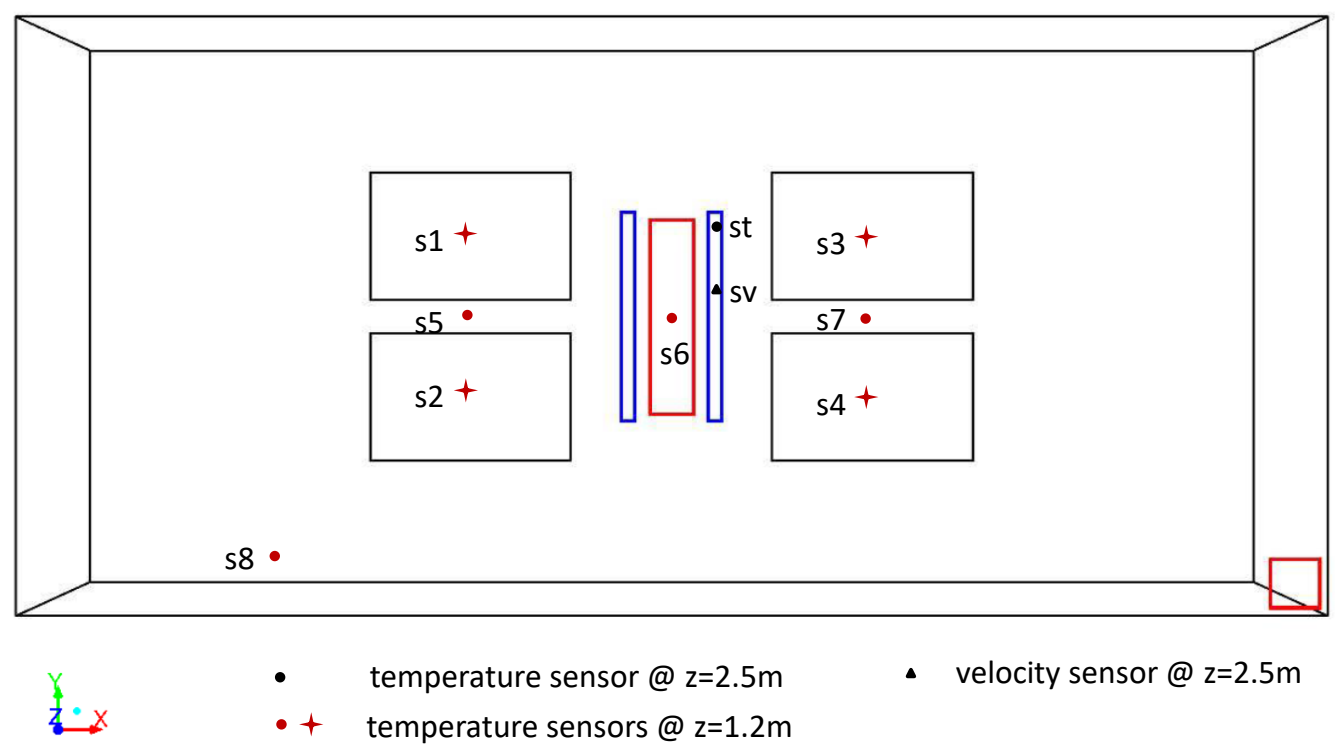

(B) Top view sketch map

Figure 2.2: The test bed: a $7.28 \mathrm{~m} \times 3.32 \mathrm{~m} \times 2.5 \mathrm{~m}$ room located at the Process Control and Instrumentation Lab in Nanyang Technological University, Singapore. The bottom figure is the top view sketch map. The red square and the red rectangle are the two outlets. The center one is for induced air, and the corner one is for exhaust air. The two blue rectangles represent the two cooling air inlets. The black triangle represents an air velocity sensor, as shown in Figure 2.3(A). The black dot represents a temperature sensor as shown in Figure 2.3(B). The four red stars and four red dots represent eight temperature sensors located $1.2 \mathrm{~m}$ above the ground. The four black squares are four heaters. The hight of the heaters were $1 \mathrm{~m}$ above the ground. 


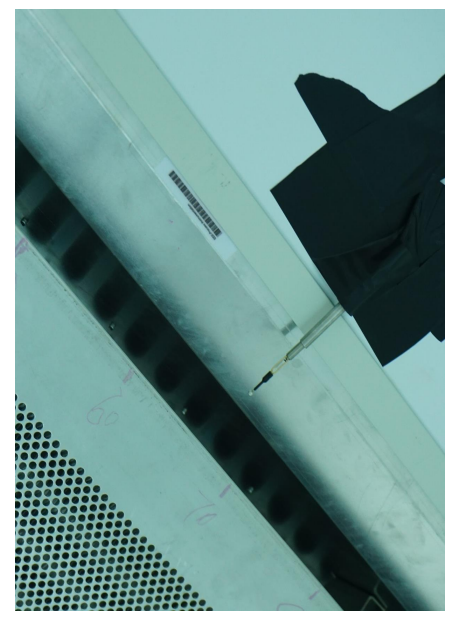

(A)

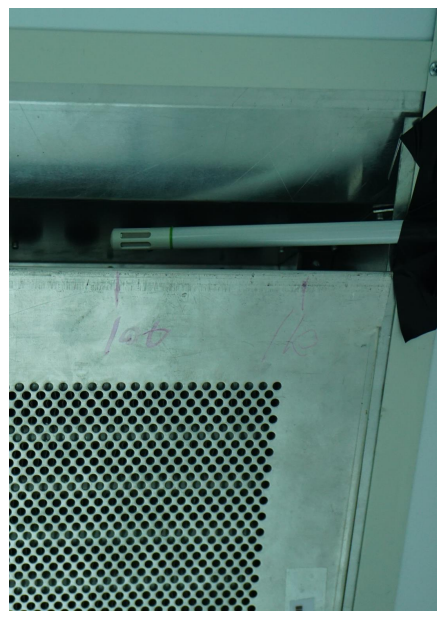

(в)

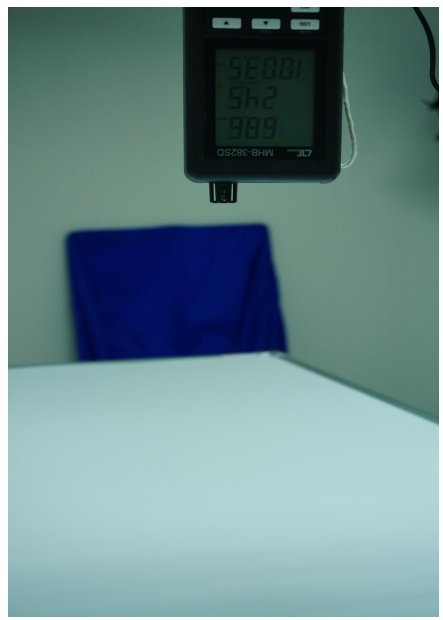

(c)

FiguRE 2.3: Sensors used for indoor parameters measurement. (A): velocity sensor used to measure the inlet air velocity. (B): temperature sensor used to measure the inlet cooling air temperature. (C): temperature sensor used to measure the temperature at $1.2 \mathrm{~m}$ hight, which is hung above the heater.

The indoor air was assumed as the ideal gas with density of $1.225 \mathrm{~kg} / \mathrm{m}^{3}$. The viscous model was chosen as the realizable $k-\varepsilon$ model. Since the test-bed was built in a laboratory, we did not consider the solar radiation. The door, ground, ceiling, windows, and walls are assumed to be insulated walls. During the experiment, all lights were turned off. For the velocity and temperature of the inlet cooling air, we use the mean values of the sensor readings in the time period 12:10 to 13:10 in Figure 2.4. The bottom faces of the heaters were made by insulated material, but they were not completely insulated. We assumed that $5 \%$ of energy was transferred via the bottom faces of the heaters. Corresponding to the four sensors s1-s4, we denote the four heaters by heater 1 , heater 2 , heater 3 and heater 4 , respectively. The boundary conditions of the CFD simulation are detailed in Table 2.1.

We created the structured mesh using Gambit v2.4. Since all the walls are insulated, we did not consider the boundary layer of the walls and uniformly divided the horizontal plane $(X-Y$ plane $)$ into $2 \mathrm{~cm} \times 2 \mathrm{~cm}$ squares. In the $z$-direction, we made the mesh dense near the ceiling $(z=2.5 \mathrm{~m})$ and heater top faces $(z=1.02 \mathrm{~m})$, and divided the entire $2.5 \mathrm{~m}$ height into 137 intervals. The total mesh size is $\Delta_{1}=167 \times 365 \times 137-4 \times 37 \times 59 \times 1=8,342,103$. 


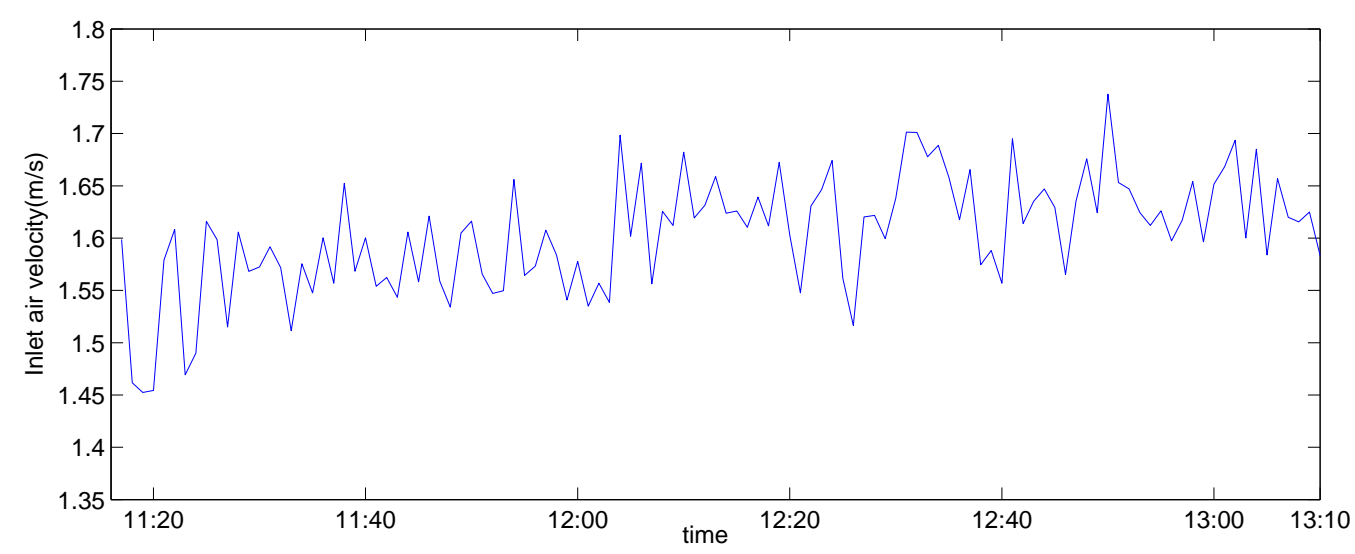

(A) inlet air velocity

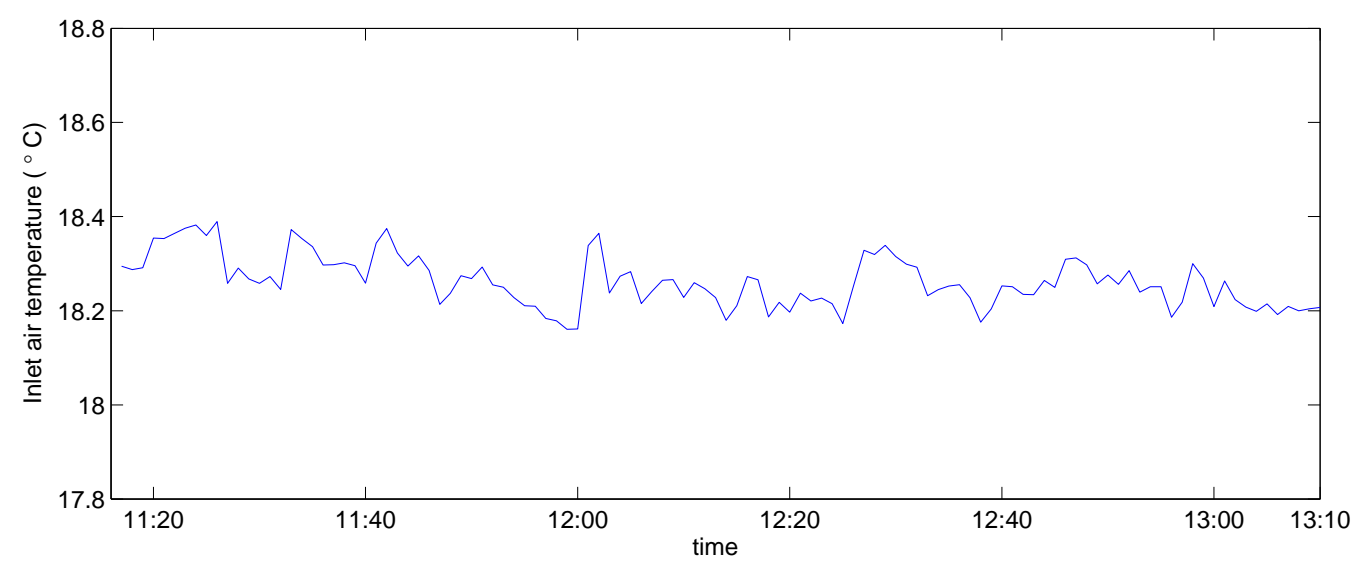

(в) inlet air temperature

Figure 2.4: The recorded inlet cooling air velocity and temperature during 11:17 to $13: 10$ on 26th May, 2016.

To verify the grid independency, we made the mesh dense and increased the mesh size to $\Delta_{2}=18,527,205$. We did CFD simulations for both mesh files. The results were found to be in good agreement. Therefore, the mesh file with 8,342,103 cells is chosen to reduce computation time.

The convergence criteria were set as $10^{-6}$ for energy and $10^{-3}$ for all the other parameters. As shown in Figure 2.6, the simulation converged with 442 iterations. We are interested in the thermal map of the room at $1.2 \mathrm{~m}$ height. The thermal map obtained from the CFD simulation is shown in Figure 2.7(A). 


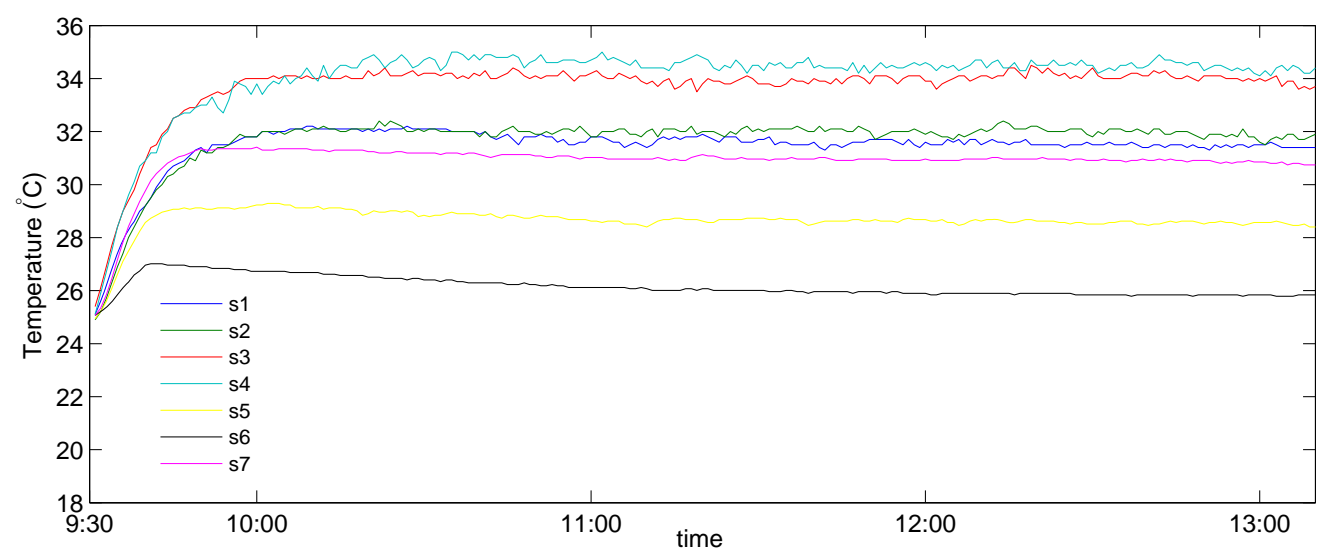

FiguRE 2.5: The temperature recording of the seven sensors (s1-s7).

TABLE 2.1: Boundary conditions of the CFD simulation

\begin{tabular}{|c|c|c|c|}
\hline Boundary & Type & Heat transfer & Mass \& momentum \\
\hline The two inlets & velocity-inlet & $T_{i}=18.24^{\circ} \mathrm{C}$ & $\begin{array}{l}V_{\text {in }}=1.62 \mathrm{~m} / \mathrm{s} \\
\theta=25^{\circ}\end{array}$ \\
\hline Center outlet & pressure-outlet & $T_{o}=25^{\circ} \mathrm{C}$ & $P_{\text {relative }}=-10 \mathrm{~Pa}$ \\
\hline Corner outlet & pressure-outlet & $T_{o}=25^{\circ} \mathrm{C}$ & $P_{\text {relative }}=0 \mathrm{~Pa}$ \\
\hline $\begin{array}{l}\text { Walls, windows, } \\
\text { ceiling \& ground }\end{array}$ & wall & Insulated & No slip wall \\
\hline $\begin{array}{l}\text { Top \& side faces } \\
\text { (heaters } 1 \& 2 \text { ) }\end{array}$ & wall & $h_{c}=287.8 \mathrm{~W} / \mathrm{m}^{2}$ & No slip wall \\
\hline $\begin{array}{l}\text { Top \& side faces } \\
\text { (heaters } 3 \& 4 \text { ) }\end{array}$ & wall & $h_{c}=383.7 \mathrm{~W} / \mathrm{m}^{2}$ & No slip wall \\
\hline $\begin{array}{l}\text { Bottom faces } \\
\text { (heaters } 1 \& 2 \text { ) }\end{array}$ & wall & $h_{c}=16.4 \mathrm{~W} / \mathrm{m}^{2}$ & No slip wall \\
\hline $\begin{array}{l}\text { Bottom faces } \\
\text { (heaters } 3 \& 4 \text { ) }\end{array}$ & wall & $h_{c}=21.9 \mathrm{~W} / \mathrm{m}^{2}$ & No slip wall \\
\hline
\end{tabular}

$T_{i}-$ inlet cooling air temperature; $V_{\text {in }}-$ inlet cooling air speed; $\theta-$ the angle of $V_{\text {in }}$ with the ceiling; $T_{o}-$ outlet air temperature; $P_{\text {relative }}-$ pressure relative to the reference pressure $\left(P_{\text {reference }}=101325 \mathrm{~Pa}\right) ; h_{c}-$ convective heat flux.

\subsubsection{The calibration results}

We use the sensor observations obtained from sensors s1-s4 to calibrate the thermal map in Figure 2.7(A). The four sensors s1-s4 were just above the centers of the four heaters, respectively. We set $\sigma_{m}=1000$ and $\sigma_{d}=1$.

The thermal map in Figure 2.7(A) consists of $60955(=167 \times 365)$ temperature 


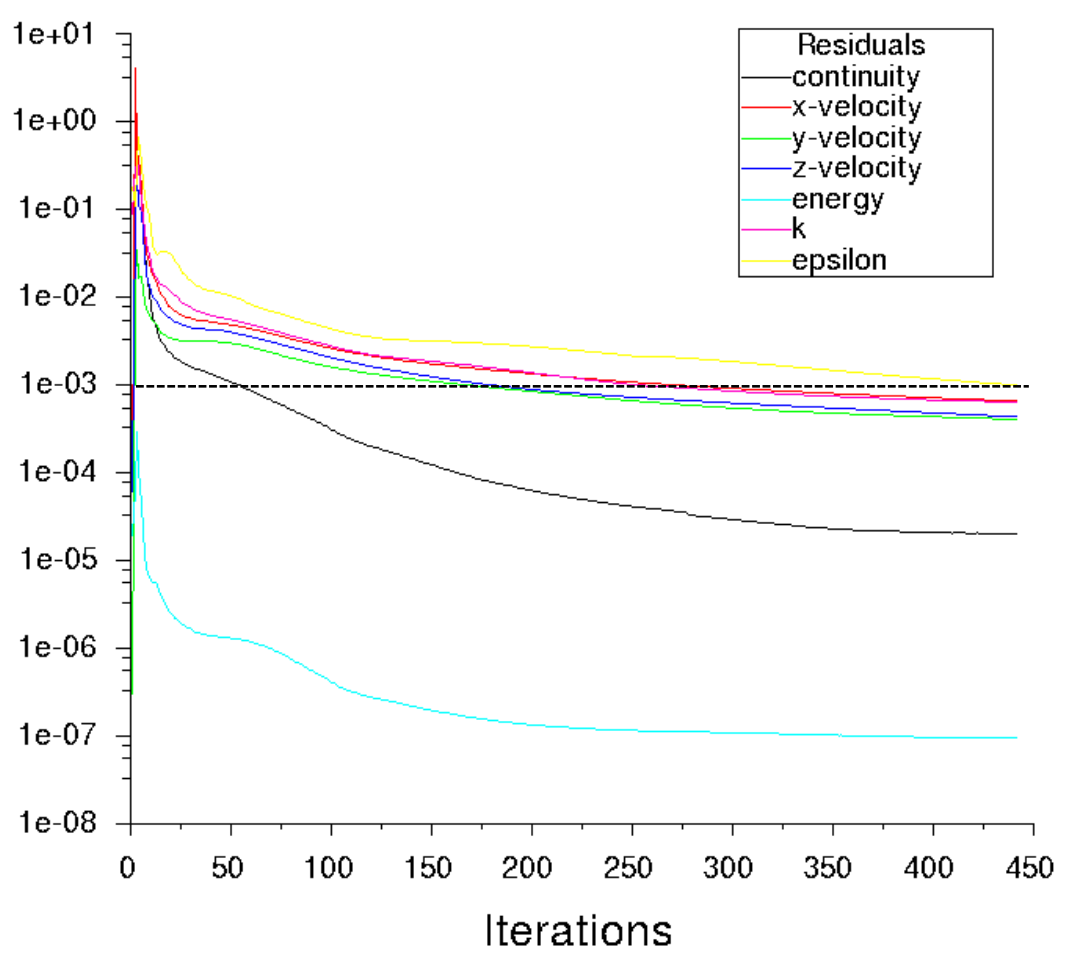

FiguRE 2.6: Convergence history of the residuals.

values of all the mesh points at the plane $z=1.2 \mathrm{~m}$. The balance factor $\lambda$ is related to the number of mesh points and the number of sensor observations. We set

$$
\lambda=\alpha \frac{m}{N}=\frac{4 \alpha}{60955}
$$

where $0<\alpha \leq 1$. The first part in (2.6) is the summation of $m \times N$ terms while the second part is the summation of $N \times N$ terms. If we set $\alpha=1$, it means that we have the same trust on the sensor observations and the simulation results on all mesh points. Obviously, sensor observations are more accurate. We set $\alpha=0.01$ and the calibrated thermal map is shown in Figure 2.7(B). Here, $\alpha=0.01$ implies that the influence of each sensor observation in (2.6) is 100 times important than the simulated data on each mesh point.

Comparing Figure 2.7(B) with Figure 2.7(A), we can easily find that the calibrated thermal map preserves the profile of the CFD results. However, Figure2.7(B) cannot show how the simulated thermal map is calibrated. We show the estimated error (i.e., $\hat{\mathbf{v}}$ ) in Figure 2.8(A), which is the difference between Figure 2.7(A) and 


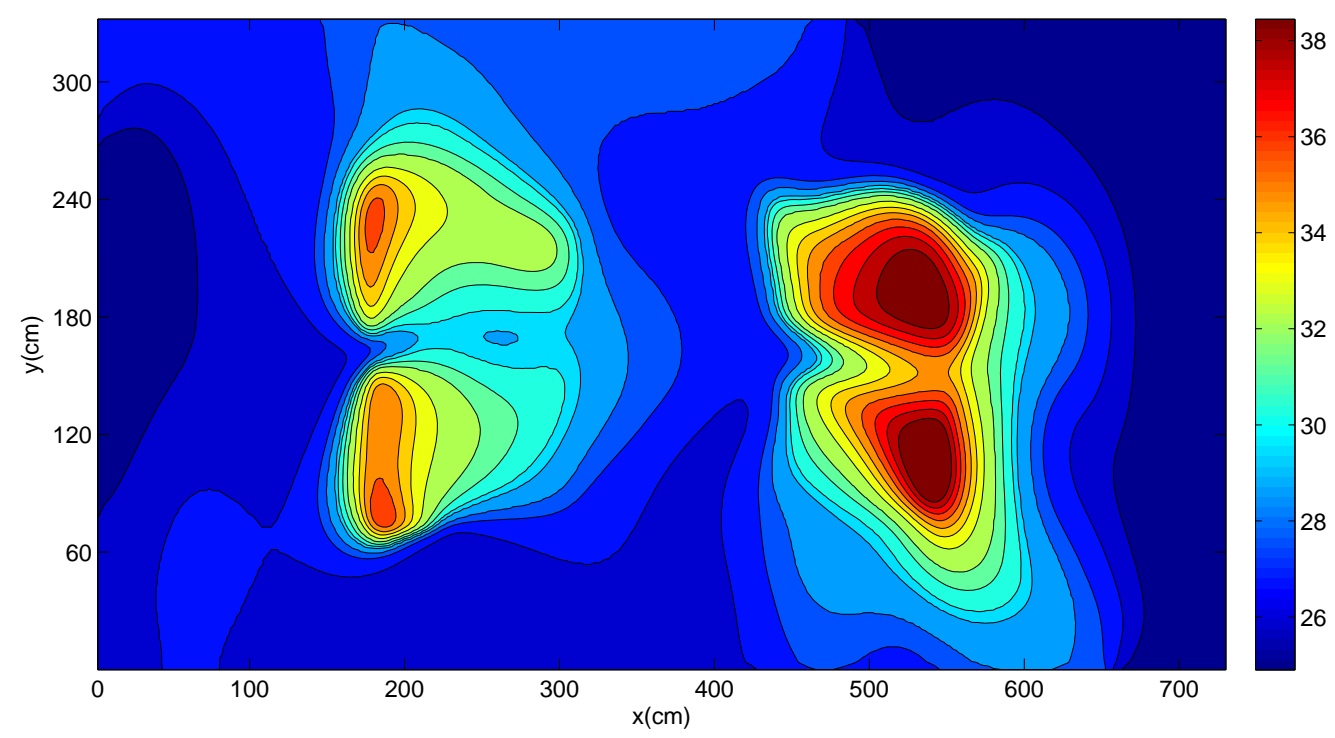

(A) simulated thermal map

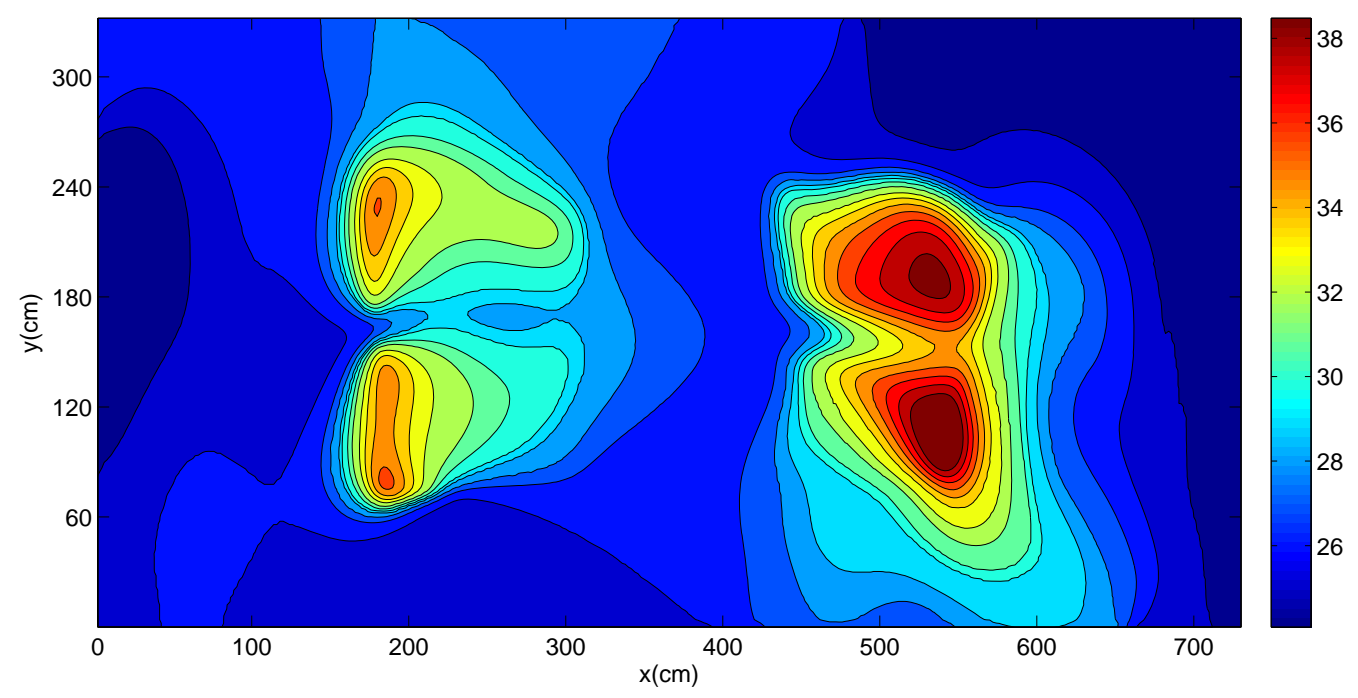

(B) calibrated thermal map with $\alpha=0.01, \sigma_{m}=1000$ and $\sigma_{d}=1$

Figure 2.7: The thermal map (temperature field in ${ }^{\circ} \mathrm{C}$ ) $1.2 \mathrm{~m}$ above the ground.

Figure 2.7(B). Considering (2.11), we can obtain the simulated thermal map by subtracting the estimated error from the simulated thermal map.

\subsubsection{The influence of balance factor}

To check the effect of the balance factor, we set $\alpha$ as $0.01,0.1,0.5$, and 1 respectively. The corresponding estimated errors of the thermal map in Figure 2.7(A) is 


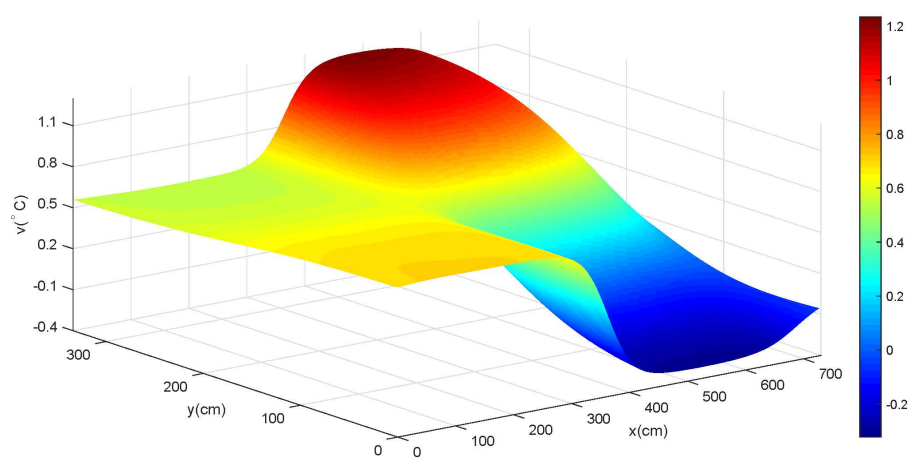

(A) $\alpha=0.01$

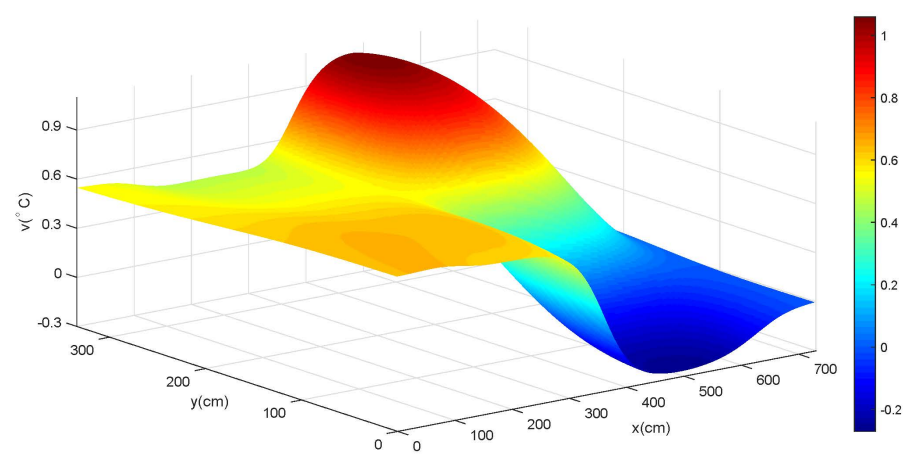

(B) $\alpha=0.1$

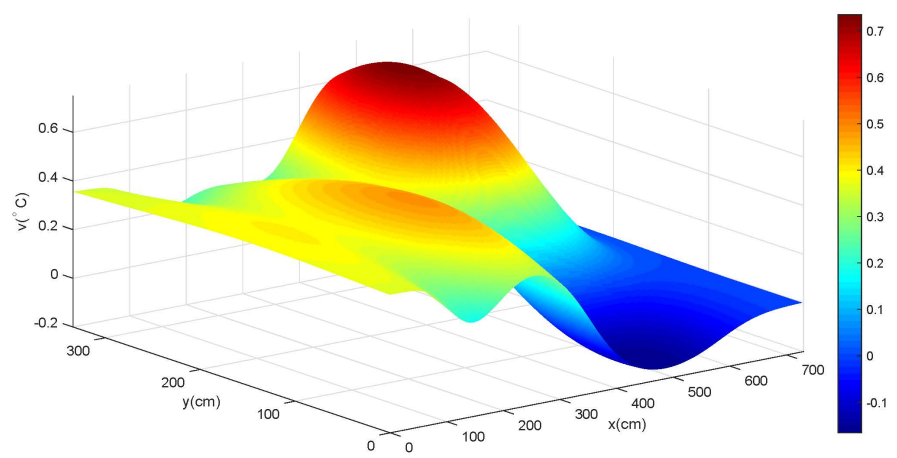

(C) $\alpha=0.5$

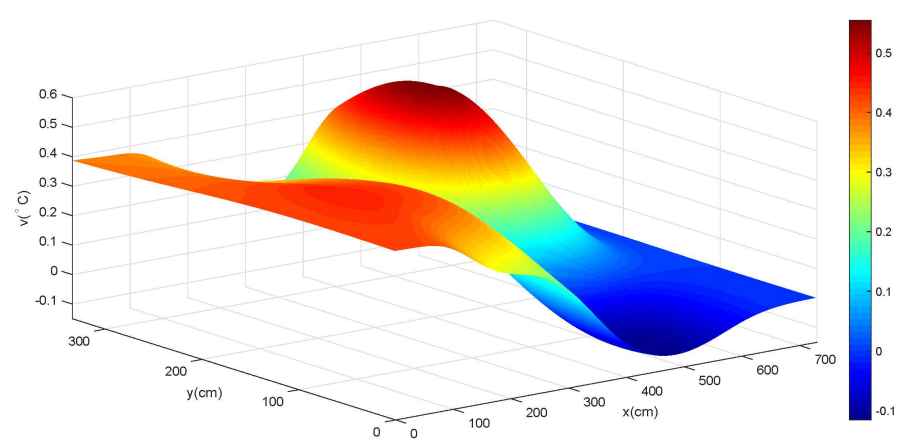

(D) $\alpha=1$

FiguRE 2.8: The estimated error $(\hat{\mathbf{v}})$ of the thermal map in Figure 2.7(A) w.r.t. different balance factor. $\sigma_{m}=1000$ and $\sigma_{d}=1$. 
given in Fig 2.8. The observations of sensors s1-s4 are used to estimate the error. To show the effectiveness of the proposed method, we use the observations of sensor s5-s8 as the ground truth to test the estimation performance. The error of the CFD simulation results and the calibrated thermal map at the 8 sensing locations are shown in Figure 2.9.

Figure 2.8 shows that with different balance factors, the estimated errors (i.e., $\hat{\mathbf{v}}$ ) almost have the same profiles. However, the magnitude of the estimated errors decrease when the balance factor increases, which implies that the influence of sensor observations decreases and we can only provide a very small adjustment for the original simulated thermal map. In other words, the balance factor can control the magnitude of the estimated errors, i.e., the magnitude of the adjustment for the simulated thermal map. As shown in Figure 2.8 and Figure 2.9, with a smaller balance factor, the magnitude of the estimated error is larger and we can obtain a better performance.

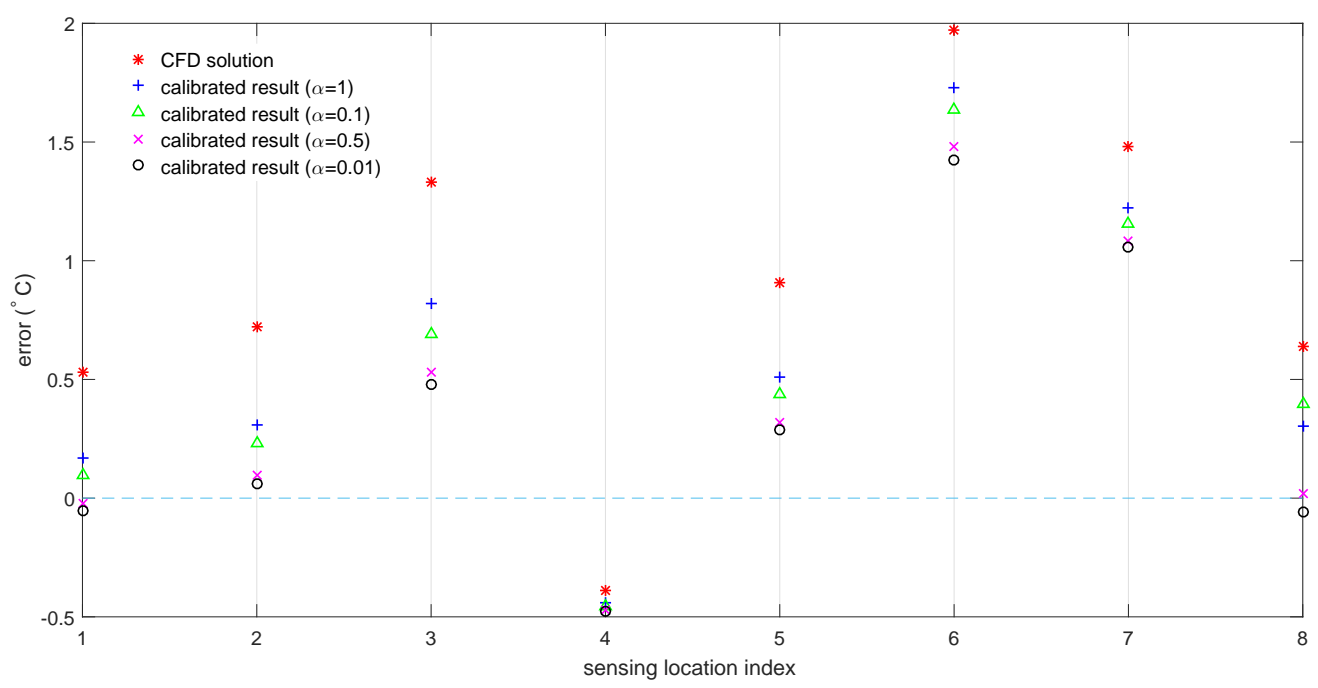

Figure 2.9: The errors of CFD solution and the calibrated thermal maps at the sensing locations s1-s8 with $\sigma_{m}=1000$ and $\sigma_{d}=1$

The root mean square error (RMSE) of the thermal map data at sensing locations are given in Table 2.2. Both Figure 2.9 and Table 2.2 show that we can enhance the thermal map obtained from CFD simulation with the four sensor observations (s1-s4). With the calibration, the RMSE of the data at sensing locations s5-s8 can 
TABLE 2.2: The RMSE of the thermal maps data at the sensing locations w.r.t. different balance factor

\begin{tabular}{l|cc|cc}
\hline \hline Data at the sensing locations & $\begin{array}{c}\text { RMSE } \\
(\mathrm{s} 1-\mathrm{s} 8)\end{array}$ & $\begin{array}{c}\text { improvement } \\
(\mathrm{s} 1-\mathrm{s} 8)\end{array}$ & $\begin{array}{c}\text { RMSE } \\
(\mathrm{s} 5-\mathrm{s} 8)\end{array}$ & $\begin{array}{c}\text { improvement } \\
(\mathrm{s} 5-\mathrm{s} 8)\end{array}$ \\
\hline CFD simulation result & 1.1197 & $/$ & 1.3518 & $/$ \\
calibrated result $(\alpha=1)$ & 0.8536 & $23.77 \%$ & 1.0999 & $18.55 \%$ \\
calibrated result $(\alpha=0.5)$ & 0.7996 & $28.59 \%$ & 1.0451 & $22.59 \%$ \\
calibrated result $(\alpha=0.1)$ & 0.7045 & $37.08 \%$ & 0.9301 & $31.06 \%$ \\
calibrated result $(\alpha=0.01)$ & 0.6794 & $39.32 \%$ & 0.8985 & $33.38 \%$ \\
\hline \hline
\end{tabular}

Note: Both $\sigma_{m}$ and $\sigma_{d}$ are fixed. We set $\sigma_{m}=1000$ and $\sigma_{d}=1$. Here, improvement $=(\mathrm{RMSE}$ of CFD simulation result $-\mathrm{RMSE}$ of calibrated result $) / \mathrm{RMSE}$ of CFD simulation result.

be improved by $33.38 \%$. However, we can see from Figure 2.9 that the errors of the regions around $\mathrm{s} 6$ and $\mathrm{s} 7$ are still large.

The CFD simulation results around s6 and s7 have large errors, i.e., around $2^{\circ} \mathrm{C}$ and $1.5^{\circ} \mathrm{C}$, respectively. The proposed method reduces the errors to around $1.5^{\circ} \mathrm{C}$ and $1^{\circ} \mathrm{C}$, which are still large. To address this issue, we should consider the capacity of the proposed method. The largest magnitude to which the proposed method can adjust the CFD results almost equals the maximum error of the CFD results at all sensing locations where the sensor observations were used to calibrate the CFD results. It can be seen in Figure 2.9 that the maximum error of the CFD results at sensing locations s1-s4 is around $1.3^{\circ} \mathrm{C}$. Therefore, the maximum value of the estimated error of the simulated thermal map is less than $1.3^{\circ} \mathrm{C}$. The balance factor can control the magnitude of the maximum value of the estimated errors. In other words, the balance factor can control the magnitude of the adjustment for the simulated thermal map, but the upper bound of this magnitude is determined by the sensing locations. 


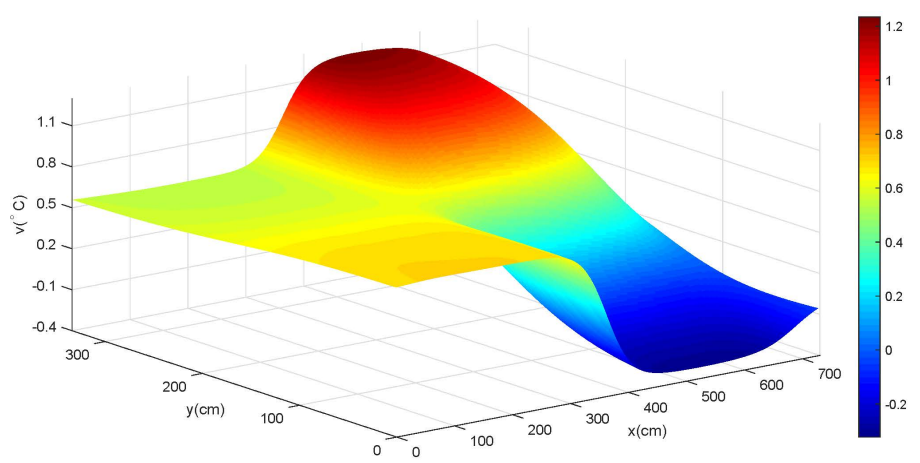

(A) $\sigma_{m}=1000$

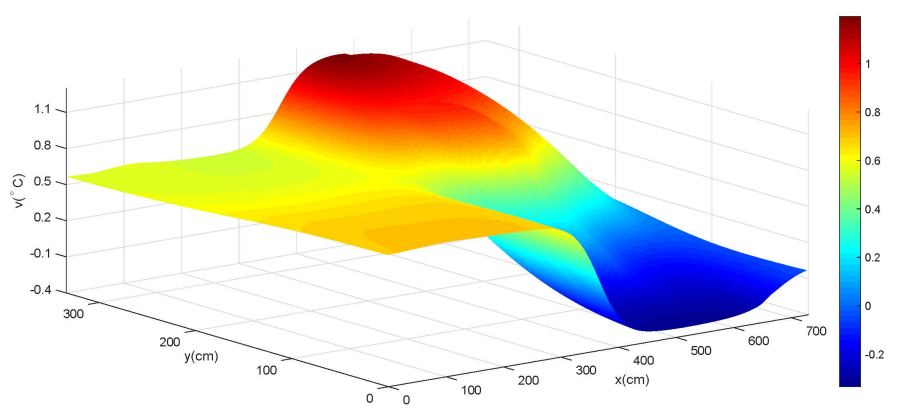

(в) $\sigma_{m}=100$

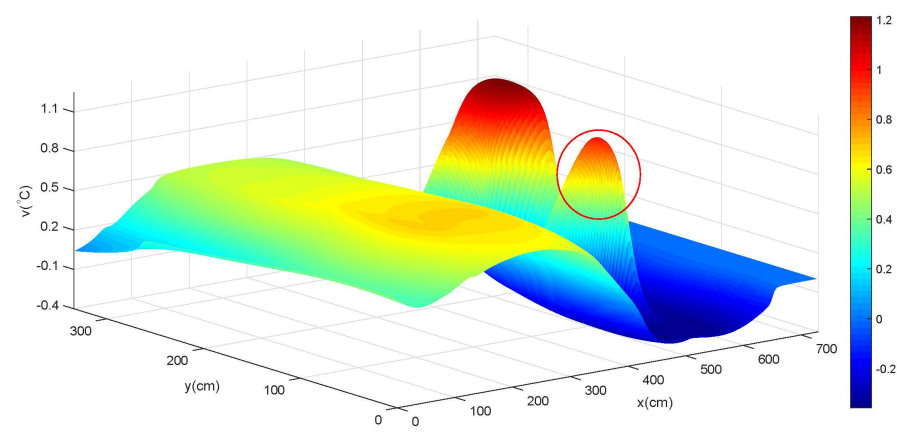

(C) $\sigma_{m}=10$

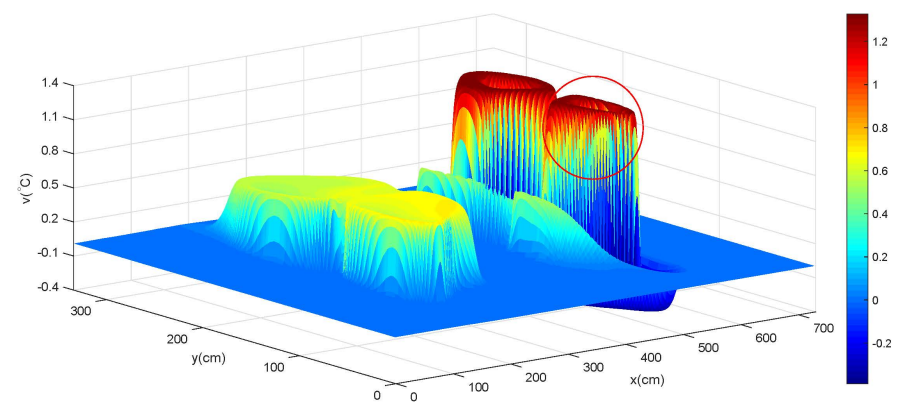

(D) $\sigma_{m}=1$

Figure 2.10: The estimated error $(\hat{\mathbf{v}})$ of the thermal map in Figure 2.7(A) w.r.t. different magnitude variance $\sigma_{m} . \alpha=0.01$ and $\sigma_{d}=1$. 


\subsubsection{The influence of magnitude variance}

To check the influence of magnitude variance, we set $\sigma_{m}$ as $1000,100,10$, and 1 , respectively. With the four sensor observations (s1-s4), we estimated the error of the thermal map in Figure 2.7(A). The estimated errors are given in Figure2.10. The error of the estimated thermal map at the 8 sensing locations are shown in Figure 2.11, and the RMSE of the calibrated thermal map at the 8 sensing locations are given in Table 2.3.

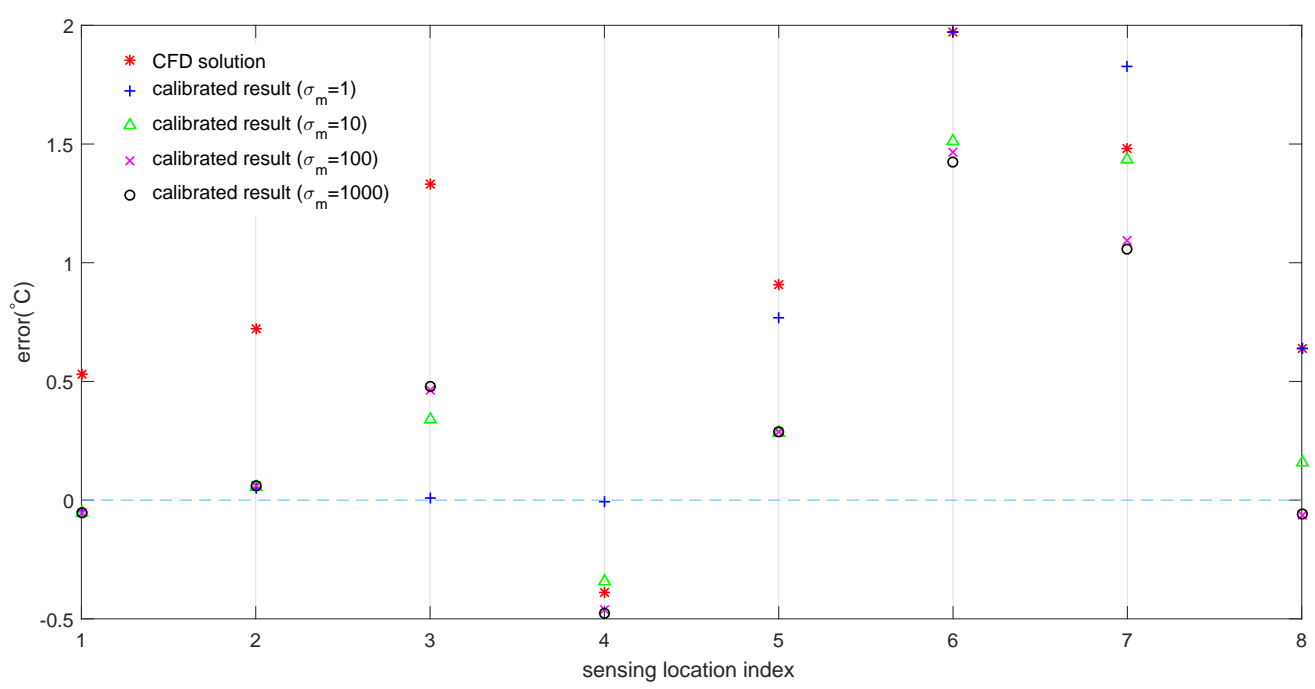

Figure 2.11: The errors of CFD simulation result and the calibrated thermal maps at the sensing locations s1-s8 with $\alpha=0.01$ and $\sigma_{d}=1$

Figure 2.10 shows that with different magnitude variances, the maximum magnitude of the estimated errors (i.e., $\hat{\mathbf{v}}$ ) are almost the same, which implies that different with the balance factor, the magnitude variance has no significant influence on the the maximum magnitude of the estimated errors.

It can be seen from Figure 2.10(C-D) that the region in the two red circle is nearer to sensor s4 than s3 but the estimated error is similar with the estimated error around s3. We can see form Figure 2.7(A) that the temperature values of the thermal map in the region marked by the two circles (in Figure 2.10(C-D)) are similar to those in the region around sensor s3. The proposed thermal map calibration work is a process of fusing sensor information and the information of 
CFD results. A smaller magnitude variance can enhance the influence of the CFD results in this information fusion work.

In addition, Figure 2.10(D) shows that if $\sigma_{m}=1$ in the regions far away the sensing locations s1-s4, the estimated errors are almost zero. In other words, the proposed method has no influence for these regions. As we mentioned earlier, a small magnitude variance leads to a small value of the first Gaussian function in (2.4), which can dilute the influence of the second Gaussian function. The smaller the magnitude variance $\sigma_{m}$, the smaller the weight $w\left(\mathbf{x}_{k}, \mathbf{y}\right)$, especially when $\mathbf{y}$ is far away the sensing location $\mathbf{x}_{k}$. Therefore, if the magnitude variance is too small, the proposed method could not globally calibrate the thermal map.

TABLE 2.3: The RMSE of the thermal maps data at the sensing locations w.r.t. different magnitude variance $\sigma_{m}$

\begin{tabular}{l|cc|cc}
\hline \hline Data at the sensing locations & $\begin{array}{c}\text { RMSE } \\
(\mathrm{s} 1-\mathrm{s} 8)\end{array}$ & $\begin{array}{c}\text { improvement } \\
(\mathrm{s} 1-\mathrm{s} 8)\end{array}$ & $\begin{array}{c}\text { RMSE } \\
(\mathrm{s} 5-\mathrm{s} 8)\end{array}$ & $\begin{array}{c}\text { improvement } \\
(\mathrm{s} 5-\mathrm{s} 8)\end{array}$ \\
\hline CFD simulation result & 1.1197 & $/$ & 1.3518 & $/$ \\
calibrated result $\left(\sigma_{m}=1\right)$ & 1.0142 & $9.42 \%$ & 1.4339 & $-6.05 \%$ \\
calibrated result $\left(\sigma_{m}=10\right)$ & 0.7652 & $31.66 \%$ & 1.0543 & $21.91 \%$ \\
calibrated result $\left(\sigma_{m}=100\right)$ & 0.6957 & $37.87 \%$ & 0.9267 & $31.31 \%$ \\
calibrated result $\left(\sigma_{m}=1000\right)$ & 0.6794 & $39.32 \%$ & 0.8985 & $33.38 \%$ \\
\hline \hline
\end{tabular}

Note: Both $\alpha$ and $\sigma_{d}$ are fixed. We set $\alpha=0.01$ and $\sigma_{d}=1$. Here, improvement $=(\mathrm{RMSE}$ of CFD simulation result $-\mathrm{RMSE}$ of calibrated result)/RMSE of CFD simulation result.

Figure 2.11 shows that with the decrease of the magnitude variance, the errors around sensing locations s1-s4 also decrease but those around sensing locations s5s8 do not. From Table 2.3, we can find that the RMSE at sensing locations s5-s8 increases with respect to the decrease of the magnitude variance. If we reduce the magnitude variance to 1 , the calibration work makes the accuracy of the simulated thermal map worse. Hence, we conclude that reducing the magnitude variance can improve the local calibration but has no significant help for global calibration. If enough sensor observations are available we can set a small magnitude variance unless a large one is required. 


\subsubsection{The influence of distance variance}

To check the influence of distance variance, we set $\sigma_{d}$ as $1,0.5,0.2$, and 0.1 , respectively. With the four sensor observations (s1-s4), we estimated the error of the thermal map in Figure 2.7(A). The estimated errors are given in Figure2.12. The error of the estimated thermal map at the 8 sensing locations are shown in Figure 2.13, and the RMSE of the calibrated thermal map at the 8 sensing locations are given in Table 2.4 .

TABLE 2.4: The RMSE of the thermal maps data at the sensing locations w.r.t. different distance variance $\sigma_{d}$

\begin{tabular}{l|cc|cc}
\hline \hline Data at the sensing locations & $\begin{array}{c}\text { RMSE } \\
(\mathrm{s} 1-\mathrm{s} 8)\end{array}$ & $\begin{array}{c}\text { improvement } \\
(\mathrm{s} 1-\mathrm{s} 8)\end{array}$ & $\begin{array}{c}\text { RMSE } \\
(\mathrm{s} 5-\mathrm{s} 8)\end{array}$ & $\begin{array}{c}\text { improvement } \\
(\mathrm{s} 5-\mathrm{s} 8)\end{array}$ \\
\hline CFD simulation result & 1.1197 & $/$ & 1.3518 & $/$ \\
calibrated result $\left(\sigma_{d}=1\right)$ & 0.9558 & $14.64 \%$ & 1.3518 & $0 \%$ \\
calibrated result $\left(\sigma_{d}=0.5\right)$ & 0.9594 & $14.32 \%$ & 1.3568 & $-0.37 \%$ \\
calibrated result $\left(\sigma_{d}=0.2\right)$ & 0.7117 & $36.44 \%$ & 1.0062 & $25.45 \%$ \\
calibrated result $\left(\sigma_{d}=0.1\right)$ & 0.6794 & $39.32 \%$ & 0.8985 & $33.38 \%$ \\
\hline \hline
\end{tabular}

Note: Both $\alpha$ and $\sigma_{m}$ are fixed. We set $\alpha=0.01$ and $\sigma_{m}=1000$. Here, improvement $=($ RMSE of CFD simulation result - RMSE of calibrated result $) /$ RMSE of CFD simulation result.

From Figure 2.12 we can find that like the magnitude variance, the distance variance has no significant influence on the maximum value of the estimated errors. It is clear shown in Figure 2.12 that with a smaller distance variance, the scope of non-zero region of the estimated errors dramatically decrease. Figure 2.13 shows that when the distance variance $\sigma_{d} \leq 0.5$, the proposed method can provide almost perfect calibration at the four sensing locations (s1-s4). Table 2.4 shows that with a smaller distance variance, the errors of the calibrated thermal map at the sensing locations s5-s8 increase. These agree with our previous analysis that the distance variance controls the scope of the region on which the sensor observation has significant influence. A small distance variance $\sigma_{d}$ can lead to very good local calibration while a large $\sigma_{d}$ can provide global influence for an isolated sensor observation. 


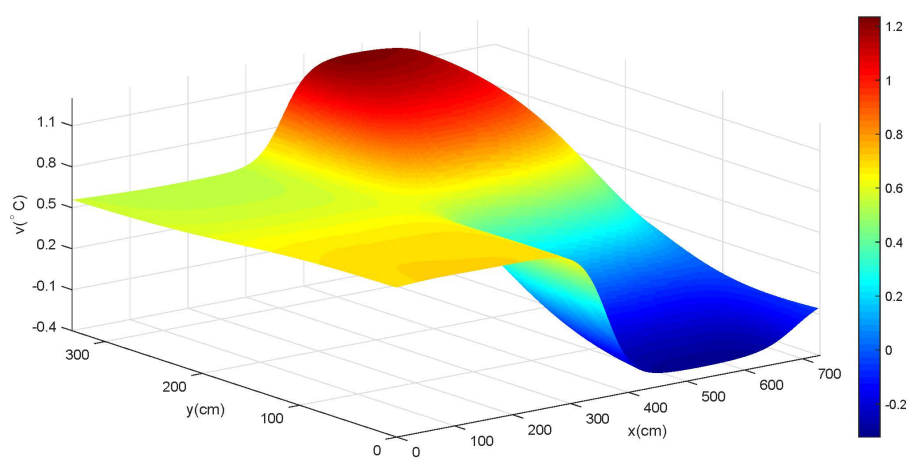

(A) $\sigma_{d}=1$

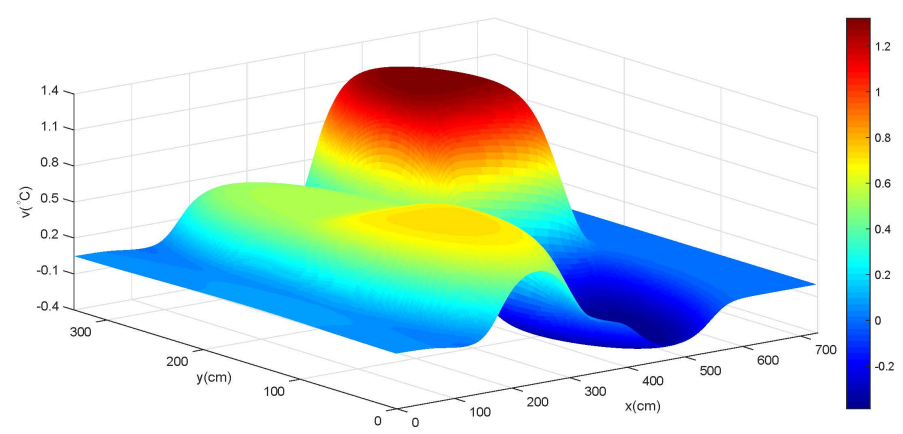

(в) $\sigma_{d}=0.5$

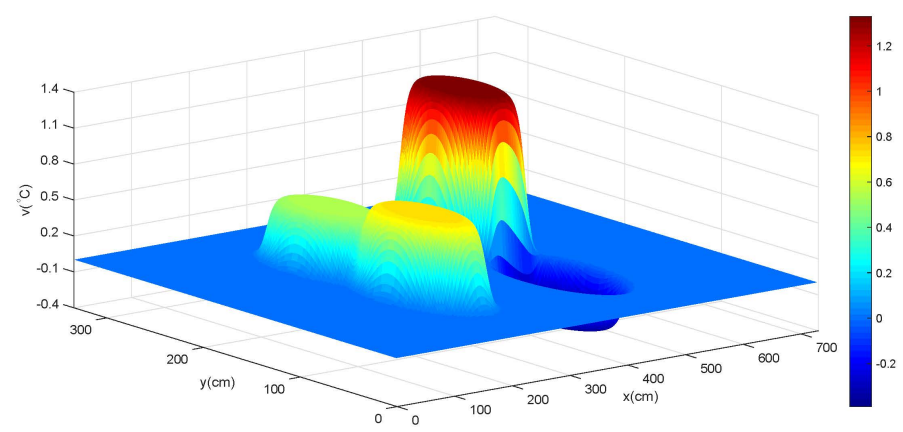

(C) $\sigma_{d}=0.2$

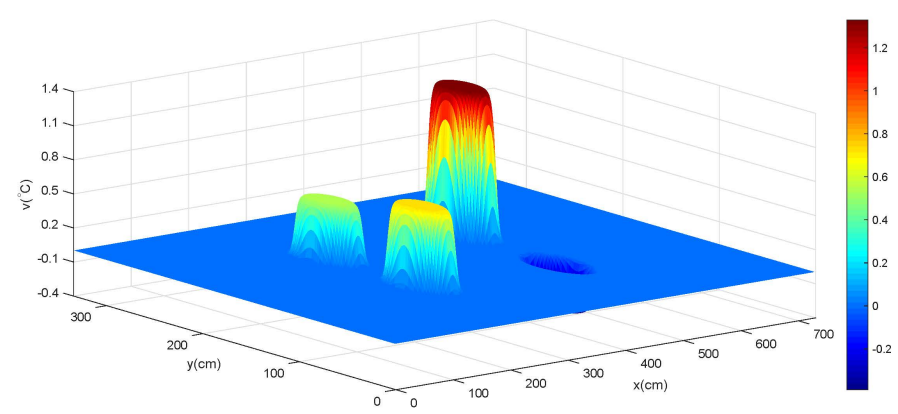

(D) $\sigma_{d}=0.1$

Figure 2.12: The estimated error $(\hat{\mathbf{v}})$ of the thermal map in Figure 2.7(A) w.r.t. different distance variance $\sigma_{d} . \alpha=0.01$ and $\sigma_{m}=1000$. 


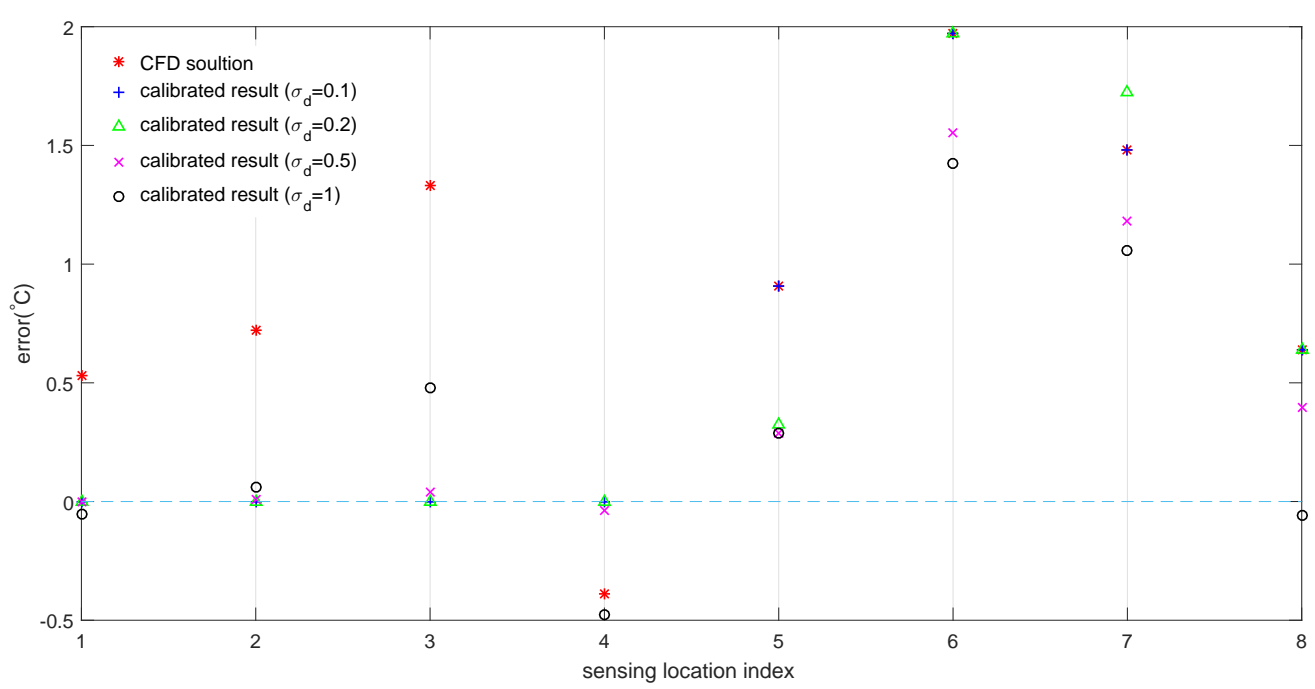

Figure 2.13: The errors of CFD simulation result and the calibrated thermal maps at the sensing locations s1-s8 with $\alpha=0.01$ and $\sigma_{m}=1000$

\subsection{Concluding remarks}

Calibration CFD results with sparse sensor observations is a new and interesting problem. The proposed method can provide an effective solution to this problem. The experimental results show that with four sensor observations (s1-s4), the proposed method has $33.38 \%$ improvement on the accuracy of the simulation data around sensors s5-s8. If we consider the whole thermal map (including the region around sensors s1-s4), the improvement can be even larger.

In the proposed approach, we need to set three parameters for the proposed method: balance factor, magnitude variance, and distance variance. The balance factor controls the magnitude of the adjustment for the simulated thermal map, but the upper bound of this magnitude is determined by the sensing locations. The distance variance controls the scope of the regions on which an isolated sensor observation has significant influence. A small distance variance can lead to very good local calibration while a large can provide global influence for an isolated sensor observation. The magnitude variance controls the influence of the CFD results and scales the influence of the distance variance. Reducing the magnitude variance can improve the local calibration but has no significant help for global 
calibration. If enough sensor observations are available, we can set both small magnitude variance and small distance variance, unless relative larger values of the two variances are proper.

The capacity and the performance of the proposed method is closely related to the number of sensor observations and the sensing locations. The issue of how many sensors are required and where to place them are very interesting topics for our future research. In addition, it is possible for us to design the physical distance and locations between sensors based on the given calibration performance in the future. 



\section{Chapter 3}

\section{Physical field reconstruction}

In Chapter 2, we provided a method to calibrate one simulated physical field with sparse sensor observations. With the proposed method, we can narrow the gap between the CFD simulation results and the experimental data. However, the proposed method is with respect to specific settings, and it can hardly be generalised to improve the simulation results with different input parameters (e.g., parametric boundary conditions). If the input parameters change, both the CFD simulation and the calibration process are required to be repeated. In this chapter, we present a two-stage physical field reconstruction (TSPFR) approach, with which we can directly estimate a physical field of interest from sparse sensor observations without doing a CFD simulation.

This chapter is organized as follows: Section 3.1 describes the physical field reconstruction problem. Section 3.2 shows the procedure of finding the PCA modes and the two current approaches of physical field reconstruction via PCA. In section 3.3, we propose the TSPFR method. In Section 3.4, we compare the error of the new approach with those of current approaches. We discuss the sensor placement in Section 3.5. In Section 3.6, we provide a method for physical field reconstruction when the number of sensor observations is reduced. In Section 3.7, we verify the proposed method for the indoor thermal map estimation problem used in Chapter 2 , and the conclusions are given in Section 3.8. 


\subsection{Problem Formulation}

The physical field data (e.g., temperature, pressure, velocity, etc.) obtained from a CFD simulation can be represented by $s(x, \mathbf{p})$ where $x \in \mathbb{X}=\left\{x_{1}, x_{2}, \ldots, x_{N}\right\}, \mathbb{X} \subset$ $\mathbb{R}^{d}$ is the meshed spatial domain in $d$-dimensional Euclidean space, $d \in\{1,2,3\}, N$ is the number of points of the mesh grid which is generally from tens of thousands to millions, $\mathbf{p} \in \mathbb{P} \subset \mathbb{R}^{p}$ is a vector which consists of all the varying input parameters using in the CFD simulations, and $\mathbb{P}$ is the set of the parameters within the range of interest.

The input parameters of a CFD simulation includes a series of parameters. In many applications, most of the input parameters are fixed but a part of them are varying, e.g., Rayleigh number [53, 56], Mach number [54, 55], and boundary condition parameters $[55,56,84,85]$. These varying parameters often have a great influence on the simulation results. In many applications, the varying input parameters are often observed, and we denote it by

$$
\mathbf{p}_{\mathrm{o}}=\mathbf{p}+\mathbf{v}_{\mathrm{p}}
$$

where $\mathbf{v}_{\mathrm{p}}$ is the corresponding measurement noise.

With the observed input parameters, the physical field of interest can be obtained from CFD simulation. If the input parameters change, so does the physical field. Therefore, the simulation needs to be rerun. To save the computational cost of the endless simulation, an alternative approach that can quickly reconstruct the physical field is highly desirable.

In practice, some sparse observations of one physical field can be obtained from a sensor network, which can be described as

$$
y(x)=s(x, \mathbf{p})+v(x), \quad x \in \mathbb{X}_{\mathrm{s}}=\left\{x_{l_{1}}, x_{l_{2}}, \ldots, x_{l_{m}}\right\}, \mathbf{p} \in \mathbb{P}
$$


where $v(x)$ is the measurement noise, $\mathbb{X}_{\mathrm{s}} \subset \mathbb{X}, m \ll N$ is the sensor number, and $x_{l_{i}}$ represents the $i$-th sensing location. We assume that the sensor noise $v\left(x_{l_{1}}\right), v\left(x_{l_{2}}\right), \ldots, v\left(x_{l_{m}}\right)$ are independent identically distributed $\mathcal{N}\left(0, \sigma^{2}\right)$ random variables.

For simplicity, we can array the physical field data into a more compact vector form $\mathbf{s}(\mathbf{p})$. Note that $\mathbf{s}(\mathbf{p}) \in \mathbb{R}^{N}$ with components $s_{i}=s\left(x_{i}, \mathbf{p}\right)$. Similarly, we array all the sensor observations as a column vector and formulate it as the following measurement model

$$
\mathbf{y}=\mathbf{H s}(\mathbf{p})+\mathbf{v}
$$

where $\mathbf{y}=\left[y\left(x_{l_{1}}\right), y\left(x_{l_{2}}\right), \cdots, y\left(x_{l_{m}}\right)\right]^{\mathrm{T}}, \mathbf{v}=\left[v\left(x_{l_{1}}\right), v\left(x_{l_{2}}\right), \cdots, v\left(x_{l_{m}}\right)\right]^{\mathrm{T}}$, and $\mathbf{H} \in$ $\mathbf{R}^{m \times N}$ is the sampling kernel whose $i$-th row is the same as the $l_{i}$-th row of the identify matrix $\mathbf{I}_{N \times N}$ for all $1 \leq i \leq m$.

It is assumed that one database obtained from a series of CFD simulations, $\mathbf{S}=$ $\left[\mathbf{s}\left(\mathbf{p}_{1}\right), \mathbf{s}\left(\mathbf{p}_{2}\right), \ldots, \mathbf{s}\left(\mathbf{p}_{M}\right)\right]$ associated with the sampled parameters $\mathbb{P}_{\mathbf{s}}=\left\{\mathbf{p}_{1}, \mathbf{p}_{2}, \ldots, \mathbf{p}_{M}\right\} \subseteq$ $\mathbb{P}$, is available. To build the database (i.e., the matrix $\mathbf{S}$ ), the CFD model should be well calibrated. We can further enhance the simulation results using the method provided in Chapter 2 to guarantee a good match between the simulated physical fields and the real physical fields. Then, we formulate the following physical field reconstruction problem.

Problem 1. Given one physical field database $\mathbf{S}$, how can we quickly reconstruct the physical field $\mathbf{s}(\mathbf{p})$ from the sparse observations $\mathbf{y}$ and the observed parameters $\mathbf{p}_{\mathrm{o}}$ ?

In this chapter, we focus on this problem and try to reconstruct the physical field of interest with as small a number of sensor observations as we can. Without loss of generality, in the sequel we use a lowercase (e.g., s) to represent the physical field data at a particular position or one element of a vector, and a bold letter (e.g., s) to represent a vector or a physical field. 
This is an inverse problem. Principal components analysis (PCA) is the most popular tool to solve this inverse problem. Next, we give a brief review on the PCA and the state-of-the-art of of physical field reconstruction via PCA.

\subsection{PCA for physical field reconstruction}

\subsubsection{PCA modes of the physical fields}

For a physical field, the deviation from the mean is given by

$$
\mathbf{w}(\mathbf{p})=\mathbf{s}(\mathbf{p})-\overline{\mathbf{s}}
$$

where

$$
\overline{\mathbf{s}}=\frac{\int_{\mathbb{P}} \mathbf{s}(\mathbf{p}) \mathrm{d} \mathbf{p}}{\int_{\mathbb{P}} \mathrm{d} \mathbf{p}}
$$

is the mean of the physical field $\mathbf{s}(\mathbf{p})$ in terms of the input parameters $\mathbf{p}$.

The PCA modes of the physical field is a set of orthonormal vectors $\left\{\boldsymbol{\varphi}_{k} \mid k \in \mathbb{I}, \boldsymbol{\varphi}_{k} \in\right.$ $\left.\mathbb{R}^{N}\right\}$ with index set $\mathbb{I}=\{1,2, \ldots, N\}$ such that, for any $\mathbf{p} \in \mathbb{P}$ and $n \in \mathbb{I}$,

$$
\mathbf{w}(\mathbf{p})=\sum_{k=1}^{N} c_{k}(\mathbf{p}) \boldsymbol{\varphi}_{k}=\sum_{k=1}^{N}\left(\mathbf{w}(\mathbf{p}), \boldsymbol{\varphi}_{k}\right) \boldsymbol{\varphi}_{k}=\sum_{k=1}^{n}\left(\mathbf{w}(\mathbf{p}), \boldsymbol{\varphi}_{k}\right) \boldsymbol{\varphi}_{k}+\boldsymbol{\epsilon}_{n}(\mathbf{p})
$$

and the energy of truncation error $\boldsymbol{\epsilon}_{n}$

$$
\Xi_{n}=\int_{\mathbb{P}}\left\|\mathbf{w}(\mathbf{p})-\sum_{k=1}^{n}\left(\mathbf{w}(\mathbf{p}), \boldsymbol{\varphi}_{k}\right) \boldsymbol{\varphi}_{k}\right\|_{2}^{2} \mathrm{~d} \mathbf{p}
$$

is minimized, where $(\cdot, \cdot)$ is the inner product operator, and

$$
c_{k}(\mathbf{p})=\left(\mathbf{w}(\mathbf{p}), \varphi_{k}\right)
$$

Directly minimizing $\Xi_{n}$ is not easy. One popular approach to find the approximated solution is the method of snapshots [57-60], which only consider the physical field 
(i.e., the snapsots) in the known database $\mathbf{S}$. In other words, we can approximately set

$$
\overline{\mathbf{s}}=\frac{1}{M} \sum_{i=1}^{M} \mathbf{s}\left(\mathbf{p}_{i}\right)
$$

and find the PCA modes, i.e., the dominant orthonormal basis of the spanned space $V=\operatorname{span}\left\{\mathbf{w}\left(\mathbf{p}_{1}\right), \mathbf{w}\left(\mathbf{p}_{2}\right), \cdots, \mathbf{w}\left(\mathbf{p}_{M}\right)\right\}$, by solving

$$
\min \sum_{i=1}^{M}\left\|\mathbf{w}\left(\mathbf{p}_{i}\right)-\sum_{k=1}^{n}\left(\mathbf{w}\left(\mathbf{p}_{i}\right), \boldsymbol{\varphi}_{k}\right) \boldsymbol{\varphi}_{k}\right\|_{2}^{2}
$$

The solution of the minimization problem (3.4) can be obtained from the following eigenvalue problem [51]

$$
\mathbf{W} \mathbf{W}^{\mathrm{T}} \boldsymbol{\varphi}_{k}=\mu_{k} \boldsymbol{\varphi}_{k}, \quad k \in \mathbb{I}
$$

where $\mathbf{W}=\left[\mathbf{w}\left(\mathbf{p}_{1}\right), \mathbf{w}\left(\mathbf{p}_{2}\right), \ldots, \mathbf{w}\left(\mathbf{p}_{M}\right)\right], \mu_{k} \geq \mu_{l} \geq 0$ and $\left\|\boldsymbol{\varphi}_{k}\right\|_{2}=1$ for all $1 \leq k \leq l \leq M$, and $\mu_{k} \equiv 0$ for all $k>M$ due to the rank of $\mathbf{W W}^{\mathrm{T}}, r \leq M$.

Generally $M \ll N$, to save computational cost, we can obtain the first $M$ PCA modes from

$$
\boldsymbol{\varphi}_{k}=\operatorname{Norm}\left(\mathbf{W} \phi_{k}\right), \quad k=1,2, \ldots, M
$$

where $\operatorname{Norm}(\cdot)$ is the normalization operator, and $\phi_{k}$ satisfies

$$
\mathbf{W}^{\mathrm{T}} \mathbf{W} \phi_{k}=\mu_{k} \boldsymbol{\phi}_{k}, \quad k=1,2, \ldots, M
$$

with $\mathbf{W}^{\mathrm{T}} \mathbf{W} \in \mathbb{R}^{M \times M}$. Since $M \ll N$, solving the eigenvalue problem (3.7) is computationally more efficient than (3.5). The other PCA modes (i.e., $\boldsymbol{\varphi}_{k}, M<$ $k \leq N)$ are the orthonormal basis of $V^{\perp}$, which is the orthogonal complementary space of $V$. In practice, if the number of snapshots is large enough, we do not need to compute the non-dominant PCA modes, $\varphi_{k}, M<k \leq N$, because they are insignificant in the recovery of the physical fields . 
With the PCA modes $\left\{\boldsymbol{\varphi}_{k}, k \in \mathbb{I}\right\}$, we can obtain the following spectral expansion of any physical field, $\mathbf{s}(\mathbf{p}), \mathbf{p} \in \mathbb{P}$

$$
\begin{aligned}
\mathbf{s}(\mathbf{p}) & =\overline{\mathbf{s}}+\sum_{k=1}^{n} c_{k}(\mathbf{p}) \boldsymbol{\varphi}_{k}+\sum_{k=n+1}^{N} c_{k}(\mathbf{p}) \boldsymbol{\varphi}_{k} \\
& =\overline{\mathbf{s}}+\tilde{\boldsymbol{\Phi}} \mathbf{c}+\tilde{\boldsymbol{\Phi}}_{\text {tail }} \mathbf{c}_{\text {tail }}
\end{aligned}
$$

where $n$ is the truncation level, $c_{k}(\mathbf{p})=c_{k}=\left(\mathbf{w}(\mathbf{p}), \boldsymbol{\varphi}_{k}\right)$ is the $k$-th PCA coefficient, $\mathbf{c}=\left[c_{1}, c_{2}, \ldots, c_{n}\right]^{\mathrm{T}}, \mathbf{c}_{\text {tail }}=\left[c_{n+1}, c_{n+2}, \ldots, c_{N}\right]^{\mathrm{T}}, \tilde{\boldsymbol{\Phi}}=\left[\boldsymbol{\varphi}_{1}, \boldsymbol{\varphi}_{2}, \ldots, \boldsymbol{\varphi}_{n}\right] \in \mathbb{R}^{N \times n}$, and $\tilde{\boldsymbol{\Phi}}_{\text {tail }}=\left[\boldsymbol{\varphi}_{n+1}, \boldsymbol{\varphi}_{n+2}, \ldots, \boldsymbol{\varphi}_{N}\right]$.

Then, the truncation error can be rewritten as

$$
\boldsymbol{\epsilon}_{n}(\mathbf{p})=\tilde{\boldsymbol{\Phi}}_{\text {tail }} \mathbf{c}_{\text {tail }}
$$

For any physical field $\mathbf{s}(\mathbf{p}), \mathbf{p} \in \mathbb{P}$, if the truncation error is small enough, $\mathbf{s}(\mathbf{p})$ can be approximated by a combination of the dominant PCA modes, that is,

$$
\mathbf{s}(\mathbf{p}) \approx \overline{\mathbf{s}}+\sum_{k=1}^{n} c_{k} \boldsymbol{\varphi}_{k}=\overline{\mathbf{s}}+\tilde{\mathbf{\Phi}} \mathbf{c}
$$

where $n \leq M$ is the truncation level. According to the definition of PCA modes, we cannot find a better approximation with only $n$ orthonormal basis vectors. The truncation level should guarantee that, for any physical field, $\mathbf{s}(\mathbf{p}), \mathbf{p} \in \mathbb{P}$, the energy lost by the approximated representation (3.9) is insignificant. We hope that the truncation level $n$ is small and hence we can reconstruct the physical field using a small number of PCA modes. To find the smallest but acceptable truncation level $n$, theoretically we should check all the physical fields $\mathbf{s}(\mathbf{p}), \mathbf{p} \in \mathbb{P}$, which, in practice, are replaced by the ensemble snapshots $\left\{\mathbf{s}\left(\mathbf{p}_{i}\right)\right\}_{i=1}^{M}$. The energy of truncation error of the ensemble snapshots satisfies [50]

$$
\sum_{i=1}^{M}\left\|\boldsymbol{\epsilon}_{n}\left(\mathbf{b}_{i}\right)\right\|^{2}=\sum_{k=n+1}^{N} \mu_{k}=\sum_{k=n+1}^{M} \mu_{k}
$$


The eigenvalue $\mu_{k}$ represents the relative importance of the PCA mode $\boldsymbol{\varphi}_{k}[59,68]$, and the truncation level $n$ can be determined by the criterion

$$
P_{n}=\frac{\sum_{i=1}^{M}\left\|\mathbf{w}\left(\mathbf{p}_{i}\right)-\boldsymbol{\epsilon}_{n}\left(\mathbf{p}_{i}\right)\right\|^{2}}{\sum_{i=1}^{M}\left\|\mathbf{w}\left(\mathbf{p}_{i}\right)\right\|^{2}}=\frac{\sum_{k=1}^{n} \mu_{k}}{\sum_{k=1}^{M} \mu_{k}} \geq P_{\mathrm{tol}}
$$

If the correlation tolerance $P_{\text {tol }}$ is high enough, e.g., over $99 \%$ [68], then the first $n$ PCA modes are adequate to capture the principal features and to approximately reconstruct the physical field of interest.

Theoretically, a CFD simulation cannot provide the exact physical field; therefore, the PCA modes obtained from the proposed procedure are not the PCA modes of the real physical fields but those of the CFD results. Nevertheless, in practice, we use them as the approximated PCA modes of the real physical fields; hence, both the CFD model and the simulated physical fields should be well calibrated to guarantee the reliability of the PCA modes. In the sequel of this chapter, we assume that all the CFD results are reliable and we do not distinguish the simulated physical fields and the real fields, unless a specific statement is given.

In (3.9), $\overline{\mathbf{s}}$ and $\tilde{\boldsymbol{\Phi}}$ are known, and once we can estimate the dominant PCA coefficients c, the physical field can be approximately reconstructed. Currently the following two approaches are commonly used to estimate the PCA coefficients.

\subsubsection{PCA coefficients estimation from the observed input parameters}

This method has been discussed in [53-55]. Based on the database $\mathbf{S}$, the regression models between each PCA coefficient $c_{k}, k \in \mathcal{I}_{\overline{0}}=\{1,2, \ldots, \check{n}\}$ and the input parameters $\mathbf{p}$ can be constructed. To estimate these regression models, the spline interpolation technique is commonly used [53-55], which find the regression model 
by minimizing [52]:

$$
E\left[f_{k}\right]=\sum_{i=1}^{M}\left|f_{k}\left(\mathbf{p}_{i}\right)-c_{k}\left(\mathbf{p}_{i}\right)\right|^{2}+\lambda \int_{\mathbb{P}}\left|D^{2} f_{k}\right|^{2} d \mathbf{p}, k \in \mathcal{I}_{\overline{0}}
$$

where $f_{k}(\cdot)$ is the regression function between $c_{k}$ and the parameter $\mathbf{p}, \lambda>0$ is a priori chosen smoothing parameter, $D^{2} f_{k}$ is the matrix of second-order partial derivatives of $f_{k}$, and $\left|D^{2} f_{k}\right|^{2}$ is the sum of squares of the matrix entries.

Here, $\check{n}(n \leq \check{n} \leq M)$ is the number of involved PCA modes. For any $k>\check{n}$ and $\mathbf{p} \in \mathbb{P}_{\mathrm{s}}$, the PCA coefficient satisfies

$$
\left|c_{k}\right|=\left|\left(\boldsymbol{\varphi}_{k}, \mathbf{w}(\mathbf{p})\right)\right|<\varepsilon
$$

where $\varepsilon$ is a small positive number.

For more details on training the regression model, do refer to [52]. The minimizer of (3.11) is

$$
f_{k}(\mathbf{p})=\sum_{i=1}^{M} a_{k i} G\left(\mathbf{p}, \mathbf{p}_{i}\right)+\left[\begin{array}{ll}
\mathbf{p}^{\mathrm{T}} & 1
\end{array}\right] \mathbf{b}_{k}, \quad k \in \mathcal{I}_{\overline{0}}
$$

where $a_{k i}, 1 \leq i \leq M$ and $\mathbf{b}_{k} \in \mathbb{R}^{p+1}$ are the parameters to be determined. $G\left(\mathbf{p}, \mathbf{p}_{i}\right)$ is the Green's function of the biharmonic equation

$$
\Delta^{2} G\left(\mathbf{x}, \mathbf{p}_{i}\right)=\delta\left(\left\|\mathbf{x}-\mathbf{p}_{i}\right\|_{2}\right)
$$

where $\Delta$ is the Laplacian operator, and $\delta(\cdot)$ is the Dirac delta function.

For simplicity, we denote $\mathbf{a}_{k}=\left[a_{k 1}, a_{k 2}, \cdots, a_{k M}\right]^{\mathrm{T}}$. With the known PCA coefficients of the physical fields in $\mathbf{S}$, we can find the parameters $\mathbf{a}_{k}$ and $\mathbf{b}_{k}$. From the constraints

$$
c_{k}^{(i)}=f_{k}\left(\mathbf{p}_{i}\right) \quad k \in \mathcal{I}_{\overline{0}}, 1 \leq i \leq M
$$

We can obtain

$$
\mathbf{c}_{k}=A \mathbf{a}_{k}+B \mathbf{b}_{k}, \quad k \in \mathcal{I}_{\overline{0}}
$$


where $\mathbf{c}_{k}=\left[c_{k}^{(1)}, c_{k}^{(2)}, \cdots, c_{k}^{(M)}\right]^{\mathrm{T}}, A_{i j}=G\left(\mathbf{p}_{i}, \mathbf{p}_{j}\right)$, and $B \in \mathbb{R}^{M \times(p+1)}$ whose $i$-th row is $\left[\begin{array}{ll}\mathbf{p}_{i}^{T} & 1\end{array}\right]$. Considering the orthogonality condition, which comes from the functional analysis in [86], $B^{\mathrm{T}} \mathbf{a}_{k}=\mathbf{0}$, we can obtain the solution

$$
\begin{aligned}
\mathbf{a}_{k} & =A^{-1}\left(\mathbf{c}_{k}-B \mathbf{b}_{k}\right) \\
\mathbf{b}_{k} & =\left(B^{\mathrm{T}} A^{-1} B\right)^{-1} B^{\mathrm{T}} A^{-1} \mathbf{c}_{k}
\end{aligned}
$$

With $\mathbf{a}_{k}$ and $\mathbf{b}_{k}$, we can obtain the minimizer $f_{k}$ for all $k \in \mathcal{I}_{\overline{0}}$. With the regression functions $f_{k}, k \in \mathcal{I}_{\overline{0}}$, we can obtain an estimate of the physical field:

$$
\check{\mathbf{s}}(\mathbf{p})=\overline{\mathbf{s}}+\check{\mathbf{w}}(\mathbf{p})=\overline{\mathbf{s}}+\check{\mathbf{\Phi}} \check{\mathbf{c}}(\mathbf{p})
$$

where $\check{\mathbf{\Phi}}=\left[\varphi_{1}, \varphi_{2}, \ldots, \varphi_{\check{n}}\right], \check{\mathbf{c}}(\mathbf{p})=\left[\check{c}_{1}, \check{c}_{2}, \ldots, \check{c}_{\check{n}}\right]^{\mathrm{T}}$, and

$$
\check{c}_{k}=f_{k}\left(\mathbf{p}_{\mathrm{o}}\right), \quad k \in \mathcal{I}_{\overline{0}}
$$

where $\mathbf{p}_{\mathrm{o}}$ consists of the observed input parameters.

In (3.13), the Green's function is

$$
G\left(\mathbf{p}, \mathbf{p}_{i}\right)= \begin{cases}\alpha_{1}\left\|\mathbf{p}-\mathbf{p}_{i}\right\|_{2}^{4-p} \ln \left(\left\|\mathbf{p}-\mathbf{p}_{i}\right\|_{2}\right) & p=2 \text { or } p=4 \\ \alpha_{2}\left\|\mathbf{p}-\mathbf{p}_{i}\right\|_{2}^{4-p} & \text { others }\end{cases}
$$

Here $p$ is the number of the input parameters of interest used in CFD simulations. If $p \geq 4$, Green's function $G\left(\mathbf{p}, \mathbf{p}_{i}\right)$ has singularity at $\mathbf{p}_{i}$. In practice, if $p \geq 4$, we usually use $G\left(\mathbf{p}, \mathbf{p}_{i}\right)=\left\|\mathbf{p}-\mathbf{p}_{i}\right\|_{2}^{2}$ instead, but then $G\left(\mathbf{p}, \mathbf{p}_{i}\right)$ is no longer the solution of (3.14) and $f_{k}$ is not the minimizer of (3.11).

Remark 1. If $p$, the number of the input parameters, is not less than 4 , the performance of spline interpolation will be discounted because of the approximated Green's functions.

Remark 2. If we estimate the indoor physical field from the input parameters, all the involved PCA coefficients can be estimated ( $\check{n}$ is probably larger than $n$ ), which leads to a very small truncation error. 
Remark 3. The spline interpolation is used to train the multi-input-single-output regression models. From each regression model we can only obtain one PCA coefficient. Therefore, to find all involved PCA coefficients, we need to train $\check{n}$ regression models. Equation (3.12) indicate that $\check{n}$ is a number larger than the number of dominant PCA modes i.e., $n$.

\subsubsection{Dominant PCA coefficients estimation from sparse observations}

Here, we review a method to estimate the dominant PCA coefficients from sparse observations of the physical field. This method has been discussed in [57-59].

Substituting (3.9) into (3.1) yields

$$
\mathbf{y} \approx \mathbf{H} \overline{\mathbf{s}}+\mathbf{H} \tilde{\Phi} \mathbf{c}+\mathbf{v}=\mathbf{H} \overline{\mathbf{s}}+\mathbf{\Phi} \mathbf{c}+\mathbf{v}
$$

where $\boldsymbol{\Phi}=\mathbf{H} \tilde{\boldsymbol{\Phi}} \in \mathbb{R}^{m \times n}$ is a pruned matrix from some rows of $\tilde{\boldsymbol{\Phi}}$ indexed by $\mathcal{L}=\left\{l_{1}, l_{2}, \ldots, l_{m}\right\}$. Then, using the least square technique, we can easily obtain the dominant PCA coefficients from (3.16), i.e.,

$$
\hat{\mathbf{c}}=\boldsymbol{\Phi}^{\dagger}(\mathbf{y}-\mathbf{H} \overline{\mathbf{s}})=\left(\boldsymbol{\Phi}^{\mathrm{T}} \boldsymbol{\Phi}\right)^{-1} \boldsymbol{\Phi}^{\mathrm{T}}(\mathbf{y}-\mathbf{H} \overline{\mathbf{s}})
$$

where $\boldsymbol{\Phi}^{\dagger}$ is the Moore-Penrose inverse of matrix $\boldsymbol{\Phi}$. Here, the non-singularity of $\boldsymbol{\Phi}^{\mathrm{T}} \boldsymbol{\Phi}$ implies that the number of sensor observations $m$ cannot be less than the truncation level $n$.

Then, the physical field can be reconstructed by

$$
\hat{\mathbf{s}}_{n}=\overline{\mathbf{s}}+\hat{\mathbf{w}}_{n}=\overline{\mathbf{s}}+\tilde{\boldsymbol{\Phi}} \hat{\mathbf{c}}=\overline{\mathbf{s}}+\tilde{\boldsymbol{\Phi}} \Phi^{\dagger}(\mathbf{y}-\mathbf{H} \overline{\mathbf{s}})
$$

where $\hat{\mathbf{s}}_{n}$ and $\hat{\mathbf{w}}_{n}$ are the reconstructed physical field and its deviation from the mean $\overline{\mathbf{s}}$, respectively. The subscript $n$ in (3.18) represents the truncation level, i.e., the number of used dominant PCA modes. 
For clarity, we use $\hat{\mathbf{c}}$ to represent the estimated dominant PCA coefficients from sparse sensor observations, and $\hat{\mathbf{c}}=\left[\hat{c}_{1}, \hat{c}_{2}, \cdots, \hat{c}_{n}\right]$. For all the involved PCA coefficients estimated from a regression model with the observed input parameters, we represent them by $\check{\mathbf{c}}$, and $\check{\mathbf{c}}=\left[\check{c}_{1}, \check{c}_{2}, \cdots, \check{c}_{\check{n}}\right]$.

Remark 4. In this method, only the dominant PCA components are considered. If the observations are accurate enough and the sensing locations are well selected, this method outperforms the regression model in the sense of estimating the dominant PCA coefficients. However, it is often expensive to estimate all the $\check{n}$ involved PCA coefficients, which requires at least $\check{n}$ sensor observations.

\subsection{Two-stage physical field reconstruction}

Remarks 1-4 highlight the merits and demerits of the current two physical field reconstruction approaches. In this section, we will detail a new approach, named two-stage physical field reconstruction (TSPFR) to this problem. The new approach can fuse the two current approaches to achieve better performance. The block diagram of TSPFR is shown in Figure 1.4. For clarity, we re-show it here (see Figure 3.1).

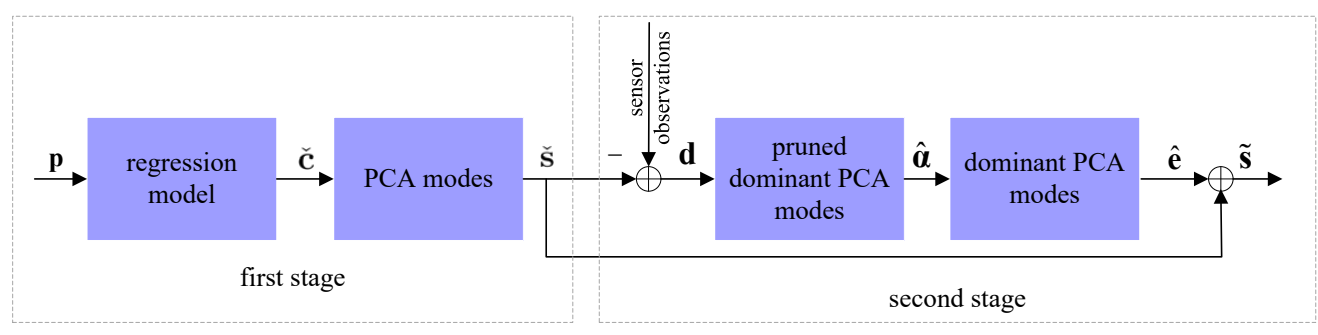

FigURE 3.1: The block diagram of the two-stage physical field reconstruction 


\subsubsection{First-stage: physical field reconstruction from ob- served input parameters}

In the first stage, we estimate all the involved PCA coefficients $\check{c}_{k}, k \in \mathcal{I}_{\overline{0}}$ from the observed input parameters. Like the current method in section 3.2.2, we need to build a regression model between the PCA coefficients and the input parameters. Here, we use the scaled extreme learning machine (sELM) instead of the spline interpolation. Firstly, we show how the standard extreme learning machine (ELM) is applied for physical field reconstruction, and then we modify the standard ELM as the sELM to achieve better estimation performance.

\subsubsection{Standard ELM for physical field reconstruction}

The standard ELM is a fast way to train the single-hidden-layer feedforward neuralnetworks (SLFNs). When using ELM, the input-to-hidden layer connection weights are randomly generated without tuning and are independent of the training data. Therefore, ELM is computationally much more efficient than many other machine learning techniques in the training process. This idea has been discussed in [8790], and ELM was first coined in [91]. For more details about ELM, do refer to [61, 91-93].

A SLFN, as shown in Figure 3.2, can act as a nonlinear regression model between the input data (input parameters) and output data (PCA coefficients). The output of the neural network can be written as:

$$
\mathbf{c}(\mathbf{p})=\sum_{i=1}^{L} \boldsymbol{\beta}_{i} g_{i}(\mathbf{p})=\sum_{i=1}^{L} \boldsymbol{\beta}_{i} g\left(\mathbf{r}_{i}^{\mathrm{T}} \mathbf{p}+\varepsilon_{i}\right)
$$

where $\mathbf{c}(\mathbf{p})=\left[c_{1}, c_{2}, \cdots, c_{\check{n}}\right]^{\mathrm{T}}$ represents the involved PCA coefficients of $\mathbf{s}(\mathbf{p})$. $\boldsymbol{\beta}=\left[\boldsymbol{\beta}_{1}, \boldsymbol{\beta}_{2}, \cdots, \boldsymbol{\beta}_{L}\right]^{\mathrm{T}} \in \mathbb{R}^{L \times \check{n}}$ is the output weights from the hidden nodes to the output nodes, $L$ is the number of hidden neuron, $\mathbf{r}_{i} \in \mathbb{R}^{p}$ is the random weights from the input nodes to the $i$-th hidden neuron, $\varepsilon_{i}$ is the random bias of $i$-th 
hidden neuron, and $g(\cdot)$ is a known activation function called ELM random feature mapping [92].

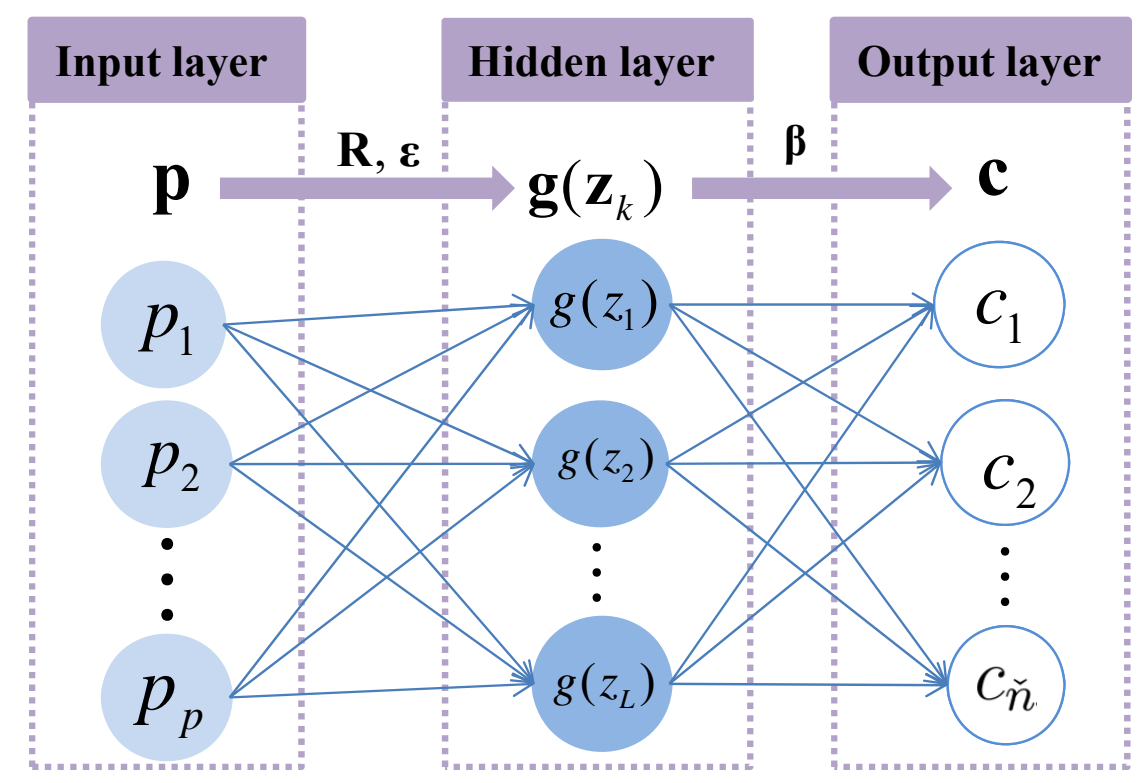

FIgURE 3.2: The structure of the standard extreme learning machine (ELM).

Equation (3.19) can be rewritten as the following compact form:

$$
\mathrm{G} \boldsymbol{\beta}=\mathrm{C}
$$

where $\mathbf{G}$ is the hidden layer output matrix:

$$
\mathbf{G}=\left[\begin{array}{c}
\mathbf{g}\left(\mathbf{p}_{1}\right) \\
\vdots \\
\mathbf{g}\left(\mathbf{p}_{M}\right)
\end{array}\right]=\left[\begin{array}{ccc}
g_{1}\left(\mathbf{p}_{1}\right) & \cdots & g_{L}\left(\mathbf{p}_{1}\right) \\
\vdots & \vdots & \vdots \\
g_{1}\left(\mathbf{p}_{M}\right) & \cdots & g_{L}\left(\mathbf{p}_{M}\right)
\end{array}\right]
$$

and $\mathbf{C}=\left[\mathbf{c}_{1}, \mathbf{c}_{2}, \cdots, \mathbf{c}_{M}\right]^{\mathrm{T}}$. Here

$$
g_{i}\left(\mathbf{p}_{j}\right)=g\left(\mathbf{r}_{i}^{\mathrm{T}} \mathbf{p}_{j}+\varepsilon_{i}\right)
$$


An robust solution of (3.20) is given by [94]

$$
\boldsymbol{\beta}=\left(\gamma \mathbf{I}+\mathbf{G}^{\mathrm{T}} \mathbf{G}\right)^{-1} \mathbf{G}^{\mathrm{T}} \mathbf{C}
$$

where $\gamma$ is the regularization parameter.

Then, the PCA coefficients can be estimated via ELM as

$$
\check{\mathbf{c}}(\mathbf{p})=\mathbf{g}\left(\mathbf{p}_{\mathrm{o}}\right) \boldsymbol{\beta}
$$

With $\check{\mathbf{c}}(\mathbf{p})$, the physical field can be approximated by

$$
\check{\mathbf{s}}(\mathbf{p})=\overline{\mathbf{s}}+\check{\mathbf{w}}(\mathbf{p})=\overline{\mathbf{s}}+\check{\mathbf{\Phi}} \check{\mathbf{c}}(\mathbf{p})
$$

Note that for the results of the first stage, we use $\check{\mathbf{c}}(\mathbf{p}), \check{\mathbf{s}}(\mathbf{p})$, and $\check{\mathbf{w}}(\mathbf{p})$ to respectively represent the estimated PCA coefficients, the reconstructed physical field and its deviation from the mean, no matter how the regression model is trained.

\subsubsection{Scaled ELM for physical field reconstruction}

As shown in Figure 3.2, $z_{i}$ is the input of the activation function. Here

$$
z_{i}=\mathbf{r}_{i}^{\mathrm{T}} \mathbf{p}+\varepsilon_{i}
$$

One example for the activation function is the sigmoid function:

$$
g(z)=\frac{1}{1+\exp (-z)}
$$

which is shown in Figure 3.3. We can find from Figure 3.3 that $g\left(z_{i}\right) \approx 0$ and $g\left(z_{i}\right) \approx 1$ for all $z_{i}<-4$ and $z_{i}>4$, respectively. Therefore, for any $\mathbf{p}$ which can guarantee $\left|z_{i}\right|>4$, the output of the hidden neuron will be 0 or 1 , and the result 
is non-sensitive to the exact value of $\mathbf{p}$. In other words, ELM cannot distinguish the input layer data $\mathbf{p}$ if $\mathbf{p}$ can lead to $\left|z_{i}\right|>4$.

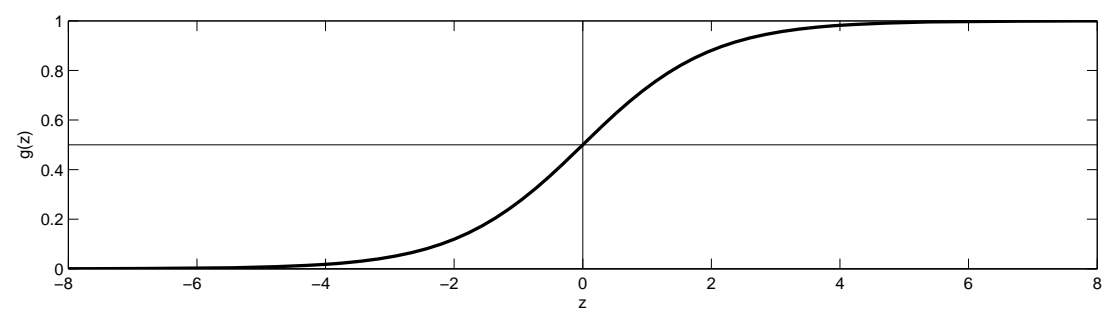

Figure 3.3: The sigmoid function.

When using the standard ELM for physical field reconstruction, we found that most of the hidden neurons of the SLFNs are always 0 or 1 , which leads to a low accuracy estimation. This phenomenon may have insignificant influence on a simple classification problem, but it does lead to poor results for many regression problems. Considering the ELM universal approximation capability theorems [92, 95-97], if we use enough hidden neurons we may still obtain accurate results. However, with more hidden neurons, the computational cost in the training process is higher. All the other ELM random feature mappings mentioned in [92] suffer from similar problems. In this work, we use the sigmoid function as the activation function, and similar steps can be taken if another activation function is used.

In addition, for the physical field reconstruction problem, the input layer data (i.e., p, the input parameters of CFD simulation ) may consist of various types of data, e.g., the cooling air temperature of air-conditioners, the cooling air velocity, the heat flux of heaters, etc. The unit of each type of input parameter is probably different from each other. The magnitude of each type of input parameter may differ significantly from each other. The magnitude of heat flux of electric appliances is typically from several tens to a few hundreds ( $\mathrm{W} / \mathrm{m}^{2}$ i.e., watt-per-square-meter), while the values of cooling air velocity of one air-conditioner is much smaller, definitely less than ten $(\mathrm{m} / \mathrm{s})$. In such a case, the influence of one type input parameter (e.g., heat flux of one heater) with large magnitude overwhelms that of the type 
with small magnitude (e.g., the air velocity), which would adversely affect the training of the regression model.

To overcome the aforementioned two issues, we take the following two steps to modify the standard ELM:

1. Normalize each type input parameter.

2. Scale the random input-to-hidden layer weights.

We call the modified ELM as scaled extreme learning machine (sELM). Next, we detail our proposed sELM.

Firstly, we normalize each type input parameter. We denote the input parameters by

$$
\mathbf{p}=\left[\begin{array}{c}
\mathbf{p}^{(1)} \\
\mathbf{p}^{(2)} \\
\vdots \\
\mathbf{p}^{\left(n_{p}\right)}
\end{array}\right]
$$

where $\mathbf{p}^{(i)}$ represents the $i$-th type input parameter. Before using the input parameters as the input layer data of the standard ELM, we scale the input parameters by

$$
\mathbf{p}_{\mathrm{s}}=S_{\mathrm{p}} \mathbf{p}=\left[\begin{array}{cccc}
\frac{1}{p_{\max }^{(1)}} & & & \\
& \frac{1}{p_{\max }^{(2)}} & & \\
& & \ddots & \\
& & & \frac{1}{p_{\max }^{\left(p_{p}\right)}}
\end{array}\right]\left[\begin{array}{c}
\mathbf{p}^{(1)} \\
\mathbf{p}^{(2)} \\
\vdots \\
\mathbf{p}^{\left(n_{p}\right)}
\end{array}\right]=\left[\begin{array}{c}
\frac{1}{p_{\max }^{(1)}} \mathbf{p}^{(1)} \\
\frac{1}{p_{\max }^{(2)}} \mathbf{p}^{(2)} \\
\vdots \\
\frac{1}{p_{\max }^{\left(n_{p}\right)}} \mathbf{p}^{\left(n_{p}\right)}
\end{array}\right]
$$

where $S_{\mathrm{p}}$ is the normalization matrix, and

$$
p_{\max }^{(i)}=\max \left\{\left\|\mathbf{p}_{1}^{(i)}\right\|_{\infty},\left\|\mathbf{p}_{2}^{(i)}\right\|_{\infty}, \cdots,\left\|\mathbf{p}_{M}^{(i)}\right\|_{\infty}\right\}
$$

is the maximum value of the $i$-th type input parameters of the $M$ physical fields in the database $\mathbf{S}$. 
With the normalization, we can ignore the influence of the different units of various types of input parameters. Therefore, we can avoid the case that the influence of one type of input parameter overwhelms that of another type.

Next, we scale the random input-to-hidden layer connection weights. Considering Equation (3.25), we rewrite the input of the activation function as the following compact form

$$
\mathbf{z}=\mathbf{R p}+\varepsilon
$$

where $\mathbf{z}=\left[z_{1}, z_{2}, \cdots, z_{L}\right]^{\mathrm{T}}, \mathbf{R}=\left[\mathbf{r}_{1}, \mathbf{r}_{2}, \cdots, \mathbf{r}_{L}\right]^{\mathrm{T}}$ and $\boldsymbol{\varepsilon}=\left[\varepsilon_{1}, \varepsilon_{2}, \cdots, \varepsilon_{L}\right]^{\mathrm{T}}$.

Referring to Figure 3.3, we can find that for all $1 \leq i \leq L$, if $\left|z_{i}\right|>4$ the output of hidden layer neuron (see Figure 3.2) $g\left(z_{i}\right)=0$ or $g\left(z_{i}\right)=1$, which make the regression model cannot distinguish two different input parameters $\mathbf{p}$ and $\mathbf{p}^{\prime}$ if they both can lead to $\left|z_{i}\right|>4$ for all $1 \leq i \leq L$. One simple way to settle this issue is to scale the random weight matrix $\mathbf{R}$ and the random bias $\varepsilon$.

We simply set

$$
\mathbf{z}_{\mathrm{s}}=\xi \mathbf{z}=\xi \mathbf{R} \mathbf{p}_{\mathrm{s}}+\xi \varepsilon
$$

where

$$
\xi=\frac{4}{z_{\max }}
$$

and

$$
z_{\max }=\max \left\{\left\|\mathbf{z}^{(1)}\right\|_{\infty},\left\|\mathbf{z}^{(2)}\right\|_{\infty}, \cdots,\left\|\mathbf{z}^{(M)}\right\|_{\infty}\right\}
$$

Here,

$$
\mathbf{z}^{(i)}=\mathbf{R} \mathbf{p}_{\mathrm{s}}^{(i)}+\boldsymbol{\varepsilon}=\mathbf{R} S_{\mathrm{p}} \mathbf{p}_{i}+\boldsymbol{\varepsilon}
$$

for all $1 \leq i \leq M$.

Then, using $\mathbf{z}_{\mathrm{s}}$ instead of $\mathbf{z}$ for the standard ELM, we can obtain the output of th SLFN

$$
\mathbf{c}(\mathbf{p})=\sum_{i=1}^{L} \boldsymbol{\beta}_{i} g_{i}(\mathbf{p})=\sum_{i=1}^{L} \boldsymbol{\beta}_{i} g\left(\xi \mathbf{r}_{i}^{\mathrm{T}} S_{\mathrm{p}} \mathbf{p}+\xi \varepsilon_{i}\right)
$$

We call this method scaled extreme learning machine (sELM). 
The sELM is a variation of the standard ELM. It has the same neural network structure with the standard ELM. Comparing (3.19) and (3.27), we can find that the sELM is similar with the standard ELM but with scaled input parameters, random weights, and random bias. We can use the same way (Equations (3.20)(3.23) ) to train the sELM and reconstruct the physical field of interest, but use $g\left(\xi \mathbf{r}_{i}^{\mathrm{T}} S_{\mathrm{p}} \mathbf{p}_{j}+\xi \varepsilon_{i}\right)$ instead of $g_{i}\left(\mathbf{p}_{j}\right)$ in (3.21). Therefore, the sELM maintains the computational efficiency of the standard ELM. But the small changes on the standard ELM can resolve the aforementioned two issues and improve the performance.

Remark 5. The purpose of the first-stage is to estimate the physical field from the observed input parameters. The SLFN is a multi-input-multi-output regression model. ELM and sELM are two methods to train the model. Using ELM/sELM for the physical field reconstruction, we only train one regression model, while using the spline interpolation, we need to train $\check{n}$ regression models. Therefore, ELM/sELM can be more convenient alternatives of the spline interpolation technique for physical field reconstruction.

\subsubsection{Second-stage: physical field enhancement based on the sparse sensor observations}

As shown in Figure 3.1, in the second stage, we estimate the error of the reconstructed physical field in the first stage based on the sparse sensor observations. With the estimated error field, we can correct the physical field obtained in the first stage.

We introduce an error field e to represent the difference between the physical field $\mathbf{s}(\mathbf{p})$ and its estimation $\check{\mathbf{s}}(\mathbf{p})$ obtained from the first stage, i.e.,

$$
\mathbf{e}(\mathbf{p})=\mathbf{s}(\mathbf{p})-\check{\mathbf{s}}(\mathbf{p})
$$


We denote the difference between sensor observations and the estimated field $\check{\mathbf{s}}(\mathbf{p})$ at $m$ sensor locations by a vector $\mathbf{d}$. From (3.1) and (3.28), we can easily obtain

$$
\mathbf{d}=\mathbf{y}-\mathbf{H} \check{\mathbf{s}}(\mathbf{p})=\mathbf{H e}(\mathbf{p})+\mathbf{v}
$$

We assume that the noise of sensor observations is much smaller than the error of the estimated field, i.e., $|v(x)| \ll|e(x, \mathbf{p})|$ for all $x \in \mathbb{X}_{\mathrm{s}}$.

It is easily found from (3.2), (3.24), and (3.28) that

$$
\mathbf{e}(\mathbf{p})=\mathbf{w}(\mathbf{p})-\check{\mathbf{w}}(\mathbf{p})
$$

Both $\check{\mathbf{w}}(\mathbf{p})$ and $\mathbf{w}(\mathbf{p})$ can be approximated by a combination of dominant PCA modes, so too the error field e, i.e.,

$$
\mathbf{e}(\mathbf{p})=\tilde{\boldsymbol{\Phi}} \boldsymbol{\alpha}+\tilde{\boldsymbol{\Phi}}_{\text {tail }} \boldsymbol{\alpha}_{\text {tail }}
$$

where $\boldsymbol{\alpha}=\left[\alpha_{1}, \alpha_{2}, \ldots, \alpha_{n}\right]$ and $\boldsymbol{\alpha}_{\text {tail }}=\left[\alpha_{n+1}, \alpha_{n+2}, \ldots, \alpha_{N}\right]$ represent the coefficients of the dominant and subordinate PCA modes of the error field $\mathbf{e}(\mathbf{p})$, respectively.

Substituting (3.31) into (3.29) and ignoring the subdominant components yields

$$
\begin{aligned}
\mathbf{d} & =\mathbf{H} \tilde{\boldsymbol{\Phi}} \boldsymbol{\alpha}+\mathbf{H} \tilde{\boldsymbol{\Phi}}_{\text {tail }} \boldsymbol{\alpha}_{\text {tail }}+\mathbf{v} \\
& \approx \mathbf{H} \tilde{\boldsymbol{\Phi}} \boldsymbol{\alpha}+\mathbf{v} \\
& =\boldsymbol{\Phi} \boldsymbol{\alpha}+\mathbf{v}
\end{aligned}
$$

Using least-squares technique, from (3.32c) we can find the following estimate of $\alpha$

$$
\hat{\boldsymbol{\alpha}}=\boldsymbol{\Phi}^{\dagger} \mathbf{d}=\left(\boldsymbol{\Phi}^{\mathrm{T}} \boldsymbol{\Phi}\right)^{-1} \boldsymbol{\Phi}^{\mathrm{T}} \mathbf{d}
$$

with which the estimated error field is given by

$$
\hat{\mathbf{e}}(\mathbf{p})=\tilde{\Phi} \hat{\alpha}=\tilde{\Phi} \Phi^{\dagger} \mathbf{d}
$$


With the estimated error field, we can then enhance $\check{\mathbf{s}}(\mathbf{p})$ and obtain a more accurate estimation

$$
\begin{aligned}
\tilde{\mathbf{s}}(\mathbf{p}) & =\check{\mathbf{s}}(\mathbf{p})+\hat{\mathbf{e}}(\mathbf{p}) \\
& =\overline{\mathbf{s}}+\check{\boldsymbol{\Phi}} \check{\mathbf{c}}(\mathbf{p})+\tilde{\boldsymbol{\Phi}} \hat{\boldsymbol{\alpha}}
\end{aligned}
$$

In this equation, $\check{\boldsymbol{\Phi}} \check{\mathbf{c}}(\mathbf{p})$ includes all the involved PCA components and $\tilde{\boldsymbol{\Phi}} \hat{\boldsymbol{\alpha}}$ improves the estimation of the dominant PCA components.

Remark 6. In Equation (3.35), the second last term can guarantee a small truncation error, and the last term can correct the dominant PCA components in the second last term. Therefore, the proposed method owns both merits of the two current approaches detailed in Section 3.2.

\subsection{Error analysis}

To assess the performance of the proposed TSPFR method, we compare the errors of the reconstructed physical fields obtained from the TSPFR and the two current approaches discussed in Section 3.2.

\subsubsection{Error of the physical field estimated from the ob- served input parameters}

Firstly, we consider the physical field estimated from the input parameters with regression models. Since $\check{n}$ is large enough, the truncation error can be ignored. The error of the reconstructed field fully depends on the error of the estimated PCA coefficients.

As shown in (3.15) and (3.23), the accuracy of PCA coefficients depends on the regression model and the accuracy of the observed input parameters. The accuracy 
of the observed input parameters depends on the sensors. Training a good regression model (via spline interpolation or sELM) usually requires a large number of accurate physical field samples in the database $\mathbf{S}$. The more the number of samples, the better is the regression model. However, as discussed in Chapter 2, obtain one accurate physical field is usually very computational expensive. Therefore, for some applications, it is not easy to obtain a very good regression model with which the accuracy of the reconstructed physical field may not meet our requirement. However, the estimated PCA coefficients must satisfy that

$$
\left|\check{c}_{k}-c_{k}\right|<\left|c_{k}\right|
$$

for all $k \in \mathcal{I}_{\overline{0}}$. Otherwise the estimated PCA coefficients from regression model will be totally meaningless, and we can use 0 instead of the corresponding estimated PCA coefficients.

\subsubsection{Error of the physical field estimated from sparse sen- sor observations}

In this part, we analyze the error of $\hat{\mathbf{s}}_{n}$, which is the physical field estimated from the sparse sensor observations as discussed in Section 3.2.3.

Substituting (3.8) into (3.1) yields

$$
\mathbf{y}=\mathbf{H} \overline{\mathbf{s}}+\boldsymbol{\Phi} \mathbf{c}+\boldsymbol{\Phi}_{\text {tail }} \mathbf{c}_{\text {tail }}+\mathbf{v}
$$

where $\boldsymbol{\Phi}_{\text {tail }}=\mathbf{H} \tilde{\boldsymbol{\Phi}}_{\text {tail }} \in \mathbb{R}^{m \times(N-n)}$, similar to $\boldsymbol{\Phi}$, is a pruned matrix that contains the rows of $\boldsymbol{\Phi}_{\text {tail }}$ indexed by $\mathcal{L}=\left\{l_{1}, l_{2}, \ldots, l_{m}\right\}$. Unlike (3.16), Equation (3.36) provides an exact decomposition of sensor observations.

Then, substituting (3.36) into (3.17) yields

$$
\hat{\mathbf{c}}=\mathbf{c}+\boldsymbol{\Phi}^{\dagger} \boldsymbol{\Phi}_{\text {tail }} \mathbf{c}_{\text {tail }}+\boldsymbol{\Phi}^{\dagger} \mathbf{v}
$$


We define the error energy of $\hat{\mathbf{s}}_{n}$ in $(3.18)$ as

$$
\hat{E}_{n}:=\left\|\mathbf{s}-\hat{\mathbf{s}}_{n}\right\|_{2}^{2}
$$

Substituting (3.8) and (3.18) into (3.38), we have

$$
\begin{aligned}
\hat{E}_{n} & =\|\mathbf{w}-\tilde{\boldsymbol{\Phi}} \hat{\mathbf{c}}\|_{2}^{2} \\
& =\|\mathbf{w}-\tilde{\boldsymbol{\Phi}} \mathbf{c}+\tilde{\boldsymbol{\Phi}} \mathbf{c}-\tilde{\boldsymbol{\Phi}} \hat{\mathbf{c}}\|_{2}^{2} \\
& =\left\|\tilde{\boldsymbol{\Phi}}_{\text {tail }} \mathbf{c}_{\text {tail }}+\tilde{\boldsymbol{\Phi}}(\mathbf{c}-\hat{\mathbf{c}})\right\|_{2}^{2}
\end{aligned}
$$

It is clear that $\tilde{\boldsymbol{\Phi}}_{\text {tail }} \mathbf{c}_{\text {tail }} \in \operatorname{span}\left\{\boldsymbol{\varphi}_{n+1}, \boldsymbol{\varphi}_{n+2}, \ldots, \boldsymbol{\varphi}_{N}\right\}$ and $\tilde{\boldsymbol{\Phi}}(\mathbf{c}-\hat{\mathbf{c}}) \in \operatorname{span}\left\{\boldsymbol{\varphi}_{1}, \boldsymbol{\varphi}_{2}, \ldots, \boldsymbol{\varphi}_{n}\right\}$, which implies that the two vectors are orthogonal with each other, i.e., $\tilde{\boldsymbol{\Phi}}_{\text {tail }} \mathbf{c}_{\text {tail }} \perp$ $\tilde{\boldsymbol{\Phi}}(\mathbf{c}-\hat{\mathbf{c}})$. In addition, considering both $\tilde{\boldsymbol{\Phi}}$ and $\tilde{\boldsymbol{\Phi}}_{\text {tail }}$ are sub-unitary matrices, we can obtain

$$
\begin{aligned}
\hat{E}_{n} & =\left\|\tilde{\boldsymbol{\Phi}}_{\text {tail }} \mathbf{c}_{\text {tail }}\right\|_{2}^{2}+\|\tilde{\boldsymbol{\Phi}}(\mathbf{c}-\hat{\mathbf{c}})\|_{2}^{2} \\
& =\left\|\mathbf{c}_{\text {tail }}\right\|_{2}^{2}+\|\mathbf{c}-\hat{\mathbf{c}}\|_{2}^{2}
\end{aligned}
$$

Substituting (3.37) into (3.39b) yields

$$
\begin{aligned}
\hat{E}_{n} & =\left\|\mathbf{c}_{\text {tail }}\right\|_{2}^{2}+\left\|\boldsymbol{\Phi}^{\dagger} \boldsymbol{\Phi}_{\text {tail }} \mathbf{c}_{\text {tail }}+\boldsymbol{\Phi}^{\dagger} \mathbf{v}\right\|_{2}^{2} \\
& \leq\left\|\mathbf{c}_{\text {tail }}\right\|_{2}^{2}+\left\|\boldsymbol{\Phi}^{\dagger} \boldsymbol{\Phi}_{\text {tail }}\right\|_{2}^{2}\left\|\mathbf{c}_{\text {tail }}\right\|_{2}^{2}+\left\|\boldsymbol{\Phi}^{\dagger}\right\|_{2}^{2}\|\mathbf{v}\|_{2}^{2}
\end{aligned}
$$

\subsubsection{Error of the physical field estimated via TSPFR}

In this part, we consider the error of $\tilde{\mathbf{s}}$, i.e., the physical field estimated from the proposed TSPFR approach.

Substituting (3.32a) into (3.33) yields

$$
\hat{\boldsymbol{\alpha}}=\boldsymbol{\alpha}+\boldsymbol{\Phi}^{\dagger} \boldsymbol{\Phi}_{\text {tail }} \boldsymbol{\alpha}_{\text {tail }}+\boldsymbol{\Phi}^{\dagger} \mathbf{v}
$$


Similarly, we introduce the error energy of the enhanced physical field $\tilde{\mathbf{s}}$ in (3.35) as

$$
\tilde{E}:=\|\mathbf{s}-\tilde{\mathbf{s}}\|_{2}^{2}
$$

Substituting (3.28) and (3.35) into (3.42) yields

$$
\tilde{E}=\left\|\mathbf{s}-\hat{\mathbf{s}}-\hat{\mathbf{e}}_{n}\right\|_{2}^{2}=\left\|\mathbf{e}-\hat{\mathbf{e}}_{n}\right\|_{2}^{2}
$$

Substituting (3.31) and (3.34) into (3.43) yields

$$
\tilde{E}=\left\|\tilde{\boldsymbol{\Phi}}_{\text {tail }} \boldsymbol{\alpha}_{\text {tail }}+\tilde{\boldsymbol{\Phi}}(\boldsymbol{\alpha}-\hat{\boldsymbol{\alpha}})\right\|_{2}^{2}
$$

Since $\tilde{\boldsymbol{\Phi}}_{\text {tail }} \boldsymbol{\alpha}_{\text {tail }}$ and $\tilde{\boldsymbol{\Phi}}(\boldsymbol{\alpha}-\hat{\boldsymbol{\alpha}})$ are orthogonal,

$$
\tilde{E}=\left\|\boldsymbol{\alpha}_{\text {tail }}\right\|_{2}^{2}+\|\boldsymbol{\alpha}-\hat{\boldsymbol{\alpha}}\|_{2}^{2}
$$

Replacing $\hat{\boldsymbol{\alpha}}$ by the right hand side of (3.41), we can obtain

$$
\begin{aligned}
\tilde{E} & =\left\|\boldsymbol{\alpha}_{\text {tail }}\right\|_{2}^{2}+\left\|\boldsymbol{\Phi}^{\dagger} \boldsymbol{\Phi}_{\text {tail }} \boldsymbol{\alpha}_{\text {tail }}+\boldsymbol{\Phi}^{\dagger} \mathbf{v}\right\|_{2}^{2} \\
& \leq\left\|\boldsymbol{\alpha}_{\text {tail }}\right\|_{2}^{2}+\left\|\boldsymbol{\Phi}^{\dagger} \boldsymbol{\Phi}_{\text {tail }}\right\|_{2}^{2}\left\|\boldsymbol{\alpha}_{\text {tail }}\right\|_{2}^{2}+\left\|\boldsymbol{\Phi}^{\dagger}\right\|_{2}^{2}\|\mathbf{v}\|_{2}^{2}
\end{aligned}
$$

\subsubsection{Error comparison}

The accuracy of the physical fields estimated from the observed input parameters via a regression model depends on the quality of the regression model. It is not easy to theoretically compare the error of the reconstructed field $\check{\mathbf{s}}$ with the error of $\hat{\mathbf{s}}_{n}$ and that of $\tilde{\mathbf{s}}$. Later, we provide the error comparisons of them via an example. Next, we compare the error energy of $\hat{\mathbf{s}}_{n}$ and that of $\tilde{\mathbf{s}}$. Clearly, both $\boldsymbol{\Phi}^{\dagger}$ and $\boldsymbol{\Phi}_{\text {tail }}$ depend on the database $\mathbf{S}$ and sensor locations. Considering (3.40a) and (3.44a), if the database and the sensors associated with their locations are fixed, $\hat{E}_{n}$ and $\tilde{E}$ only depend on $\mathbf{c}_{\text {tail }}$ and $\boldsymbol{\alpha}_{\text {tail }}$, respectively. 
Considering the definition of PCA coefficients, from (3.30) and (3.34), we can obtain

$$
\boldsymbol{\alpha}_{\text {tail }}=\tilde{\boldsymbol{\Phi}}_{\text {tail }}^{\mathrm{T}} \mathbf{e}=\tilde{\boldsymbol{\Phi}}_{\text {tail }}^{\mathrm{T}}(\mathbf{w}-\check{\mathbf{w}})=\mathbf{c}_{\text {tail }}-\tilde{\boldsymbol{\Phi}}_{\text {tail }}^{\mathrm{T}} \check{\boldsymbol{\Phi}} \check{\mathbf{c}}
$$

If $\check{n}=n$, we have $\check{\boldsymbol{\Phi}}=\tilde{\boldsymbol{\Phi}}$ and $\tilde{\boldsymbol{\Phi}}_{\text {tail }}^{\mathrm{T}} \check{\boldsymbol{\Phi}}=\mathbf{0}$, where $\mathbf{0}$ is a zero matrix with proper dimension. In that case, $\boldsymbol{\alpha}_{\text {tail }}=\mathbf{c}_{\text {tail }}$ and therefore $\hat{E}_{n}=\tilde{E}$. In this case $\hat{\mathbf{s}}_{n}=\tilde{\mathbf{s}}$.

In practice, however, $\check{n}$ is usually larger than $n$. Since $\left|c_{k}(\mathbf{p})\right|<\varepsilon \approx 0$ for all $\mathbf{p} \in \mathbb{P}_{\mathrm{s}}$ and $k>\check{n}$, we can assume that

$$
\mathbf{c}_{\text {tail }}=\left[\begin{array}{ll}
\mathbf{c}_{n+1: \check{n}}^{\mathrm{T}} & \mathbf{0}^{\mathrm{T}}
\end{array}\right]^{\mathrm{T}}
$$

where $\mathbf{c}_{n+1: \check{n}}=\left[\begin{array}{lll}c_{n+1} & c_{n+2} \ldots c_{\check{n}}\end{array}\right]^{\mathrm{T}}$. Let $\check{\mathbf{c}}=\left[\begin{array}{ll}\check{\mathbf{c}}_{1: n}^{\mathrm{T}} & \check{\mathbf{c}}_{n+1: \check{n}}^{\mathrm{T}}\end{array}\right]^{\mathrm{T}}$ where $\check{\mathbf{c}}_{1: n}=\left[\begin{array}{lll}\check{c}_{1} & \check{c}_{2} \ldots \check{c}_{n}\end{array}\right]$ and $\check{\mathbf{c}}_{n+1: \check{n}}=\left[\check{c}_{n+1} \check{c}_{n+2} \ldots \check{c}_{\check{n}}\right]$. Since $\check{\boldsymbol{\Phi}}=\left[\begin{array}{ll}\tilde{\boldsymbol{\Phi}} & \tilde{\boldsymbol{\Phi}}_{n+1: \check{n}}\end{array}\right]$ and $\tilde{\boldsymbol{\Phi}}_{n+1: \check{n}}=\left[\boldsymbol{\varphi}_{n+1}, \boldsymbol{\varphi}_{n+2}, \ldots, \boldsymbol{\varphi}_{\check{n}}\right]$, we have

$$
\boldsymbol{\Phi}_{\text {tail }}^{\mathrm{T}} \check{\boldsymbol{\Phi}}=\left[\begin{array}{cc}
\mathbf{0} & \mathbf{I}_{\check{n}-n} \\
\mathbf{0} & \mathbf{0}
\end{array}\right]
$$

Then, from (3.45) we can obtain

$$
\boldsymbol{\alpha}_{\text {tail }}=\left[\begin{array}{ll}
\mathbf{c}_{n+1: \check{n}}^{\mathrm{T}} & \mathbf{0}^{\mathrm{T}}
\end{array}\right]^{\mathrm{T}}-\left[\check{\mathbf{c}}_{n+1: \check{n}}^{\mathrm{T}} \mathbf{0}^{\mathrm{T}}\right]^{\mathrm{T}}
$$

It is obvious that

$$
\begin{aligned}
\left\|\boldsymbol{\alpha}_{\text {tail }}\right\|_{2}^{2} & =\left\|\mathbf{c}_{n+1: \check{n}}-\check{\mathbf{c}}_{n+1: \check{n}}\right\|_{2}^{2} \\
\left\|\mathbf{c}_{\text {tail }}\right\|_{2}^{2} & =\left\|\mathbf{c}_{n+1: \check{n}}\right\|_{2}^{2}
\end{aligned}
$$

Since $\left|\check{c}_{k}-c_{k}\right|<\left|c_{k}\right|$ for all $k \in \mathcal{I}_{\overline{0}}$ we can obtain

$$
\left\|\mathbf{c}_{n+1: \check{n}}-\check{\mathbf{c}}_{n+1: \check{n}}\right\|_{2}^{2}<\left\|\mathbf{c}_{n+1: \check{n}}\right\|_{2}^{2}
$$

Hence,

$$
\left\|\boldsymbol{\alpha}_{\text {tail }}\right\|_{2}^{2}<\left\|\mathbf{c}_{\text {tail }}\right\|_{2}^{2}
$$


which implies that $\tilde{E}<\hat{E}_{n}$, and the proposed TSPFR approach outperforms the physical field reconstruction directly from the sparse sensor observations via PCA.

\subsection{Sensor placement}

From Equations (3.40b) and (3.44b), we can find that the error energy $\hat{E}_{n}$ and $\tilde{E}$ depend on:

1. the subdominant components (i.e., $\mathbf{c}_{\text {tail }}$ and $\boldsymbol{\alpha}_{\text {tail }}$ perspectively),

2. the noise level of sensor observations

3. the norm of the matrices $\boldsymbol{\Phi}^{\dagger} \boldsymbol{\Phi}_{\text {tail }}$ and $\boldsymbol{\Phi}^{\dagger}$.

The subdominant components depends on the truncation level $n$ which is limited by the number of sensor observations $m$. Therefore, if the sensors are given, the truncation level would be bounded and the noise level of their observations would be fixed.

For the proposed TSPFR approach, except the number of sensor observations and their noise level, as can be seen from Equation (3.46), $\boldsymbol{\alpha}_{\text {tail }}$ is also related to $\check{\mathbf{c}}_{n+1: \check{n}}$, which is estimated from the regression model. Hence, $\boldsymbol{\alpha}_{\text {tail }}$ also depends on the noise level of the observed input parameters $\mathbf{p}_{\mathrm{o}}$ and accuracy of the regression model.

$\boldsymbol{\Phi}$ and $\boldsymbol{\Phi}_{\text {tail }}$ are pruned matrices from some rows of $\tilde{\boldsymbol{\Phi}}$ and $\tilde{\boldsymbol{\Phi}}_{\text {tail }}$ indexed by $\mathcal{L}=$ $\left\{l_{1}, l_{2}, \ldots, l_{m}\right\}$, respectively. The number of rows of $\boldsymbol{\Phi}$ and $\boldsymbol{\Phi}_{\text {tail }}$ (i.e., $m$ ) is limited by the number sensor observations, and the row indices corresponds to the sensing locations. Both the number of sensors and their sensing locations are designable. We can determine the minimum required sensor nodes and select their sensing positions to minimize $\left\|\boldsymbol{\Phi}^{\dagger} \boldsymbol{\Phi}_{\text {tail }}\right\|_{2}^{2}$ and $\left\|\boldsymbol{\Phi}^{\dagger}\right\|_{2}^{2}$. This is a combinatorial optimization problem and it is typical NP-hard. We focus on this problem in Chapter 
4, and provide a new method named maximal projection on minimum eigenspace (MPME).

\subsection{Number reduction of the sensor observations}

In practice, the sensor observations of one physical field may be expensive. For some applications, it is impractical to set many sensors for indoor environment. It will be very interesting to reduce the number of sensor observations but preserve the estimation performance.

If the number of sensor observations is reduced, one direct way for physical field reconstruction is to reduce the truncation level, i.e., reduce the number of dominant PCA modes. On one hand, this action would increase the truncation error (both $\mathbf{c}_{\text {tail }}$ and $\boldsymbol{\alpha}_{\text {tail }}$ ). On the other hand, Equations (3.37) and (3.41) indicate that a larger truncation error would make the estimation of the dominant PCA coefficients worse. For the proposed TSPFR approach, however, it is possible to reduce the number of dominant PCA modes and guarantee performance of the physical field estimation.

The Equation (3.35) can be rewritten as

$$
\tilde{\mathbf{s}}(\mathbf{p})=\overline{\mathbf{s}}+\tilde{\boldsymbol{\Phi}}\left(\check{\mathbf{c}}_{1: n}(\mathbf{p})+\hat{\boldsymbol{\alpha}}\right)+\check{\boldsymbol{\Phi}}_{n+1: \check{n}} \check{\mathbf{c}}_{n+1: \check{n}}(\mathbf{p})
$$

where $\check{\mathbf{c}}_{1: n}=\left[\check{c}_{1}, \check{c}_{2}, \cdots, \check{c}_{n}\right]^{\mathrm{T}}, \check{\mathbf{\Phi}}_{n+1: \check{n}}=\left[\boldsymbol{\varphi}_{n+1}, \boldsymbol{\varphi}_{n+1}, \cdots, \boldsymbol{\varphi}_{\check{n}}\right]$, and $\check{\mathbf{c}}_{n+1: \check{n}}=\left[\check{c}_{n+1}\right.$, $\left.\check{c}_{n+2}, \cdots, \check{c}_{\check{n}}\right]^{\mathrm{T}} . \tilde{\boldsymbol{\Phi}}\left(\check{\mathbf{c}}_{1: n}(\mathbf{p})+\hat{\boldsymbol{\alpha}}\right)$ represents the dominant PCA components of $\tilde{\mathbf{s}}(\mathbf{p})$ and $\check{\mathbf{\Phi}}_{n+1: \check{n}} \check{\mathbf{c}}_{n+1: \check{n}}(\mathbf{p})$ represents the estimated subdominant PCA components. It is clear that $\hat{\boldsymbol{\alpha}}$ corrects the dominant PCA coefficients estimated from the first stage.

In the second stage, we assume that the error of the physical field estimated in the first stage and the physical field itself have the same dominant PCA modes. In this way, the truncation error of the error field is very small, and from the sensor observations we can well estimate the dominant PCA coefficients of the 
error field, with which we can correct all dominant PCA coefficients of the physical field estimated in the first stage. However, some 'dominant components' of the error field may be insignificant.

In the second stage, $\boldsymbol{\alpha}$ is viewed as the dominant PCA coefficients of the error field. If some dominant PCA components of the physical field are well-estimated in the first stage, the corresponding PCA components of the error field will be very small and insignificant. In other words, if some entry of $\check{\mathbf{c}}_{1: n}=\left[\check{c}_{1}, \check{c}_{2}, \cdots, \check{c}_{n}\right]^{\mathrm{T}}$, say $\check{c}_{i}, i \leq n$, is well-estimated, the corresponding PCA coefficient of the error field $\alpha_{i}$ would be very small. We can approximate $\alpha_{i}$ as zero. In that case, $\alpha_{i}$ is not required to be estimated and $\check{c}_{i}$ is not required to be corrected in the second stage.

If we know the indices of dominant PCA coefficients that are well-estimated in the first stage, we can remove the corresponding PCA modes from $\mathbf{\Phi}$ and modify Equation (3.32c) as

$$
\mathbf{d} \approx \boldsymbol{\Phi} \boldsymbol{\alpha}+\mathbf{v} \approx \Phi_{\mathrm{r}} \boldsymbol{\alpha}_{\mathrm{r}}+\mathbf{v}=\mathbf{H} \tilde{\Phi}_{r} \boldsymbol{\alpha}_{\mathrm{r}}+\mathbf{v}
$$

where $\tilde{\boldsymbol{\Phi}}_{\mathrm{r}}=\left[\boldsymbol{\varphi}_{r_{1}}, \boldsymbol{\varphi}_{r_{2}}, \cdots, \boldsymbol{\varphi}_{r_{n_{r}}}\right], \boldsymbol{\alpha}_{\mathrm{r}}=\left[\alpha_{r_{1}}, \alpha_{r_{2}}, \cdots, \alpha_{r_{r}}\right]^{\mathrm{T}}, n_{r} \leq n$, and the index set

$$
\mathcal{R}=\left\{r_{1}, r_{2}, \cdots, r_{n_{r}}\right\} \subseteq\{1,2, \cdots, n\} .
$$

Since $\boldsymbol{\alpha}_{\mathrm{r}} \in \mathbb{R}^{n_{r}}$, we can estimate $\boldsymbol{\alpha}_{\mathrm{r}}$ with only $n_{r}$ sensor observations. Since $n_{r} \leq n$, the minimum required number of sensor observations can be reduced from $n$ to $n_{r}$. We can reduce the number of sensor observations and formulate the new measurement equation of the error field as

$$
\mathbf{d}_{\mathrm{r}} \approx \mathbf{H}_{\mathrm{r}} \tilde{\boldsymbol{\Phi}}_{r} \boldsymbol{\alpha}_{\mathrm{r}}+\mathbf{v}_{r}
$$

where $\mathbf{d}_{r} \in \mathbb{R}^{m_{r}}$ represents the sensor observations, $\mathbf{v}_{r} \in \mathbb{R}^{m_{r}}$ represents the measurement noise, $m_{r}$ is the number of sensor observations and $n_{r} \leq m_{r} \leq m$. Here, $\mathbf{H}_{\mathrm{r}} \in \mathbb{R}^{m_{r} \times N}$ is the sampling kernel which consists of $m_{r}$ rows of the identify matrix I index by $\mathcal{L}^{\prime}=\left\{l_{1}^{\prime}, l_{2}^{\prime}, \cdots, l_{m_{r}}^{\prime}\right\}$. The index set $\mathcal{L}^{\prime}$ represents the sensing locations. 
This set can be determined by solving the optimization problem (4.8) in which $\tilde{\boldsymbol{\Phi}}$ and $\mathcal{L}$ are replaced by $\tilde{\boldsymbol{\Phi}}_{r}$ and $\mathcal{L}^{\prime}$, respectively.

The index set $\mathcal{R}$ includes the indices of the dominant PCA coefficients estimated in the first stage, and these coefficients are not accurate enough. On the contrary, the dominant PCA coefficients whose indices are not in $\mathcal{R}$ are well-estimated in the first stage, and they are not require to be corrected. Therefore, in the second stage, we only use the PCA modes with indices in $\mathcal{R}$. With the index set $\mathcal{R}$, we can easily find the matrix $\tilde{\boldsymbol{\Phi}}_{r}$. Then, we can reduce the number of sensor observations, which can even be reduced to $n_{r}(<n)$. If the number of available sensors is prior given and less than $n$, the key issue to achieve a good estimation is to determine the index set $\mathcal{R}$.

As we analyzed in Section 3.4.1, the accuracy of PCA coefficients estimated in the first stage depends on the regression model and the measurement noise of the observed input parameters. Ignoring the measurement noise of the input parameters, the estimation performance of the PCA coefficients mainly depends on the regression model.

With any regression model, the accuracy of one PCA coefficient estimated in the first stage may be greatly different with that of another PCA coefficient. We can use the cross validation technique to judge which PCA coefficient can be relatively well-estimated from a given regression model. In this work, for the cross validation technique, we use the leave-one out cross validation (LOOCV) [98]

Using any machine learning algorithm (i.e., spline interpolation, standard ELM, sELM or any other machine learning technique), we train $M$ regression models. For each regression model, we use $M-1$ physical fields in the database $\mathbf{S}$ to train the model, and use the other physical field to test the performance of the regression model. For $i$-th regression model, we use the $i$-th physical field in $\mathbf{S}$ for the testing. We denote the error of the PCA coefficients estimated in the first stage by

$$
\boldsymbol{\alpha}_{1: \check{n}}\left(\mathbf{p}_{i}\right)=\check{\mathbf{c}}\left(\mathbf{p}_{i}\right)-\mathbf{c}_{1: \check{n}}\left(\mathbf{p}_{i}\right), \quad 1 \leq i \leq M
$$


where $\check{\mathbf{c}}\left(\mathbf{p}_{i}\right)$ is the PCA coefficients estimated from the $i$-th regression model, and

$$
\mathbf{c}_{1: \check{n}}\left(\mathbf{p}_{i}\right)=\left[c_{1}\left(\mathbf{p}_{i}\right), c_{1}\left(\mathbf{p}_{i}\right), \cdots, c_{\check{n}}\left(\mathbf{p}_{i}\right)\right]^{\mathrm{T}}
$$

consists of the first $\check{n}$ PCA coefficients of the $i$-th physical field, which can be obtained from Equation (3.3).

Next, we introduce the mean absolute error (mAE) of the estimated PCA coefficients

$$
\operatorname{mAE}\left(\check{\mathbf{c}}\left(\mathbf{p}_{i}\right)\right)=\frac{1}{M} \sum_{i=1}^{M}\left|\boldsymbol{\alpha}_{1: \check{n}}\left(\mathbf{p}_{i}\right)\right|
$$

and the mAE of the estimated $k$-th PCA coefficient is

$$
\operatorname{mAE}\left(\check{c}_{k}\left(\mathbf{p}_{i}\right)\right)=\frac{1}{M} \sum_{i=1}^{M}\left|\alpha_{k}\left(\mathbf{p}_{i}\right)\right|
$$

With the mAE of all the involved PCA coefficients, we can judge the performance of the regression models trained by one machine learning algorithm (i.e., spline interpolation, standard ELM, sELM or any other machine learning technique) on each PCA coefficients. If the mAE of some PCA coefficients are very small, in the second stage we can ignore these PCA coefficients and only consider the other PCA coefficients. In that case, the number of sensor observations can be reduced.

Remark 7. Since the input-to-hidden layer weights are randomly generated when using ELM/sELM to train a regression model, in the LOOCV procedure, to remove the influence of the randomness we set the same input-to-hidden layer weights for all $M$ regression models.

\subsection{Example: indoor thermal map reconstruc- tion}

In Chapter 2, we provided a way to calibrate a simulated physical field with sparse sensor observations. In the example in Chapter 2, if the parameters of boundary 
condition change, e.g., the temperature of the inlet cooling air changes, and the power of the heaters changes, etc., we need to redo the CFD simulation and recalibrate the simulated physical field, which is tedious. While with the proposed TSPFR method, we can directly estimate the physical field from sparse sensor observations without redoing CFD simulations when the parameters of boundary condition changes. In this section, we use the example in Chapter 2 to test the performance of the proposed method. Again, we are interested in the thermal map $1.2 \mathrm{~m}$ above the ground of the room.

\subsubsection{Thermal map database}

The geometry description of this example have been given in Chapter 2. We have three type of heaters: 200W, 300W, and 400W. We have two of each type, and all the six heaters have the same size $(0.76 m \times 1.2 m \times 0.02 m)$. In Chapter 2 , we only use the heaters of $300 \mathrm{~W}$ and $400 \mathrm{~W}$. In this example, we also use four of them but some of the four may be turned off. Based on the location of the heaters, as shown in Figure 3.4, we denote the four heaters by heater 1, heater 2, heater 3, and heater 4 .

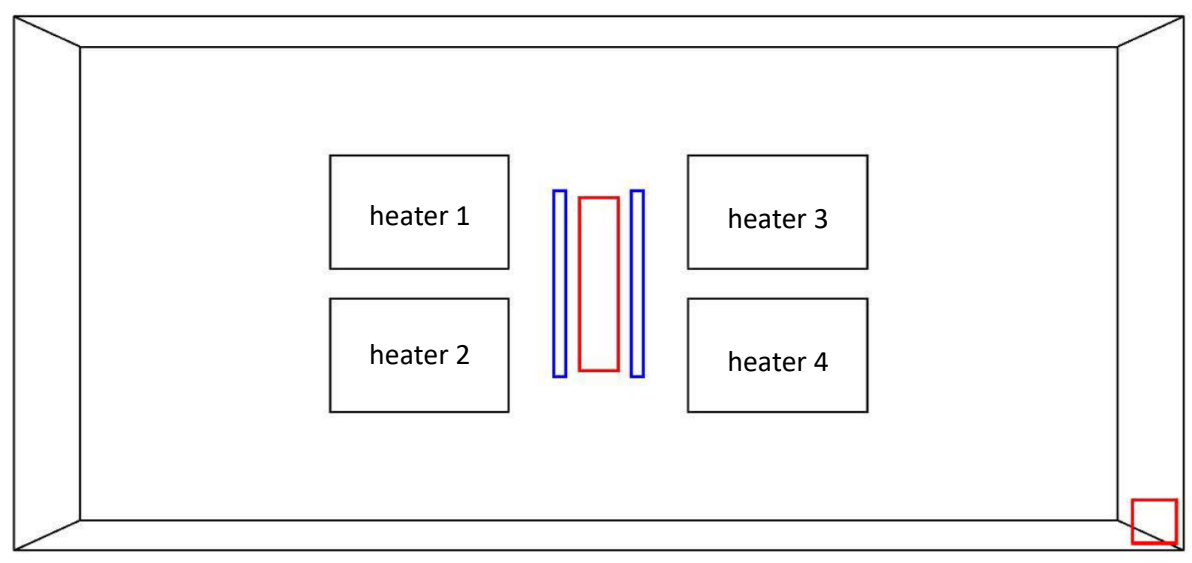

FiguRE 3.4: Top view sketch map of the room and the four heaters. 
In this example, we are interested in the change of the temperature of inlet cooling air and that of the power of the four heaters. The varying input parameters

$$
\mathbf{p}=\left[\begin{array}{lllll}
T_{\mathrm{in}} & P_{\mathrm{H} 1} & P_{\mathrm{H} 2} & P_{\mathrm{H} 3} & P_{\mathrm{H} 4}
\end{array}\right]^{\mathrm{T}}
$$

where $T_{\text {in }}$ is the temperature of the inlet cooling air, and $P_{\mathrm{H} i}$ is the power of the $i$-th heater. The range of the interest of $T_{\text {in }}$ is $[17.5,18.5]^{\circ} \mathrm{C}$. We sample it as $\{17.7,18,18.3\}^{\circ} \mathrm{C}$. Each heater may have four states: 0, 200W, 300W, and 400W. To reduce the workload in building the physical field database, we control the states of the four heaters and guarantee them satisfying the two constraints:

1. If both heater 1 and heater 2 are turned on, heater 1 and heater 2 are with the same power.

2. If both heater 3 and heater 4 are turned on, heater 3 and heater 4 are with the same power.

If only one heater is turned on, we have $4 \times 3 \times 3=36$ cases. For example, $\mathbf{p}_{1}=\left[\begin{array}{lllll}17.7^{\circ} \mathrm{C} & 200 \mathrm{~W} & 0 & 0 & 0\end{array}\right]^{\mathrm{T}}$ is one case of them. If two heaters are turned on, we have 18 cases. One example is $\mathbf{p}_{37}=\left[\begin{array}{lllll}17.7^{\circ} \mathrm{C} & 200 \mathrm{~W} & 200 \mathrm{~W} & 0 & 0\end{array}\right]^{\mathrm{T}}$. If four heaters are turned on, we also have 18 cases. One example is $\mathbf{p}_{55}=$ $\left[17.7^{\circ} \mathrm{C} \quad 200 \mathrm{~W} \quad 200 \mathrm{~W} \quad 300 \mathrm{~W} \quad 300 \mathrm{~W}\right]^{\mathrm{T}}$. For the 72 cases, from CFD simulations we can obtained 72 thermal maps (temperature fields) of the plane $1.2 \mathrm{~m}$ above the ground.

In the $72 \mathrm{CFD}$ simulations, the velocity of the inlet cooling air is set as $1.6 \mathrm{~m} / \mathrm{s}$. Except the varying input parameters, all the other settings of the CFD simulation is the same with the example in Chapter 2, which can be found in Table 2.1. The 72 thermal maps constitute the database $\mathbf{S}$. For the database $\mathbf{S}$, we did not consider the case that three heaters were turned on. Later, we will reconstruct a thermal map with three heaters on to show the effectiveness of the TSPFR. 


\subsubsection{The PCA modes}

With the thermal map database $\mathbf{S}$, we can easily find the PCA modes using the method of snapshot. The percentage energy and cumulative energy content for the PCA modes are shown in Figure 3.5. The exact values are given in Table 3.1.

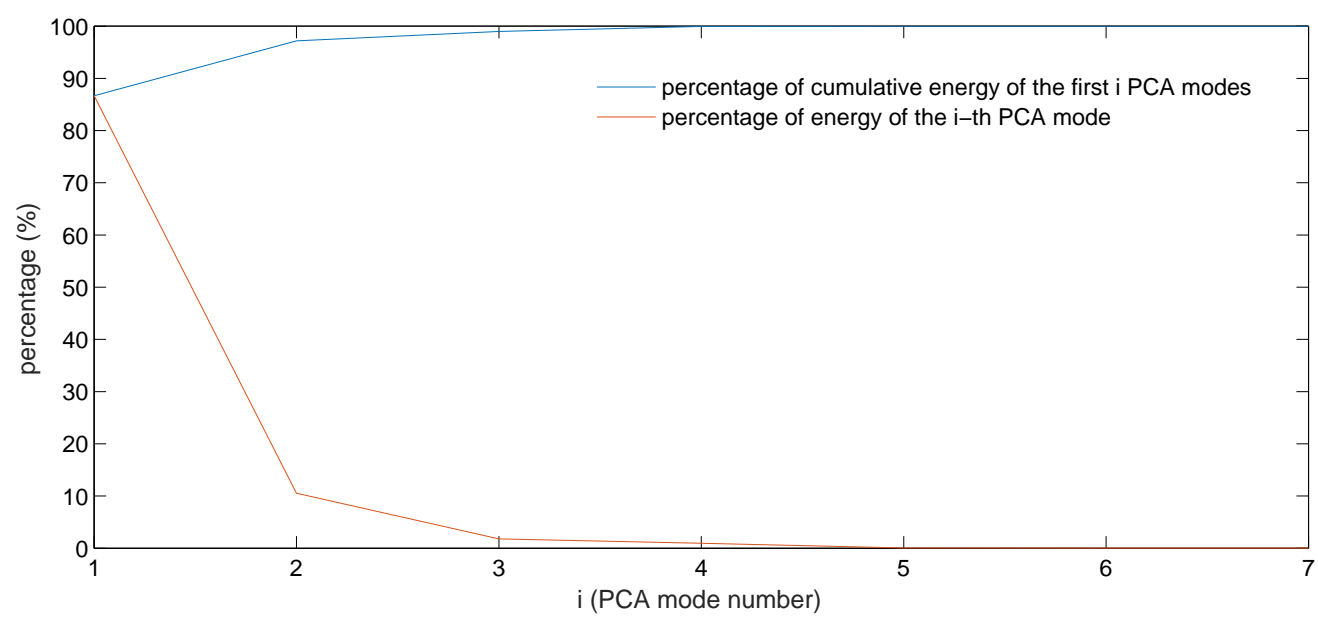

Figure 3.5: Percentage energy of the PCA modes

TABle 3.1: Percentage energy and cumulative energy content for PCA modes

\begin{tabular}{ccc}
\hline \hline Mode number $(i)$ & $\frac{100 \mu_{i}}{\sum_{k=1}^{72} \mu_{k}}(\%)$ & $\frac{100 \sum_{k=1}^{i} \mu_{k}}{\sum_{k=1}^{72} \mu_{k}}(\%)$ \\
\hline 1 & 86.669 & 86.669 \\
2 & 97.204 & 10.535 \\
3 & 98.996 & 1.792 \\
4 & 99.958 & 0.962 \\
5 & 99.998 & 0.040 \\
6 & 99.999 & 0.0006 \\
\hline \hline
\end{tabular}

It is clear that the first 4 PCA modes account for more than $99.9 \%$ energy of the thermal maps in the database $\mathbf{S}$. Therefore, we set the truncation level $n=4$. All the eighth PCA coefficients of the 72 thermal map in database $\mathbf{S}$ is less than 0.1 , which can be ignore compared with the first seven PCA coefficients of them. if we set $\varepsilon=0.1$, we can find that $\check{n}=7$. In the first stage, we reconstruct the thermal map by using the estimated first seven PCA coefficients. 


\subsubsection{Results and discussions}

Using the current one stage approaches and the proposed TSPFR approach, we reconstruct a thermal map from the observed input parameters and four temperature sensor observations of the thermal map.

For the input parameters, only the temperature of inlet cooling air is required to be observed. The observed $T_{\text {in }}=17.9^{\circ} \mathrm{C}$. The ground truth is a simulated thermal map obtained from the CFD simulation with input parameters $\mathbf{p}_{\mathrm{o}}=\left[17.9^{\circ} \mathrm{C} 200 \mathrm{~W}\right.$ 200W $0300 \mathrm{~W}$. The ground truth, i.e., the thermal map $1.2 \mathrm{~m}$ above the ground of the thermal lab in Figure 2.2, is shown in Figure 3.6.

The observed input parameters and four sensor observations are used to estimate the thermal map. The sensor noise is assumed to be independent identically distributed $\mathcal{N}\left(0,0.01^{2}\right)$ random variables. The sensing locations are determined by using the MPME algorithm, which is developed in Chapter 4.

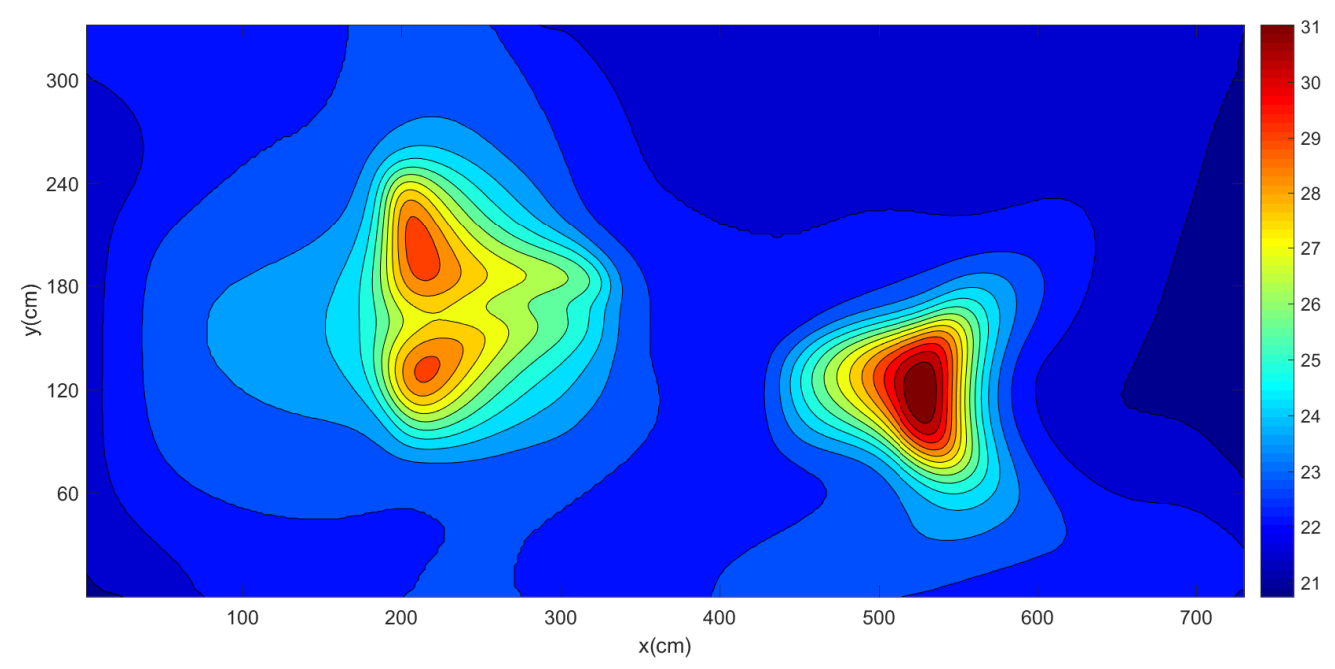

Figure 3.6: The ground truth $\left({ }^{\circ} \mathrm{C}\right)$ : the thermal map $1.2 \mathrm{~m}$ above the ground of the thermal lab in Figure 2.2, which is obtained from the CFD simulation.

\section{Thermal maps estimated from the observed input parameters}

Firstly, we estimate the thermal map from the observed input parameters. We use three methods (spline interpolation, standard ELM, and sELM) to train the 
regression model between the PCA coefficients and the input parameters. The estimated thermal maps are shown in Figures 3.7-3.9, receptively.

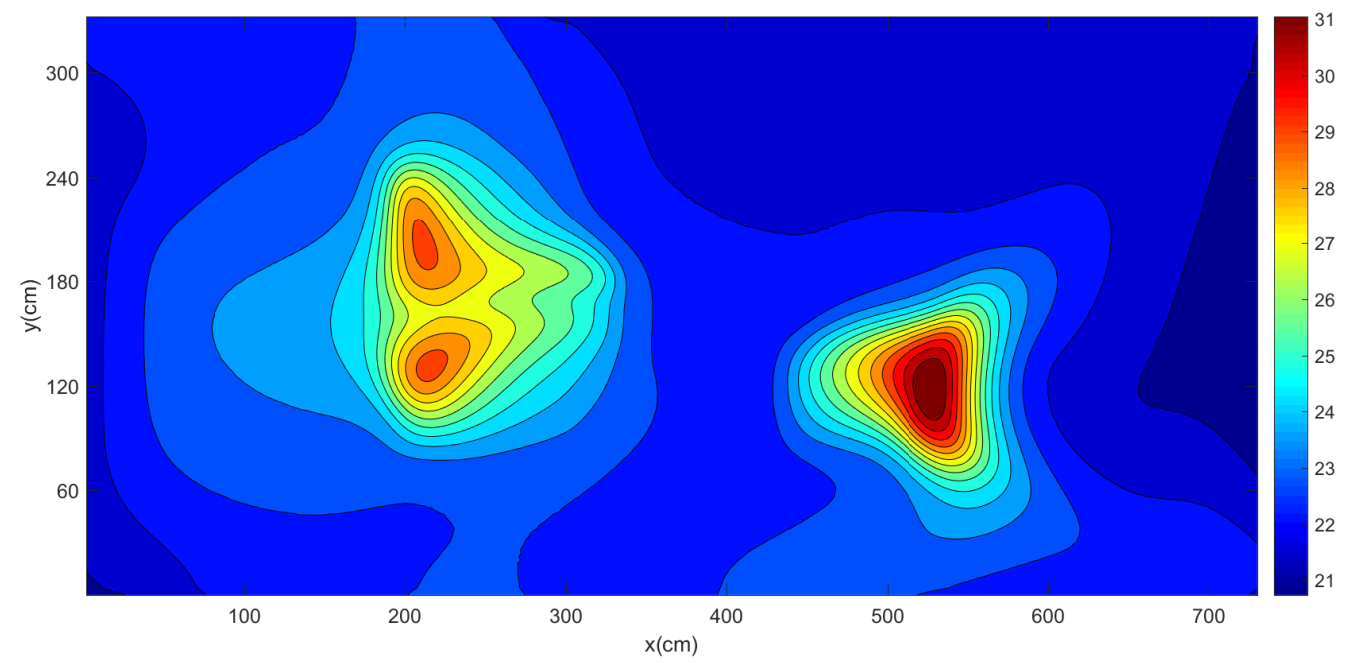

Figure 3.7: The estimated thermal map $\left({ }^{\circ} \mathrm{C}\right)$ : spline interpolation technique is used to estimate the PCA coefficients from the observed input parameters $\mathbf{p}_{\mathrm{o}}$.

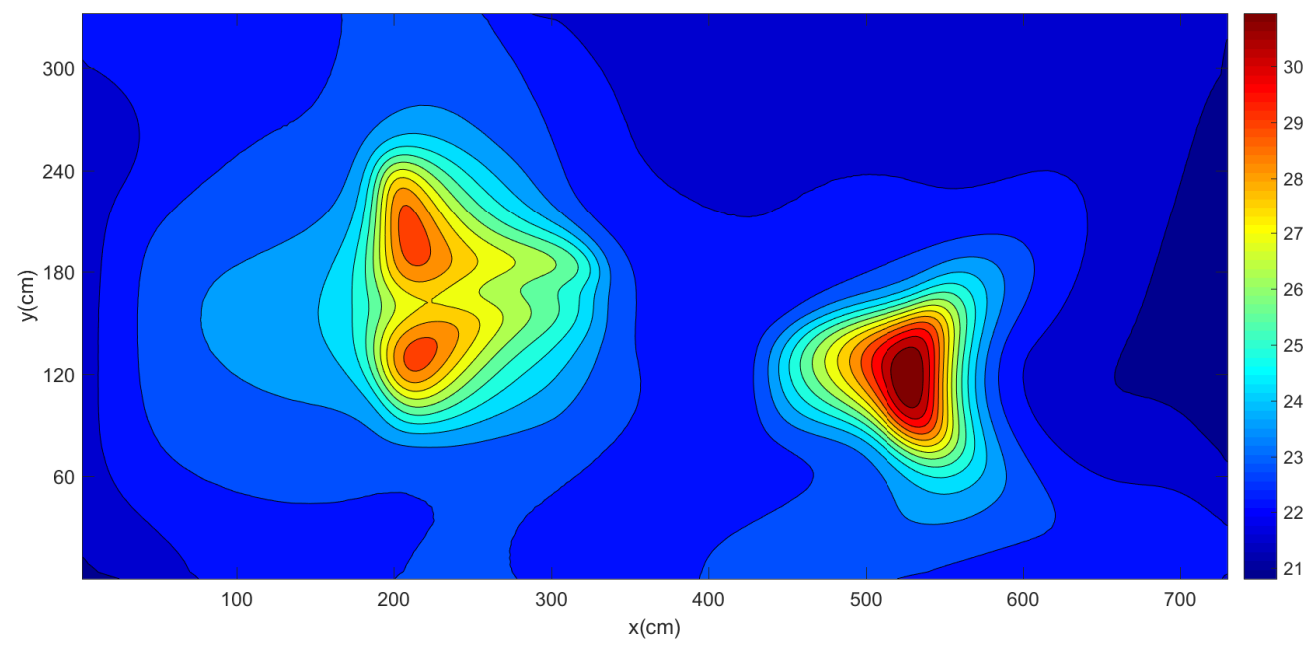

Figure 3.8: The estimated thermal map $\left({ }^{\circ} \mathrm{C}\right)$ : the standard ELM is used to estimate the PCA coefficients from the observed input parameters $\mathbf{p}_{\mathrm{o}}$. Both the random weights and the random bias follow the normal distribution $\mathcal{N}\left(0,0.00025^{2}\right)$. The number of hidden neurons $L=100$, and the regularization parameter $\gamma=0.00001$. 


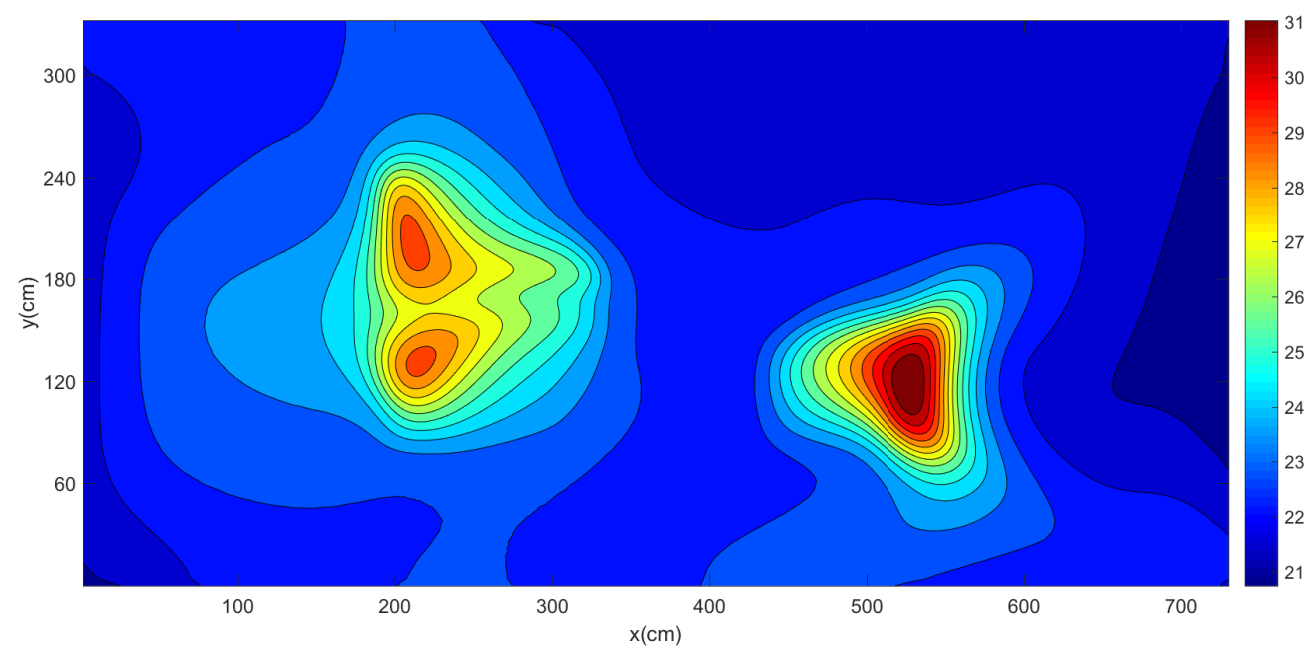

Figure 3.9: The estimated thermal map $\left({ }^{\circ} \mathrm{C}\right)$ : the sELM is used to estimate the PCA coefficients from the observed input parameters $\mathbf{p}_{\mathrm{o}}$. Both the random weights and the random bias follow the normal distribution $\mathcal{N}\left(0,0.025^{2}\right)$. The number of hidden neurons $L=100$, and the regularization parameter $\gamma=0.00001$.

\section{Thermal map estimated from the sparse sensor observations}

Then, we estimate the thermal map based on the four sensor observations. We use the least square technique to estimate the dominant PCA coefficients of the thermal map from the four sensor observations. With the four dominant PCA coefficients we can easily reconstruct the thermal map, which is shown in Figure 3.10 .

\section{Thermal maps estimated via TSPFR}

Next, we use the four sensor observations to enhance the thermal maps estimated from regression models which are trained by spline interpolation and sELM techniques, respectively. We firstly estimate the errors of the thermal maps in Figure 3.7 and Figure 3.9 from the four sensor observations. The method to estimate the error fields is the same as the method to estimate the thermal map from sparse sensor observations. The sensing locations are also the same as the stars shown in Fig. 3.10. 


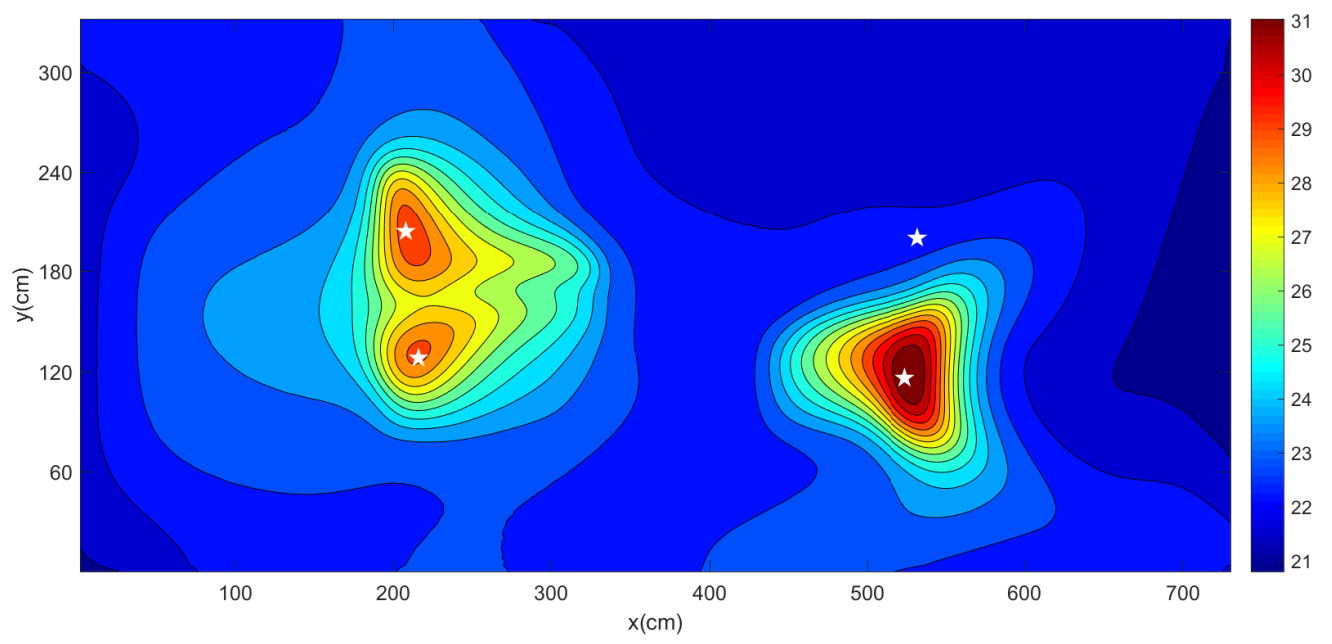

Figure 3.10: The estimated thermal map $\left({ }^{\circ} \mathrm{C}\right)$ : the least square technique is used to estimate the dominant PCA coefficients from the four sensor observations. The stars represent the sensing locations.

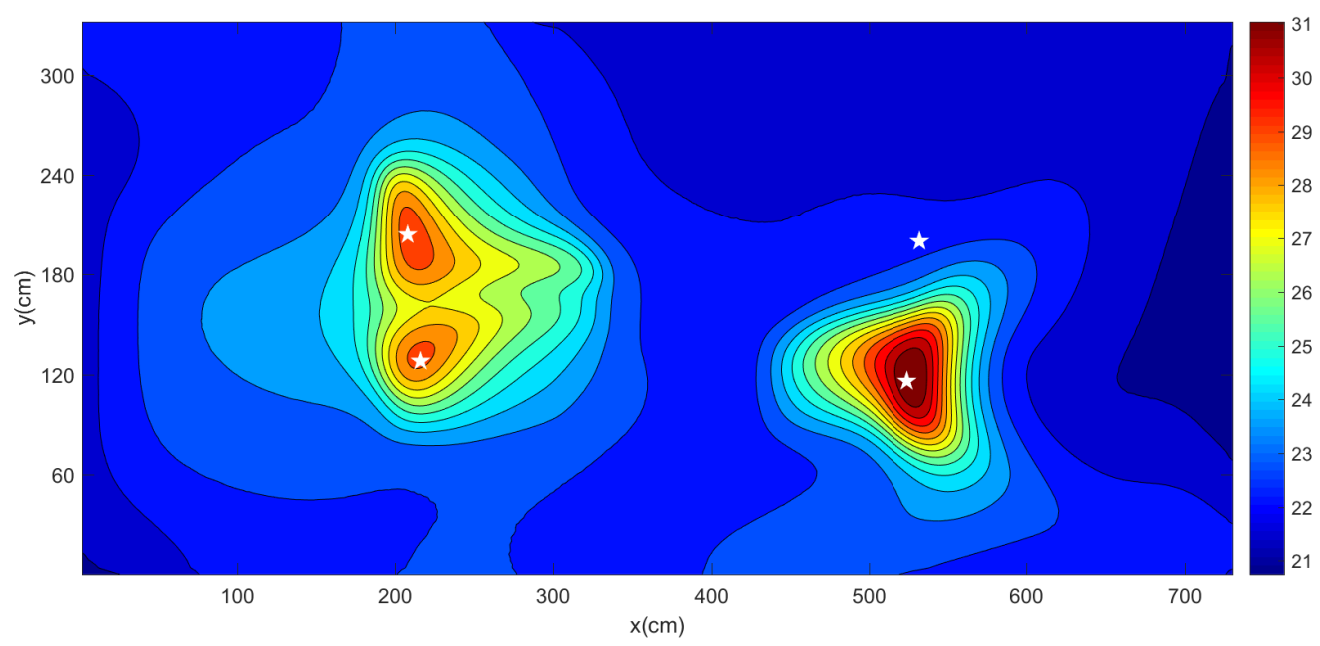

Figure 3.11: The estimated thermal map $\left({ }^{\circ} \mathrm{C}\right)$ : the TSPFR method is used to estimate the PCA coefficients from both the observed input parameters $\mathbf{p}_{\mathrm{o}}$, and the four sensor observations. In the first stage, we use the spline interpolation method to determine the regression model. The stars represent the sensing locations.

With the estimated error fields, we can easily correct the two thermal maps, respectively. Using the proposed TSPFR method, we can obtain the enhanced thermal maps, which are shown in Figure 3.11-3.12. 


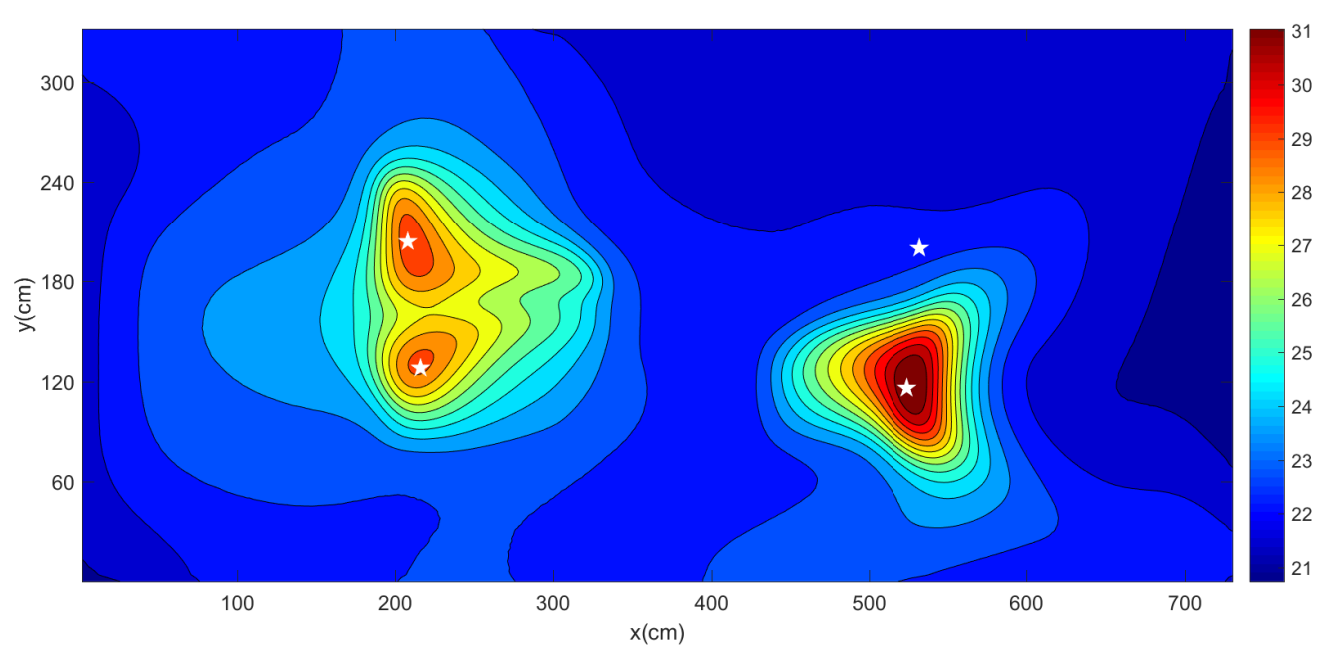

Figure 3.12: The estimated thermal map $\left({ }^{\circ} \mathrm{C}\right)$ : the TSPFR method is used to estimate the PCA coefficients from both the observed input parameters $\mathbf{p}_{\mathrm{o}}$, and the four sensor observations. In the first stage, we use sELM to train the regression model. The stars represent the sensing locations.

\section{The error fields of the estimated thermal maps}

Figures 3.7-3.12 look similar with the ground truth (i.e., the Figure 3.6), and we cannot directly evaluate the accuracy of the estimated thermal maps in Figures 3.7-3.12. Next, as shown in Figures 3.13-3.15, we provide error fields of the six estimated thermal maps.

\section{The performance indices}

To assess the performance of the estimated thermal maps, we introduce the following three performance indices:

1. Root mean square error:

$$
\operatorname{RMSE}(E)=\sqrt{\frac{1}{60955} \sum_{i=1}^{167} \sum_{j=1}^{365} e_{i j}^{2}}
$$




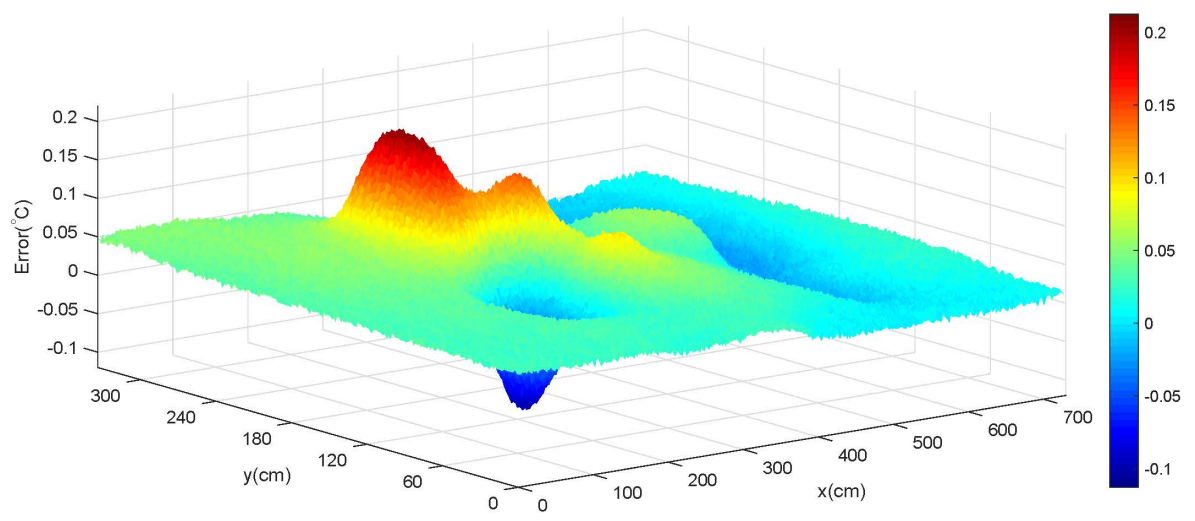

(A) The error of the estimated thermal map shown in Figure $3.7\left({ }^{\circ} \mathrm{C}\right)$. The regression model is trained by the spline interpolation technique.

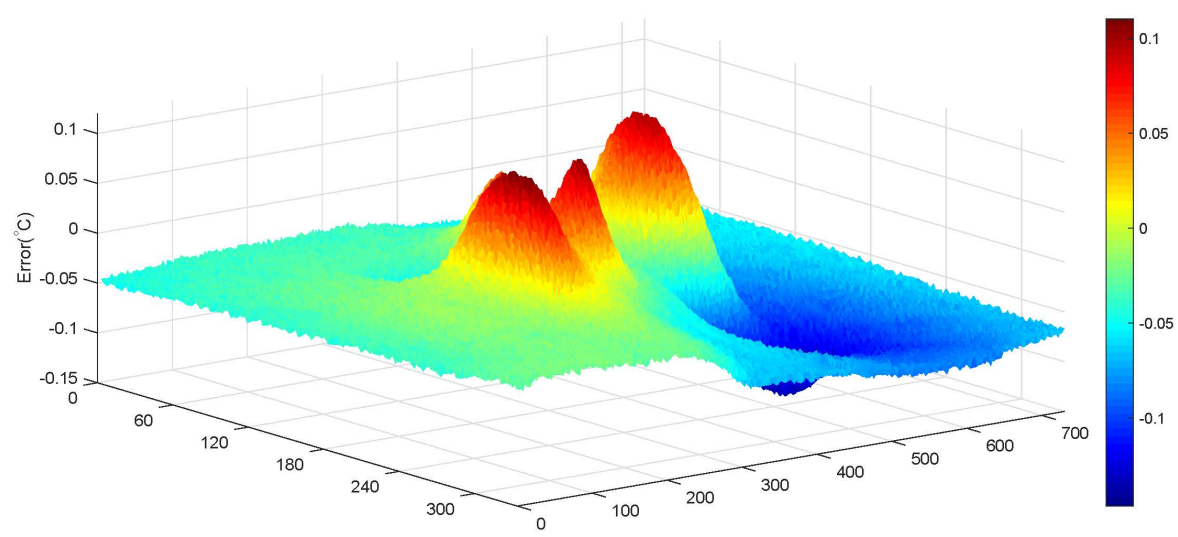

(в) The error of the estimated thermal map shown in Figure $3.8\left({ }^{\circ} \mathrm{C}\right)$. The regression model is trained by the standard ELM.

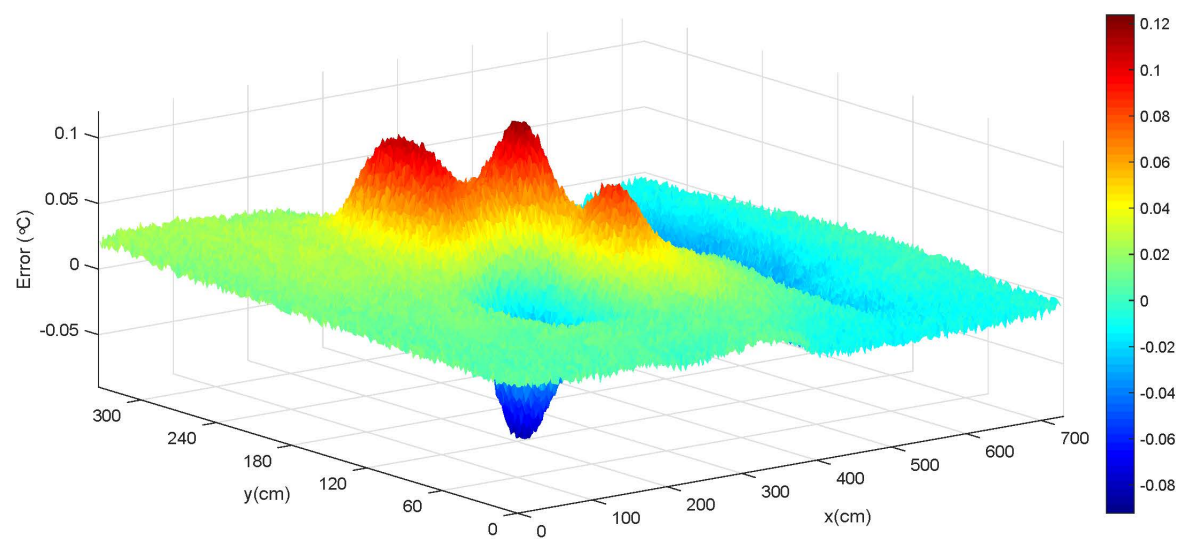

(C) The error of the estimated thermal map shown in Figure $3.9\left({ }^{\circ} \mathrm{C}\right)$. The regression model is trained by the sELM.

Figure 3.13: The error of estimated thermal maps. The PCA coefficients are estimated from the observed input parameters $\mathbf{p}_{\mathrm{o}}$ using three different regression models. 


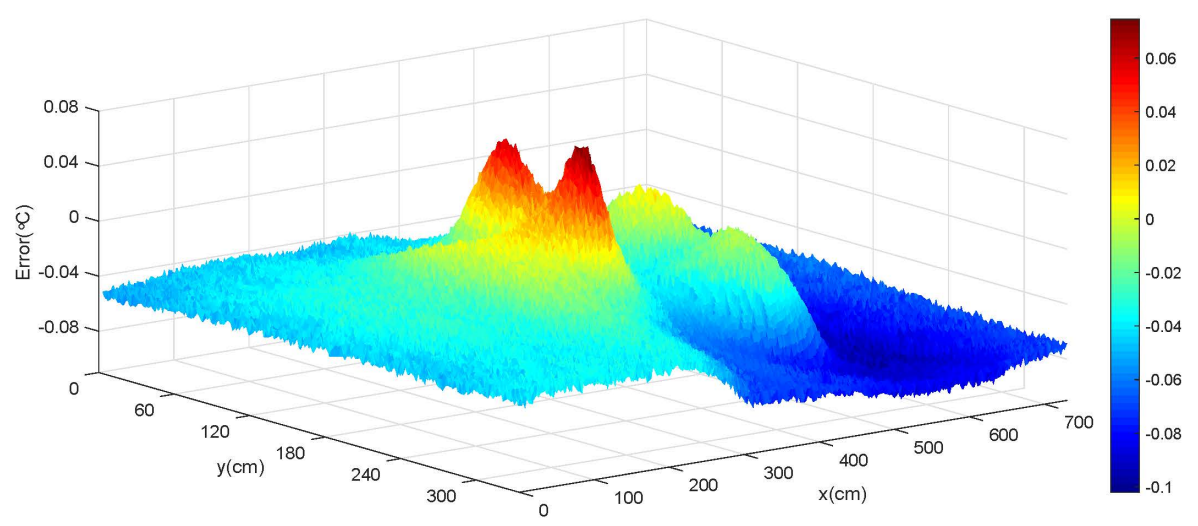

Figure 3.14: The error of the estimated thermal map in Figure $3.10\left({ }^{\circ} \mathrm{C}\right)$ : the least square technique is used to estimate the dominant PCA coefficients from the four sensor observations.

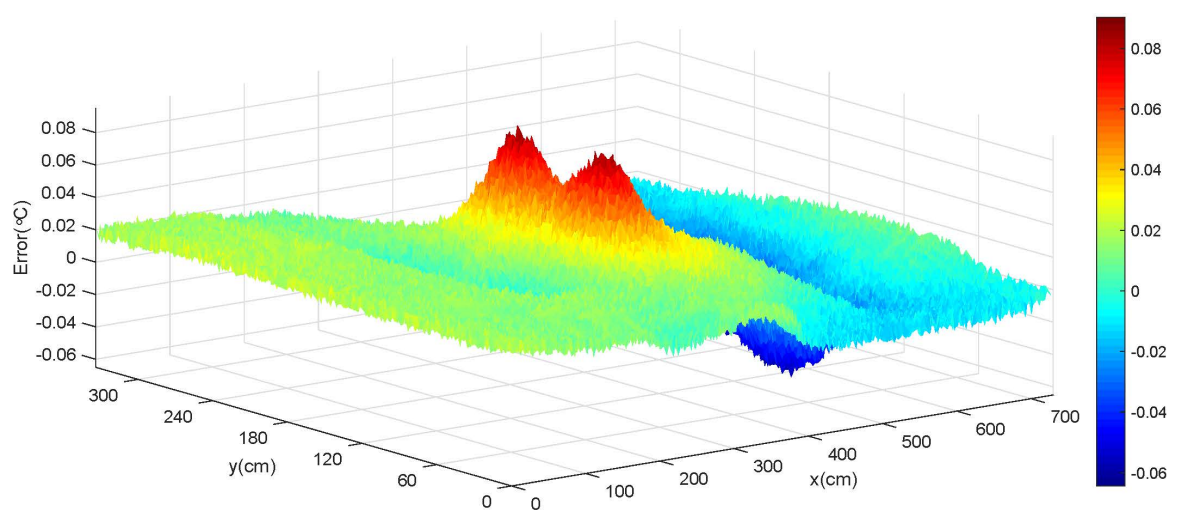

(A) The error of the estimated thermal map in Figure $3.11\left({ }^{\circ} \mathrm{C}\right)$. Spline interpolation technique is used to train the regression model in the first stage.

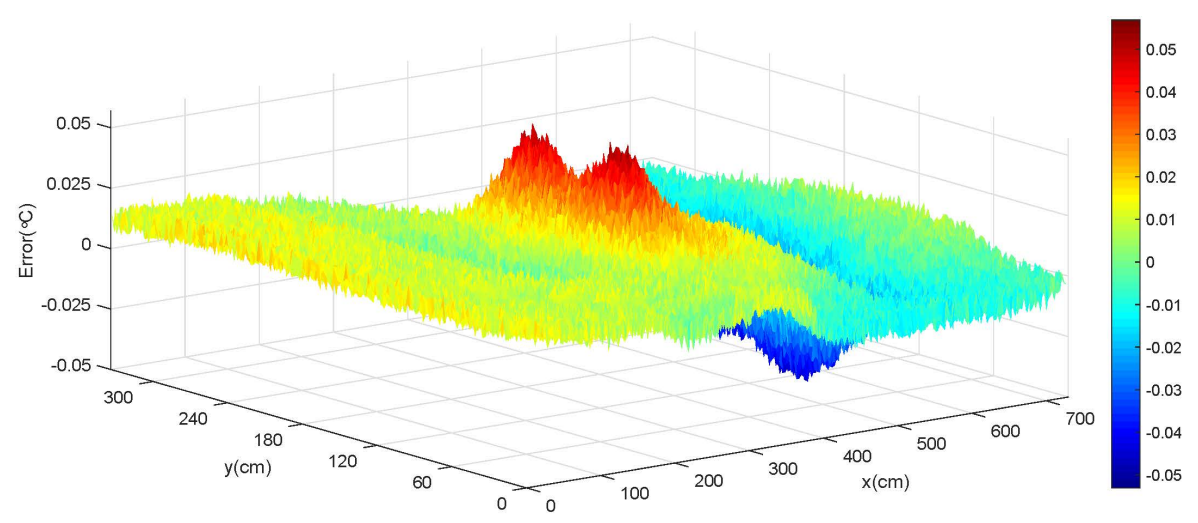

(B) The error of the estimated thermal map in Figure $3.12\left({ }^{\circ} \mathrm{C}\right)$. sELM is used to train the regression model in the first stage.

FiguRE 3.15: The error of the estimated thermal maps. The TSPFR method is used to estimate the PCA coefficients from both the observed input parameters $\mathbf{p}_{\mathrm{o}}$ and the four sensor observations. 
2. Sum of absolute error:

$$
\operatorname{SAE}(E)=\sum_{i=1}^{167} \sum_{j=1}^{365}\left|e_{i j}\right|
$$

3. Maximum absolute error:

$$
\operatorname{MAE}(E)=\max \left\{\left|e_{i j}\right|, 1 \leq i \leq 167,1 \leq j \leq 365\right\}
$$

where $E \in \mathbb{R}^{167 \times 365}$ is the error map of the estimated thermal map, and $e_{i j}$ is the entry of $E$ in the $i$-th row and the $j$-th column.

TABLE 3.2: The performance indices of the estimated thermal maps

\begin{tabular}{cccc}
\hline \hline & RMSE & SAE & MAE \\
\hline spline interpolation & 0.0430 & 1991.8 & 0.2112 \\
ELM & 0.0577 & 2808.2 & 0.1432 \\
sELM & 0.0332 & 1423.2 & 0.1221 \\
least square & 0.0521 & 2542.3 & 0.0999 \\
TSPFR-SI & 0.0224 & 1067.9 & 0.0902 \\
TSPFR-sELM & 0.0160 & 771.6 & 0.0569 \\
\hline \hline
\end{tabular}

Note: least square is the method to estimate the dominant PCA coefficients from four sensor observations. TSPFR-SI is the TSPFR method and spline interpolation technique is used to train the regression model in the first stage. TSPFR-sELM implies that sELM is used to train the regression model in the first stage. Four sensor observations are used for TSPFR-SI and TSPFR-sELM.

The performance indices of the six estimated thermal maps are given in Table 3.2. The RMSE and SAE reflect the overall performance of the estimated thermal maps. We can see from Table 3.2 that the overall performance of the sELM outperforms those of the spline interpolation and the standard ELM. It is clear that the proposed TSPFR method outperforms all the one stage methods in terms of the overall performance. If the proposed sELM is used to train the regression model in the first stage, the TSPFR method can provide the best overall performance.

The MAE reflects the local performance of the estimated thermal maps. Again the proposed TSPFR method has the best local performance. 
Remark 8. When using the standard ELM, tuning the model parameters is very important. For example, the standard deviation of the random weights. If the model parameters are not well-tuned, the estimation results are probably very bad. The sELM yields great improvement over the standard ELM, and the performance is more robust in terms of the variation of the model parameters.

Remark 9. When estimating the dominant PCA coefficients from sparse sensor observations, the sensing locations are very important. If the sensing locations are not well selected, the solution may be totally meaningless. For the TSPFR approach, if the sensing locations are not well selected, the second stage may yield a worse thermal map estimation.

TABLE 3.3: The mean absolute error (mAE) of the estimated PCA coefficients for leave-one out cross validation (LOOCV)

\begin{tabular}{ccc}
\hline \hline$i$-th PCA mode $(i)$ & spline interpolation & sELM \\
\hline 1 & 6.756 & 6.365 \\
2 & 1.714 & 1.557 \\
3 & 1.289 & 1.481 \\
4 & 12.391 & 11.972 \\
5 & 1.032 & 1.121 \\
6 & 0.862 & 0.735 \\
7 & 0.478 & 0.513 \\
\hline \hline
\end{tabular}

\subsubsection{Number reduction of the sensor observations}

Next, we consider the case that no enough observations of the physical field are available. Firstly, we test the performance of the regression models trained by spline interpolation and sELM using the LOOCV technique, respectively. The mean absolute error (mAE) of the estimated PCA coefficients are shown in Table 3.3.

It is clearly shown in Table 3.3 that for both the regression models trained by spline interpolation and sELM, the mAE of the first and fourth estimated PCA 


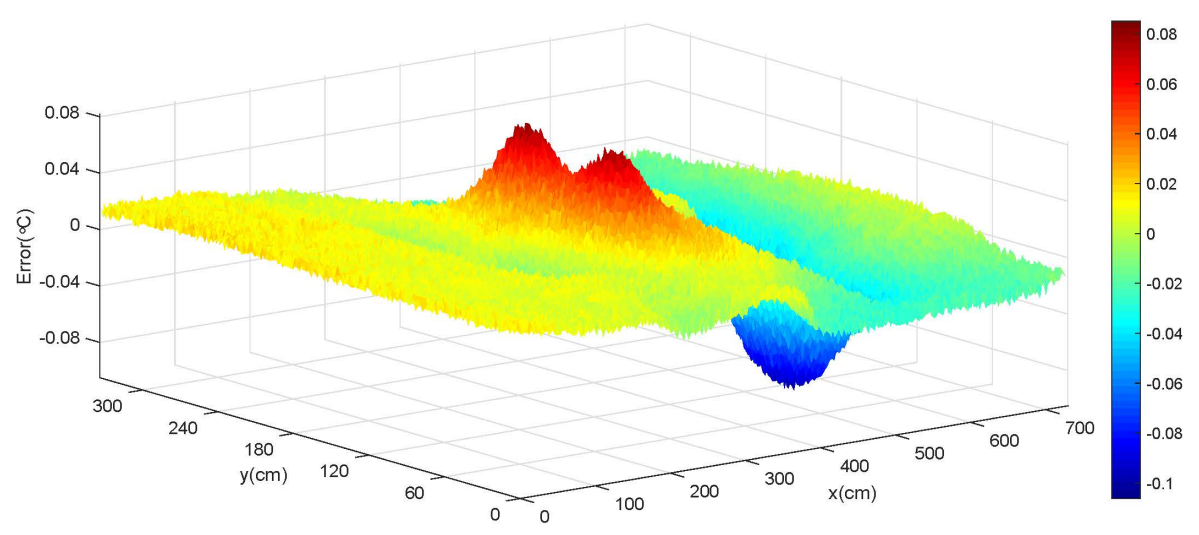

(A) The error field $\left({ }^{\circ} \mathrm{C}\right)$. Spline interpolation technique is used to train the regression model in the first stage.

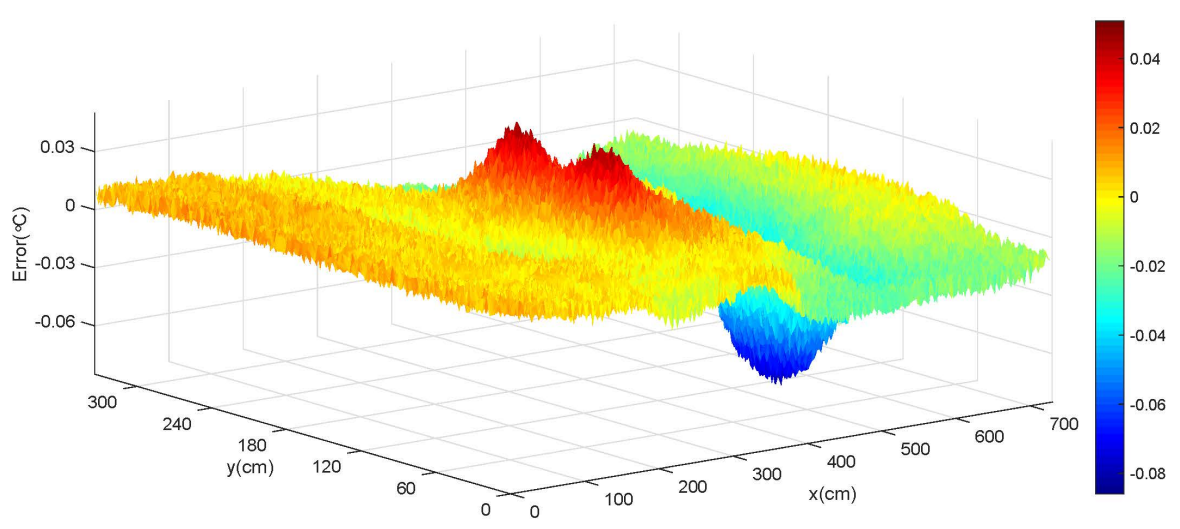

(B) The error field $\left({ }^{\circ} \mathrm{C}\right)$. sELM is used to train the regression model in the first stage.

FiguRE 3.16: The error of two estimated thermal maps. The TSPFR method is used to estimate the thermal map from the observed input parameters $\mathbf{p}_{\mathrm{o}}$ and TWO sensor observations. Only the first and the fourth PCA modes are used in the second stage.

coefficients are much larger than the others. Therefore, we can set the index set in Equation (3.48) as

$$
\mathcal{R}=\{1,4\}
$$

and $\tilde{\boldsymbol{\Phi}}_{\mathrm{r}}=\left[\boldsymbol{\varphi}_{1}, \boldsymbol{\varphi}_{4}\right]$.

Using the proposed TSPFR method with two sensor observations, we estimate the thermal map. In the first stage, we use spline interpolation and sELM to train the regression model, respectively. The error fields of the estimated thermal maps are shown in Figure 3.16. The performance indices of the two estimated thermal maps are shown in Table 3.4 . 
TABLE 3.4: The performance indices of the estimated thermal maps when the number of sensor observations is smaller than the number of dominant PCA modes.

\begin{tabular}{c|c|cccc}
\hline \hline NO. of sensors & $\mathcal{R}$ & method & RMSE & SAE & MAE \\
\hline \multirow{2}{*}{2} & \multirow{2}{*}{1,4} & TSPFR-SI & 0.0262 & 1243.4 & 0.1062 \\
& & TSPFR-sELM & 0.0211 & 997.3 & 0.0859 \\
\hline \multirow{2}{*}{3} & \multirow{2}{*}{$1,2,4$} & TSPFR-SI & 0.0244 & 1158.2 & 0.0948 \\
& \multirow{2}{*}{3} & TSPFR-sELM & 0.0179 & 869.4 & 0.0743 \\
\hline \multirow{2}{*}{$1,2,3$} & TSPFR-SI & 0.0391 & 1821.5 & 0.2738 \\
& & TSPFR-sELM & 0.0345 & 1617.3 & 0.2945 \\
\hline \hline
\end{tabular}

Note: $\mathcal{R}$ includes the indices that the PCA modes used in the second stage. TSPFR-SI: the TSPFR method and using spline interpolation technique in the first stage. TSPFR-sELM: the TSPFR method and using sELM in the first stage.

TABLE 3.5: The error of the dominant PCA coefficients estimated from different methods.

\begin{tabular}{|c|c|c|c|c|c|c|}
\hline NO. of sensors & $\mathcal{R}$ & method & mode1 & mode2 & mode3 & mode4 \\
\hline- & - & spline interp. & 6.6496 & 3.4088 & 1.4760 & 5.6940 \\
\hline- & - & sELM & 3.5517 & 2.6147 & 1.0087 & 6.2598 \\
\hline \multirow{2}{*}{4} & \multirow{2}{*}{$1,2,3,4$} & TSPFR-SI & 0.6702 & 2.8120 & -0.3965 & -0.2007 \\
\hline & & TSPFR-sELM & 0.0399 & 1.9773 & -0.2624 & -0.1547 \\
\hline \multirow{2}{*}{2} & \multirow{2}{*}{1,4} & TSPFR-SI & -1.2958 & 3.4088 & 1.4760 & -0.3590 \\
\hline & & TSPFR-sELM & -1.7948 & 2.6147 & 1.0087 & -0.2725 \\
\hline \multirow{2}{*}{3} & \multirow{2}{*}{$1,2,4$} & TSPFR-SI & 0.2184 & 3.1058 & 1.4760 & 0.6421 \\
\hline & & TSPFR-sELM & -0.0579 & 2.4277 & 1.0087 & -0.1893 \\
\hline \multirow{2}{*}{3} & \multirow{2}{*}{$1,2,3$} & TSPFR-SI & -6.3421 & -2.2787 & 0.3890 & 5.6940 \\
\hline & & TSPFR-sELM & -4.7232 & -2.4231 & 0.6301 & 6.2598 \\
\hline
\end{tabular}

Note: $\mathcal{R}$ includes the indices that the PCA modes used in the second stage. spline interp. \& sELM: the PCA coefficients are estimated from observed input parameters. spline interpolation and sELM are the methods to train the regression models. TSPFR-SI: the TSPFR method and using spline interpolation technique in the first stage. TSPFR-sELM: the TSPFR method and using sELM in the first stage.

Comparing with Figure 3.13 and Table 3.2, we can find from Figure 3.16 and Table 3.4 that the proposed TSPFR method can greatly improve the thermal maps 
estimated from the first stage with only two sensor observations. The proposed TSPFR method with two sensor observations also outperforms the one stage method with four sensor observations for the physical field reconstruction.

If three sensor observations are available, the performance indices of the estimated thermal maps are also shown in Table 3.4. Base on the results of LOOCV, we should use PCA modes $(1,2$, and 4$)$ in the second stage. If we set $\mathcal{R}=\{1,2,4\}$, as shown in Table 3.4, all the performance indices have a small improvement. However, if we do not consider the results of LOOCV and simply set $\mathcal{R}=\{1,2,3\}$, i.e., using the first three dominant PCA modes in the second stage, as shown in Table 3.4, the estimation results would become worse and even worse than those of the first stage results. To analyze the reason, we compute the error of the estimated PCA coefficients, which is shown in Table 3.5

From Table 3.5, we can find that for any PCA mode that is not used in the second stage, the estimated PCA component has no change, i.e, the error of the PCA coefficient will be the same as that of the fist stage result.

The errors of the dominant PCA coefficient when we set $\mathcal{R}=\{1,2,3\}$ are larger than those when we set $\mathcal{R}=\{1,2,4\}$. As analyzed in Section 3.4.3 - 3.4.4, if we ignore influence of sensing locations and measurement noise, the error energy of the proposed TSPFR method mainly depends on the truncation error. Table 3.5 show that the error of the fourth PCA component estimated in the first stage is quite large. If we set $\mathcal{R}=\{1,2,3\}$, the error of the fourth PCA component estimated in the first stage will be a part of the truncation error. Hence, we cannot obtain the accurate results.

In practice, however, we can previously determine the optimal set of PCA modes used in the second stage based on the number of available sensors and the results of LOOCV for the regression model used in the first stage. 


\subsection{Concluding remarks}

With known PCA modes, we can reconstruct the physical field of interest if the PCA coefficients are estimated. The PCA coefficients can be estimated from the observed input parameters via a regression model. We present a SLFN (regression model) trained by sELM to estimate all the involved PCA coefficients. The proposed regression model is shown to be better and more convenient than the commonly used models obtained from spline interpolation. When using the spline interpolation, we need to train many regression models, while using the neural network, only one multi-input-multi-output regression model requires to be trained.

The dominant PCA coefficients can also be estimated from sparse sensor observations. In this method, only the dominant PCA coefficients are estimated and the error of the reconstructed field is positively correlated with the energy of the dropped subdominant PCA components (i.e., the truncation error). In the proposed twostage physical field reconstruction method, we combine the two approaches. We estimate all the involved PCA coefficients from the regression model in the first stage, and estimate the error of the physical field estimated in the first stage from sparse sensor observations. The truncation error of the error field is smaller than that of the physical field itself; therefore, the error of the estimated error field will be smaller than the error of the physical field directly estimated from sparse sensor observations. Consequently, with the estimated error field we can enhance the physical field estimated in the first stage.

If we directly estimate one physical field from sparse senor observations, the number of sensor observations must be not less than the number of dominant PCA modes of the physical field. However, if we use the proposed TSPFR method, we can reduce the number of required sensors. Some of the PCA components of the physical field estimated in the first stage are enough accurate, which is not required to be enhanced. In the second stage, we can ignore the corresponding PCA components of the error field. The number of PCA modes used in the second stage is reduced. Thus, the number of sensor observations can be reduced. In practice, we can use 
the cross validation technique to test the performance of the regression model used in the frits stage and hance to find the PCA components which are well estimated in the first stage. 


\section{Chapter 4}

\section{Sensor placement for the optimal physical field reconstruction}

As mentioned in Chapter 3, sensing locations play a critical role in the reconstruction of a physical field. In this chapter, we provide a novel greedy algorithm for the sensor placement problem. This chapter is organized as follows. In Section 4.1, we introduce the problem of physical field reconstruction and the sensor placement problem. In Section 4.2, we briefly review three current methods for the sensor placement problem. In Section 4.3, we develop two new algorithms: the minimum nonzero eigenvalue pursuit (MNEP) algorithm and the maximal projection on minimum eigenspace (MPME) algorithm. We then provide four examples to compare the effectiveness of MPME with the current methods via Monte-Carlo simulations in Section 4.4. In Section 4.5, we analyze the computational cost of the MPME algorithm and compare it with those of current methods. In Section 4.6, we apply the proposed MPME algorithm for the thermal map reconstruction (the example in Chapter 3), and show that the state of the arts cannot work due to the high storage requirement. The conclusions are given in Section 4.7. 


\subsection{Problem formulation}

In this section, we firstly review the physical field reconstruction from sparse sensor observations. Then, we formulate the problem of sensor placement for the optimal physical field reconstruction to be a cardinality optimization problem. For the two stage physical field reconstruction (TSPFR) method proposed in Chapter 3, in the second stage, we can solve the cardinality optimization problem to determine the optimal sensing locations.

\subsubsection{Physical field reconstruction}

We consider a physical field $\mathbf{f} \in \mathbb{R}^{N}$ described as

$$
\mathbf{f}=\tilde{\Phi} \alpha
$$

where $\boldsymbol{\alpha} \in \mathbb{R}^{n}$ is a vector of parameters to be estimated with $n \ll N$, and $\tilde{\boldsymbol{\Phi}} \in \mathbb{R}^{N \times n}$ is a known full column-rank matrix, which we call the signal representation matrix and its column vectors compose a basis of the physical field. For the physical field reconstruction problem in Chapter 3, the signal representation matrix actually consists of the dominant PCA coefficients, and $\boldsymbol{\alpha}$ is the dominant PCA coefficients.

It is expensive and impractical to sense the physical field $\mathbf{f}$ with $N$ sensor nodes since $N$ is very large and depends on the resolution of the discrete physical space [65]. However, part of the physical field can be observed from sensor networks, i.e.

$$
\mathrm{y}=\mathrm{Hf}+\boldsymbol{\nu}=\mathbf{\Phi} \alpha+\nu
$$

where $\mathbf{H} \in \mathbb{R}^{M \times N}$ whose $i$-th row is $\mathbf{e}_{s_{i}}^{\mathrm{T}}, s_{i} \in \mathcal{N}=\{1,2, \ldots, N\}$ corresponds to the $i$-th sensing location, and $M(n \leq M \ll N)$ is the number of sensor nodes. The observation matrix

$$
\boldsymbol{\Phi}=\mathbf{H} \tilde{\boldsymbol{\Phi}}=\left[\boldsymbol{\varphi}_{s_{1}}, \boldsymbol{\varphi}_{s_{2}}, \ldots \boldsymbol{\varphi}_{s_{M}}\right]^{\mathrm{T}}
$$


is a pruned matrix from the rows of $\tilde{\boldsymbol{\Phi}}$ indexed by $\left\{s_{1}, s_{2}, \ldots, s_{M}\right\}$, and $\boldsymbol{\varphi}_{s_{i}}^{\mathrm{T}}$ is the $s_{i}$-th row of $\tilde{\boldsymbol{\Phi}}$ and represents the observation model of the $i$-th sensor node, which we call the observation vector. The measurement noise $\boldsymbol{\nu} \in \mathbb{R}^{M}$ is assumed to be zero-mean i.i.d. Gaussian random process with variance $\sigma^{2} \mathbf{I}$.

From (4.2), we can obtain the following minimum variance unbiased estimate (MVUE)

$$
\hat{\boldsymbol{\alpha}}=\Phi^{\dagger} \mathbf{y}
$$

where $\boldsymbol{\Phi}^{\dagger}=\left(\boldsymbol{\Phi}^{\mathrm{T}} \boldsymbol{\Phi}\right)^{-1} \boldsymbol{\Phi}^{\mathrm{T}}$ is the pseudo-inverse of $\boldsymbol{\Phi}$. The mean square error (MSE) of this MVUE $[64,65,69]$ is

$$
\operatorname{MSE}(\hat{\boldsymbol{\alpha}})=\mathbb{E}\left(\|\hat{\boldsymbol{\alpha}}-\boldsymbol{\alpha}\|_{2}^{2}\right)=\sigma^{2} \operatorname{tr}\left(\Psi^{-1}\right)=\sigma^{2} \sum_{k=1}^{n} \frac{1}{\lambda_{k}}
$$

where $\lambda_{1} \geq \lambda_{2} \geq \ldots \geq \lambda_{n}$ stand for the eigenvalues of

$$
\Psi=\Phi^{\mathrm{T}} \boldsymbol{\Phi}
$$

which we call the dual observation matrix.

With some standard operations, we can obtain the variance of $\hat{\boldsymbol{\alpha}}$ as

$$
\begin{aligned}
\boldsymbol{\Sigma} & =\mathbb{E}\left[(\hat{\boldsymbol{\alpha}}-\boldsymbol{\alpha})(\hat{\boldsymbol{\alpha}}-\boldsymbol{\alpha})^{\mathrm{T}}\right] \\
& =\mathbb{E}\left[\boldsymbol{\Phi}^{\dagger} \boldsymbol{\nu} \boldsymbol{\nu}^{\mathrm{T}}\left(\boldsymbol{\Phi}^{\dagger}\right)^{\mathrm{T}}\right] \\
& =\sigma^{2} \boldsymbol{\Phi}^{\dagger}\left(\boldsymbol{\Phi}^{\dagger}\right)^{\mathrm{T}}=\sigma^{2} \boldsymbol{\Psi}^{-1}
\end{aligned}
$$

Then, we introduce the following worst case error variance (WCEV) of the MVUE $\hat{\alpha}$

$$
\operatorname{WCEV}(\hat{\boldsymbol{\alpha}})=\max _{\|\mathbf{x}\|_{2}=1} \mathbf{x}^{\mathrm{T}} \boldsymbol{\Sigma} \mathbf{x}=\lambda_{\max }(\boldsymbol{\Sigma})=\frac{\sigma^{2}}{\lambda_{n}}
$$

For more detail about WCEV, do refer to [64]. $\operatorname{Both} \operatorname{MSE}(\hat{\boldsymbol{\alpha}})$ and $\operatorname{WCEV}(\hat{\boldsymbol{\alpha}})$ are indicators to access the performance of the MVUE $\hat{\boldsymbol{\alpha}}$. Since

$$
\boldsymbol{\Phi}^{\dagger}\left(\boldsymbol{\Phi}^{\dagger}\right)^{\mathrm{T}}=\boldsymbol{\Psi}^{-1}
$$


it is easily found from (4.4) and (4.5) that

$$
\begin{aligned}
\operatorname{MSE}(\hat{\boldsymbol{\alpha}}) & =\sigma^{2}\left\|\boldsymbol{\Phi}^{\dagger}\right\|_{\mathrm{F}}^{2}=\sigma^{2} \sum_{k=1}^{n} \frac{1}{\lambda_{k}} \\
\operatorname{WCEV}(\hat{\boldsymbol{\alpha}}) & =\sigma^{2}\left\|\boldsymbol{\Phi}^{\dagger}\right\|_{2}^{2}=\frac{\sigma^{2}}{\lambda_{n}}
\end{aligned}
$$

Consequently, the two error indicators are equivalent due to the equivalence of the two matrix norms [83]. Specifically,

$$
\operatorname{WCEV}(\hat{\boldsymbol{\alpha}}) \leq \operatorname{MSE}(\hat{\boldsymbol{\alpha}}) \leq n \operatorname{WCEV}(\hat{\boldsymbol{\alpha}})
$$

It is clear in (4.4) and (4.5) that both MSE and WCEV depend on the eigenvalues of the dual observation matrix $\boldsymbol{\Psi}$, which fully depends on the sensing locations.

\subsubsection{Sensor placement}

We denote the set of selected sensing locations by $\mathcal{S}=\left\{s_{1}, s_{2}, \ldots, s_{M}\right\}$, and the set of potential sensing locations by $\mathcal{N}=\{1,2, \ldots, N\}$, which correspond to the row indices of $\boldsymbol{\Phi}$ and $\tilde{\boldsymbol{\Phi}}$, respectively. Then, we formulate the following sensor placement problem.

Problem 2. Given the signal representation matrix $\tilde{\boldsymbol{\Phi}}=\left[\boldsymbol{\varphi}_{1}, \boldsymbol{\varphi}_{2}, \ldots, \boldsymbol{\varphi}_{N}\right]^{\mathrm{T}} \in \mathbb{R}^{N \times n}$, select $M$ rows of $\tilde{\boldsymbol{\Phi}}$ indexed by $s_{1}, s_{2}, \ldots, s_{M}$ to construct the observation matrix $\boldsymbol{\Phi}=\left[\boldsymbol{\varphi}_{s_{1}}, \boldsymbol{\varphi}_{s_{2}}, \ldots, \boldsymbol{\varphi}_{s_{M}}\right]^{\mathrm{T}} \in \mathbb{R}^{M \times n}$, such that the error of the estimated parameters $\hat{\boldsymbol{\alpha}}$ in (4.3) is small enough and the number of rows of $\boldsymbol{\Phi}$, i.e. $M$, is minimized.

This actually is a sensing location selection problem. We aim to find the minimum number of sensing locations with which the error of $\hat{\boldsymbol{\alpha}}$ is less than a predefined threshold. In this chapter, we use the WCEV as the error indicator, and Equation (4.6) shows that a small WCEV can guarantee a small MSE. Then, this sensor placement problem can be formulated as the following cardinality minimization 
problem

$$
\hat{\mathcal{S}}=\underset{\mathcal{S} \subseteq \mathcal{N}}{\arg \min }|\mathcal{S}| \quad \text { subject to } \quad \lambda_{n} \geq \gamma
$$

where $|\cdot|$ returns the cardinality of a set, and $\gamma$ corresponds to the maximum acceptable WCEV.

\subsection{The state of the art}

The combinatorial optimization problem (4.7) is NP-hard [99]. Here, we briefly review three current and related methods. Two of them are originally designed for the case where the number of available sensors is fixed. However, they can be simply modified and applied to the case where the number of sensors is unknown, which is discussed in Remark 10.

\subsubsection{Convex relaxation}

This method was detailed in [64]. If the number of sensor nodes is fixed, the sensor placement problem can be formulated as

$$
\begin{array}{cl}
\text { maximize } & \log \operatorname{det}\left(\sum_{i=1}^{N} w_{i} \boldsymbol{\varphi}_{i} \boldsymbol{\varphi}_{i}^{\mathrm{T}}\right) \\
\text { subject to } & \mathbf{1}^{\mathrm{T}} \mathbf{w}=M \\
& w_{i} \in\{0,1\}, \quad i \in \mathcal{N}
\end{array}
$$

with variable $\mathbf{w} \in \mathbb{R}^{N}$. Here $w_{i}=1$ means $i \in \mathcal{S}$ and $w_{i}=0$ means $i \notin \mathcal{S}$. Performing a convex relaxation, i.e. replacing the nonconvex Boolean constraints $w_{i} \in\{0,1\}$ by $w_{i} \in[0,1]$, we can obtain the following convex optimization problem:

$$
\begin{array}{ll}
\text { maximize } & \log \operatorname{det}\left(\sum_{i=1}^{N} w_{i} \boldsymbol{\varphi}_{i} \boldsymbol{\varphi}_{i}^{\mathrm{T}}\right) \\
\text { subject to } & \mathbf{1}^{\mathrm{T}} \mathbf{w}=M \\
& w_{i} \in[0,1], \quad i \in \mathcal{N}
\end{array}
$$


with variable $\mathbf{w}$. This problem can be solved by the interior-point methods [100]. Rearranging the entries of the solution of the relaxed problem (4.9), i.e. $\mathbf{w}^{*}(\in$ $\left.[0,1]^{N}\right)$, in descending order yields the sequence $\left\{w_{\hat{s}_{1}}^{*}, w_{\hat{s}_{2}}^{*}, \ldots, w_{\hat{s}_{N}}^{*}\right\}$. Then, the set of the sensing indices is given by $\hat{\mathcal{S}}=\left\{\hat{s}_{1}, \hat{s}_{2}, \ldots, \hat{s}_{M}\right\}$, i.e. the indices of the $M$ largest elements of $\mathbf{w}^{*}$.

\subsubsection{SparSenSe}

This method was detailed in [69]. To determine the number of required sensor nodes, the following convex optimization, called sparse-aware sensor selection (SparSenSe), is formulated:

$$
\begin{array}{cl}
\operatorname{minimize} & \|\mathbf{w}\|_{l 1} \\
\text { subject to } & {\left[\begin{array}{cr}
\sum_{i=1}^{N} w_{i} \boldsymbol{\varphi}_{i} \boldsymbol{\varphi}_{i}^{\mathrm{T}} & \mathbf{e}_{j} \\
\mathbf{e}_{j}^{\mathrm{T}} & x_{j}
\end{array}\right] \succeq \mathbf{0}, j=1, \ldots, n} \\
& \|\mathbf{x}\|_{l 1} \leq \gamma^{\prime}, x_{j} \geq 0, j=1, \ldots, n \\
& w_{i} \in[0,1], \quad i \in \mathcal{N}
\end{array}
$$

where $\gamma^{\prime}$ corresponds to the maximum acceptable MSE index. This is a linear matrix inequalities problem and can be solved by using the CVX toolbox [101]. With the solution $\mathbf{w}^{*}$ and a prior threshold $\tau(0 \lesssim \tau \ll 1)$, we can determine the sensing indices. If $w_{i}^{*}<\tau$, set $w_{i}^{*}=0$. The number of required sensor nodes is the number of nonzero entries of $\mathbf{w}^{*}$.

\subsubsection{FrameSense}

This method was detailed in [65]. The ensemble of the rows of a matrix can be viewed as a frame. If all the observation vectors have the same norm, according to the frame theory, the observation matrix $\boldsymbol{\Phi}$ achieves the minimum MSE when it achieves the minimum frame potential [65, 102]. For the basic concept of the 
frame theory, do refer to [103]. The sensor placement problem can be solved by minimizing the following frame potential

$$
\operatorname{FP}(\boldsymbol{\Phi})=\sum_{i, j \in \mathcal{S}}\left(\boldsymbol{\varphi}_{i}^{\mathrm{T}} \boldsymbol{\varphi}_{j}\right)^{2}
$$

One greedy "worst-out" algorithm, called the FrameSense, can provide a nearoptimal solution in the sense of the minimum frame potential. At each step, it removes the row of $\tilde{\boldsymbol{\Phi}}$ that maximally increases the frame potential. If $\tilde{\boldsymbol{\Phi}}$ corresponds to an equal-norm frame, the row index is in fact the index of the row/column

of $\left(\tilde{\boldsymbol{\Phi}} \tilde{\boldsymbol{\Phi}}^{\mathrm{T}}\right)^{2}$ which has the largest 1-norm. Here, $\mathbf{A}^{2}$ denotes a matrix whose entries are the square of the corresponding entries of $\mathbf{A}$.

Remark 10. With simple modifications, convex relaxation and FrameSense can be used to determine the least number of required sensors.

- For the convex relaxation method, it can be found by increasing the sensor number from $n$ until the constraint in (4.7) is satisfied.

- For FrameSense, when removing each row of $\tilde{\boldsymbol{\Phi}}$, we check the constraint in (4.7). If the constraint is not satisfied, reserve the row and the number of remaining rows of $\tilde{\boldsymbol{\Phi}}$ is the least number of required sensors.

Besides the sensor placement for physical field reconstruction, many other excellent sensor placement works have focused on the continuous system [70], nonlinear model [76], energy saving [66, 78], state estimation for dynamic system [78, 79, 104107], and Gaussian process interpolation [108-111].

\subsection{The proposed algorithms}

As mentioned before, one apparent method to solve the cardinality optimization problem (4.7) is to evaluate the minimum eigenvalue of the dual observation matrix 
(i.e. $\left.\quad \lambda_{n}\right)$ of all potential sensor configurations, and then find the configuration with the minimum number of sensors that satisfies the constraint. Unfortunately, the computational cost of exhaustively searching $2^{N}$ potential configurations is unaffordable for large scale problems. One simple strategy to reduce the number of searched sensor configurations is to determine the sensing locations one-by-one. With such a strategy, the minimum number of required sensor nodes, $M$, can be easily found by judging whether the constraint in (4.7) is satisfied after each sensing location is determined. In this way, the number of searched sensor configurations can be reduced to $\sum_{i=0}^{M-1}(N-i)$, since at the first step over $N$ possible sensing locations are searched, then $N-1$, and so on.

Admittedly, each sensor node may have correlated influence with others, and one sensor reading is informative for a given sensor configuration but may be meaningless for others. When finding the sensing locations one-by-one, we do not know the contribution of each sensor node for the final sensor configuration; therefore, such a strategy cannot guarantee the optimal solution. However, we are trying to make a tradeoff between the computational cost and the number of required sensor nodes, i.e. to find an effective sensor configuration with proper number of sensor nodes by determining the sensing locations one-by-one.

When determining the sensing locations one-by-one, we can obtain an observation vector sequence $\left\{\boldsymbol{\varphi}_{s_{k}}\right\}_{k=1}^{M}$. For simplicity, we introduce a new matrix $\boldsymbol{\Phi}_{k} \in \mathbb{R}^{k \times n}$ to denote the first $k(1 \leq k \leq M)$ rows of $\boldsymbol{\Phi}$, i.e. $\boldsymbol{\Phi}_{k}=\left[\boldsymbol{\varphi}_{s_{1}}, \boldsymbol{\varphi}_{s_{2}}, \ldots \boldsymbol{\varphi}_{s_{k}}\right]^{\mathrm{T}}$ corresponds to the first $k$ sensing locations. Corresponding to $\left\{\boldsymbol{\varphi}_{s_{k}}\right\}_{k=1}^{M}$, we can obtain the matrix sequence $\left\{\boldsymbol{\Phi}_{k}\right\}_{k=1}^{M}$ and the dual observation matrix sequence $\left\{\boldsymbol{\Psi}_{k}\right\}_{k=1}^{M}$. Here $\boldsymbol{\Psi}_{k}=\boldsymbol{\Phi}_{k}^{\mathrm{T}} \boldsymbol{\Phi}_{k}$ has a nonincreasing eigenvalue sequence $\left\{\lambda_{i}^{(k)}\right\}_{i=1}^{n}$. The notations that will commonly appear in this chapter are listed below for easy reference.

For all $k<n$, the minimum eigenvalue of $\boldsymbol{\Psi}_{k}, \lambda_{n}^{(k)}=0$ because $\operatorname{rank}\left(\boldsymbol{\Psi}_{k}\right)=$ $\operatorname{rank}\left(\Phi_{k}\right) \leq k$. Therefore, the number of required sensor nodes $M$ must be no less than $n$. Since $\tilde{\boldsymbol{\Phi}}$ is a full column-rank matrix, the nonsingular $\boldsymbol{\Psi}_{n}$ exists, which implies that the optimal $M$ may be equal to $n$. Consequently, to minimize $M$ we 


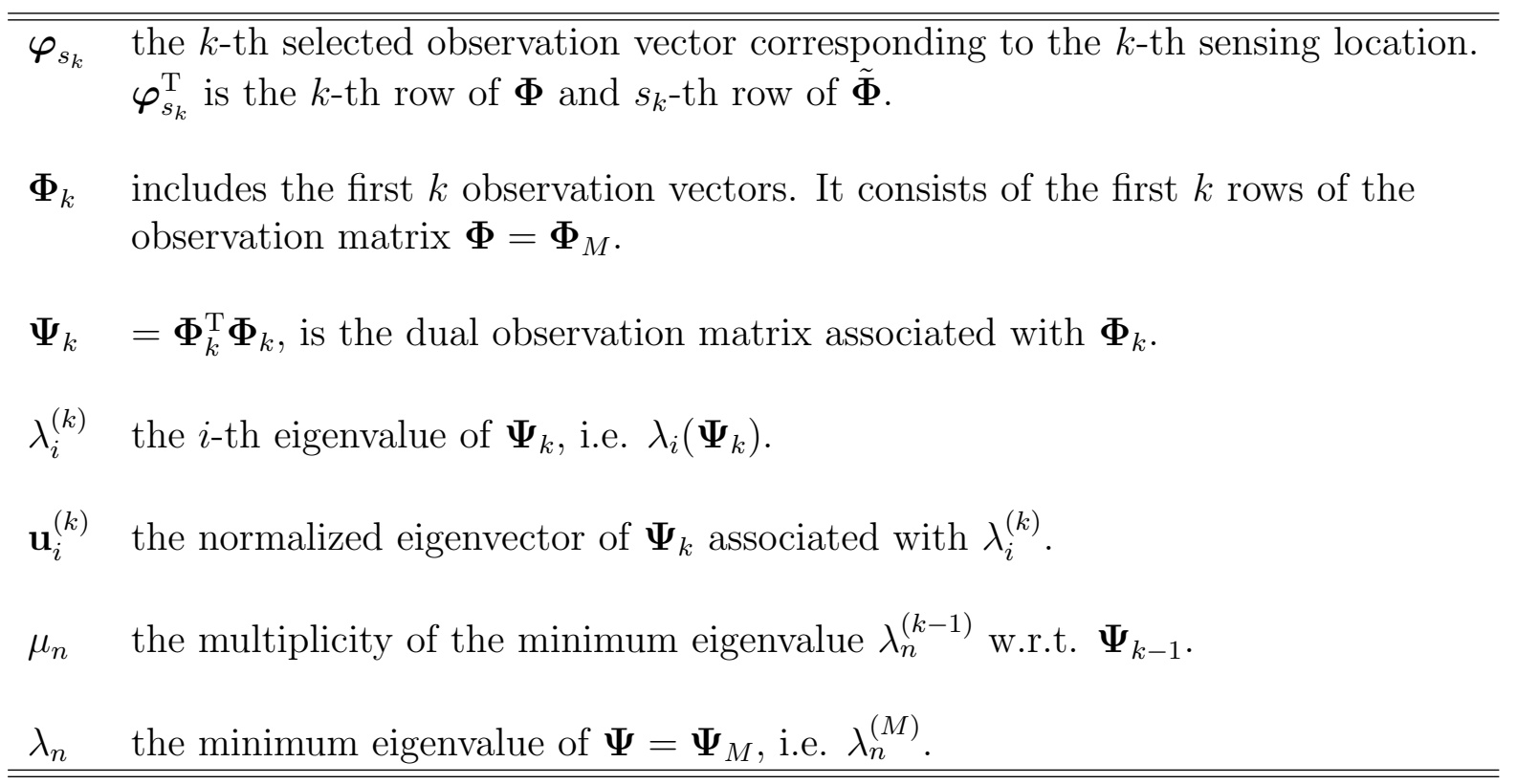

should guarantee that $\boldsymbol{\Psi}_{n}$ is nonsingular. In that case, the vectors in $\left\{\boldsymbol{\varphi}_{s_{k}}\right\}_{k=1}^{n}$ are mutually independent and

$$
\operatorname{rank}\left(\boldsymbol{\Psi}_{k}\right)=\operatorname{rank}\left(\boldsymbol{\Phi}_{k}\right)=\operatorname{rank}\left(\left\{\boldsymbol{\varphi}_{s_{i}}\right\}_{i=1}^{k}\right)=k
$$

for all $k \leq n$. In practice, we can easily guarantee that $\boldsymbol{\varphi}_{s_{k}}$ is independent with the vectors in $\left\{\boldsymbol{\varphi}_{s_{i}}\right\}_{i=1}^{k-1}$ when determining the $k$-th sensing location for all $k \leq n$.

Our purpose is to find the shortest observation vector sequence $\left\{\boldsymbol{\varphi}_{s_{k}}\right\}_{k=1}^{M}$ by determining the sensing locations one-by-one, such that the constraint in (4.7), i.e. $\lambda_{n} \geq \gamma$, is satisfied. For a given sensor configuration corresponding to $\boldsymbol{\Phi}_{k-1}(1 \leq$ $k<M)$, we need to formulate some guidelines to determine the next sensing location $s_{k}$. Intuitively, we can traverse all $N-k+1$ unselected observation vectors to find the one that can maximally increase $\lambda_{n}$. Then, the other sensing locations can be similarly determined one-by-one until $\lambda_{n}>\gamma$ and meanwhile the number of selected sensing locations is the minimum number of required sensor nodes, i.e. $M$.

However, the challenge is that we do not know how $\varphi_{s_{k}}$ affects $\lambda_{n}\left(=\lambda_{n}^{(M)}\right)$ since $M$ and $\left\{\boldsymbol{\varphi}_{s_{i}}\right\}_{i=k+1}^{M}$ are unknown when we find the $k$-th sensing location. In other 
words, we cannot build an explicit mapping between $\varphi_{s_{k}}$ and $\lambda_{n}$. Therefore, it is impossible to find the optimal $k$-th sensing location $s_{k}^{*}$ by directly optimizing $\lambda_{n}$.

Our main idea is to find a new criterion instead of $\lambda_{n}$. When determining the $k$-th sensing location, we optimize the new criterion. The criterion should satisfy three conditions:

1. It can be directly obtained from $\boldsymbol{\Phi}_{k-1}$ and $\boldsymbol{\varphi}_{s_{k}}$.

2. Optimizing the criterion can guarantee that all the vectors in $\left\{\boldsymbol{\varphi}_{s_{k}}\right\}_{k=1}^{n}$ are mutually independent.

3. $\lambda_{n}$ is positively correlated with the criterion.

The first condition implies that we can directly assess the contributions of all potential observation vectors for the new criterion. The second condition guarantees that $\boldsymbol{\Psi}_{n}$ is nonsingular, and the last condition guarantees that increasing the new criterion can increase $\lambda_{n}$, i.e. decrease the WCEV. If such a criterion exists, we can determine the sensing locations one-by-one by maximizing the new criterion. Accordingly, we can find a suboptimal sensor configuration. In what follows, we shall present two alternative criteria.

\subsubsection{Minimum nonzero eigenvalue pursuit (MNEP)}

If we determine the sensing locations one-by-one, corresponding to the observation vector sequence $\left\{\boldsymbol{\varphi}_{s_{k}}\right\}_{k=1}^{M}$, we can obtain the dual observation matrix sequence $\left\{\boldsymbol{\Psi}_{k}\right\}_{k=1}^{M}$. It can be easily found that

$$
\boldsymbol{\Psi}_{k}=\boldsymbol{\Phi}_{k}^{\mathrm{T}} \boldsymbol{\Phi}_{k}=\left[\begin{array}{ll}
\boldsymbol{\Phi}_{k-1}^{\mathrm{T}} & \boldsymbol{\varphi}_{s_{k}}
\end{array}\right]\left[\begin{array}{ll}
\boldsymbol{\Phi}_{k-1}^{\mathrm{T}} & \boldsymbol{\varphi}_{s_{k}}
\end{array}\right]^{\mathrm{T}}=\boldsymbol{\Psi}_{k-1}+\boldsymbol{\varphi}_{s_{k}} \boldsymbol{\varphi}_{s_{k}}^{\mathrm{T}}
$$

This equation implies that all the eigenvalues of $\boldsymbol{\Psi}_{k}$ satisfy the first condition of the criterion used to replace $\lambda_{n}$, i.e. the eigenvalues of $\boldsymbol{\Psi}_{k}$ can be found if $\boldsymbol{\Phi}_{k-1}$ and $\boldsymbol{\varphi}_{s_{k}}$ are known. Amongst all the eigenvalues of $\boldsymbol{\Psi}_{k}$, we guess that the minimum 
nonzero eigenvalue, i.e. $\lambda_{k}^{(k)}$ when $k \leq n$ and $\lambda_{n}^{(k)}$ when $k>n$, is one choice of the criterion to be used to replace $\lambda_{n}$.

Obviously, for any $k \leq n$, maximizing $\lambda_{k}^{(k)}$ can guarantee that $\lambda_{k}^{(k)}>0$, which implies that for any $k \leq n$, the vectors in $\left\{\boldsymbol{\varphi}_{s_{i}}\right\}_{i=1}^{k}$ are mutually independent. Therefore, the minimum nonzero eigenvalue of $\boldsymbol{\Psi}_{k}$ satisfies the second condition. We then utilize the following theorem to show that it also satisfies the third condition.

Theorem 1. Suppose $\mathbf{B}=\mathbf{A}+\mathbf{c c}^{\mathrm{T}}$ where $\mathbf{A} \in \mathbb{R}^{n \times n}$ is symmetric, and $\mathbf{c} \in \mathbb{R}^{n}$ is a non-zero vector. Then,

$$
\lambda_{1}(\mathbf{B}) \geq \lambda_{1}(\mathbf{A}) \geq \lambda_{2}(\mathbf{B}) \geq \lambda_{2}(\mathbf{A}) \geq \ldots \geq \lambda_{n}(\mathbf{B}) \geq \lambda_{n}(\mathbf{A})
$$

Proof. See [112] and the Theorem 8.1.8 in [83].

Since $\boldsymbol{\Psi}_{k}=\boldsymbol{\Psi}_{k-1}+\boldsymbol{\varphi}_{s_{k}} \boldsymbol{\varphi}_{s_{k}}^{\mathrm{T}}$, considering Theorem 1, we can obtain

$$
\begin{array}{ll}
\lambda_{n}^{(k)} \geq \lambda_{n}^{(k-1)} & \text { for all } \quad k \geq n \\
\lambda_{k}^{(k)} \leq \lambda_{k-1}^{(k-1)} & \text { for all } \quad k \leq n
\end{array}
$$

From the two equations, we can easily find that

$$
\begin{aligned}
& \lambda_{n}^{(M)} \geq \lambda_{n}^{(M-1)} \geq \ldots \geq \lambda_{n}^{(n)} \\
& \lambda_{n}^{(n)} \leq \lambda_{n-1}^{(n-1)} \leq \ldots \leq \lambda_{1}^{(1)}
\end{aligned}
$$

For any $n \leq k<M$, (4.12) shows that $\lambda_{n}^{(k)}$ is the lower bound of $\lambda_{n}^{(k+1)}$. If we maximize $\lambda_{n}^{(k)}$ by proper selection of $\boldsymbol{\varphi}_{s_{k}}$, we maximize the lower bound of $\lambda_{n}^{(k+1)}$. Hence, $\lambda_{n}^{(k+1)}$ is positively correlated with $\lambda_{n}^{(k)}$. Since

$$
\lambda_{n}=\lambda_{n}^{(M)} \geq \lambda_{n}^{(M-1)} \geq \ldots \geq \lambda_{n}^{(k+1)} \geq \lambda_{n}^{(k)}
$$

$\lambda_{n}$ is positively correlated with $\lambda_{n}^{(k)}$ for any $n \leq k<M$. 
For any $k<n,(4.13)$ shows that $\lambda_{k}^{(k)}$ is the upper bound of $\lambda_{k+1}^{(k+1)}$. Therefore, if we select sensing location to maximize $\lambda_{k}^{(k)}$, we maximize the upper bound of $\lambda_{n}^{(n)}$. Actually, $\lambda_{k+1}^{(k+1)}$ is monotonically increasing w.r.t. $\lambda_{k}^{(k)}$ for any $k<n$, which will be shown later. Hence, $\lambda_{n}^{(n)}$ is monotonically increasing w.r.t. $\lambda_{k}^{(k)}$ for all $k<n$. Since $\lambda_{n}=\lambda_{n}^{(M)}$ is positively correlated with $\lambda_{n}^{(n)}$, we conclude that $\lambda_{n}$ is also positively correlated with $\lambda_{k}^{(k)}$ for any $k<n$.

In summary, $\lambda_{n}$ is positively correlated with the minimum nonzero eigenvalue of $\boldsymbol{\Psi}_{k}$, i.e. $\lambda_{k}^{(k)}$ for $k<n$ and $\lambda_{n}^{(k)}$ for $k \leq n$, which satisfies the third condition.

Therefore, the minimum nonzero eigenvalue of $\boldsymbol{\Psi}_{k}$ can be a criteria in place of $\lambda_{n}$ to optimize the $k$-th sensing location. To maximize $\lambda_{n}$, we can select $\boldsymbol{\varphi}_{s_{k}}$ to maximize $\lambda_{k}^{(k)}$ when $k<n$ and maximize $\lambda_{n}^{(k)}$ when $k \geq n$. The greedy algorithm for the sensor placement problem named minimum nonzero eigenvalue pursuit (MNEP) is summarized in Algorithm 1.

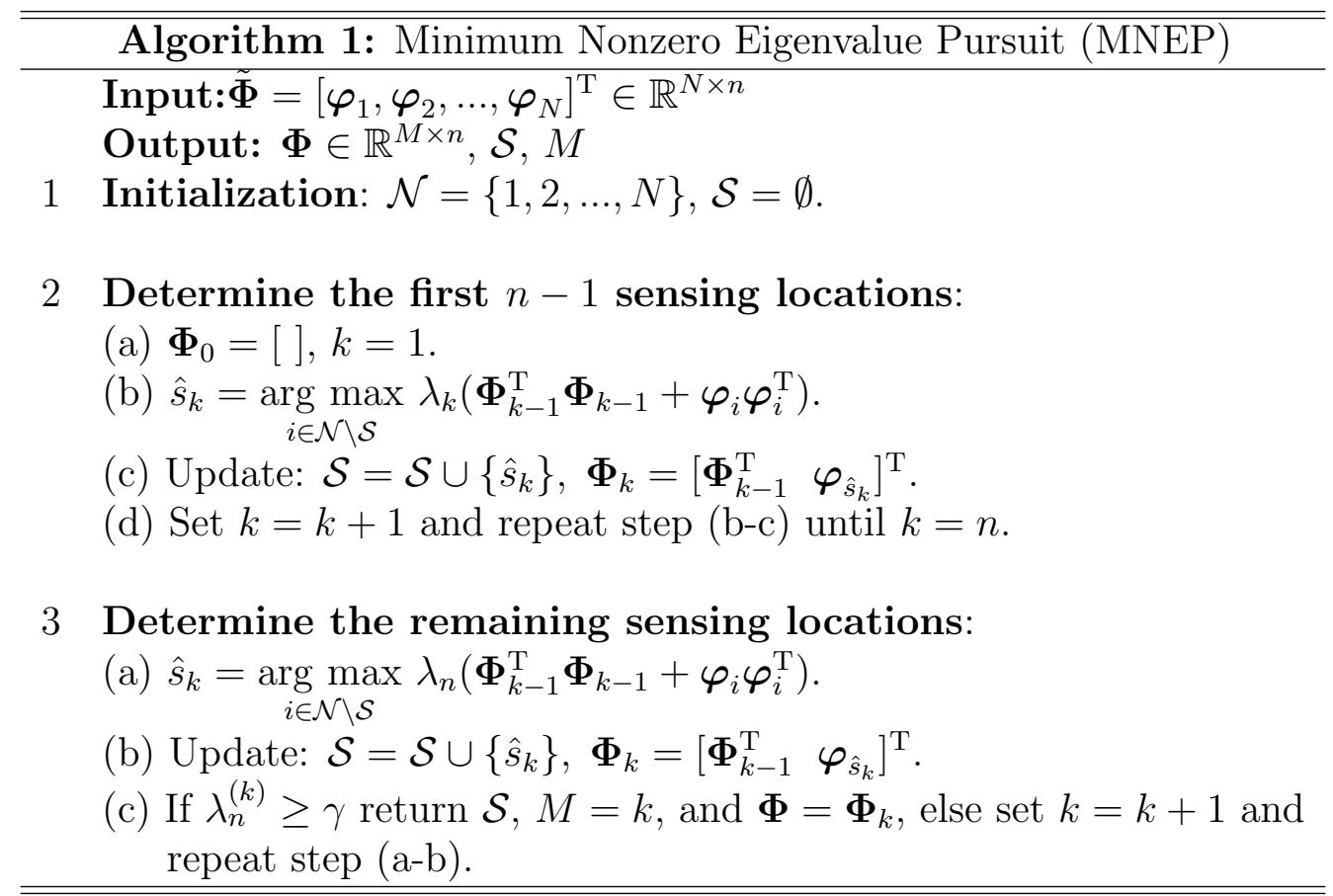

We determine the sensing locations one-by-one. For the first $n-1$ sensors, the $k$-th sensing location can be obtained from the optimization problem in step 2(b). To solve this optimization problem, we traverse all the unselected observation vectors 
and find the one that maximizes $\lambda_{k}^{(k)}$. After $n-1$ sensing locations have been determined, we find the remaining sensing locations by solving the optimization problem in step 3(a), which is similar to the previous one but maximizes $\lambda_{n}^{(k)}$, i.e. the minimum eigenvalue of $\boldsymbol{\Psi}_{k}$. Meanwhile, we check the constraint in (4.7) after each sensing location is determined. If the constraint is satisfied, stop the algorithm.

When determining the $k$-th sensing location, we need to traverse $N-k+1$ rows of $\tilde{\boldsymbol{\Phi}}$, and evaluate the minimum nonzero eigenvalue of $\boldsymbol{\Psi}_{k}$ for each case. Solving eigenvalue problems for all $N-k+1$ cases is computationally expensive. Can we find a simpler alternative criterion for the observation vectors in $\left\{\boldsymbol{\varphi}_{i}\right\}_{i=1}^{N}$ to avoid solving eigenvalue problems for all possible dual observation matrices?

In what follows, we provide another criterion, i.e. the magnitude of the projection of $\boldsymbol{\varphi}_{s_{k}}$ onto the minimum eigenspace of $\boldsymbol{\Psi}_{k-1}$, and it is shown to satisfy the three aforementioned conditions. Compared with the MNEP, the greedy algorithm via optimizing the new criterion is computationally more efficient and effective. The definition of minimum eigenspace will be given later.

\subsubsection{Maximal projection on minimum eigenspace (MPME)}

Sensor placement is about selecting proper observation vectors to guarantee that the eigenvalues of $\boldsymbol{\Psi}_{M}$ meet certain requirements. To understand how the observation vectors $\left\{\boldsymbol{\varphi}_{s_{i}}\right\}_{i=1}^{k}$ affect the eigenvalues of $\boldsymbol{\Psi}_{k}$, we introduce the following theorem.

Theorem 2. For any matrix $\boldsymbol{\Phi}_{k} \in \mathbb{R}^{k \times n}$, the symmetric matrix $\boldsymbol{\Psi}_{k}=\boldsymbol{\Phi}_{k}^{\mathrm{T}} \boldsymbol{\Phi}_{k}$ has a nonincreasing eigenvalue sequence $\left\{\lambda_{i}^{(k)}\right\}_{i=1}^{n}$, and

$$
\lambda_{i}^{(k)}=\left\|\boldsymbol{\Phi}_{k} \mathbf{u}_{i}^{(k)}\right\|_{2}^{2}=\sum_{j=1}^{k}\left(\boldsymbol{\varphi}_{s_{j}}^{\mathrm{T}} \mathbf{u}_{i}^{(k)}\right)^{2}
$$

where $\mathbf{u}_{i}^{(k)}$ is the normalized eigenvector associated with $\lambda_{i}^{(k)}$. 
Proof. See Appendix A.

In (4.14), $\boldsymbol{\varphi}_{s_{j}}^{\mathrm{T}} \mathbf{u}_{i}^{(k)}$ represents the magnitude of the projection of $\boldsymbol{\varphi}_{s_{j}}$ onto the eigenspace $\operatorname{span}\left(\mathbf{u}_{i}^{(k)}\right)$, which is associated with the eigenvalue $\lambda_{i}^{(k)}$. Theorem 2 shows that the eigenvalue of $\boldsymbol{\Psi}_{k}$ equals the square summation of the projections of all columns of $\boldsymbol{\Phi}_{k}^{\mathrm{T}}$ onto its eigenspace.

For any $k \geq n$, the minimum eigenvalue of $\boldsymbol{\Psi}_{k}$, i.e. $\lambda_{n}^{(k)}$, equals the square summation of the projections of $\left\{\boldsymbol{\varphi}_{s_{i}}\right\}_{i=1}^{k}$ onto the eigenspace associated with the minimum eigenvalue. However, before the $k$-th sensing location is determined, the eigenspace of $\boldsymbol{\Psi}_{k}$ is unknown. Therefore, to assess the contribution of the all unselected observation vectors for $\lambda_{n}^{(k)}$, we need to solve the eigenvalue problems for all potential cases, like the MNEP, which is computationally expensive.

To analyze the relation between the observation vectors in $\left\{\boldsymbol{\varphi}_{s_{i}}\right\}_{i=1}^{k}$ and the eigenspace associated with the minimum eigenvalue of $\boldsymbol{\Psi}_{k}$, we present the following theorem.

Theorem 3. The normalized eigenvector associated with $\lambda_{n}^{(k)}$ of $\boldsymbol{\Psi}_{k}$

$$
\mathbf{u}_{n}^{(k)}=\underset{\|\mathbf{x}\|_{2}=1}{\arg \min }\left\|\boldsymbol{\Phi}_{k} \mathbf{x}\right\|_{2}^{2}
$$

and one sufficient and necessary condition of $\lambda_{n}^{(k)}>\gamma$ is that for any nonzero normalized vector $\mathbf{x} \in \mathbb{R}^{n}$,

$$
\left\|\Phi_{k} \mathbf{x}\right\|_{2}^{2}>\gamma
$$

Proof. See Appendix B.

For any $k>n$, Equation (4.15) shows that $\operatorname{span}\left(\mathbf{u}_{n}^{(k-1)}\right)$ is the subspace onto which the square summation of the projections of $\left\{\boldsymbol{\varphi}_{s_{i}}\right\}_{i=1}^{k-1}$ is minimum. However, if the minimum eigenvalue of $\boldsymbol{\Psi}_{k-1}$, i.e. $\lambda_{n}^{(k-1)}$, is a multiple eigenvalue with multiplicity $\mu_{n}$, considering Equations (4.14) and (4.15) we can find that the optimization problem in (4.15) has $\mu_{n}$ different optimal solutions, which are exactly 
the normalized eigenvectors associated with the minimum eigenvalue of $\boldsymbol{\Psi}_{k-1}$, i.e. $\lambda_{n-\mu_{n}+1}^{(k-1)}=\lambda_{n-\mu_{n}+2}^{(k-1)}=\ldots=\lambda_{n}^{(k-1)}$.

For any $k \leq n$, the projection of any vector in $\left\{\boldsymbol{\varphi}_{s_{i}}\right\}_{i=1}^{k-1}$ onto the null space of $\boldsymbol{\Phi}_{k-1}$, i.e. $\operatorname{null}\left(\boldsymbol{\Phi}_{k-1}\right)$, is zero. It is clear that the square summation of the projections of $\left\{\boldsymbol{\varphi}_{s_{i}}\right\}_{i=1}^{k-1}$ onto any other subspace except the subspace of $\operatorname{null}\left(\boldsymbol{\Phi}_{k-1}\right)$ is nonzero. Therefore, $\operatorname{null}\left(\boldsymbol{\Phi}_{k-1}\right)$ is exactly the subspace with the highest dimension onto which the square summation of the projections of all vectors in $\left\{\boldsymbol{\varphi}_{s_{i}}\right\}_{i=1}^{k-1}$ is minimum.

For simplicity, we introduce a new concept of minimum eigenspace as follows:

Definition 1. For any positive semi-define symmetric matrix $\mathbf{A} \in \mathbb{R}^{n \times n}$ with the nonincreasing eigenvalue sequence $\left\{\lambda_{i}(\mathbf{A})\right\}_{i=1}^{n}$, the minimum eigenspace of $\mathbf{A}$ is the eigenspace associated with all the minimum eigenvalues of $\mathbf{A}$, i.e.

$$
\mathbb{U}_{k: n}(\mathbf{A})=\operatorname{span}\left(\mathbf{u}_{k}, \mathbf{u}_{k+1}, \ldots, \mathbf{u}_{n}\right)
$$

where $\mathbf{u}_{i}$ is the eigenvector associated with $\lambda_{i}(\mathbf{A})$, and $\lambda_{k-1}(\mathbf{A})>\lambda_{k}(\mathbf{A})=\lambda_{n}(\mathbf{A})$.

Equation (4.16) implies that to meet the requirement on $\lambda_{n}\left(=\lambda_{n}^{(M)}\right)$, i.e. $\lambda_{n} \geq \gamma$, the square summation of the projections of $\left\{\boldsymbol{\varphi}_{s_{k}}\right\}_{k=1}^{M}$ onto any non-trivial subspace of $\mathbb{R}^{n}$ should be larger than $\gamma$. Therefore, it is reasonable that when determining the $k$-th sensing location we select the observation vector $\boldsymbol{\varphi}_{s_{k}^{*}}$ that has the largest projection onto the non-trivial subspace onto which the square summation of the projections of $\left\{\boldsymbol{\varphi}_{s_{i}}\right\}_{i=1}^{k-1}$ is minimum.

Therefore, if $k \leq n$ we can select the $k$-th sensing location whose observation vector has the largest projection onto the null space of $\boldsymbol{\Phi}_{k-1}$. It is easily found that the null space of $\boldsymbol{\Phi}_{k-1}$ is exactly the minimum eigenspace of $\boldsymbol{\Psi}_{k-1}$, i.e. $\operatorname{null}\left(\boldsymbol{\Phi}_{k-1}\right)=$ $\mathbb{U}_{k: n}\left(\boldsymbol{\Psi}_{k-1}\right)$. If $k>n$, the square summation of the projections of $\left\{\boldsymbol{\varphi}_{s_{i}}\right\}_{i=1}^{k-1}$ onto the $\mu_{n} \operatorname{subspaces} \operatorname{span}\left(\mathbf{u}_{n-\mu_{n}+1}^{(k-1)}\right), \operatorname{span}\left(\mathbf{u}_{n-\mu_{n}+2}^{(k-1)}\right), \ldots$, and $\operatorname{span}\left(\mathbf{u}_{n}^{(k-1)}\right)$ are equal and minimum. Generally, $\lambda_{n}^{k-1}$ is a simple eigenvalue and $\mu_{n}=1$. If $\mu_{n}>1$ we 
can select the $k$-th observation vector that has the largest projection onto the spanned subspace $\operatorname{span}\left(\mathbf{u}_{n-\mu_{n}+1}^{(k-1)}, \mathbf{u}_{n-\mu_{n}+2}^{(k-1)}, \ldots, \mathbf{u}_{n}^{(k-1)}\right)$, which is exactly the minimum eigenspace of $\boldsymbol{\Psi}_{k-1}$.

In summary, for all $k \geq 1$, we can determine the $k$-th sensing location by maximizing the projection of the observation vector on the minimum eigenspace of the current dual observation matrix $\boldsymbol{\Psi}_{k-1}$.

For simplicity, we denote

$$
\mathbf{z}=\left(\mathbf{U}^{(k-1)}\right)^{\mathrm{T}} \boldsymbol{\varphi}_{s_{k}}
$$

where $\mathbf{U}^{(k-1)}=\left[\mathbf{u}_{1}^{(k-1)}, \mathbf{u}_{2}^{(k-1)}, \ldots, \mathbf{u}_{n}^{(k-1)}\right]$, and let $z_{i}$ be the $i$-th component of $\mathbf{z}$. It is clear that the square of the projection of $\boldsymbol{\varphi}_{s_{k}}$ onto the minimum eigenspace of $\Psi_{k-1}$ is

$$
\zeta_{k}=\sum_{i=n-\mu_{n}+1}^{n} z_{i}^{2}= \begin{cases}\sum_{i=k}^{n} z_{i}^{2} & \text { if } k<n \\ \sum_{i=n-\mu_{n}+1}^{n} z_{i}^{2} & \text { if } \quad k \geq n\end{cases}
$$

Let $\mathbf{P}_{k-1}$ be a projection matrix which can project any vectors in $\mathbb{R}^{n}$ onto the minimum eigenspace of $\boldsymbol{\Psi}_{k-1}$. When $k<n$, the minimum eigenspace of $\boldsymbol{\Psi}_{k-1}$ is the null space of $\boldsymbol{\Phi}_{k-1}$. Then, we can find that

$$
\mathbf{P}_{k-1}=\mathbf{I}_{n \times n}-\mathbf{R}_{k-1} \mathbf{R}_{k-1}^{\mathrm{T}}
$$

where

$$
\mathbf{R}_{k-1}=\operatorname{orth}\left(\Phi_{k-1}^{\mathrm{T}}\right)
$$

whose column vectors are obtained from the Gram-Schmidt Orthonormalization of all the column vectors of $\boldsymbol{\Phi}_{k-1}^{\mathrm{T}}$, i.e. the vector group $\left\{\boldsymbol{\varphi}_{s_{i}}\right\}_{i=1}^{k-1}$. When $k \geq n$, it is clear that

$$
\mathbf{P}_{k-1}=\mathbf{U}_{n}^{(k-1)}\left(\mathbf{U}_{n}^{(k-1)}\right)^{\mathrm{T}}
$$


where $\mathbf{U}_{n}^{(k-1)}=\left[\mathbf{u}_{n-\mu_{n}+1}^{(k-1)}, \mathbf{u}_{n-\mu_{n}+2}^{(k-1)}, \ldots, \mathbf{u}_{n}^{(k-1)}\right]$. Then we can obtain that

$$
\zeta_{k}=\left\|\mathbf{P}_{k-1} \boldsymbol{\varphi}_{s_{k}}\right\|_{2}^{2}
$$

Apparently $\zeta_{k}$ can be obtained if $\boldsymbol{\Phi}_{k-1}$ and $\boldsymbol{\varphi}_{s_{k}}$ are known, i.e. $\zeta_{k}$ satisfies the first condition of the new criterion used to replace $\lambda_{n}$. If $\zeta_{k} \neq 0$ for any $k<n$, the projection of $\boldsymbol{\varphi}_{s_{k}}$ onto $\operatorname{null}\left(\boldsymbol{\Phi}_{k-1}\right)$ is nonzero; therefore, $\boldsymbol{\varphi}_{s_{k}}$ is independent with all the vectors in $\left\{\boldsymbol{\varphi}_{s_{i}}\right\}_{i=1}^{k-1}$, which implies that $\zeta_{k}$ satisfies the second condition. Next, we utilize the following theorem to show that $\zeta_{k}$ satisfies the third condition.

Theorem 4. Given any observation matrix $\boldsymbol{\Phi}_{k}=\left[\boldsymbol{\varphi}_{s_{1}}, \boldsymbol{\varphi}_{s_{2}}, \ldots, \boldsymbol{\varphi}_{s_{k}}\right]^{\mathrm{T}} \in \mathbb{R}^{k \times n}$, and its corresponding dual observation matrix $\boldsymbol{\Psi}_{k}=\boldsymbol{\Phi}_{k}^{\mathrm{T}} \boldsymbol{\Phi}_{k}$ with a nonincreasing eigenvalue sequence $\left\{\lambda_{i}^{(k)}\right\}_{i=1}^{n}$.

1. If $k \leq n$ and $\boldsymbol{\Phi}_{k}$ is full row-rank, then

$$
\begin{aligned}
\lambda_{k}^{(k)} & =\frac{\zeta_{k}}{1+\sum_{i=1, z_{i} \neq 0}^{k-1} \frac{z_{i}^{2}}{\lambda_{i}^{(k-1)}-\lambda_{k}^{(k)}}} \\
\lambda_{k+1}^{(k)} & =\lambda_{k+2}^{(k)} \ldots=\lambda_{n}^{(k)}=0
\end{aligned}
$$

and $\lambda_{k}^{(k)}$ is monotonically increasing w.r.t. $\lambda_{k-1}^{(k-1)}$.

2. If $k \geq n$, then

$$
\begin{aligned}
& \lambda_{n-\mu_{n}+1}^{(k)}=\lambda_{n-\mu_{n}+1}^{(k-1)}+\frac{\zeta_{k}}{1+\sum_{i=1, z_{i} \neq 0}^{n-\mu_{n}} \frac{z_{i}^{2}}{\lambda_{i}^{(k-1)}-\lambda_{n-\mu_{n}+1}^{(k)}}} \\
& \lambda_{n-\mu_{n}+2}^{(k)}=\lambda_{n-\mu_{n}+3}^{(k)} \ldots=\lambda_{n}^{(k)}=\lambda_{n}^{(k-1)}
\end{aligned}
$$

and for any $M \geq n, \lambda_{n}^{(M)}$ is monotonically increasing w.r.t. $\zeta_{k}$ for all $k \leq M$.

Proof. See Appendix C. 
This theorem shows that $\lambda_{n}$ is monotonically increasing w.r.t. $\zeta_{k}$. Accordingly, WCEV is monotonically decreasing w.r.t. $\zeta_{k}$. It is clear that $\zeta_{k}$ can be another choice of the criterion instead of $\lambda_{n}$ to optimize the $k$-th sensing location. Therefore, to find the $k$-th sensing location, we maximize the magnitude of the projection of $\boldsymbol{\varphi}_{s_{k}}$ onto the minimum eigenspace of $\boldsymbol{\Psi}_{k-1}$ instead of maximizing the minimum nonzero eigenvalue of $\boldsymbol{\Psi}_{k}$. The greedy sensor placement algorithm, which we call maximal projection on minimum eigenspace (MPME), is given in Algorithm 2.

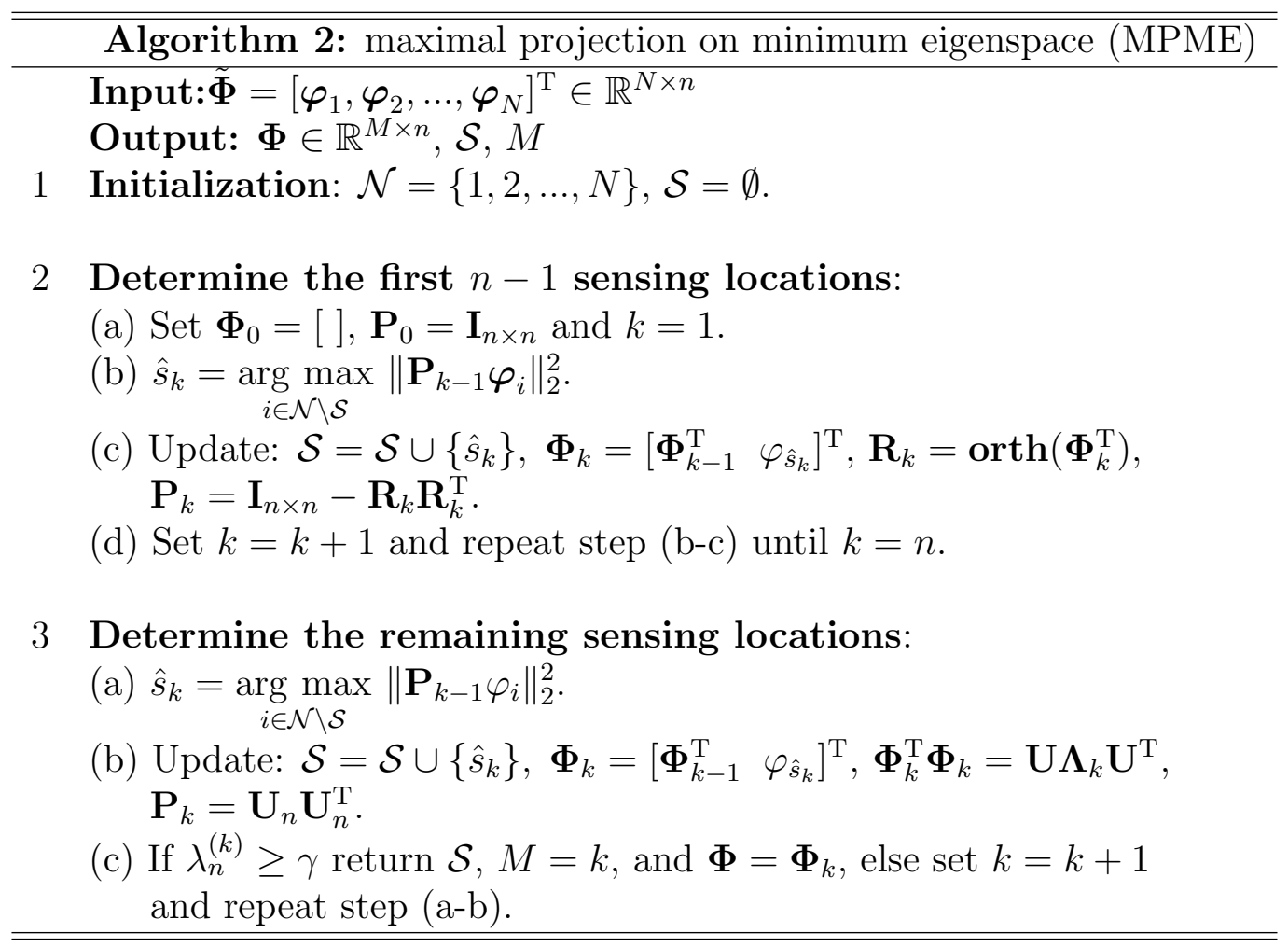

The $k$-th sensing location is obtained from the optimization problem in step 2(b) or step 3(a). To solve the optimization problem, we traverse all the unselected observation vectors and find the one that maximizes $\zeta_{k}$. Meanwhile, if $k \geq n$, we check the constraint in (4.7) after each sensing location is determined. If the constraint is satisfied, stop the algorithm. 


\subsubsection{Discussions about MNEP and MPME}

In both algorithms we need to solve the optimization problem in step 2(b) or step 3(a). In Algorithm 1, to solve the optimization problem, we need to evaluate the minimum nonzero eigenvalues of all $N-k+1$ possible dual observation matrices, while in Algorithm 2 we compute the projection of $N-K+1$ possible observation vectors onto the minimum eigenspace of $\boldsymbol{\Psi}_{k-1}$. The computational cost to find the eigenvalue of an $n \times n$ matrix is much more expensive than projecting one vector in $\mathbb{R}^{n}$ onto a known subspace. Therefore, the MPME is computationally much more efficient.

Equation (4.18a) shows that $\lambda_{k}^{(k)}$ is monotonic increasing w.r.t. $\zeta_{k}$ and decreasing w.r.t. $z_{i}^{2}$ for all $i<k \leq n$. Generally $\lambda_{n}^{(k-1)}$ is a simple eigenvalue when $k>n$, i.e. $\mu_{n}=1$, and (4.19a)-(4.19b) can be simplified as

$$
\lambda_{n}^{(k)}=\lambda_{n}^{(k-1)}+\frac{\zeta_{k}}{1+\sum_{i=1, z_{i} \neq 0}^{n-1} \frac{z_{i}^{2}}{\lambda_{i}^{(k-1)}-\lambda_{n}^{(k)}}}
$$

Equation (4.20) shows that $\lambda_{n}^{(k)}$ is monotonic increasing w.r.t. $\zeta_{k}$ and decreasing w.r.t. $z_{i}^{2}$ for all $i<n \leq k$. Therefore, the MNEP algorithm prefers to select the observation vector with large $\zeta_{k}$ and small $z_{i}^{2}$, which may not be with the largest $\zeta_{k}$ but achieves a balance between a large $\zeta_{k}$ and a small $z_{i}^{2}$. It is clear that $\sum_{i=1}^{n} z_{i}^{2}=\left\|\boldsymbol{\varphi}_{s_{k}}\right\|_{2}^{2}$ and $\sum_{i=1}^{k}\left\|\boldsymbol{\varphi}_{s_{i}}\right\|_{2}^{2}=\sum_{i=1}^{n} \lambda_{i}^{(k)}$; therefore, small $z_{i}^{2}$ means small increment of $\lambda_{i}^{(k)}$ from $\lambda_{i}^{(k-1)}$ for all $i<k$ when $k<n$, and all $i<n$ when $k \geq n$. Additionally, (4.18a) and (4.20) show that both $\lambda_{k}^{(k)}$ and $\lambda_{n}^{(k)}$ are monotonically increasing w.r.t. $\lambda_{i}^{(k-1)}$. Therefore, selecting the observation vector with small $z_{i}^{2}$ leads to a relatively smaller $\lambda_{k_{+}}^{\left(k_{+}\right)}$and $\lambda_{n}^{\left(k_{+}\right)}$, where $k_{+}>k$, and the MPME probably outperforms the MNEP in terms of finding the largest $\lambda_{n}^{(k)}$ for $k \geq n$.

In MPME we maximize $\zeta_{k}$, which can guarantee a large minimum nonzero eigenvalue of the updated dual observation matrix. In MNEP we directly maximize the minimum nonzero eigenvalue of the current dual observation matrix. Therefore, both algorithms guarantee that $\lambda_{n}^{(k)}$ is not much smaller than $\lambda_{i}^{(k)}$ for $i<n$ 
and $k \gtrsim n$, which implies that both algorithms can guarantee that $\boldsymbol{\Psi}_{k}$ is wellconditioned when $k \gtrsim n$. Here, $k \gtrsim n$ means $k$ is slightly larger than $n$.

In both algorithms, the minimum number of required sensor nodes is determined by judging whether $\lambda_{n}^{(k)} \geq \gamma$ is satisfied after each sensing location is determined when $k \geq n$. It is clear that in both algorithms the constraint in (4.7) is only considered in step 3(c), i.e. the last sub-step. In other words, the constraint in (4.7) is only used to judge whether the number of sensor nodes is enough.

Remark 11. We can change the constraint in (4.7) to other constraints described by MSE or $\operatorname{det}(\boldsymbol{\Sigma})$, if other measures are more interesting. Correspondingly, in step 3(c) we check the new constraint.

\subsection{Effectiveness of the MPME algorithm}

In this section, we provide four examples to illustrate the effectiveness of the MPME. The detailed analysis of the comparisons with the state-of-the-art, i.e. the convex relaxation [64], SparSenSe [69], and FrameSense [65] will also be presented.

Example 1. $\tilde{\boldsymbol{\Phi}} \in \mathbb{R}^{100 \times 20}$ is a Gaussian random matrix with independent components $\varphi_{i j} \sim \mathcal{N}(0,1)$, and the variance of the sensor noise $\sigma^{2}=1$.

Example 2. $\tilde{\boldsymbol{\Phi}} \in \mathbb{R}^{100 \times 20}$ is a Bernoulli random matrix with independent components $\varphi_{i j} \sim \mathcal{B}(1,0.5)$ with $\mathcal{B}$ representing the Binomial distribution, and the variance of the sensor noise $\sigma^{2}=1$.

The mean WCEV index and the mean MSE index of 200 Monte-Carlo simulation run results for the two examples are given in Figures 4.1-4.2. The optimization problems (4.9) and (4.10) are solved using the SDPT3 solver [113] and CVX toolbox [112], respectively. Actually, the SDPT3 solver is used as the computational engine of the CVX toolbox.

For the $i$-th $(1 \leq i \leq 200)$ simulation run, we determine the observation matrix $\boldsymbol{\Phi}_{k}^{(i)}$ $(20 \leq k \leq 40)$ based on the random signal representation matrix $\tilde{\boldsymbol{\Phi}}^{(i)}$, and obtain 
the following MSE index and WCEV index from (4.4) and (4.5), respectively:

$$
\begin{array}{r}
\operatorname{MSE}_{k}^{(i)}=\operatorname{tr}\left(\left(\Psi_{k}^{(i)}\right)^{-1}\right) \\
\mathrm{WCEV}_{k}^{(i)}=\lambda_{\max }\left(\left(\Psi_{k}^{(i)}\right)^{-1}\right)
\end{array}
$$

where $\boldsymbol{\Psi}_{k}^{(i)}=\left(\boldsymbol{\Phi}_{k}^{(i)}\right)^{\mathrm{T}} \boldsymbol{\Phi}_{k}^{(i)}$. Then, the mean MSE index and the mean WCEV index of 200 Monte-Carlo simulation run results are given by

$$
\begin{array}{r}
\overline{\mathrm{MSE}}_{k}=\frac{1}{200} \sum_{i=1}^{200} \mathrm{MSE}_{k}^{(i)} \\
\overline{\mathrm{WCEV}}_{k}=\frac{1}{200} \sum_{i=1}^{200} \mathrm{WCEV}_{k}^{(i)}
\end{array}
$$

It is shown in Figures 4.1-4.2 that for both examples, the MPME outperforms all the other methods in terms of the mean WCEV index and the mean MSE index. If the same number of sensor nodes are used, the MPME can provide the best results of linear inverse problems as compared with the other methods.

If we set the WCEV index threshold to be 0.3 (i.e. $\gamma=10 / 3$ ) for Example 1, Figure 4.1(A) shows that the minimum number of required sensor nodes $M_{\mathrm{MNEP}}=$ $M_{\mathrm{MPME}}=23, M_{\text {convex_relaxation }}=M_{\text {SparSenSe }}=28$, and $M_{\text {FrameSense }}=37$. If we set the MSE index threshold to be 1.5, Figure 4.1(B) shows that the minimum number of required sensor nodes $M_{\mathrm{MPME}}=23, M_{\mathrm{MNEP}}=25, M_{\text {convex_relaxation }}=$ $M_{\text {SparSenSe }}=26$, and $M_{\text {FrameSense }}=36$. Therefore, to meet the accuracy requirement, the proposed MPME algorithm requires the least number of sensor nodes. For the Bernoulli random data matrix we can easily obtain the same conclusion from Figure 4.2.

Figure 4.1 and Figure 4.2 show that MPME outperforms MNEP, which has been analyzed in Section 4.3.3. Additionally, we find that for all the five algorithms, the improvement of WCEV and MSE are increasingly insignificant with an increase in the number of sensor nodes. It means that the influence of additional sensor observation declines and its location is not so critical as the previously determined sensing locations. It is clearly shown in Figure 4.1 and Figure 4.2 that MPME is 


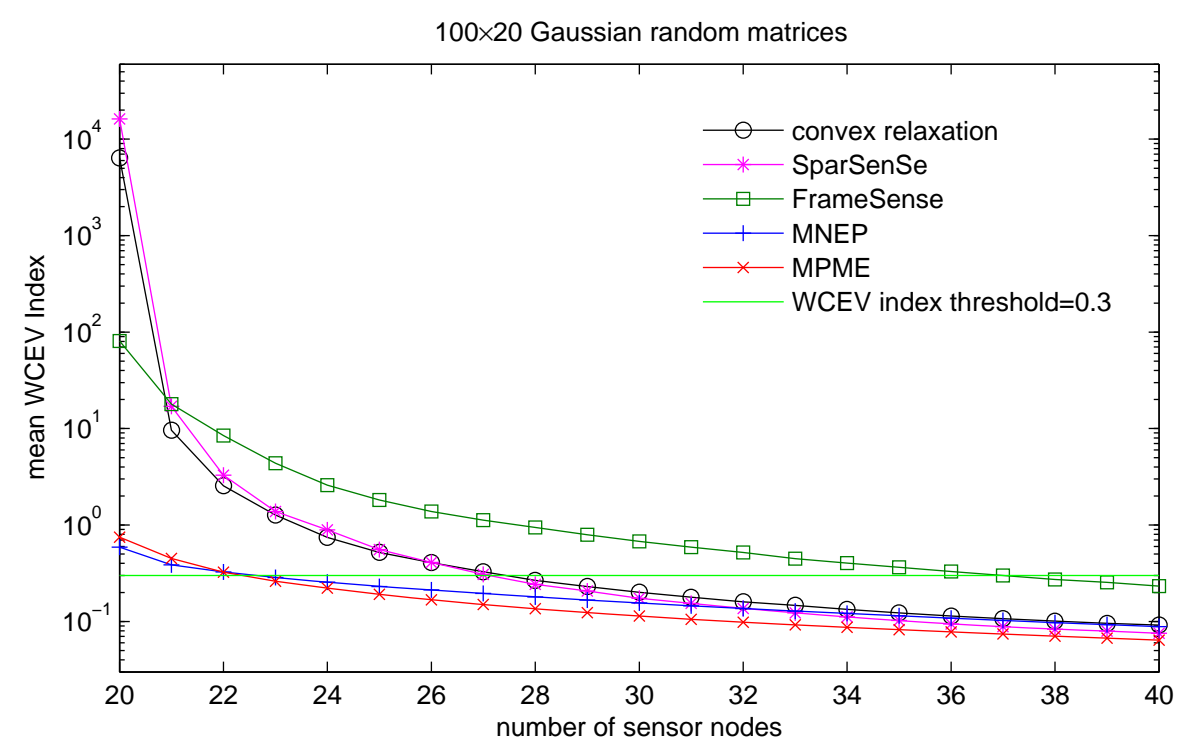

(A) mean WCEV index

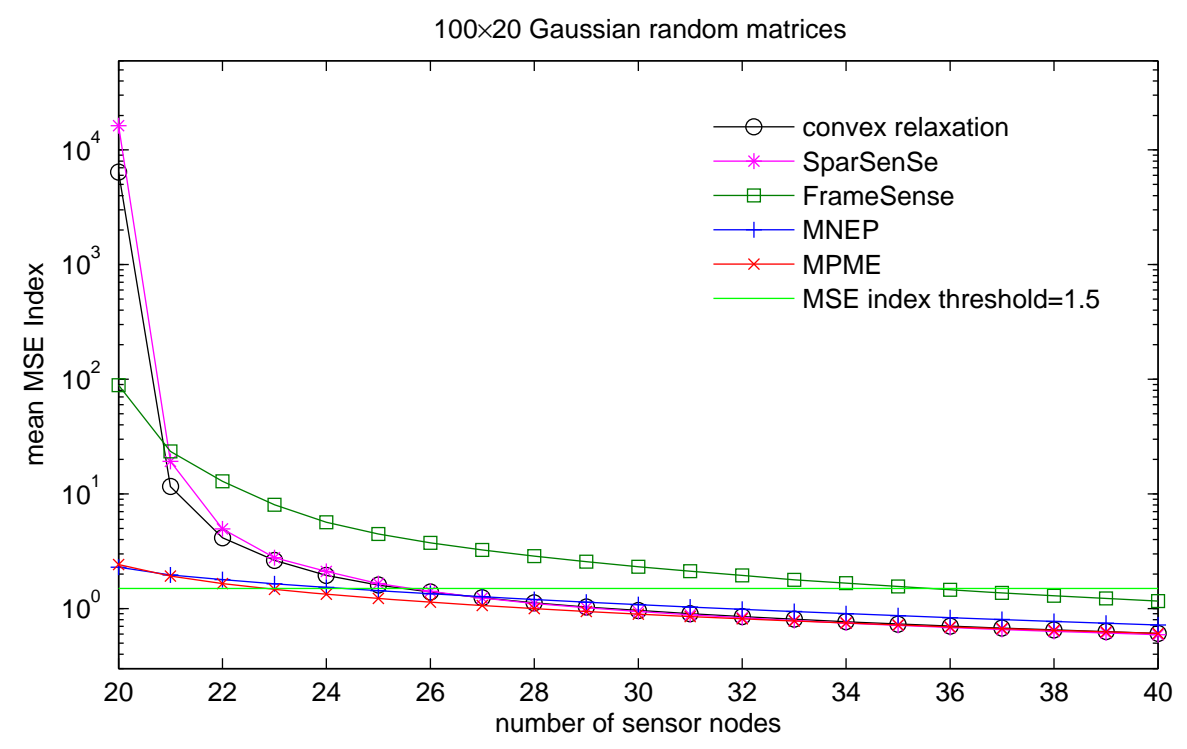

(B) mean MSE index

FiguRE 4.1: The performance comparison between the MPME and other sensor placement methods for Example 1. For the MPME and the MNEP we do not consider the stopping criteria but show the mean WCEV index and the mean MSE index w.r.t. the number of sensor nodes, increasing from 20 to 40 . For the SparSenSe method, we do not consider the minimum number of required sensor nodes but determine the sensing locations like the convex relaxation method by finding the indices of the $M$ largest elements of $\mathbf{w}^{*}$.

much better than the convex relaxation method, SparSenSe, and FrameSense when the number of sensor nodes is very limited, i.e. the MPME method significantly outperforms the state-of-the-art in finding the critical sensing locations, which is 


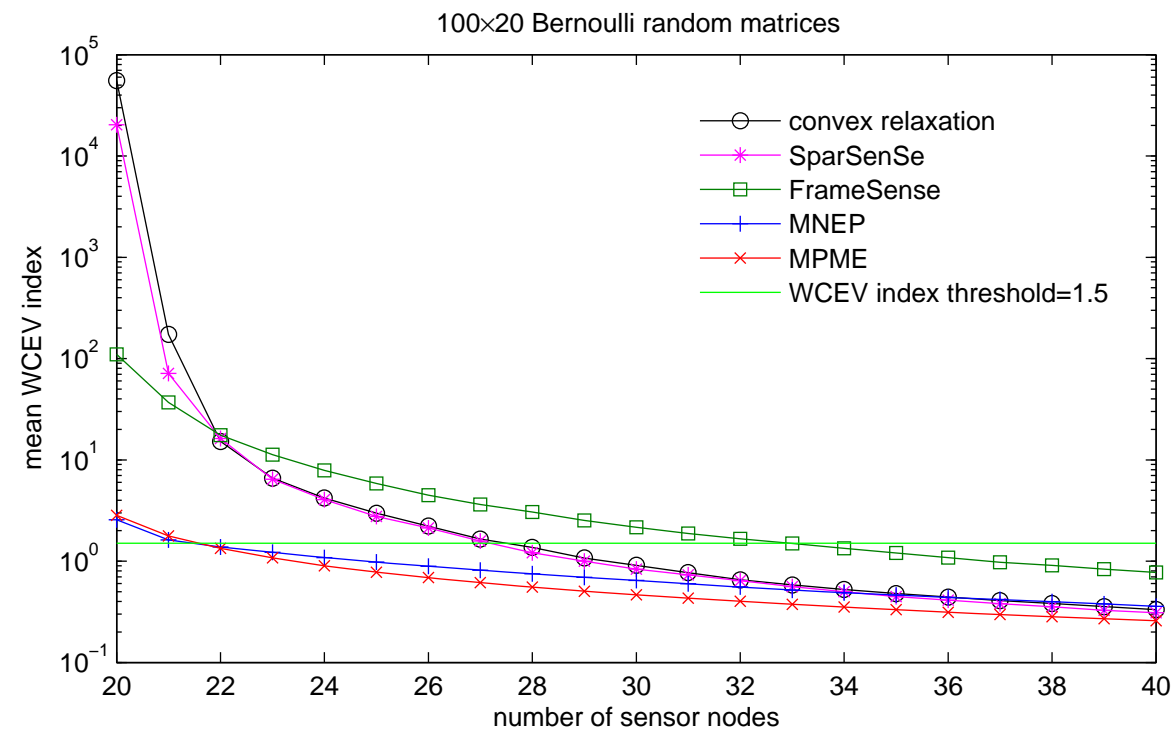

(A) mean WCEV index

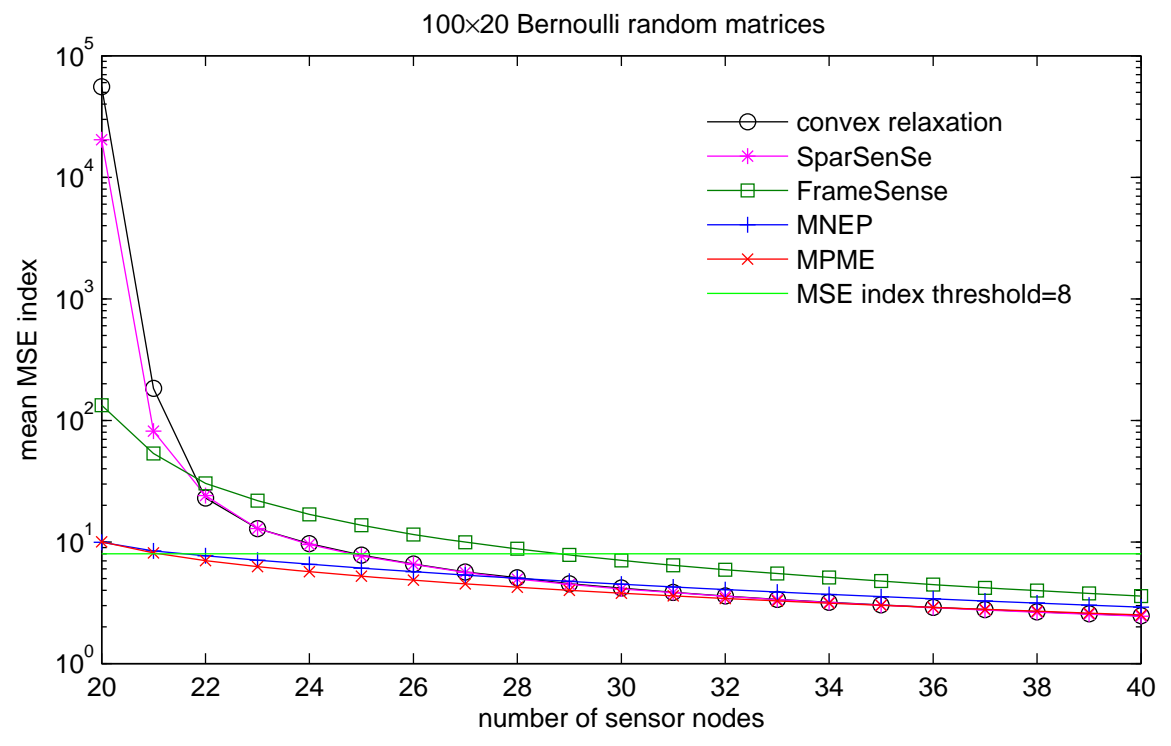

(B) mean MSE index

FiguRE 4.2: The performance comparison between the MPME and other sensor placement methods for Example 2. For the MPME and the MNEP we do not consider the stopping criteria but show the mean WCEV index and the mean MSE index w.r.t. the number of sensor nodes, increasing from 20 to 40 . For the SparSenSe method, we do not consider the minimum number of required sensor nodes but determine the sensing locations like the convex relaxation method by finding the indices of the $M$ largest elements of $\mathbf{w}^{*}$.

analyzed as follows. 


\subsubsection{Comparison with convex relaxation}

Figure 4.1 and Figure 4.2 shows that the mean MSE index and the mean WCEV index of the convex relaxation method are much larger than those of the MPME method when the number of sensor nodes is slightly larger than the dimension of the vector to be estimated, i.e. when $k \gtrsim n=20$ in this example.

Comparing Figure 4.1(A) with Figure 4.1(B), we find that when using the convex relaxation method, the $\mathrm{WCEV}$ index (i.e. the maximum eigenvalue of $\boldsymbol{\Psi}_{k}^{-1}$, see Equation (4.22)) contributes the main part of the MSE index (i.e. the trace of $\boldsymbol{\Psi}_{k}^{-1}$, see Equation (4.21)), especially when the number of sensor nodes is small. Comparing Figure 4.2(A) with Figure 4.2(B), we can obtain the same conclusion. It means that the maximum eigenvalue of $\boldsymbol{\Psi}_{k}^{-1}$ is overwhelmingly larger than the others when $k \gtrsim n$. In other words, the minimum eigenvalue of $\boldsymbol{\Psi}_{k}$ is much smaller than the other eigenvalues, which implies that $\boldsymbol{\Psi}_{k}$ is ill-conditioned, and hence the estimated vector $\hat{\boldsymbol{\alpha}}$ is inaccurate.

The mean condition number of Monte-Carlo simulation result for Example 1 is shown in Figure 4.3. For $i$-th $(1 \leq i \leq 200)$ simulation run, the condition number of $\boldsymbol{\Psi}_{k}^{(i)}$ is denoted by $\kappa\left(\Psi_{k}^{(i)}\right)$. The mean condition number is given by

$$
\bar{\kappa}_{k}=\frac{1}{200} \sum_{i=1}^{200} \kappa\left(\Psi_{k}^{(i)}\right)
$$

Figure 4.3 shows that for $k \leq 23, \boldsymbol{\Psi}_{k}$ obtained from the convex relaxation method is ill-conditioned, whereas $\boldsymbol{\Psi}_{k}$ obtained from MNEP or MPME is well-conditioned. Accordingly, as shown in Figure 4.1 and Figure 4.2, the mean MSE index and the mean WCEV index of the convex relaxation method are much larger than those of MPME, respectively.

The solution of (4.8) should always lead to a well-conditioned $\boldsymbol{\Psi}$. However, the cardinality optimization problem (4.8) cannot be directly solved. In practice, we use the solution of the convex optimization problem (4.9) instead. The solution of (4.9), $\mathbf{w}^{*} \in[0,1]^{N}$, is mapped into $\{0,1\}^{N}$ to find the sensing locations. The largest 


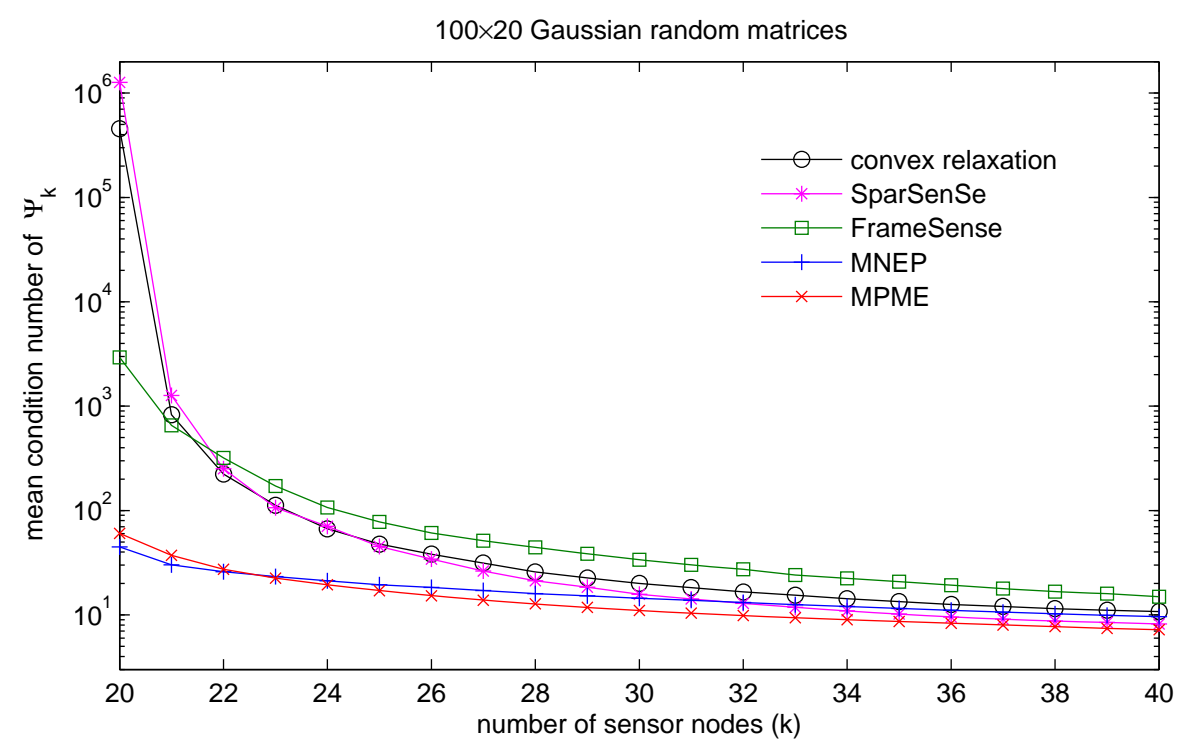

FiguRE 4.3: The mean condition number of the dual observation matrix $\boldsymbol{\Psi}_{k}=\boldsymbol{\Phi}_{k}^{\mathrm{T}} \boldsymbol{\Phi}_{k}$ for Example 1. Here $\boldsymbol{\Phi}_{k}$ is obtained from the five sensor placement algorithms.

$M$ elements of $\mathbf{w}^{*}$ are mapped to 1 and other elements are mapped to 0 . In such a mapping, the singularity of $\boldsymbol{\Psi}$ is not considered, and the result of the mapping is different with the solution of (4.8). The number of sensor nodes is nearer the dimension of the estimated vector $\hat{\boldsymbol{\alpha}}$, giving a higher probability of $\boldsymbol{\Psi}$ being illconditioned. However, the proposed MNEP and MPME algorithms can guarantee a large minimum nonzero eigenvalue of $\boldsymbol{\Psi}_{k}$ and accordingly a well-conditioned $\boldsymbol{\Psi}_{M}$ for $M \geq n$.

\subsubsection{Comparison with SparSenSe}

It is claimed that SparSenSe can determine the minimum number of required sensor nodes by utilizing the sparsity of the variable $\mathbf{w}$ in (4.10). With a predefined threshold $\tau$ and the solution of optimization problem (4.10), i.e. $\mathbf{w}^{*}$, if $w_{i}^{*}<\tau$ set $w_{i}^{*}=0$. The sensing indices then exactly correspond to the nonzero entries of $\mathbf{w}^{*}$ and the number of nonzero entries is the minimum number of required sensor nodes. This strategy works well for the example in [69] in which $\tilde{\boldsymbol{\Phi}} \in \mathbb{R}^{50 \times 2}$, and the largest 3 entries of $\mathbf{w}^{*}$ are much larger than other elements. We can set $\tau$ as 
a small value and easily find the largest 3 entries corresponding to the selected sensing indices.

However, this strategy is ineffective if the dimension of the estimated vector $\boldsymbol{\alpha}$ is large. In the pervious two examples, w $\in \mathbb{R}^{100}$ with at least 20 nonzero entries is not sparse. We introduce another example to illustrate this problem.

Example 3. $\tilde{\boldsymbol{\Phi}} \in \mathbb{R}^{1500 \times 20}$ is a Gaussian random matrix with independent components $\varphi_{i j} \sim \mathcal{N}(0,1)$. The variance of the sensor noise $\sigma^{2}=1$, and the maximum acceptable MSE index $\gamma^{\prime}=1.5$.

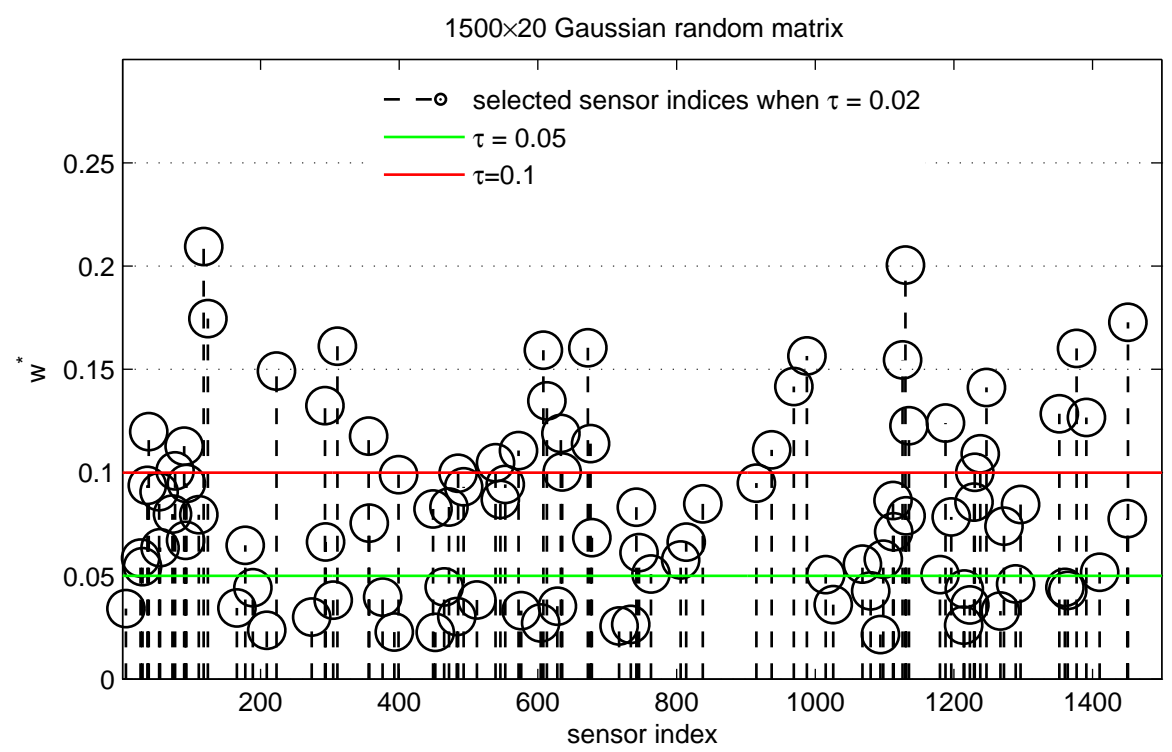

FiguRE 4.4: Selected sensing indices with SparSenSe for Example 3.

In Example 3, $N \gg n$ guarantees that the decision variable $\mathbf{w}$ is sparse. Figure 4.4 shows that if $\tau=0.02,98$ sensor nodes are selected. If $\tau$ is set as 0.05 and 0.1 , then 71 and 31 sensor nodes are selected, respectively. We can see the result of SparSenSe from Figure 4.5 that 31 sensor nodes can guarantee that the MSE index is less than the maximum acceptable MSE index. However, the minimum number of required sensor nodes is 23. For this example, if we set $\tau=\tau^{*} \in[0.1129,0.1140]$, the 23 largest elements of $\mathbf{w}^{*}$ will be selected. In practice, however, the optimal threshold $\tau^{*}$ is a prior unknown; therefore, utilizing the sparsity of $\mathbf{w}$ to determine the minimum number of sensor nodes is ineffective. 


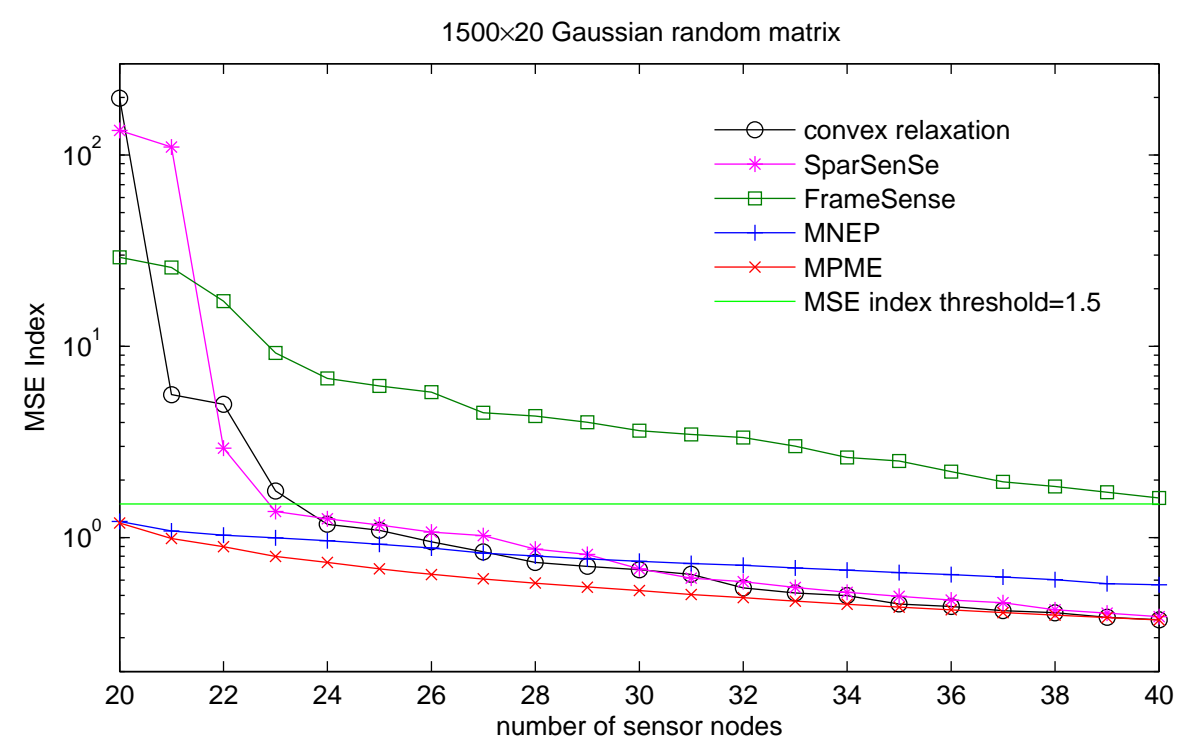

Figure 4.5: The MSE index of $\hat{\boldsymbol{\alpha}}$ estimated from 20 to 40 sensor observations for Example 3.

In practice, we can set $M=n$ and select the sensing indices that correspond to the $M$ largest entries of $\mathbf{w}^{*}$. Then, we check whether the accuracy (i.e. WCEV index or MSE index) is acceptable. If not, increase $M$, reselect the sensing indices and recheck the accuracy until the accuracy is acceptable. Using this strategy for Example 3, we can easily find the minimum number of required sensor nodes $M=23$.

Like the convex relaxation method, Figures 4.1-4.2 and Figure 4.5 show that the solutions of MPME are much better than those of SparSenSe when $k \gtrsim n$. The reason is that the sensing indices corresponding to the $k$ largest elements of $\mathbf{w}^{*}$ cannot guarantee a well-conditioned $\boldsymbol{\Psi}_{k}$ as shown in Figure 4.3.

\subsubsection{Comparison with FrameSense}

It is apparent from Figure 4.1-4.2 and Figure 4.3 that FrameSense provides the worst results for the first two examples in which $\tilde{\boldsymbol{\Phi}}$ is not an equal-norm frame, i.e. the norms of the rows of $\tilde{\boldsymbol{\Phi}}$ are not equal. For these cases, minimization of the frame potential in (4.11) will select the rows of $\tilde{\boldsymbol{\Phi}}$ with small norms to construct the observation matrix $\boldsymbol{\Phi}$. FrameSense prefers to drop the rows with large norms 
[65]. It is easily found that

$$
\sum_{i=1}^{M}\left\|\boldsymbol{\varphi}_{s_{i}}\right\|^{2}=\operatorname{tr}(\Psi)=\sum_{i=1}^{n} \lambda_{i}
$$

Compared this equation with the MSE in (4.4), we conclude that small norms of the rows of the observation matrix $\boldsymbol{\Phi}$ lead to a large MSE of the estimated vector $\hat{\boldsymbol{\alpha}}$. From another perspective, we find that the smaller the norm of $\boldsymbol{\varphi}_{s_{i}}$ for $i=1, \ldots, M$, the smaller is the signal-to-noise ratio of the measurement model. Therefore, FrameSense is only suitable for the case where $\tilde{\boldsymbol{\Phi}}$ corresponds to an equal-norm frame.

Actually, even if $\tilde{\boldsymbol{\Phi}}$ corresponds to an equal-norm frame, the proposed MPME algorithm still outperforms FrameSense, which will be illustrated by the following example.

Example 4. $\tilde{\boldsymbol{\Phi}} \in \mathbb{R}^{100 \times 20}$ is a random matrix with normalized rows, whose $i$-th row $\varphi_{i}^{\mathrm{T}}=\frac{\phi_{i}^{\mathrm{T}}}{\left\|\phi_{i}\right\|}$, and $\boldsymbol{\phi}_{i} \in \mathbb{R}^{20}$ is a random vector with independent components $\phi_{i j} \sim \mathcal{N}(0,1)$. The variance of sensor noise $\sigma^{2}=1$.

The mean WCEV index and the mean MSE index of 200 Monte-Carlo run results are shown in Figure 4.6. Figure 4.6 shows that FrameSense outperforms the convex relaxation method and SparSenSe when the number of sensor nodes is small. The bottom figure shows that the five methods except MNEP provide almost the same mean MSE indices when the number of sensor nodes is large enough, which indicates the effectiveness of FrameSense in pursuing the minimum MSE.

However, like the convex relaxation method and SparSenSe, in Figure 4.6, both WCEV and MSE of FrameSense are much larger than those of MPME when the number of sensor nodes is small (e.g., $k=20$ or 21 ), which indicates that FrameSense cannot guarantee a well-conditioned $\boldsymbol{\Psi}_{k}$ when $k \gtrsim n$.

Additionally, Figure 4.6 shows that MPME requires the least number of sensor nodes to meet the accuracy requirement, and that the required sensor nodes of FrameSense is less than those of the convex relaxation method and SparSenSe. 


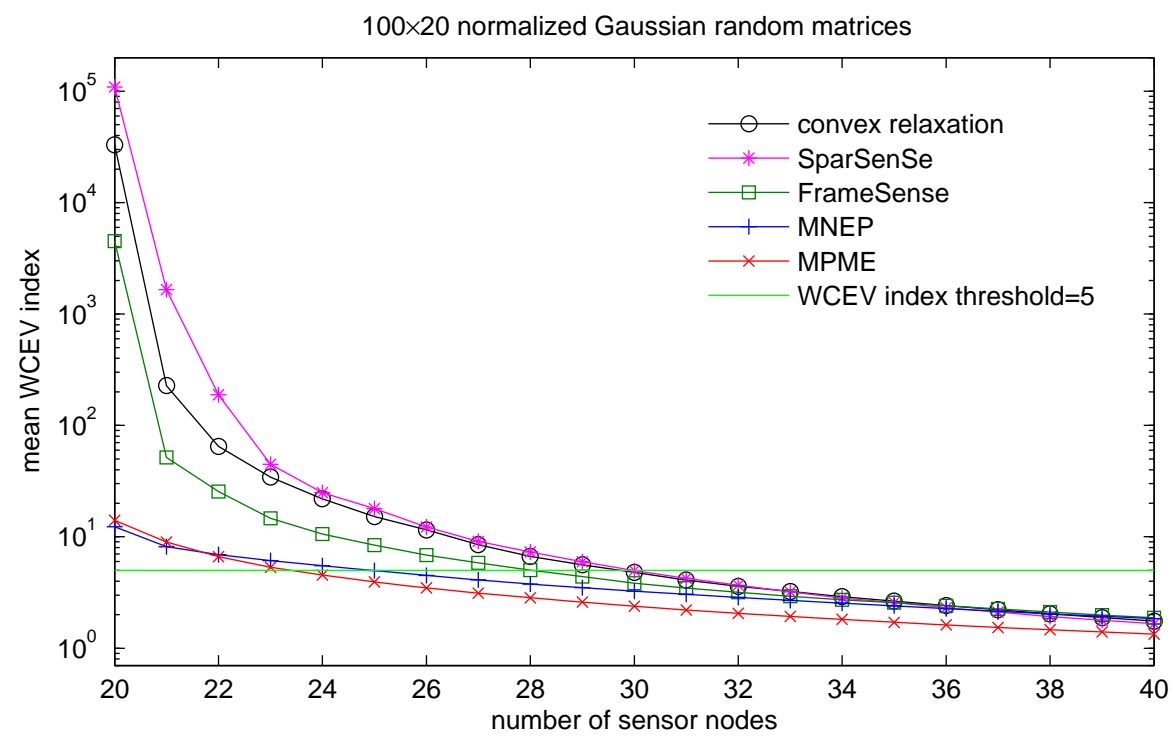

(A) mean WCEV index

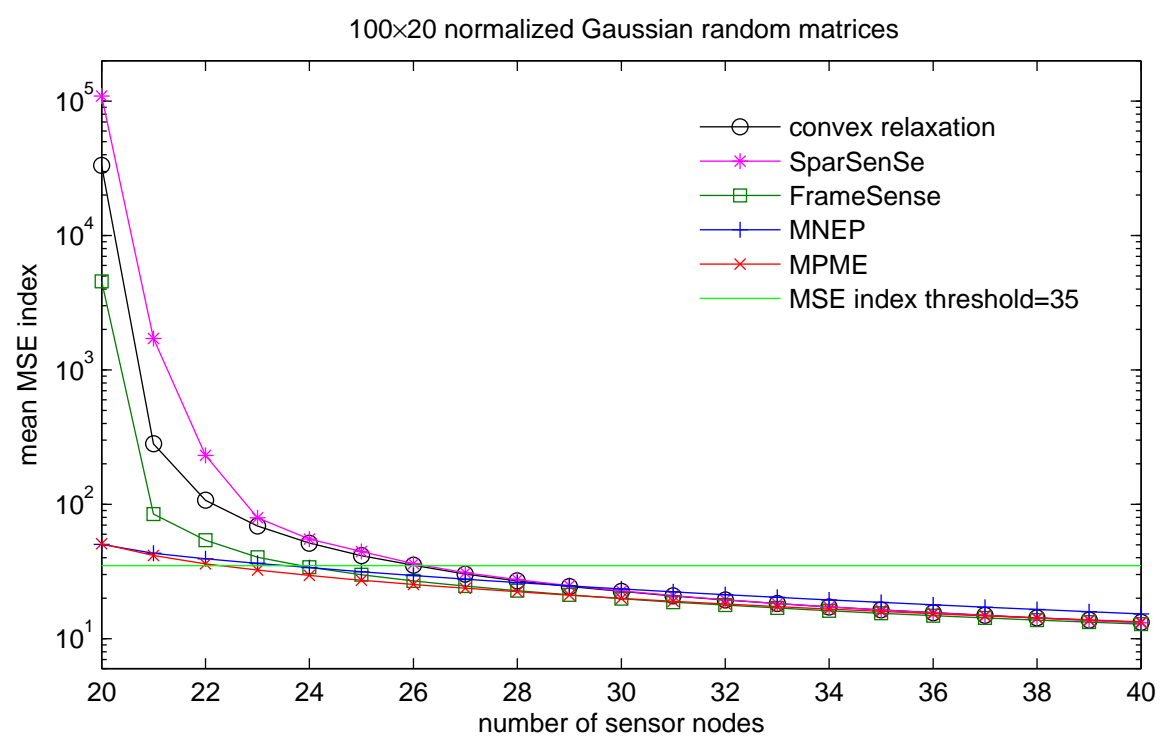

(B) mean MSE index

Figure 4.6: The mean WCEV index and mean MSE index of $\hat{\boldsymbol{\alpha}}$ estimated from 20 to 40 sensor observations for Example 4.

If $\tilde{\boldsymbol{\Phi}}$ corresponds to an equal-norm frame, the minimum frame potential of $\boldsymbol{\Phi}$ implies the minimum MSE, but the "worst-out" strategy in FrameSense cannot find the optimal solution, and even cannot guarantee that $\boldsymbol{\Psi}$ is well-conditioned if the available sensor nodes is limited. The solution of FrameSense is near-optimal because of the sub-modularity of the cost function; however our MPME algorithm still outperforms FrameSense in the four examples. Hence, our future work may 
focus on exploring the reasons why MPME outperforms the near-optimal solution from a theoretical perspective.

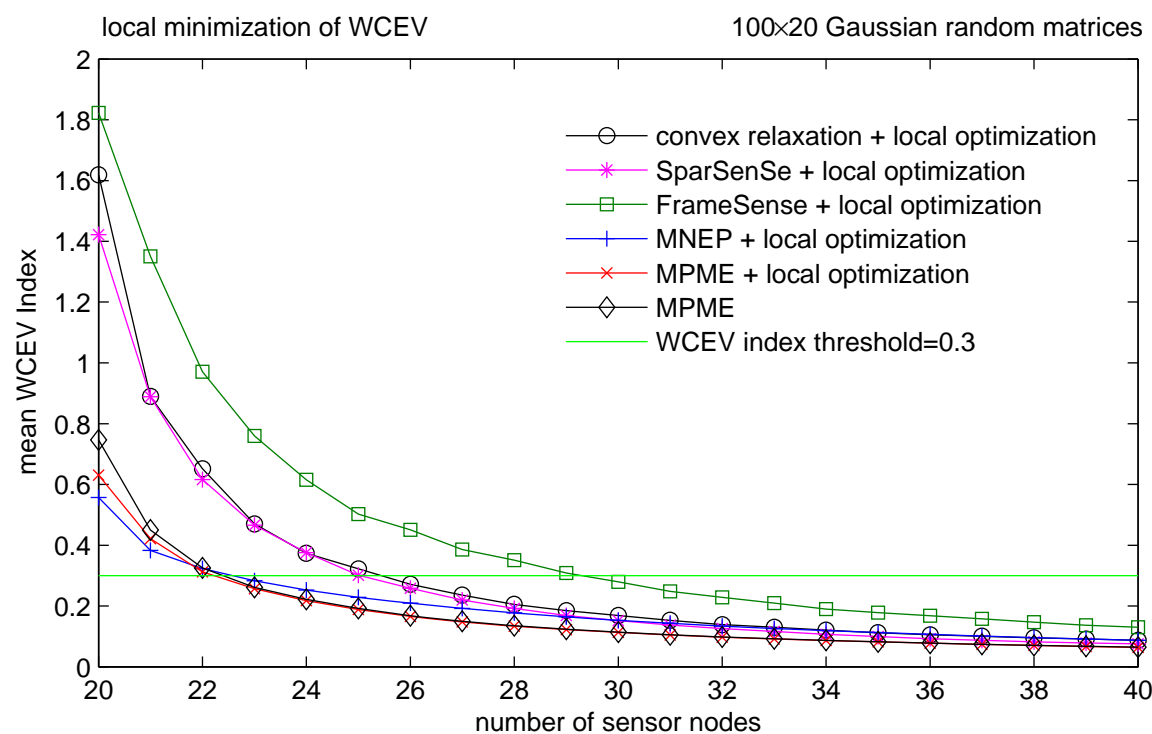

(A) mean WCEV index

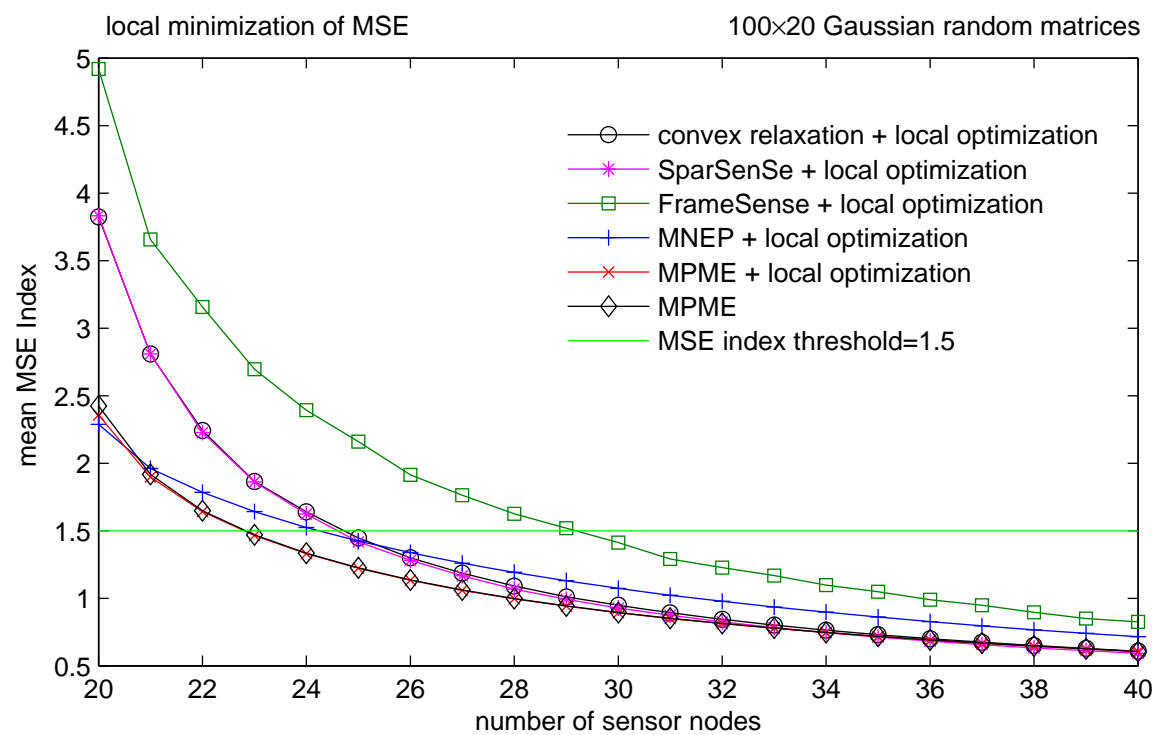

(B) mean MSE index

FiguRE 4.7: The performance comparison between MPME and five methods with local optimization for Example 1 


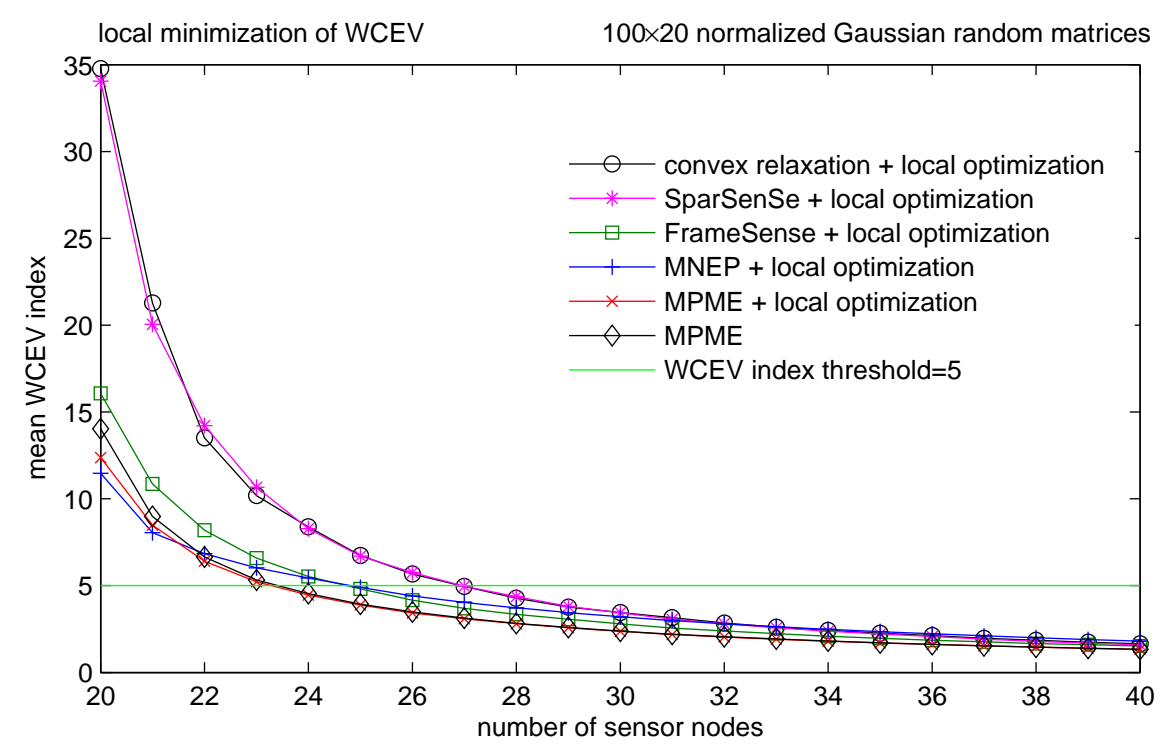

(A) mean WCEV index

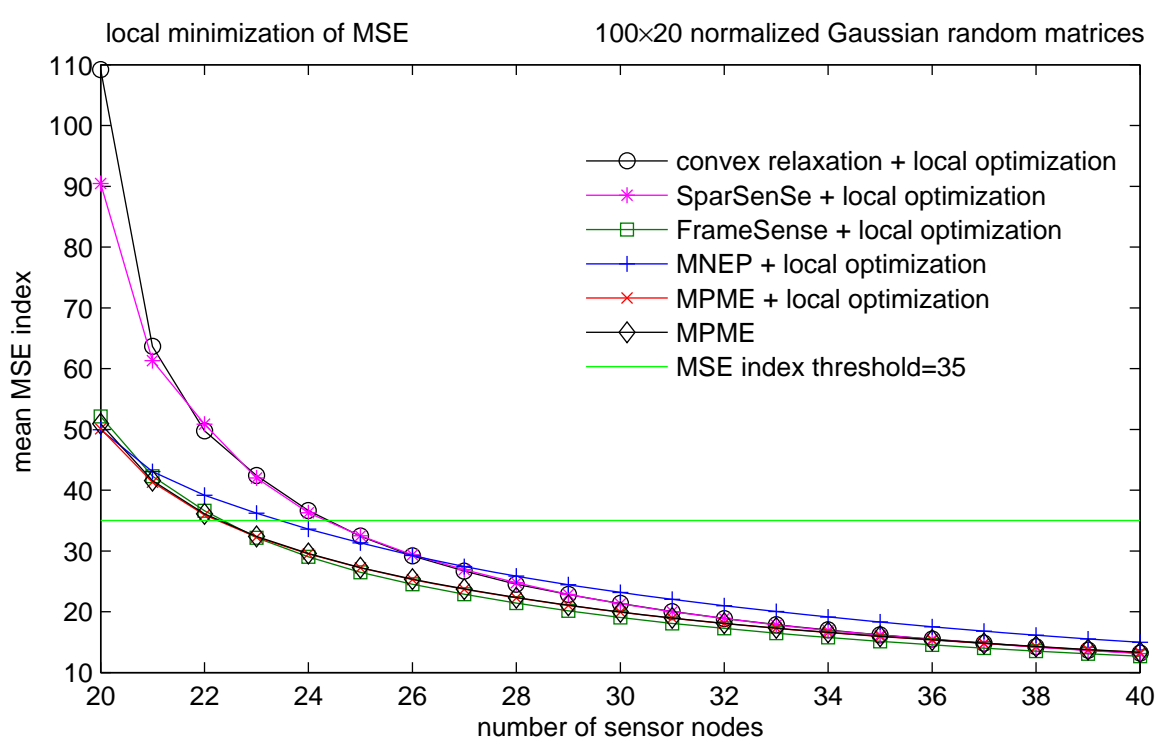

(в) mean MSE index

FiguRE 4.8: The performance comparison between MPME and five methods with local optimization for Example 4.

\subsubsection{Local optimization}

The four examples show that the current methods are not suitable for the cases when the number of sensors is small. This drawback can be overcome by a computationally expensive technique, i.e. the so called local optimization technique [64]. 
Definition 2 (Local optimization). For a given set of sensing locations $\mathcal{S}$, exchange one-at-a-time all the sensing location in $\mathcal{S}$ with each available candidate location in $\mathcal{N} / \mathcal{S}$ to re-locate the sensor nodes at new position that can further reduce one criterion of interest (e.g., MSE or WCEV) until there is no further decrease.

This technique is similar with Fedorov's exchange algorithm [114], and Wynn's algorithm [115]. It has also been discussed in [64, 67]. For any results of local optimization, replacing any selected sensing location by any unselected one cannot improve the solution, which is called 2-opt.

We apply the local optimization technique to the solutions obtained from the five methods for Example 1 and Example 4. Figures 4.7-4.8 show the mean WCEV indices and the mean MSE indices of the improved sensor configurations, together with those directly obtained from MPME, i.e. without local optimization. In Figures 4.7-4.8 the solutions of the convex relaxation method, SparSenSe, and FrameSense are remarkably improved by the local optimization, especially when the number of sensor nodes is small. However, the solutions of MPME almost have no improvement. Nevertheless, Figure 4.7 shows that the solution of MPME without local optimization still outperforms all the other solutions with local optimization in terms of both indices.

Figures 4.8(B) shows that with local optimization, the solution of FrameSense for normalized Gaussian random matrices are sightly better than the solution of MPME without local optimization. However, the local optimization is computationally very expensive, and the MPME provides the best result amongst the five methods for all the other cases. How the local optimization affects a given sensor configuration and which types of sensor configuration can be greatly improved by local optimization are still open problems.

Additionally, Figure 4.7-4.8 show that for all cases, to meet the accuracy requirement, the solutions of MPME without local optimization require the least number of sensor nodes. From this perspective, MPME without local optimization outperforms the state-of-the-art with local optimization. 


\subsection{Computational cost of the MPME algorithm}

In this section, we compare the computational cost of MPME with that of the state-of-the-art.

The computational effort of the convex relaxation method is $O\left(i_{\mathrm{c}} N^{3}\right)$ [64]. The convex optimization problem is solved using the interior-point method and $i_{\mathrm{c}}$ is the iteration number Typically, the iteration number is of a few tens [64].

Similar to the convex relaxation method, the computational effort of SparSenSe is $O\left(i_{s} N^{3}\right)$ where $i_{s}$ is the iteration number of solving the convex optimization problem (4.10).

When using FrameSense, $N-M$ rows are removed from $\tilde{\boldsymbol{\Phi}}$. It costs $O\left((N-k+1)^{2}\right)$ to determine the $k$-th removed row. Since $M \ll N$, the total cost of FrameSense is $O\left(\sum_{i=1}^{N} i^{2}-\sum_{i=1}^{M} i^{2}\right)=O\left(N^{3}\right)$.

Finding the eigenvalues of $\mathbf{\Psi}_{k}$ costs $O\left(n^{3}\right)$ operations. When we find the $k$-th sensing location via MNEP, the main computational cost is to solve the minimum nonzero eigenvalue maximization problem in which $N-k+1$ eigenvalue problems are solved. The computation cost is $O\left(N n^{3}\right)$. Therefore, to determine all the $M$ sensing locations via MNEP, the total computational effort is $O\left(N M n^{3}\right)$.

To determine the $k$-th sensing location via MPME, the main computational cost is attributed to the optimization problem $\hat{s}_{k}=\underset{i \in \mathcal{N} \backslash \mathcal{S}}{\arg \max }\left\|\mathbf{P}_{k-1} \boldsymbol{\varphi}_{i}\right\|_{2}$ which costs $O\left((N-k+1) n^{2}\right)$. Hence, finding all the $M$ sensing locations costs $O\left(N M n^{2}\right)$.

We summarize the computational efforts of the five methods in Table 4.1. The mean computation time of 50 simulation run results are shown in Figure 4.9. Both Table 4.1 and Figure 4.9 show that MPME is computationally the most efficient one amongst the five algorithms if $N \gg M \geq n$. 
TABLE 4.1: The computational effort of the five sensor placement methods

\begin{tabular}{ccccc}
\hline \hline Convex Relaxation & SparSenSe & FrameSense & MNEP & MPME \\
\hline$O\left(i_{\mathrm{c}} N^{3}\right)$ & $O\left(i_{s} N^{3}\right)$ & $O\left(N^{3}\right)$ & $O\left(N M n^{3}\right)$ & $O\left(N M n^{2}\right)$ \\
\hline \hline
\end{tabular}

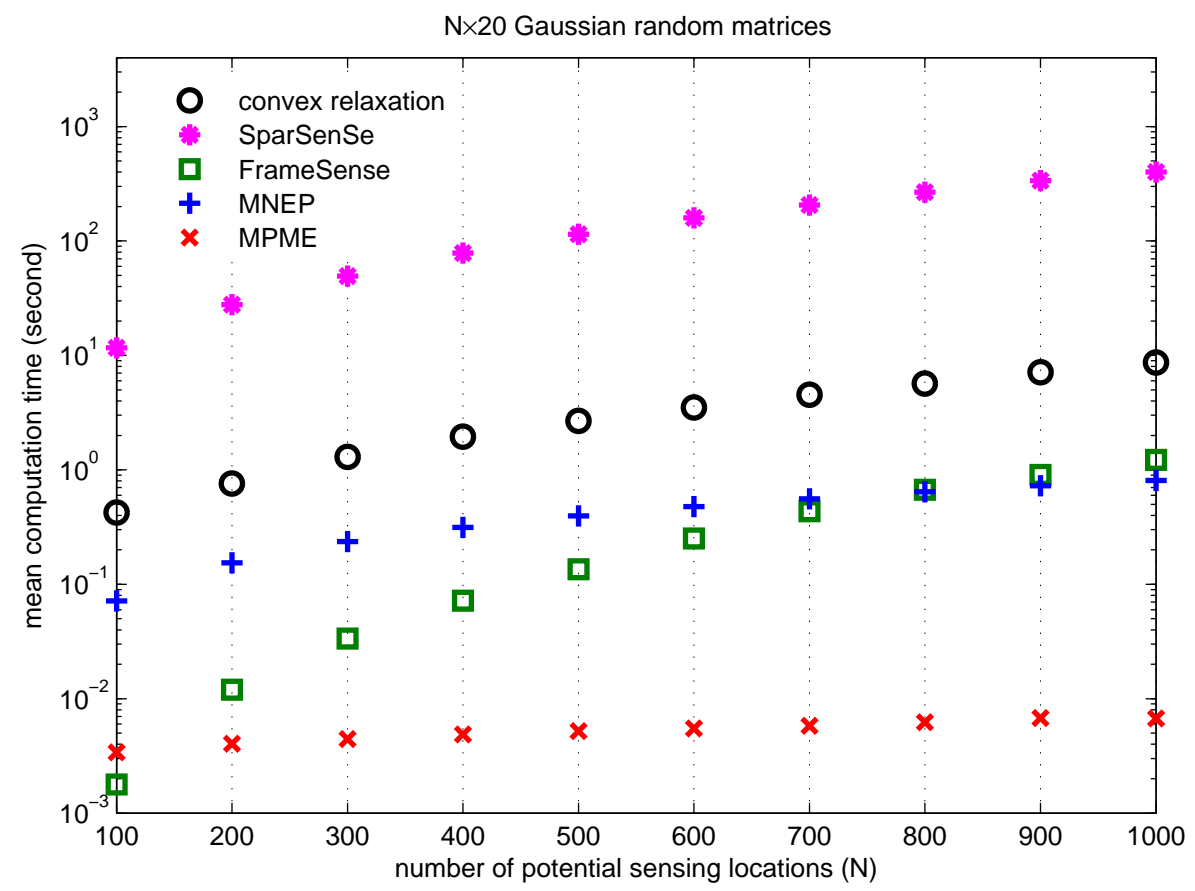

Figure 4.9: The mean computation time of the five sensor placement algorithms for $N \times 20$ Gaussian random matrices where $N$ is from 100 to 1000 . In the simulations, the number of available sensor nodes, i.e. $M$, is set as 20 for the convex relaxation method, FrameSenSe, MNEP and MPME. The computation time is estimated by the MATLAB tic-toc commands. The simulation is run in a laptop with a $2.4 \mathrm{GHz}$ Intel $\mathrm{i} 3-3110 \mathrm{M}$ processor. The mean computation time is the mean value of 50 different simulations, and for each simulation, $\tilde{\boldsymbol{\Phi}} \in \mathbb{R}^{N \times 20}$ with independent entries $\varphi_{i j} \sim \mathcal{N}(0,1)$.

\subsection{Storage requirement of the MPME algorith-}

\section{$\mathbf{m}$}

We used the proposed MPME method to determine the sensing locations of the indoor thermal map reconstruction problem in Chapter 3. The proposed MPME can immediately provide the solution in a laptop with 2GB RAM. The solution is shown in Figure 3.12. For easy reference, we show it in Figure 4.10. 


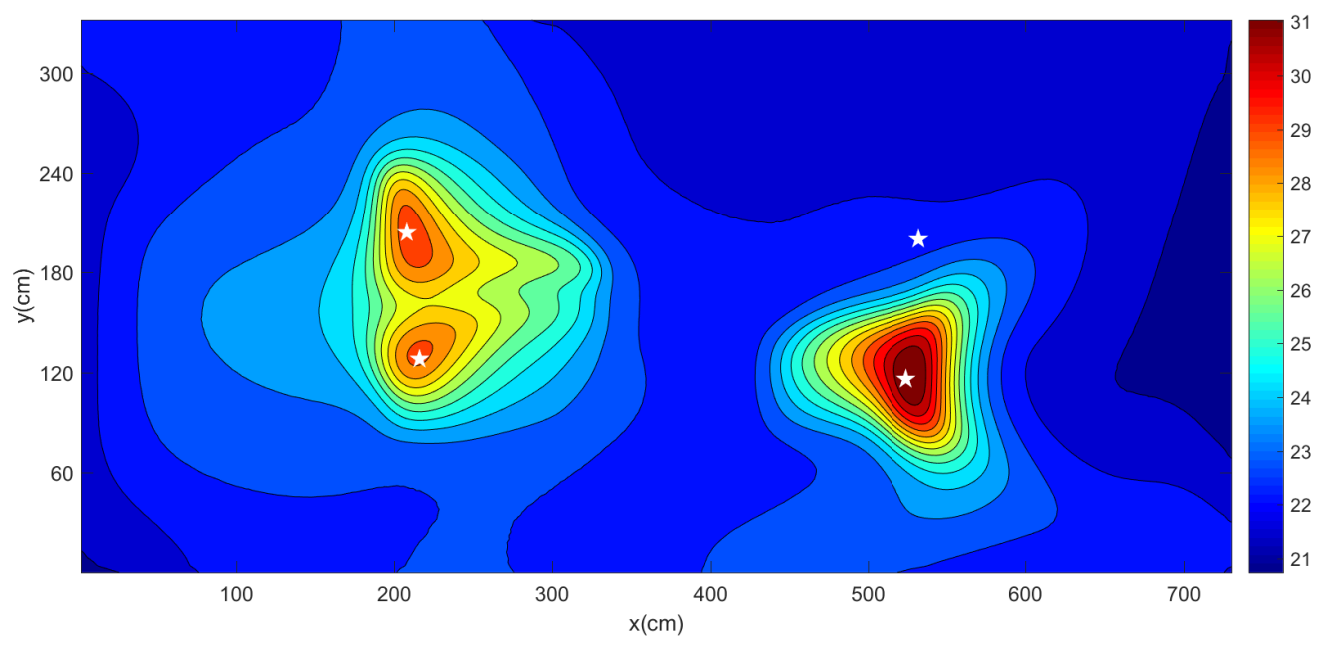

FiguRE 4.10: The estimated thermal map using the proposed TSPFR method. The stars represent the sensing locations obtained from the proposed MPME algorithm. The signal representation matrix $\tilde{\boldsymbol{\Phi}}$ is obtained from the domiannt PCA modes.

However, for this example, the convex relaxation, the SparSenSe and the FrameSense methods cannot work in a workstation with 16GB RAM due to the shortage of memory. In this example, the number of the potential sensing locations $N=167 \times 365=60955$, which is too large for the state of the arts to solve the sensor placement problem on such a workstation.

The state of the arts (convex relaxation, SparSenSe, and FrameSense) require to storage a $N \times N$ matrix. By contrast, the proposed MNEP and MPME algorithms only require to storage a $n \times n$ matrix. $N$ is usually a very large number, especially for 3-dimensional physical field reconstruction. In many 3-dimension CFD applications, the number of mesh points $(N)$ is commonly up to tens of millions. However, $n$ is usual a small number, as we discussed in Chapter 3, it is always smaller than the number of sensor observations. In this example, $n=4$. Therefore, we conclude that the storage requirement of the proposed MNEP and MPME algorithms is much less than the state of the arts.

If $N$ is a large number, both the computational cost (as can be seen in Figure 4.9) and the storage requirement of the state of the arts will dramatically increase, 
which probably makes the sensor placement problem unsolvable via the state of the arts.

The storage requirement of the proposed MNEP algorithm is similar with that of the proposed MPME algorithm, but the computational cost of MNEP is much more expensive than that of MPME, as can be seen in Figure 4.9. Considering the previous discussions in Section 4.4-4.5, we can easily find that the proposed MEMP algorithm is the best algorithm for large scale physical field reconstruction.

\subsection{Concluding remarks}

Sensor placement for the physical field reconstruction problem is an interesting but challenging combinatorial problem. The optimal solution can be solved by the exhaustive search and branch-and-bound methods [62, 63], but the methods are both impractical due to the extremely expensive computational cost, especially for some large scale problems. Therefore, in the last decade, many works have focused on finding an effective suboptimal solution via computationally efficient algorithms. To the best of our knowledge, the proposed MPME algorithm is computationally one of the most efficient sensor placement algorithms.

Our proposed MNEP and MPME algorithms select the sensing locations one-byone. In this way, the minimum number of the required sensor nodes can be readily determined. Different with many popular methods, MNEP and MPME can guarantee that the dual observation matrix is well-conditioned when the number of sensor nodes is small and even near the dimension of the estimated vector.

The sufficient and necessary condition of meeting the requirement on WCEV is shown to be that the square summation of the projections of all selected observation vectors onto any non-trivial subspace of $\mathbb{R}^{n}$ is large enough. The proposed MPME algorithm determines each sensing location by maximizing the projection of its observation vector onto the subspace onto which the square summation of the projections of all selected observation vectors is minimum. 
We perform Monte-Carlo simulations to compare the MNEP and MPME algorithms with the convex relaxation method [64], SparSenSe [69], and FrameSense [65]. Based on the simulation results, we conclude that amongst the five methods:

- To meet the accuracy requirement, the solution of MPME requires the least number of sensor nodes;

- The MPME algorithm provides the best solution in the sense of minimum WCEV or minimum MSE, especially when the number of sensor nodes used is small;

- MNEP and MPME work well when the number of available sensor nodes is very limited, while the state-of-the-art cannot;

- For the general cases, the solution of the MPME without local optimization is even better than those of the state-of-the-art with local optimization.

In addition, the storage requirement of the MPME is much less than the state-ofthe-art. With a common compuate, current sensor placement algorithms cannot solve the large scale physical field reconstruction problems, like the thermal map reconstruction problem in Chapter 3, because of the high storage requirement. By contrast, the proposed MPME algorithm can immediately provide the solution in a laptop with 2GB RAM.

To encourage future works, we provide all the Matlab code used in this Chapter, which can be found from https://github.com/CJiang01/SensorPlacement . git. 



\section{Chapter 5}

\section{Conclusions and future work}

In this thesis, we address the question on how sparse sensor observations can be effectively used for physical field estimation by solving the following three problems:

1. Calibration of simulated physical fields with sparse sensor observations.

2. Reconstruction of physical fields from sparse sensor observations.

3. Sensor placement for the optimal physical field reconstruction.

The main idea to solve our question is the fusing of the sparse sensor observations with the physical fields obtained from CFD simulations. We apply the proposed methods to estimate the indoor thermal maps of an air-conditioned room. The results show that the proposed methods can be used for indoor thermal maps estimation.

A summary of the key results obtained from this thesis and some possible future works are given as follows:

1. Calibration of simulated physical fields: Sensor observations are much more accurate than the simulated physical fields. This work fuses one simulated physical field with sparse sensor observations to achieve a more accurate 
physical field. This is a very interesting problem but no reported publication has focused on this problem. We formulate this problem to be an optimization problem. The cost function consists of two terms. One term guarantees a good local adjustment of the simulated physical field. The other term transmits the adjustment from local regions around sensing locations to the global domain. With sparse sensor observations, we can obtain an explicit solution of the calibrated physical field. The proposed method can globally calibrate a simulated physical field with sparse sensor observations. The performance of the proposed method depends on three parameters of the cost function (i.e., the balance factor, the magnitude variance, and the distance variance), the number of sensor observations, and their sensing locations. We have qualitatively analyzed the relation between the three factors and the performance of the proposed method, but there are no quantitative results. The limitation of this work is that it can only be used for a given simulated physical field. If the input parameters of the CFD simulation (e.g., the heat sources) change, we need to rerun the CFD simulation and recalibrate the new simulated physical field. Future work can be developed from the following three aspects:

- A detail analysis of the relation between the three parameters with the performance of the proposed method to find the best solution. We have not provided quantitative guides on the parameters setting.

- Uncover the influence of the number of sensor observations and their locations on the calibration results. We have not discussed the influence of the observation number and their locations.

- The design of physical distance between sensors based on the given calibration performance is also an interesting research direction.

2. Reconstruction of physical fields: This work fuses a simulated physical field database with sparse sensor observations to directly estimate one physical field from sparse sensor observations. Even the environment (e.g., heat sources) around the physical field changes, which corresponds to the 
change of the input parameters for a CFD simulation, we can directly estimate the physical field of interest from sparse sensor observations without redoing the CFD simulation. This is the main difference from the calibration work. The sparse sensor observations refer to the observed input parameters of the CFD simulation and the partially observed physical field. Current methods separately use the observed parameters and the sparse observations of the physical field. No reported publication has fused both of them. In this work, we estimate the physical field in two stages. In the first stage, we estimate the PCA components of the physical field from a regression model with the observed input parameters. In the second stage, we correct the estimated physical field in the first stage using the sparse observations of the physical field. In some real applications, some input parameters can be easily obtained, e.g., we can easily obtain the ON/OFF state of heat sources without sensors. But the observations of physical fields may be expensive. Using the proposed method, and with the help of observed input parameters, we can save the number of sensor observations. With the cross validation for the regression model, we can find some PCA components that can be well-estimated in the first stage, which is not required to be corrected in the second stage. With smaller number of PCA components to be estimated, we can reduce the number of sensor observations. The example for the indoor thermal map estimation shows that the proposed TSPFR method outperforms current methods. We reduce the number of sensor observations from four to two but the estimation performance is still better than the current methods with four sensor observations. For this physical field reconstruction problem, the sensing locations are extremely important unless enough sensor observations are available. Future work can perhaps focus on the following three aspects:

- In this work, a physical field database is required. The samples (i.e., the physical fields) in the database are obtained from CFD simulations. Building such a database is tedious and expensive. For a particular 
application, is it possible to reduce the number of the samples in the database while maintaining the accuracy of estimated physical field? If yes, how to select the critical samples? We have not discussed the two problems.

- In addition, the simulated physical fields in the database have a gap with the real physical fields. Correspondingly, the PCA modes have a gap with the PCA modes of the real physical fields. In this work, we ignore the gap. However, when using the observations of a real physical field and the PCA modes of the simulated physical fields to reconstruct the physical field, the inaccurate PCA modes definitely have negative influence on the estimation results. It is of interest to quantitatively analyze the influence of the error of the PCA modes on the physical field reconstruction.

- In this work, we only consider the reconstruction of steady-state physical fields. The heat sources are assumed to be fixed. Sensor network can provide real-time observations. Is it possible to reconstruct dynamic physical fields with time-varying heat sources?

3. Sensor placement for physical field reconstruction: Sensor placement for physical field reconstruction is a challenging combinatorial problem. The aim of sensor placement design is to minimize the number of required sensor observations and guarantee that the estimation error meets the requirement. The exhaustive search and branch-and-bound methods can provide the optimal solution but the computation cost is extremely high. Currently, we cannot find the optimal solution for large scale problems. We perform Monte-Carlo simulations to compare the proposed MPME algorithm with the convex relaxation method, the SparSenSe method, and the FrameSense method. Based on the simulation results, we conclude that amongst the four methods:

- The MPME is computationally most efficient; 
- To meet the accuracy requirement, the solution of MPME requires the least number of sensor nodes;

- The MPME algorithm provides the best solution in the sense of minimum WCEV or minimum MSE, especially when the number of sensor nodes used is small;

- MNEP and MPME work well when the number of available sensor nodes is very limited, while the others cannot;

- For the general cases, the solution of the MPME without local optimization is even better than those of the state-of-the-art with local optimization.

If the norms of the rows of the signal representation matrix are equal, the signal representation matrix corresponds to a equal-norm frame. For the equal-norm frame case, the FrameSense method with local optimization slightly outperforms the proposed MPME with/without local optimization. However, the signal representation matrix consists of the dominant PCA modes whose rows' norm are usually different. Thus, the proposed MPME outperforms the FrameSense method for physical field reconstruction problem. In addition, The storage requirement of the MPME is much less than the state-of-the-art. In the example of indoor thermal map reconstruction, the convex relaxation method, the SparSenSe method, and the FrameSense method cannot work on a workstation with 16GB RAM due to the shortage of memory. The proposed MPME can immediately provide the solution in a laptop with $2 \mathrm{~GB}$ RAM.

Future work can be developed from the following two aspects:

- The solution of FrameSense is near-optimal because of the sub-modularity of the cost function. However, the proposed MPME algorithm still outperforms FrameSense in the proposed four examples. It is interesting to explore the reason why MPME outperforms the near-optimal solution 
from a theoretical perspective. Is it possible to find a error bound for the proposed MPME algorithm?

- In this work, we assume that the noise of sensor observations follows the independent and identically normal distribution. It is interesting to solve the sensor placement problem using greedy method for the case of correlated measurement noise. 


\section{Appendix A}

\section{Proof of Theorem 2}

Proof. The spectrum decomposition of $\boldsymbol{\Psi}_{k}$ is

$$
\boldsymbol{\Psi}_{k}=\boldsymbol{\Phi}_{k}^{\mathrm{T}} \boldsymbol{\Phi}_{k}=\mathbf{U}^{(k)} \boldsymbol{\Lambda}_{k}\left(\mathbf{U}^{(k)}\right)^{\mathrm{T}}
$$

where $\mathbf{U}^{(k)}=\left[\mathbf{u}_{1}^{(k)}, \mathbf{u}_{2}^{(k)}, \ldots, \mathbf{u}_{n}^{(k)}\right]$ is an orthonormal matrix, and $\boldsymbol{\Lambda}_{k}$ is a diagonal matrix whose diagonal entry $\boldsymbol{\Lambda}_{i i}^{(k)}=\lambda_{i}^{(k)}$. Then, we can obtain

$$
\boldsymbol{\Lambda}_{k}=\left(\mathbf{U}^{(k)}\right)^{\mathrm{T}} \boldsymbol{\Phi}_{k}^{\mathrm{T}} \boldsymbol{\Phi}_{k} \mathbf{U}^{(k)}
$$

from which (4.14) can be directly found. 



\section{Appendix B}

\section{Proof of Theorem 3}

To proof Theorem 3, we need the following lemma.

Lemma 1 (Courant-Fischer Minimax Theorem). If $\mathbf{A} \in \mathbb{R}^{n \times n}$ is symmetric, then for $i=1: n$,

$$
\lambda_{i}(\mathbf{A})=\max _{\operatorname{dim}(\mathbb{U})=i} \min _{\mathbf{0} \neq \mathbf{x} \in \mathbb{U}} \frac{\mathbf{x}^{\mathrm{T}} \mathbf{A} \mathbf{x}}{\mathbf{x}^{\mathrm{T}} \mathbf{x}}
$$

Proof. See the proof of Theorem 8.1.2 in [83].

Next, we prove Theorem 3.

Proof. Since $\mathbf{x}^{\mathrm{T}} \boldsymbol{\Psi}_{k} \mathbf{x}=\mathbf{x}^{\mathrm{T}} \boldsymbol{\Phi}_{k}^{\mathrm{T}} \boldsymbol{\Phi}_{k} \mathbf{x}=\left\|\boldsymbol{\Phi}_{k} \mathbf{x}\right\|_{2}^{2}$, from Lemma 1 we have

$$
\lambda_{n}^{(k)}=\min _{\|\mathbf{x}\|_{2}=1} \mathbf{x}^{\mathrm{T}} \mathbf{\Psi}_{k} \mathbf{x}=\min _{\|\mathbf{x}\|_{2}=1}\left\|\mathbf{\Phi}_{k} \mathbf{x}\right\|_{2}^{2}
$$

Then, from (4.14) and (B.2), we can obtain (4.15).

Next, we show the sufficient and necessary condition of $\lambda_{n}^{(k)}>\gamma$ is that for any nonzero normalized vector $\mathbf{x} \in \mathbb{R}^{n},\left\|\Phi_{k} \mathbf{x}\right\|_{2}^{2}>\gamma$.

Sufficiency: Considering (4.14), if $\left\|\boldsymbol{\Phi}_{k} \mathbf{x}\right\|_{2}^{2}>\gamma$ for any nonzero normalized vector $\mathbf{x} \in \mathbb{R}^{n}$, we can obtain $\lambda_{n}^{(k)}>\gamma$. 
Necessity: Since $\lambda_{n}^{(k)}>\gamma$, considering (B.2) we conclude that $\left\|\Phi_{k} \mathbf{x}\right\|_{2}^{2}>\gamma$ for any nonzero normalized vector $\mathbf{x} \in \mathbb{R}^{n}$. 


\section{Appendix $\mathrm{C}$}

\section{Proof of Theorem 4}

Considering (4.17) and (A.1), we can obtain another description of $\boldsymbol{\Psi}_{k}$, i.e.

$$
\boldsymbol{\Psi}_{k}=\boldsymbol{\Phi}_{k-1}^{\mathrm{T}} \boldsymbol{\Phi}_{k-1}+\boldsymbol{\varphi}_{s_{k}} \boldsymbol{\varphi}_{s_{k}}^{\mathrm{T}}=\mathbf{U}^{(k-1)}\left(\boldsymbol{\Lambda}_{k-1}+\mathbf{z z}^{\mathrm{T}}\right)\left(\mathbf{U}^{(k-1)}\right)^{\mathrm{T}}
$$

Let

$$
\hat{\Lambda}_{k}=\boldsymbol{\Lambda}_{k-1}+\mathbf{z z}^{\mathrm{T}}
$$

From (A.1) and (C.1), we find that $\boldsymbol{\Psi}_{k}, \boldsymbol{\Lambda}_{k}$, and $\hat{\boldsymbol{\Lambda}}_{k}$ are mutually similar, which implies that they share the same eigenvalues.

To proof Theorem 4, we need the following four lemmas.

Lemma 2. If $z_{i}=0, \lambda_{i}^{(k-1)}$ is an eigenvalue of $\hat{\boldsymbol{\Lambda}}_{k}$, and the corresponding eigenvector is $\mathbf{e}_{i}$

Proof. As $z_{i}=0$, we can obtain

$$
\hat{\Lambda}_{k} \mathbf{e}_{i}=\boldsymbol{\Lambda}_{k-1} \mathbf{e}_{i}+\mathbf{z z}^{\mathrm{T}} \mathbf{e}_{i}=\lambda_{i}^{(k-1)} \mathbf{e}_{i}
$$

It is clear that $\lambda_{i}^{(k-1)}$ is an eigenvalue of $\hat{\boldsymbol{\Lambda}}_{k}$ and $\mathbf{e}_{i}$ is the corresponding eigenvector. 
Lemma 3. For all $i \in \mathcal{I}=\left\{i \mid 1 \leq i \leq n, z_{i} \neq 0\right\}$, denote by $\mathbf{v}_{i}$ the eigenvector of $\hat{\Lambda}_{k}$ associated with $\lambda_{i}^{(k)}$. For all $j \in \mathcal{J}=\left\{j \mid 1 \leq j \leq n, z_{j}=0\right\}$, removing the $j$-th row and $j$-th column of $\boldsymbol{\Lambda}_{k-1}$ and $\hat{\boldsymbol{\Lambda}}_{k}$ yields $\tilde{\boldsymbol{\Lambda}}_{k-1}$ and $\tilde{\boldsymbol{\Lambda}}_{k}$, respectively, and removing the $j$-th entry of $\mathbf{v}_{i}$ and $\mathbf{z}$ yields $\tilde{\mathbf{v}}_{i}$ and $\tilde{\mathbf{z}}$, respectively. Then,

$$
\tilde{\boldsymbol{\Lambda}}_{k} \tilde{\mathbf{v}}_{i}=\tilde{\Lambda}_{k-1} \tilde{\mathbf{v}}_{i}+\tilde{\mathbf{z}} \tilde{\mathbf{z}}^{\mathrm{T}} \tilde{\mathbf{v}}_{i}=\lambda_{i}^{(k)} \tilde{\mathbf{v}}_{i}
$$

Proof. For all $j \in \mathcal{J}$, removing the $j$-th row of the following equation directly yields (C.2).

$$
\boldsymbol{\Lambda}_{k} \mathbf{v}_{i}=\boldsymbol{\Lambda}_{k-1} \mathbf{v}_{i}+\mathbf{z}\left(\mathbf{z}^{\mathrm{T}} \mathbf{v}_{i}\right)=\lambda_{i}^{(k)} \mathbf{v}_{i}
$$

Lemma 4. If $\lambda_{i}^{(k-1)}=\lambda_{i+\mu_{i}-1}^{(k-1)}$ is an eigenvalue of $\boldsymbol{\Lambda}_{k-1}$ with multiplicity $\mu_{i}$ and $\sum_{j=i}^{i+\mu_{i}-1} z_{j}^{2} \neq 0$, then $\lambda_{i}^{(k-1)}$ is an eigenvalue of $\hat{\Lambda}_{k}$ with multiplicity $\mu_{i}-1$.

Proof. Since $\sum_{j=i}^{i+\mu_{i}-1} z_{j}^{2} \neq 0$, we can obtain

$$
\begin{aligned}
\operatorname{rank}\left(\hat{\Lambda}_{k}-\lambda_{i}^{(k-1)} \mathbf{I}\right) & =\operatorname{dim} \operatorname{span}\left(\hat{\Lambda}_{k}-\lambda_{i}^{(k-1)} \mathbf{I}\right) \\
& =\operatorname{dim} \operatorname{span}\left(\left[\boldsymbol{\Lambda}_{k-1}-\lambda_{i}^{(k-1)} \mathbf{I} \quad \mathbf{z}\right]\right) \\
& =n-\mu_{i}+1
\end{aligned}
$$

where for any matrix $\mathbf{A}$, $\operatorname{dim} \operatorname{span}(\mathbf{A})$ represents the dimension of a linear space spanned by all the column vectors of $\mathbf{A}$. Therefore, $\lambda_{i}^{(k-1)}$ is an eigenvalue of $\hat{\boldsymbol{\Lambda}}_{k}$ with multiplicity $\mu_{i}-1$.

Lemma 5. If $\lambda_{n}^{(k-1)}$ is a multiple eigenvalue of $\boldsymbol{\Lambda}_{k-1}$ with multiplicity $\mu_{n}$ and $\sum_{j=n-\mu_{n}+1}^{n} z_{j}^{2} \neq 0$, then $\lambda_{n-\mu_{n}+1}^{(k)} \neq \lambda_{i}^{(k-1)}$ for all $i$ satisfying $z_{i} \neq 0$.

Proof. It follows from Theorem 1 that

$$
\lambda_{i}^{(k-1)} \leq \lambda_{i}^{(k)} \leq \lambda_{i-1}^{(k-1)} \text { for all } 1<i \leq n
$$


Hence,

$$
\lambda_{n-\mu_{n}+1}^{(k-1)} \leq \lambda_{n-\mu_{n}+1}^{(k)} \leq \lambda_{n-\mu_{n}}^{(k-1)}
$$

Then, considering Lemma 4, we can obtain

$$
\lambda_{n-\mu_{n}+1}^{(k)}>\lambda_{n-\mu_{n}+1}^{(k-1)}=\lambda_{n}^{(k-1)}=\lambda_{n-\mu_{n}+2}^{(k)}=\lambda_{n}^{(k)}
$$

Denote the multiplicity of $\lambda_{n-\mu_{n}}^{(k-1)}$ w.r.t. $\boldsymbol{\Lambda}_{k-1}$ by $\mu$, where $\mu \geq 1$. Hence,

$$
\lambda_{n-\mu_{n}-\mu}^{(k-1)}>\lambda_{n-\mu_{n}-\mu+1}^{(k-1)}=\lambda_{n-\mu_{n}-\mu+2}^{(k-1)}=\ldots=\lambda_{n-\mu_{n}}^{(k-1)}
$$

If $\sum_{i=n-\mu_{n}-\mu+1}^{n-\mu_{n}} z_{i}^{2} \neq 0$, considering Lemma $4, \lambda_{n-\mu_{n}}^{(k-1)}$ is an eigenvalue of $\hat{\boldsymbol{\Lambda}}_{k}$ with multiplicity $\mu-1$. Therefore, if

$$
\lambda_{n-\mu_{n}}^{(k-1)}=\lambda_{n-\mu_{n}+1}^{(k)}
$$

the multiplicity of $\lambda_{n-\mu_{n}+1}^{(k)}$ w.r.t. $\hat{\Lambda}_{k}$ is $\mu-1$ and

$$
\lambda_{n-\mu_{n}+1}^{(k)}=\lambda_{n-\mu_{n}}^{(k)}=\ldots=\lambda_{n-\mu_{n}-\mu+3}^{(k)}<\lambda_{n-\mu_{n}-\mu+2}^{(k)}
$$

From (C.6)-(C.8), we can obtain $\lambda_{n-\mu_{n}-\mu+1}^{(k-1)}<\lambda_{n-\mu_{n}-\mu+2}^{(k)}$, which obviously contradict with (C.3). Hence, $\lambda_{n-\mu_{n}+1}^{(k)} \neq \lambda_{n-\mu_{n}}^{(k-1)}$.

Consequently, if $\sum_{i=n-\mu_{n}-\mu+1}^{n-\mu_{n}} z_{i}^{2} \neq 0$, considering (C.4) and (C.5) we can obtain

$$
\lambda_{n-\mu_{n}+1}^{(k-1)}<\lambda_{n-\mu_{n}+1}^{(k)}<\lambda_{n-\mu_{n}}^{(k-1)} \leq \lambda_{j}^{(k-1)}, \quad j<n-\mu_{n}
$$

else $\left(\sum_{i=n-\mu_{n}-\mu+1}^{n-\mu_{n}} z_{i}^{2}=0\right)$

$$
\lambda_{n-\mu_{n}+1}^{(k-1)}<\lambda_{n-\mu_{n}+1}^{(k)} \leq \lambda_{n-\mu_{n}}^{(k-1)} \leq \lambda_{j}^{(k-1)}, \quad j<n-\mu_{n}
$$

which implies this lemma. 
Next, leveraging the four Lemmas, we are ready to prove Theorem 4 .

Proof. Let $\lambda_{n}^{(k-1)}$ be the minimum eigenvalue of $\boldsymbol{\Psi}_{k-1}\left(\boldsymbol{\Lambda}_{k-1}\right)$ with multiplicity $\mu_{n}(\geq 1)$. If $k<n, \lambda_{n}^{(k-1)}=0$ and $\mu_{n}=n-k+1$. From Lemma 4 , we can directly obtain (4.18b) and (4.19b).

If $\sum_{i=n-\mu_{n}+1}^{n} z_{i}^{2}=0$, it is obvious that $\zeta_{k}=0$. According to Lemma 2, we can easily obtain (4.18a) and (4.19a).

If $\sum_{i=n-\mu_{n}+1}^{n} z_{i}^{2} \neq 0$, according to Lemma 3, we can obtain

$$
\left(\tilde{\boldsymbol{\Lambda}}_{k-1}-\lambda_{n-\mu_{n}+1}^{(k)} \mathbf{I}\right) \tilde{\mathbf{v}}_{n-\mu_{n}+1}+\tilde{\mathbf{z}}\left(\tilde{\mathbf{z}}^{\mathrm{T}} \tilde{\mathbf{v}}_{n-\mu_{n}+1}\right)=0
$$

Lemma 5 can guarantee that $\tilde{\boldsymbol{\Lambda}}_{k-1}-\lambda_{n-\mu_{n}+1}^{(k)} \mathbf{I}$ is nonsingular, and therefore from (C.9), we find that $\tilde{\mathbf{z}}^{\mathrm{T}} \tilde{\mathbf{v}}_{n-\mu_{n}+1} \neq 0$. Then, left multiplying $\tilde{\mathbf{z}}^{\mathrm{T}}\left(\tilde{\boldsymbol{\Lambda}}_{k-1}-\lambda_{n-\mu_{n}+1}^{(k)} \mathbf{I}\right)^{-1}$ to both sides of (C.9) yields

$$
\tilde{\mathbf{z}}^{\mathrm{T}} \tilde{\mathbf{v}}_{n-\mu_{n}+1}\left(1+\tilde{\mathbf{z}}^{\mathrm{T}}\left(\tilde{\boldsymbol{\Lambda}}_{k-1}-\lambda_{n-\mu_{n}+1}^{(k)} \mathbf{I}\right)^{-1} \tilde{\mathbf{z}}\right)=0
$$

Hence,

$$
1+\tilde{\mathbf{z}}^{\mathrm{T}}\left(\tilde{\boldsymbol{\Lambda}}_{k-1}-\lambda_{n-\mu_{n}+1}^{(k)} \mathbf{I}\right)^{-1} \tilde{\mathbf{z}}=0
$$

from which we can directly obtain (4.19a). If $k \leq n, \lambda_{k}^{(k-1)}=0$ and $\mu_{n}=n-k+1$, substituting them into (4.19a) yields (4.18a).

It is clear that Equation (4.19a) is a general description of (4.18a). From (4.19a), we can obtain

$$
\zeta_{k}=\lambda_{n-\mu_{n}+1}^{(k)}-\lambda_{n-\mu_{n}+1}^{(k-1)}+\sum_{i=1, z_{i} \neq 0}^{n-\mu_{n}} \frac{\left(\lambda_{n-\mu_{n}+1}^{(k)}-\lambda_{n-\mu_{n}+1}^{(k-1)}\right) z_{i}^{2}}{\lambda_{i}^{(k-1)}-\lambda_{n-\mu_{n}+1}^{(k)}}
$$

Taking the derivative of $\zeta_{k}$ w.r.t. $\lambda_{n-\mu_{n}+1}^{(k)}$, we can obtain that

$$
\frac{\mathrm{d} \zeta_{k}}{\mathrm{~d} \lambda_{n-\mu_{n}+1}^{(k)}}=1+\sum_{i=1, z_{i} \neq 0}^{n-\mu_{n}} \frac{\left(\lambda_{i}^{(k-1)}-\lambda_{n-\mu_{n}+1}^{(k-1)}\right) z_{i}^{2}}{\left(\lambda_{i}^{(k-1)}-\lambda_{n-\mu_{n}+1}^{(k)}\right)^{2}}
$$


Since $\lambda_{i}^{(k-1)} \geq \lambda_{n-\mu_{n}+1}^{(k-1)}$ for all $i \leq n-\mu_{n}$, it is obvious that $\mathrm{d} \zeta_{k} / \mathrm{d} \lambda_{n}^{(k)} \geq 1$. Therefore, $0<\mathrm{d} \lambda_{n-\mu_{n}+1}^{(k)} / \mathrm{d} \zeta_{k} \leq 1$, which implies that $\lambda_{n-\mu_{n}+1}^{(k)}$ is monotonically strictly increasing w.r.t. $\zeta_{k}$, i.e.

$$
\zeta_{k} \leftrightarrow \lambda_{n-\mu_{n}+1}^{(k)}
$$

where ' $a \leftrightarrow b$ ' means that $b$ is monotonically increasing w.r.t. $a$.

Taking derivative of both sides of (C.10) w.r.t. $\lambda_{n-\mu_{n}}^{(k-1)}$ and with some operations, we can obtain

$$
\frac{\mathrm{d} \lambda_{n-\mu_{n}+1}^{(k)}}{\mathrm{d} \lambda_{n-\mu_{n}}^{(k-1)}}=\frac{\lambda_{n-\mu_{n}+1}^{(k)} z_{n-\mu_{n}}^{2}}{\left(\lambda_{n-\mu_{n}}^{(k-1)}-\lambda_{n-\mu_{n}+1}^{(k)}\right)^{2}(1+x)}
$$

where

$$
x=\sum_{i=1, z_{i} \neq 0}^{n-\mu_{n}} \frac{\lambda_{i}^{(k-1)} z_{i}^{2}}{\left(\lambda_{i}^{(k-1)}-\lambda_{n-\mu_{n}+1}^{(k)}\right)^{2}}
$$

Since $\lambda_{n-\mu_{n}+1}^{(k)}>0$ and $\lambda_{i}^{(k-1)}>0$ for all $i \leq n-\mu_{n}$, we can obtain that $\mathrm{d} \lambda_{n-\mu_{n}+1}^{(k)} / \mathrm{d} \lambda_{n-\mu_{n}}^{(k-1)} \geq 0$, which implies that $\lambda_{n-\mu_{n}+1}^{(k)}$ is monotonically increasing w.r.t. $\lambda_{n-\mu_{n}}^{(k-1)}$, i.e.

$$
\lambda_{n-\mu_{n}}^{(k-1)} \leftrightarrow \lambda_{n-\mu_{n}+1}^{(k)}
$$

If $k \leq n$, we have $\mu_{n}=n-k+1$ and therefore, $\lambda_{k}^{(k)}$ is monotonically increasing w.r.t. $\lambda_{k-1}^{(k-1)}$ for all $k \leq n$, i.e.

$$
\lambda_{k-1}^{(k-1)} \leftrightarrow \lambda_{k}^{(k)}
$$

Taking the derivative of both sides of (C.10) w.r.t. $\lambda_{n-\mu_{n}+1}^{(k-1)}$ and with some operations, we can obtain that

$$
\frac{\mathrm{d} \lambda_{n-\mu_{n}+1}^{(k)}}{\mathrm{d} \lambda_{n-\mu_{n}+1}^{(k-1)}}=\frac{1+\sum_{i=1, z_{i} \neq 0}^{n-\mu_{n}} \frac{z_{i}^{2}}{1+\sum_{i=1, z_{i} \neq 0}^{n-\mu_{n}} \frac{\left(\lambda_{i}^{(k-1)}-\lambda_{n-\mu_{n+1}}^{(k-1)} z_{i}^{2}\right.}{\left(\lambda_{i}^{(k-1)}-\lambda_{n-\mu_{n}+1}^{(k)}\right)^{2}}}}{1(k)}
$$

Since $\lambda_{i}^{(k-1)} \geq \lambda_{n-\mu_{n}+1}^{(k-1)}$ for all $i \leq n-\mu_{n}$, we can obtain that $\mathrm{d} \lambda_{n-\mu_{n}+1}^{(k)} / \mathrm{d} \lambda_{n-\mu_{n}+1}^{(k-1)}>$ 0 , which implies that $\lambda_{n-\mu_{n}+1}^{(k)}$ is monotonically strictly increasing w.r.t. $\lambda_{n-\mu_{n}+1}^{(k-1)}$; 
therefore,

$$
\lambda_{n-\mu_{n}+1}^{(k-1)} \leftrightarrow \lambda_{n-\mu_{n}+1}^{(k)}
$$

For $n \leq k \leq M$, generally $\mu_{n}=1$ and from (C.11) and (C.14) we can obtain that

$$
\zeta_{k} \leftrightarrow \lambda_{n}^{(k)} \leftrightarrow \lambda_{n}^{(k+1)} \leftrightarrow \ldots \leftrightarrow \lambda_{n}^{(M)}
$$

If $\mu_{n}>1$, from (C.11) and (C.12) we can obtain that

$$
\zeta_{k} \leftrightarrow \lambda_{n-\mu_{n}+1}^{(k)} \leftrightarrow \lambda_{n-\mu_{n}+2}^{(k+1)} \leftrightarrow \ldots \leftrightarrow \lambda_{n}^{\left(k+\mu_{n}-1\right)}
$$

Since $\lambda_{n}^{\left(k+\mu_{n}-1\right)}$ is a simple eigenvalue, considering (C.14) and (C.16) we can find

$$
\zeta_{k} \leftrightarrow \lambda_{n}^{\left(k+\mu_{n}-1\right)} \leftrightarrow \lambda_{n}^{\left(k+\mu_{n}\right)} \leftrightarrow \ldots \leftrightarrow \lambda_{n}^{(M)}
$$

From (C.15)-(C.17), we can obtain that

$$
\lambda_{n}^{(n)} \leftrightarrow \lambda_{n}^{(M)}
$$

For $k<n$, the multiplicity of $\lambda_{n}^{(k-1)}$ w.r.t. $\Psi_{k-1}$ is $n-k+1$, i.e. $\mu_{n}=n-k+1$. Then, considering (C.11), (C.13) and (C.18), we can obtain that

$$
\zeta_{k} \leftrightarrow \lambda_{k}^{(k)} \leftrightarrow \lambda_{k+1}^{(k+1)} \leftrightarrow \ldots \leftrightarrow \lambda_{n}^{(n)} \leftrightarrow \lambda_{n}^{(M)}
$$

In summary, from (C.15), (C.17) and (C.19), we can conclude that for any $M \geq n$, $\lambda_{n}^{(M)}$ is monotonically increasing w.r.t. $\zeta_{k}$ for all $k \leq M$. 


\section{Author's Publications}

\section{Journal Articles:}

- Chaoyang Jiang, Yeng Chai Soh and Hua Li, "Two stage physical field estimation from sparse sensor observations with a case study for indoor thermal map," Energy and Buildings, 2017 (accepted).

- Chaoyang Jiang, Yeng Chai Soh, Hua Li, Mustafa K. Masood, Zhe Wei, Xiaoli Zhou, and Deqing Zhai, "CFD results calibration from sparse sensor observations with a case study for indoor thermal map," Building and Environment, vol. 117, pp. 166-177, 2017.

- Chaoyang Jiang, Yeng Chai Soh, and Hua Li, "Sensor placement by maximal projection on minimum eigenspace for linear inverse problem," IEEE Transactions on Signal Processing, vol. 64, no. 21, pp. 5595-5610, 2016.

- Chaoyang Jiang, Mustafa K. Masood, Yeng Chai Soh, and Hua Li, "Indoor occupancy estimation from carbon dioxide concentration," Energy and Buildings, vol. 131, pp. 132-141, 2016.

- Chaoyang Jiang, Yeng Chai Soh and Hua Li, "Sensor and CFD data fusion for airflow field estimation," Applied Thermal Engineering, vol. 92, pp. 149$161,2016$.

\section{Conference Proceedings:}

- Chaoyang Jiang, Yeng Chai Soh, Hua Li, and Hongming Zhou "Physical field estimation from CFD database and sparse sensor observations," in IEEE International Conference on Automation Science and Engineering (CASE), 2015. 



\section{Bibliography}

[1] C. Allocca, Q. Chen, and L. R. Glicksman, "Design analysis of single-sided natural ventilation," Energy and Buildings, vol. 35, no. 8, pp. 785-795, 2003.

[2] A. M. Omer, "Renewable building energy systems and passive human comfort solutions," Renewable and Sustainable Energy Reviews, vol. 12, no. 6, pp. 1562-1587, 2008.

[3] K. Chua, S. Chou, W. Yang, and J. Yan, "Achieving better energy-efficient air conditioning-a review of technologies and strategies," Applied Energy, vol. 104, pp. 87-104, 2013.

[4] E. E. Singappore, "www.e2singapore.gov.sg/docs/e2_singapore.pdf." 2005.

[5] H. Bhagwat, A. K. Singh, R. Jayaprakash, A. Sivasubramaniam et al., "Method and system for real time monitoring, prediction, analysis and display of temperatures for effective thermal management in a data center," Mar. 22 2016, uS Patent 9,295,183.

[6] Y. Joshi and P. Kumar, Energy efficient thermal management of data centers. Springer Science \& Business Media, 2012.

[7] E. Oró, V. Depoorter, A. Garcia, and J. Salom, "Energy efficiency and renewable energy integration in data centres. strategies and modelling review," Renewable and Sustainable Energy Reviews, vol. 42, pp. 429-445, 2015.

[8] J. Ni and X. Bai, "A review of air conditioning energy performance in data centers," Renewable and Sustainable Energy Reviews, vol. 67, pp. 625-640, 2017. 
[9] K. Cheong, E. Djunaedy, Y. Chua, K. Tham, S. Sekhar, N. Wong, and M. Ullah, "Thermal comfort study of an air-conditioned lecture theatre in the tropics," Building and Environment, vol. 38, no. 1, pp. 63-73, 2003.

[10] N. Gao, J. Niu, and H. Zhang, "Coupling cfd and human body thermoregulation model for the assessment of personalized ventilation," HVACERR Research, vol. 12, no. 3, pp. 497-518, 2006.

[11] T. Catalina, J. Virgone, and F. Kuznik, "Evaluation of thermal comfort using combined CFD and experimentation study in a test room equipped with a cooling ceiling," Building and Environment, vol. 44, no. 8, pp. 1740-1750, 2009.

[12] H. Zhang, L. Dai, G. Xu, Y. Li, W. Chen, and W.-Q. Tao, "Studies of air-flow and temperature fields inside a passenger compartment for improving thermal comfort and saving energy. part i: Test/numerical model and validation," Applied Thermal Engineering, vol. 29, no. 10, pp. 2022-2027, 2009.

[13] H. Zhang, L. Dai, G. Xu, Y. Li, W. Chen, and W. Tao, "Studies of air-flow and temperature fields inside a passenger compartment for improving thermal comfort and saving energy. part ii: Simulation results and discussion," Applied Thermal Engineering, vol. 29, no. 10, pp. 2028-2036, 2009.

[14] R. Dear, T. Akimoto, E. Arens, G. Brager, C. Candido, K. Cheong, B. Li, N. Nishihara, S. Sekhar, S. Tanabe et al., "Progress in thermal comfort research over the last twenty years," Indoor Air, vol. 23, no. 6, pp. 442-461, 2013.

[15] G. Stavrakakis, P. Zervas, H. Sarimveis, and N. Markatos, "Development of a computational tool to quantify architectural-design effects on thermal comfort in naturally ventilated rural houses," Building and Environment, vol. 45, no. 1, pp. 65-80, 2010. 
[16] G. Stavrakakis, M. Koukou, M. G. Vrachopoulos, and N. Markatos, "Natural cross-ventilation in buildings: building-scale experiments, numerical simulation and thermal comfort evaluation," Energy and Buildings, vol. 40, no. 9, pp. 1666-1681, 2008.

[17] G. Stavrakakis, P. Zervas, H. Sarimveis, and N. Markatos, "Optimization of window-openings design for thermal comfort in naturally ventilated buildings," Applied Mathematical Modelling, vol. 36, no. 1, pp. 193-211, 2012.

[18] J. Liang and R. Du, "Design of intelligent comfort control system with human learning and minimum power control strategies," Energy Conversion and Management, vol. 49, no. 4, pp. 517-528, 2008.

[19] R. Z. Freire, G. H. Oliveira, and N. Mendes, "Predictive controllers for thermal comfort optimization and energy savings," Energy and buildings, vol. 40, no. 7, pp. 1353-1365, 2008.

[20] F. Calvino, M. La Gennusa, M. Morale, G. Rizzo, and G. Scaccianoce, "Comparing different control strategies for indoor thermal comfort aimed at the evaluation of the energy cost of quality of building," Applied Thermal Engineering, vol. 30, no. 16, pp. 2386-2395, 2010.

[21] Y. Yao, K. Yang, M. Huang, and L. Wang, "A state-space model for dynamic response of indoor air temperature and humidity," Building and Environment, vol. 64, pp. 26-37, 2013.

[22] L. J. Lo and A. Novoselac, "Localized air-conditioning with occupancy control in an open office," Energy and Buildings, vol. 42, no. 7, pp. 1120-1128, 2010 .

[23] J.-H. Yang, T. Kim, and C. H. Cheong, "Supply airflow control algorithm of a floor-standing room air-conditioner to achieve thermal comfort for residential housing in summer," Building and Environment, vol. 59, pp. 227-238, 2013. 
[24] M. Wang, E. Wolfe, D. Ghosh, J. Bozeman, K.-h. Chen, T. Han, H. Zhang, and E. Arens, "Localized cooling for human comfort," SAE Technical Paper, Tech. Rep., 2014.

[25] H. Zhou, Y. C. Soh, and X. Wu, "Integrated analysis of CFD data with kmeans clustering algorithm and extreme learning machine for localized hvac control," Applied Thermal Engineering, vol. 76, pp. 98-104, 2015.

[26] T. Z. Desta, K. Janssens, A. Van Brecht, J. Meyers, M. Baelmans, and D. Berckmans, "CFD for model-based controller development," Building and Environment, vol. 39, no. 6, pp. 621-633, 2004.

[27] K. Li, H. Su, J. Chu, and C. Xu, "A fast-POD model for simulation and control of indoor thermal environment of buildings," Building and Environment, vol. 60, pp. 150-157, 2013.

[28] B. Gowreesunker and S. Tassou, "Effectiveness of CFD simulation for the performance prediction of phase change building boards in the thermal environment control of indoor spaces," Building and Environment, vol. 59, pp. 612-625, 2013.

[29] P. V. Nielsen, "Fifty years of CFD for room air distribution," Building and Environment, vol. 91, pp. 78-90, 2015.

[30] J. D. Anderson et al., Computational fluid dynamics. Springer, 1995, vol. 206.

[31] J. Srebric, V. Vukovic, G. He, and X. Yang, "CFD boundary conditions for contaminant dispersion, heat transfer and airflow simulations around human occupants in indoor environments," Building and Environment, vol. 43, no. 3, pp. 294-303, 2008.

[32] M. Hajdukiewicz, M. Geron, and M. M. Keane, "Formal calibration methodology for CFD models of naturally ventilated indoor environments," Building and Environment, vol. 59, pp. 290-302, 2013. 
[33] O. T. Kajero, R. B. Thorpe, T. Chen, B. Wang, and Y. Yao, "Kriging metamodel assisted calibration of computational fluid dynamics models," AIChE Journal, 2016.

[34] S. Guillas, N. Glover, and L. Malki-Epshtein, "Bayesian calibration of the constants of the $\mathrm{k}-\varepsilon$ turbulence model for a CFD model of street canyon flow," Computer Methods in Applied Mechanics and Engineering, vol. 279, pp. 536-553, 2014.

[35] A. Kylili, P. A. Fokaides, P. Christou, and S. A. Kalogirou, "Infrared thermography (irt) applications for building diagnostics: A review," Applied Energy, vol. 134, pp. 531-549, 2014.

[36] C. Lee and H. Yang, "A context-awareness system that uses a thermographic camera to monitor energy waste in buildings," Energy and Buildings, vol. 135, pp. 148-155, 2017.

[37] K. Kurec, J. Piechna, and K. Gumowski, "Investigations on unsteady flow within a stationary passage of a pressure wave exchanger, by means of PIV measurements and CFD calculations," Applied Thermal Engineering, vol. 112, pp. 610-620, 2017.

[38] S. Alimohammadi, E. Fanning, T. Persoons, and D. B. Murray, "Characterization of flow vectoring phenomenon in adjacent synthetic jets using CFD and PIV," Computers \&G Fluids, vol. 140, pp. 232-246, 2016.

[39] Q. Tang, S. K. S. Gupta, and G. Varsamopoulos, "Energy-efficient thermalaware task scheduling for homogeneous high-performance computing data centers: A cyber-physical approach," IEEE Transactions on Parallel and Distributed Systems, vol. 19, no. 11, pp. 1458-1472, 2008.

[40] R. K. Sharma, C. E. Bash, C. D. Patel, R. J. Friedrich, and J. S. Chase, "Balance of power: Dynamic thermal management for internet data centers," IEEE Internet Computing, vol. 9, no. 1, pp. 42-49, 2005. 
[41] J. D. Moore, J. S. Chase, P. Ranganathan, and R. K. Sharma, "Making scheduling" cool": Temperature-aware workload placement in data centers." in General Track, USENIX Annual Technical Conference O, 2005, pp. 61-75.

[42] M. Hajdukiewicz, M. Geron, and M. M. Keane, "Calibrated CFD simulation to evaluate thermal comfort in a highly-glazed naturally ventilated room," Building and Environment, vol. 70, pp. 73-89, 2013.

[43] A. Stamou and I. Katsiris, "Verification of a CFD model for indoor airflow and heat transfer," Building and Environment, vol. 41, no. 9, pp. 1171-1181, 2006.

[44] C. J. Roy and W. L. Oberkampf, "A comprehensive framework for verification, validation, and uncertainty quantification in scientific computing," Computer Methods in Applied Mechanics and Engineering, vol. 200, no. 25, pp. 2131-2144, 2011.

[45] R. Ramponi and B. Blocken, "CFD simulation of cross-ventilation for a generic isolated building: impact of computational parameters," Building and Environment, vol. 53, pp. 34-48, 2012.

[46] W.-H. Chiang, C.-Y. Wang, and J.-S. Huang, "Evaluation of cooling ceiling and mechanical ventilation systems on thermal comfort using CFD study in an office for subtropical region," Building and Environment, vol. 48, pp. 113-127, 2012.

[47] T. Van Waterschoot and G. Leus, "Distributed estimation of static fields in wireless sensor networks using the finite element method," in Acoustics, Speech and Signal Processing (ICASSP), IEEE International Conference on, 2012, pp. 2853-2856.

[48] B. Peherstorfer, and K. Willcox, "Dynamic data-driven reduced-order models," Computer Methods in Applied Mechanics and Engineering, vol. 291, pp. 21-41, 2015. 
[49] B. Peherstorfer, D. Butnaru, K. Willcox, and H.-J. Bungartz, "Localized discrete empirical interpolation method," SIAM Journal on Scientific Computing, vol. 36, no. 1, pp. A168-A192, 2014.

[50] Y. Liang, H. Lee, S. Lim, W. Lin, K. Lee, and C. Wu, "Proper orthogonal decomposition and its applications - part i: Theory," Journal of Sound and Vibration, vol. 252, no. 3, pp. 527-544, 2002.

[51] P. Holmes, Turbulence, coherent structures, dynamical systems and symmetry. Cambridge University Press, 2012.

[52] D. Eberly. (2015) Thin plate splines. [Online]. Available: http://www.geometrictools.com/Documentation/ThinPlateSplines.pdf

[53] H. V. Ly and H. T. Tran, "Modeling and control of physical processes using proper orthogonal decomposition," Mathematical and computer modelling, vol. 33, no. 1, pp. 223-236, 2001.

[54] T. Bui-Thanh, M. Damodaran, and K. Willcox, "Proper orthogonal decomposition extensions for parametric applications in compressible aerodynamics," AIAA paper, vol. 2003-4213, pp. 1-11, 2003.

[55] A. Qamar and S. Sanghi, "Steady supersonic flow-field predictions using proper orthogonal decomposition technique," Computers \& Fluids, vol. 38, no. 6, pp. 1218-1231, 2009.

[56] D. Alonso, A. Velazquez, and J. Vega, "A method to generate computationally efficient reduced order models," Computer Methods in Applied Mechanics and Engineering, vol. 198, no. 33, pp. 2683-2691, 2009.

[57] J. Lei and S. Liu, "Temperature field reconstruction from the partial measurement data using the gappy proper orthogonal decomposition," IET Science, Measurement \& Technology, vol. 7, no. 3, pp. 171-179, 2013. 
[58] T. Bui-Thanh, M. Damodaran, and K. E. Willcox, "Aerodynamic data reconstruction and inverse design using proper orthogonal decomposition," AIAA journal, vol. 42, no. 8, pp. 1505-1516, 2004.

[59] K. Willcox, "Unsteady flow sensing and estimation via the gappy proper orthogonal decomposition," Computers $\&$ fluids, vol. 35, no. 2, pp. 208-226, 2006.

[60] L. Sirovich, "Turbulence and the dynamics of coherent structures - part I: Coherent structures," Quarterly of Applied Mathematics, vol. 45, pp. 561571, Oct. 1987.

[61] G.-B. Huang, Q.-Y. Zhu, and C.-K. Siew, "Extreme learning machine: theory and applications," Neurocomputing, vol. 70, no. 1, pp. 489-501, 2006.

[62] E. L. Lawler and D. E. Wood, "Branch-and-bound methods: A survey," Operations research, vol. 14, no. 4, pp. 699-719, 1966.

[63] W. J. Welch, "Branch-and-bound search for experimental designs based on D optimality and other criteria," Technometrics, vol. 24, no. 1, pp. 41-48, 1982.

[64] S. Joshi and S. Boyd, "Sensor selection via convex optimization," Signal Processing, IEEE Transactions on, vol. 57, no. 2, pp. 451-462, 2009.

[65] J. Ranieri, A. Chebira, and M. Vetterli, "Near-optimal sensor placement for linear inverse problems," Signal Processing, IEEE Transactions on, vol. 62, no. 5, pp. 1135-1146, 2014.

[66] S. Liu, A. Vempaty, M. Fardad, E. Masazade, and P. K. Varshney, "Energyaware sensor selection in field reconstruction," Signal Processing Letters, IEEE, vol. 21, no. 12, pp. 1476-1480, 2014.

[67] B. Yildirim, C. Chryssostomidis, and G. Karniadakis, "Efficient sensor placement for ocean measurements using low-dimensional concepts," Ocean Modelling, vol. 27, no. 3, pp. 160-173, 2009. 
[68] P. Astrid, S. Weiland, K. Willcox, and T. Backx, "Missing point estimation in models described by proper orthogonal decomposition," Automatic Control, IEEE Transactions on, vol. 53, no. 10, pp. 2237-2251, 2008.

[69] H. Jamali-Rad, A. Simonetto, and G. Leus, "Sparsity-aware sensor selection: Centralized and distributed algorithms," Signal Processing Letters, IEEE, vol. 21, no. 2, pp. 217-220, 2014.

[70] S. P. Chepuri and G. Leus, "Continuous sensor placement," Signal Processing Letters, IEEE, vol. 22, no. 5, 2015.

[71] M. Shamaiah, S. Banerjee, and H. Vikalo, "Greedy sensor selection: Leveraging submodularity," in Decision and Control (CDC), 49th IEEE Conference on, 2010, pp. 2572-2577.

[72] K. Cohen, S. Siegel, and T. McLaughlin, "A heuristic approach to effective sensor placement for modeling of a cylinder wake," Computers $\&$ fluids, vol. 35, no. 1, pp. 103-120, 2006.

[73] L. Yao, W. A. Sethares, and D. C. Kammer, "Sensor placement for on-orbit modal identification via a genetic algorithm," AIAA journal, vol. 31, no. 10, pp. 1922-1928, 1993.

[74] S. Lau, R. Eichardt, L. Di Rienzo, and J. Haueisen, "Tabu search optimization of magnetic sensor systems for magnetocardiography," Magnetics, IEEE Transactions on, vol. 44, no. 6, pp. 1442-1445, 2008.

[75] M. Naeem, S. Xue, and D. Lee, "Cross-entropy optimization for sensor selection problems," in Communications and Information Technology, 9th International Symposium on, 2009, pp. 396-401.

[76] S. P. Chepuri and G. Leus, "Sparsity-promoting sensor selection for nonlinear measurement models," Signal Processing, IEEE Transactions on, vol. 63 , no. 3, 2015. 
[77] S. P. Chepuri, G. Leus et al., "Sparsity-exploiting anchor placement for localization in sensor networks," in Signal Processing Conference, Proceedings of the 21st European, 2013, pp. 1-5.

[78] Y. Mo, R. Ambrosino, and B. Sinopoli, "Sensor selection strategies for state estimation in energy constrained wireless sensor networks," Automatica, vol. 47, no. 7, pp. 1330-1338, 2011.

[79] X. Shen and P. K. Varshney, "Sensor selection based on generalized information gain for target tracking in large sensor networks," Signal Processing, IEEE Transactions on, vol. 62, no. 2, pp. 363-375, 2014.

[80] E. Iuliano and D. Quagliarella, "Proper orthogonal decomposition, surrogate modelling and evolutionary optimization in aerodynamic design," Computers E Fluids, vol. 84, pp. 327-350, 2013.

[81] X. An and F. Pellacini, "Appprop: all-pairs appearance-space edit propagation," ACM Transactions on Graphics (TOG), vol. 27, no. 3, p. 40, 2008.

[82] L. Xu, Q. Yan, and J. Jia, "A sparse control model for image and video editing," ACM Transactions on Graphics (TOG), vol. 32, no. 6, p. 197, 2013.

[83] G. H. Golub and C. F. Van Loan, Matrix computations. JHU Press, 2013.

[84] A. P. Raghupathy, U. Ghia, K. Ghia, and W. Maltz, "Boundary-conditionindependent reduced-order modeling of complex electronic packages by podgalerkin methodology," Components and Packaging Technologies, IEEE Transactions on, vol. 33, no. 3, pp. 588-596, 2010.

[85] H. Zhou, Y. C. Soh, C. Jiang, and X. Wu, "Compressed representation learning for fluid field reconstruction from sparse sensor observations," in Neural Networks (IJCNN), International Joint Conference on, 2015.

[86] J. Meinguet, "Multivariate interpolation at arbitrary points made simple," Journal of Applied Mathematics and Physics, vol. 30, no. 2, pp. 292-304, 1979. 
[87] W. F. Schmidt, M. A. Kraaijveld, and R. P. Duin, "Feedforward neural networks with random weights," in Pattern Recognition, 11th International Conference on , 1992, pp. 1-4.

[88] Y. H. Pao and Y. Takefji, "Functional-link net computing," IEEE Computer Journal, vol. 25, no. 5, pp. 76-79, 1992.

[89] Y.-H. Pao, G.-H. Park, and D. J. Sobajic, "Learning and generalization characteristics of the random vector functional-link net," Neurocomputing, vol. 6, no. 2, pp. 163-180, 1994.

[90] B. Igelnik and Y.-H. Pao, "Stochastic choice of basis functions in adaptive function approximation and the functional-link net," Neural Networks, IEEE Transactions on, vol. 6, no. 6, pp. 1320-1329, 1995.

[91] G.-B. Huang, Q.-Y. Zhu, and C.-K. Siew, "Extreme learning machine: a new learning scheme of feedforward neural networks," in Neural Networks (IJCNN), International Joint Conference on, 2004.

[92] G.-B. Huang, "An insight into extreme learning machines: random neurons, random features and kernels," Cognitive Computation, vol. 6, no. 3, pp. 376$390,2014$.

[93] G. Huang, G.-B. Huang, S. Song, and K. You, "Trends in extreme learning machines: a review," Neural Networks, vol. 61, pp. 32-48, 2015.

[94] G.-B. Huang, H. Zhou, X. Ding, and R. Zhang, "Extreme learning machine for regression and multiclass classification," Systems, Man, and Cybernetics, Part B: Cybernetics, IEEE Transactions on, vol. 42, no. 2, pp. 513-529, 2012.

[95] G.-B. Huang, L. Chen, and C.-K. Siew, "Universal approximation using incremental constructive feedforward networks with random hidden nodes," Neural Networks, IEEE Transactions on, vol. 17, no. 4, pp. 879-892, 2006.

[96] G.-B. Huang and L. Chen, "Convex incremental extreme learning machine," Neurocomputing, vol. 70, no. 16, pp. 3056-3062, 2007. 
[97] — - "Enhanced random search based incremental extreme learning machine," Neurocomputing, vol. 71, no. 16, pp. 3460-3468, 2008.

[98] K. P. Murphy, "Machine learning: a probabilistic perspective," 2012.

[99] C. H. Papadimitriou and K. Steiglitz, Combinatorial optimization: algorithm$s$ and complexity. Courier Corporation, 1998.

[100] S. Boyd and L. Vandenberghe, Convex optimization. Cambridge university press, 2004.

[101] M. Grant, S. Boyd, and Y. Ye, "CVX: Matlab software for disciplined convex programming," 2008.

[102] J. J. Benedetto and M. Fickus, "Finite normalized tight frames," Advances in Computational Mathematics, vol. 18, no. 2-4, pp. 357-385, 2003.

[103] J. Kovacevic and A. Chebira, "Life beyond bases: The advent of frames (part i)," Signal Processing Magazine, IEEE, vol. 24, no. 4, pp. 86-104, 2007.

[104] X. Shen, S. Liu, and P. Varshney, "Sensor selection for nonlinear systems in large sensor networks," Aerospace and Electronic Systems, IEEE Transactions on, vol. 50, no. 4, pp. 2664-2678, 2014.

[105] S. Liu, M. Fardad, E. Masazade, and P. K. Varshney, "Optimal periodic sensor scheduling in networks of dynamical systems," Signal Processing, IEEE Transactions on, vol. 62, no. 12, pp. 3055-3068, 2014.

[106] V. Roy, A. Simonetto, and G. Leus, "Spatio-temporal sensor management for environmental field estimation," Signal Processing, vol. 128, pp. 369-381, 2016.

[107] S. P. Chepuri and G. Leus, "Sparsity-promoting adaptive sensor selection for non-linear filtering," in Acoustics, Speech and Signal Processing (ICASSP), IEEE International Conference on, 2014, pp. 5080-5084. 
[108] S. Liu, E. Masazade, M. Fardad, and P. K. Varshney, "Sparsity-aware field estimation via ordinary kriging," in Acoustics, Speech and Signal Processing (ICASSP), IEEE International Conference on, 2014.

[109] H. Wang, K. Yao, G. Pottie, and D. Estrin, "Entropy-based sensor selection heuristic for target localization," in Information processing in sensor networks, 3rd International Symposium on, 2004.

[110] D. MacKay, "Information-based objective functions for active data selection," Neural computation, vol. 4, no. 4, pp. 590-604, 1992.

[111] C. Guestrin, A. Krause, and A. P. Singh, "Near-optimal sensor placements in gaussian processes," in Machine learning, 22nd International Conference on , 2005 .

[112] R. Thompson, "The behavior of eigenvalues and singular values under perturbations of restricted rank," Linear Algebra and its Applications, vol. 13, no. 1, pp. 69-78, 1976.

[113] K.-C. Toh, M. J. Todd, and R. H. Tütüncü, "SDPT3: a matlab software package for semidefinite programming, version 1.3," Optimization methods and software, vol. 11, no. 1-4, pp. 545-581, 1999.

[114] A. J. Miller and N.-K. Nguyen, "Algorithm as 295: A fedorov exchange algorithm for D-optimal design," Applied Statistics, pp. 669-677, 1994.

[115] H. P. Wynn, "Results in the theory and construction of D-optimum experimental designs," Journal of the Royal Statistical Society. Series B (Methodological), pp. 133-147, 1972. 